Supporting Information for

\title{
Copolymerization of Ethylene with Polar Monomers by Anionic Substitution. A Theoretical Study Based on Acrylonitrile and the Brookhart Diimine Catalyst
}

Miklos J. Szabo, ${ }^{\text {a }}$ Natasha M. Galea, ${ }^{\mathrm{a}}$ Artur Michalak, ${ }^{\mathrm{b}}$ Sheng-Yong Yang, ${ }^{\mathrm{a}}$ Laurent F. Groux, ${ }^{\mathrm{a}}$ Warren E. Piers ${ }^{\mathrm{a}}$ and Tom Ziegler ${ }^{\mathrm{a}, *}$

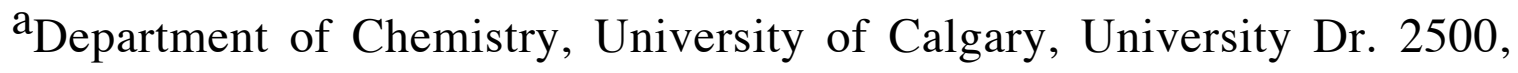
Calgary, AB, Canada T2N 1N4 ${ }^{b}$ Department of Theoretical Chemistry, Faculty of Chemistry, Jagiellonian University, R. Ingardena 3, 30-060 Cracow, Poland ziegler@ucalgary.ca

Content: The most stable conformers of the crucial compounds of 16, 17, 19, 20, 21, 22, 23, 24, 25, 26, 27, 28 are shown (the atomic symbols followed by three Cartesian Coordinates, in $\AA$ ). The structure labels correspond to those of Tables 3-4 and Schemes $5-6$. 

69

$16 \mathrm{R}=\mathrm{Me}$

Ni $0.001164 \quad 0.003376 \quad 0.014577$ $\begin{array}{lllll}\mathrm{N} & 1.839901 & 0.003869 & 0.044828\end{array}$ $\begin{array}{lllll}\text { C } 2.674511 & 1.176151 & 0.032261\end{array}$ C $2.409206-1.186452-0.046486$ C $3.881445-1.418340-0.127376$ H $4.436445-0.482552-0.264776$ H $4.123464-2.103231-0.954592$ H $4.240608-1.9017860 .798419$ $\begin{array}{llll}\text { C } 1.480963 & -2.310968 & -0.005114\end{array}$ $\begin{array}{llll}\text { C } & 1.480963 & -2.310968 & -0.005114 \\ \text { C } & 1.961609 & -3.706809 & -0.226347\end{array}$ H $2.542912-3.748171-1.161776$ $\begin{array}{llll}\text { H } & 2.542912 & -3.748171 & -1.161776 \\ \text { H } 2.622465 & -4.054805 & 0.583216\end{array}$ $\begin{array}{llll}\mathrm{H} & 2.622465 & -4.054805 & 0.583216 \\ \mathrm{H} & 1.115252 & -4.398849 & -0.314790\end{array}$ $\begin{array}{lllll}\mathrm{H} & 1.115252 & -4.398849 & -0.314790 \\ \mathrm{~N} & 0.236183 & -1.945674 & 0.169347\end{array}$ $\begin{array}{llll}\mathrm{N} & 0.236183 & -1.945674 & 0.169347 \\ \mathrm{C} & -0.836603 & -2.857639 & 0.339008\end{array}$ $\begin{array}{llll}\text { C }-0.836603 & -2.857639 & 0.339008 \\ \text { C }-1.956811 & -2.640349 & -0.492615\end{array}$ $\begin{array}{llll}\text { C } & -1.956811 & -2.640349 & -0.492615 \\ \text { C }-0.827925 & -3.858759 & 1.339973\end{array}$ C $-3.013069-3.547988-0.411931$ $\begin{array}{llll}\text { C }-1.919741 & -4.730993 & 1.378427\end{array}$ $\begin{array}{llll}\text { C }-2.985907 & -4.607571 & 0.492400\end{array}$ B $-2.068061-1.331481-1.424050$ F $-1.049880-1.180618-2.371551$ F $-3.330117-1.131777-1.949507$ F $-1.848573-0.142240-0.411889$ $\begin{array}{llll}\mathrm{F} & -1.848573 & -0.142240 & -0.411889\end{array}$ $\begin{array}{llll}\text { C } & 0.197344 & -3.914077 & 2.461784 \\ \text { C } & 0.723744 & -5.328324 & 2.737181\end{array}$ $\begin{array}{llll}\text { C } & 0.723744 & -5.328324 & 2.737181 \\ \text { C } & -0.409932 & -3.305174 & 3.738582\end{array}$ $\begin{array}{llll}\text { H } 1.053549 & -3.281339 & 2.190906\end{array}$ H $1.120461-5.805287 \quad 1.828847$ H $1.524615 \quad-5.294070 \quad 3.492068$ H $-0.070691 \quad-5.977482 \quad 3.135507$ H $-0.763476-2.279796 \quad 3.554642$ н $-1.270439-3.901886 \quad 4.079706$ H $0.337359-3.2829214 .547753$ H $-3.878442-3.395665-1.058212$ H $-3.815380-5.3143630 .540755$ $\begin{array}{llll}\mathrm{H} & -1.946167 & -5.514617 & 2.136285\end{array}$ C $2.8635921 .859088-1.182811$ C $3.2323681 .625417 \quad 1.240111$ C $3.675136 \quad 2.996722-1.165118$ $\begin{array}{llll}\text { C } & 3.675136 & 2.996722 & -1.165118 \\ \text { C } & 4.027671 & 2.774102 & 1.207797\end{array}$ $\begin{array}{llll}\text { C } 4.027671 & 2.774102 & 1.207797\end{array}$ $\begin{array}{llll}\text { C } 2.212594 & 1.379737 & -2.467969\end{array}$ $\begin{array}{llll}\text { C } & 2.212594 & 1.379737 & -2.467969 \\ \text { C } 3.152628 & 0.466476 & -3.267679\end{array}$ $\begin{array}{llll}\text { C } 1.721596 & 2.530445 & -3.352205\end{array}$ H $1.329727 \quad 0.782662 \quad-2.187518$ H $4.064148 \quad 1.012040-3.562244$ H $2.648176 \quad 0.112572 \quad-4.179896$ H $3.458783-0.415303-2.687099$ H $1.1021023 .238555-2.781288$ $\begin{array}{lllll}\text { H } & 1.113397 & 2.125778 & -4.175938\end{array}$ H $2.560229 \quad 3.085643 \quad-3.802949$ $\begin{array}{llll}\text { C } 2.942230 & 0.938742 & 2.564155\end{array}$ $\begin{array}{llll}\text { C } 1.857778 & 1.708523 & 3.335977\end{array}$ C $4.198623 \quad 0.752868 \quad 3.423808$ H $2.536722 \quad-0.060703 \quad 2.347886$ H $0.9324901 .786647 \quad 2.747175$ H $2.201850 \quad 2.728952 \quad 3.569486$ H $1.625784 \quad 1.196607 \quad 4.282995$ H 5.0022280 .2507802 .863870 H $4.588373 \quad 1.7172443 .783897$ $\begin{array}{llll}\text { H } & 3.959687 & 0.146976 & 4.311195 \\ \text { H } & 3.843605 & 3.548743 & -2.092288\end{array}$ H $3.843605 \quad 3.548743 \quad-2.092288$ $\begin{array}{lllll}\text { H } & 4.886503 & 4.346824 & 0.007235\end{array}$ H $4.471386 \quad 3.1453192 .133786$ C $-0.265677 \quad 1.902023 \quad 0.017130$ H $-1.089697 \quad 2.035972 \quad 0.739259$ H $-0.620324 \quad 2.159641 \quad-0.994114$ $\begin{array}{lllll}\text { H } & 0.617800 & 2.489650 & 0.296012\end{array}$ 75

$16 \mathrm{R}=\mathrm{Me} \mathrm{ET} / \mathrm{pi}$
$\mathrm{Ni} 0.015001-0.011594-0.006078$ N $2.002894-0.0055020 .015600$ $\begin{array}{llll}\mathrm{N} & 2.002894 & -0.005502 & 0.015600\end{array}$ C $2.558739-1.186480-0.017462$ $\begin{array}{llll}\text { C } 2.558739 & -1.186480 & -0.017462 \\ \text { C } 4.026420 & -1.454667 & -0.015394\end{array}$ $\begin{array}{llll}\text { C } & 4.026420 & -1.454667 & -0.015394 \\ \mathrm{H} & 4.608249 & -0.538834 & 0.143051\end{array}$ $\begin{array}{llll}\mathrm{H} & 4.608249 & -0.538834 & 0.143051 \\ \mathrm{H} & 4.324374 & -1.896414 & -0.981047\end{array}$ $\begin{array}{llll}\text { H } & 4.324374 & -1.896414 & -0.981047 \\ \text { H } & 4.283048 & -2.190678 & 0.763358\end{array}$ $\begin{array}{llll}\text { H } & 4.283048 & -2.190678 & 0.763358 \\ \text { C } & 1.611659 & -2.308320 & -0.153359\end{array}$ $\begin{array}{llll}\text { C } & 1.611659 & -2.308320 & -0.153359 \\ \text { C } & 2.108790 & -3.669122 & -0.501363\end{array}$ $\begin{array}{llll}\text { C } & 2.108790 & -3.669122 & -0.501363 \\ \text { H } & 2.640791 & -3.624845 & -1.465261\end{array}$ $\begin{array}{lllll}\text { H } 2.812842 & -4.037004 & 0.261556\end{array}$ H $1.277160-4.375768-0.606349$ $\begin{array}{lllll}\text { N } 0.363064 & -1.974139 & -0.042937\end{array}$ C $-0.666115-2.873200-0.485010$ C $-1.020705-2.713060-1.838409$ $\begin{array}{llll}\text { C }-1.304107 & -3.765081 & 0.386897\end{array}$ $\begin{array}{llll}\text { C } & -2.053331 & -3.522475 & -2.317315\end{array}$ $\begin{array}{llll}\text { C } & -2.053331 & -3.522475 & -2.317315 \\ \text { C } & -2.329555 & -4.553381 & -0.152644\end{array}$ C $-2.701134-4.439403-1.488200$ B $-0.270531-1.623841-2.782348$ F $1.118559-1.903410-2.916472$ F $-0.879342-1.488976-4.027482$
F $-0.346855-0.285440-2.107189$ C $-0.919691-3.9000091 .849960$ C $-0.363075-5.2970542 .16462$ $\begin{array}{llll}\text { C }-2.098281 & -3.573873 & 2.779471\end{array}$ H $-0.119300-3.1698362 .051709$ H $0.501761-5.540972 \quad 1.530153$ H $-1.129894 \quad-6.069110 \quad 1.995191$ H $-0.049701-5.358363 \quad 3.218735$ H $-2.914445-4.301175 \quad 2.646128$ H $-2.506442-2.572592 \quad 2.578429$ $\begin{array}{llll}H & -2.506442 & -2.572592 & 2.578429\end{array}$ H $-1.779931-3.6169403 .833040$ $\begin{array}{llll}\mathrm{H} & -2.347752 & -3.417958 & -3.363075\end{array}$ H $-3.503469-5.065235-1.881712$ $\begin{array}{llll}\mathrm{H} & -2.846459 & -5.269169 & 0.488524 \\ \mathrm{C} & 3.289148 & 1.666879 & -1.220927\end{array}$ $\begin{array}{llll}C & 3.289148 & 1.666879 & -1.220927\end{array}$ C $3.1565291 .784182 \quad 1.236372$ C $4.1009492 .805658-1.202346$ $\begin{array}{llll}\text { C } 3.980045 & 2.920133 \quad 1.199247\end{array}$ C $4.456107 \quad 3.426076-0.007500$ $\begin{array}{llll}\text { C } 2.896119 & 1.043254 & -2.547113\end{array}$ $\begin{array}{llll}\text { C } 2.058762 & 2.025283 & -3.398544\end{array}$ $\begin{array}{llll}\text { C } 4.115850 & 0.574470 & -3.353221\end{array}$ H $2.261164 \quad 0.168042 \quad-2.360767$ H $1.1780792 .379906 \quad-2.844079$ н $2.6588192 .900954 \quad-3.698648$ $1.707268-1.513379-4.307858$ H $4.756553-0.105965-2.768870$ H $4.756553-0.105965-2.768870$ $\begin{array}{llll}\text { H } & 4.737620 & 1.427832 & -3.674222\end{array}$ 3.7482900 .0385180 .4 .255033 $\begin{array}{llll}C & 2.644188 & 1.2501342 .559806\end{array}$

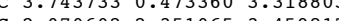
$\begin{array}{llll}C & 2.070602 & 2.351065 & 3.459817\end{array}$ H 4.5878601 .1409593 .561103 H $4.137318 \quad-0.363571 \quad 2.722231$ H $3.342857 \quad 0.068290 \quad 4.262378$ н $2.858127 \quad 3.035582 \quad 3.816782$ H $1.3148172 .950312 \quad 2.927072$ $\begin{array}{llll}\text { H } 1.597710 & 1.901814 & 4.348881\end{array}$ H $4.458337 \quad 3.216951-2.149357$ H $5.094973 \quad 4.312624 \quad-0.016759$ H $4.245465 \quad 3.4199602 .133756$ C $-0.195903 \quad 1.906310-0.212268$ H $-0.5310771 .956056-1.260513$ $\begin{array}{lllll}\text { H } & 0.780127 & 2.390808 & -0.072469\end{array}$ $\begin{array}{llll}\text { H } & -0.946627 & 2.353932 & 0.453186\end{array}$ $\begin{array}{llll}\text { C } & -0.942169 & 0.049755 & 1.731784\end{array}$ $\begin{array}{llll}\text { C }-1.874564 & -0.111396 & 0.668578\end{array}$ $\begin{array}{llll}C & -1.874564 & -0.111396 & 0.668578\end{array}$ $\begin{array}{llll}\mathrm{H}-0.851988 & 1.018753 & 2.221794 \\ \mathrm{H}-0.654214 & -0.814820 & 2.321294\end{array}$ $\begin{array}{llll}\text { H } & -0.654214 & -0.814820 & 2.32129 \\ \text { H } & -2.458347 & 0.735849 & 0.302131\end{array}$ H $-2.264010-1.0991010 .40470$ H 1.8290420 .5457392 .335585 76

$16 \mathrm{R}=\mathrm{Me} \mathrm{AC} / \mathrm{pi}$

Ni $0.009163-0.004420-0.009692$ N $2.018998-0.007775-0.021706$ C $2.8478201 .164146-0.005589$ $\begin{array}{llll}\text { C } 2.558175 & -1.190768 & 0.046724\end{array}$

$\begin{array}{llll}\text { C } 4.018146 & -1.470540 & 0.161159\end{array}$ $\begin{array}{llll}C & 4.018146 & -1.470540 & 0.161159\end{array}$ $\begin{array}{lllll}\mathrm{H} & 4.613652 & -0.552074 & 0.099334\end{array}$ H $4.337723-2.161230-0.634123$ H $4.228123-1.9713031 .121828$ C $1.605753-2.317259-0.065763$ C $2.112228-3.683438-0.376036$ H $2.663986-3.654669-1.329651$ H $2.800434-4.0340350 .409056$ H $1.285104-4.393965-0.485172$ N $0.356766 \quad-1.987057 \quad 0.018964$ C $-0.664261-2.909296-0.406548$ $\begin{array}{llll}\text { C } & -1.041922 & -2.743988 & -1.753742\end{array}$ $\begin{array}{llll}\text { C }-1.251285 & -3.845004 & 0.457693\end{array}$ $\begin{array}{llll}\text { C }-2.065440 & -3.566053 & -2.229592\end{array}$ $\begin{array}{llll}\text { C }-2.274129 & -4.639206 & -0.079478\end{array}$ C $-2.682606-4.503796-1.401721$ B $-0.290989-1.663730-2.697560$ B $-0.290989-1.663730-2.697560$ F $1.098009-1.877124-2.773185$ $\begin{array}{llll}\mathrm{F}-0.825398 & -1.591759 & -3.993305\end{array}$ F $-0.457172-0.301167-2.052368$ C $-0.815487-4.0392591 .899602$ $C-0.254834-5.4509642 .137989$
$C$ $\begin{array}{llll}C & -1.960062 & -3.756216 & 2.883798\end{array}$ $\begin{array}{llll}\mathrm{H} & -0.012740 & -3.319715 & 2.116577\end{array}$ н $0.574042 \quad-5.683940 \quad 1.453190$ H $0.114722-5.540718 \quad 3.171361$ H $-1.034018-6.215290 \quad 1.990642$ H $-2.368253-2.743648 \quad 2.751756$ H $-1.597655 \quad-3.840645 \quad 3.919487$ H $-2.783436-4.4749242 .746518$ H $-2.372188-3.459538-3.271421$ H $-3.483031-5.136448-1.788051$ H $-2.755989-5.3831850 .556696$ C $3.079997 \quad 1.813236-1.235462$

$\begin{array}{lll}\text { C } 3.079997 & 1.813236 & -1.235462 \\ \text { C } 3.335975 & 1.675685 & 1.210299\end{array}$

$\begin{array}{llll}\text { C } 3.335975 & 1.675685 & 1.210299 \\ \text { C } 3.814799 & 3.001146 & -1.220260\end{array}$ C 4.0631232 .8724281 .170546 $\begin{array}{llll}\text { C } 4.301419 & 3.535275 & -0.028859\end{array}$ C $2.5980901 .219767-2.544267$

$\begin{array}{llll}\text { C } 2.114399 & 2.272582 & -3.546400\end{array}$ C $3.707627 \quad 0.349266-3.197182$ H $1.755744 \quad 0.547573 \quad-2.333495$ $\begin{array}{lllll}\mathrm{H} & 1.394666 & 2.970938 & -3.088173\end{array}$ H $1.6157841 .770485-4.391286$ H $2.9506412 .862057-3.959350$ H $4.039035-0.454358-2.521410$ H $3.317635-0.120782-4.114592$ H $4.584574 \quad 0.969897 \quad-3.452102$ C $3.092538 \quad 1.002493 \quad 2.550460$ C 2.2440341 .8959333 .480886 C $2.2440341 .895933 \quad 3.480886$ $\begin{array}{llll}C & 4.409741 & 0.609726 & 3.245718\end{array}$ H 2.5191460 .0799332 .383173 H 1.3197602 .2271452 .981334 H $2.804463 \quad 2.798859 \quad 3.778485$ H $1.970457 \quad 1.339813 \quad 4.392567$ H $5.040149-0.0242112 .601452$ H $4.998977 \quad 1.503207 \quad 3.513853$ H 4.1937230 .0558454 .174852 H $4.006203 \quad 3.521452-2.160880$ H $4.868374 \quad 4.467771-0.037242$ H $4.4481803 .291431 \quad 2.103144$ C $-0.165115 \quad 1.908418 \quad-0.312613$ H $0.654297 \quad 2.427473 \quad 0.203407$ H $-0.060319 \quad 1.947280-1.406187$ н $-1.143654 \quad 2.299589-0.002853$ C $-1.774916 \quad-0.122493 \quad 0.849175$ $\begin{array}{lllll}C & -1.774916 & -0.122493 & 0.849175\end{array}$ C $-0.7963250 .297355 \quad 1.788016$ H $-2.115401-1.1605910 .816107$ $\begin{array}{lllll}\mathrm{H} & -2.466924 & 0.620286 & 0.449121\end{array}$ $\begin{array}{llll}\text { H } & -0.754746 & 1.352465 & 2.073274 \\ \text { C } & -0.199352 & -0.562047 & 2.756900\end{array}$ $\begin{array}{llll}\text { C } & -0.199352 & -0.562047 & 2.756900 \\ \mathrm{~N} & 0.328157 & -1.217548 & 3.568328\end{array}$ 76 $16 \mathrm{R}=\mathrm{Me} \mathrm{AC} / \mathrm{N}-\mathrm{t}$ Ni $0.144773-0.1804290 .546824$ N $1.947757-0.020897-0.038569$ $\begin{array}{llll}\text { C } 2.656609 & 1.218688 & -0.203699\end{array}$ $\begin{array}{llll}\text { C } 2.523139 & -1.141981 & -0.404193\end{array}$ C $3.910791-1.262009-0.930178$ H $4.475851-0.329329-0.813402$ H $3.876015-1.518971-2.002868$ H $4.444528-2.081775-0.4238$ C $1.652158-2.081775-0.423939$ C $1.652158-2.322636-0.33585$ $\begin{array}{llll}C & 2.088793 & -3.630573-0.896934\end{array}$ H $2.311078-3.516012-1.969131$ H $2.999657-3.985806-0.388503$ H $1.296083-4.381567-0.796220$ $\begin{array}{llll}\text { N } 0.489459 & -2.071952 & 0.186690\end{array}$ $\begin{array}{llll}\text { C } & -0.596545 & -2.997069 & 0.030043\end{array}$ C $-1.294160-2.889430-1.188391$ C $-0.964499-3.859832 \quad 1.071228$ $\begin{array}{llll}\text { C }-2.391458 & -3.741917 & -1.350369\end{array}$ C $-2.067233-4.693777 \quad 0.846034$ $\begin{array}{llll}\text { C }-2.772756 & -4.641767 & -0.353936\end{array}$ В $-0.890004-1.783559-2.328940$ F $-0.918728-0.462160-1.727507$ F $0.449475-1.995992-2.803364$ F $-1.791508-1.824695-3.403024$ $\begin{array}{llll}F & -1.791508 & -1.824695 & -3.403024\end{array}$ C $-0.183768-3.9300052 .371551$ $\begin{array}{llll}\text { C } 0.639705 & -5.224650 & 2.456743\end{array}$

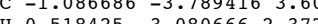
$\begin{array}{llll}\text { H } 0.518425 & -3.080666 & 2.377712\end{array}$ $\begin{array}{llll}\mathrm{H} & 1.327363 & -5.321886 & 1.603735\end{array}$ $\begin{array}{cccc}\text { H } & 1.232209 & -5.247185 & 3.385215 \\ \mathrm{H} & -0.023225 & -6.104839 & 2.452609\end{array}$ $\begin{array}{llll}\mathrm{H} & -0.023225 & -6.104839 & 2.452609 \\ \mathrm{H} & -1.672468 & -2.859309 & 3.564685\end{array}$ H $-1.672468-2.8593093 .564685$ H $-0.479055-3.777537 \quad 4.521997$ H $-1.790337-4.632822 \quad 3.681844$ H $-2.948722-3.682497-2.287279$ H $-3.623080-5.308151-0.511197$ Н $-2.373293 \quad-5.399384 \quad 1.620407$ C $2.729244 \quad 1.795327-1.487108$ C $3.1729861 .857722 \quad 0.938301$ $\begin{array}{llll}\text { C } 3.358400 & 3.037962 & -1.599394\end{array}$ C 3.7980253 .0965760 .772336 C $3.8941913 .692064-0.482276$ $\begin{array}{llll}\text { C } & 3.894191 & 3.692064 & -0.482276 \\ \text { C } 2.116135 & 1.154903 & -2.721285\end{array}$ $\begin{array}{llll}\text { C } & 0.116135 & 1.154903 & -2.721285 \\ \text { C } 3.14299 & 1.953308 & -3.204522\end{array}$ $\begin{array}{llll}C & 0.895799 & 1.953308 & -3.204522 \\ C & .142907 & 1.002489 & -3.853998\end{array}$ $\begin{array}{lllll}\text { C } & 3.142907 & 1.002489 & -3.853998 \\ \mathrm{H} & 1.742931 & 0.153701 & -2.467358\end{array}$ $\begin{array}{lllll}\mathrm{H} & 1.742931 & 0.153701 & -2.467358 \\ \mathrm{H} & 0.128123 & 2.011978 & -2.421087\end{array}$ $\begin{array}{llll}\mathrm{H} & 0.128123 & 2.011978 & -2.421087 \\ \mathrm{H} & 1.184028 & 2.975146 & -3.500727\end{array}$ $\begin{array}{llll}\text { H } & 1.184028 & 2.975146 & -3.500727 \\ \text { H } & 0.446110 & 1.448005 & -4.071836\end{array}$ H $4.052885 \quad 0.482132-3.516754$ H $3.4483851 .982406-4.255247$ $\begin{array}{lllll}\text { H } & 2.698305 & 0.425184 & -4.678904\end{array}$ C $3.059183 \quad 1.210196 \quad 2.305909$ $\begin{array}{llll}\text { C } 2.908827 & 2.223135 & 3.444554\end{array}$ $\begin{array}{llll}\text { C } 4.249517 & 0.275433 & 2.574533\end{array}$ H $2.1441890 .593135 \quad 2.289785$ H $4.323957 \quad-0.516344 \quad 1.815178$ 
H $3.420240 \quad 3.514472 \quad-2.578945$ H $4.375127 \quad 4.667020-0.595618$ H $4.202655 \quad 3.615047 \quad 1.643317$ C $-0.200361 \quad 1.726394 \quad 0.738125$ $\begin{array}{lllll}\text { H } & 0.617581 & 2.307521 & 0.295133\end{array}$ H $-1.143204 \quad 1.889030 \quad 0.193398$ H $-0.328836 \quad 1.968097 \quad 1.804013$ N $-1.417062-0.436561 \quad 1.396114$ $\begin{array}{llll}\text { C }-2.481742 & -0.627108 & 1.828104\end{array}$ $\begin{array}{llll}\text { C }-3.765653 & -0.849463 & 2.362385 \\ \text { H }-4.112489 & -0.128716 & 3.107032\end{array}$ $\begin{array}{lll}\mathrm{C}-4.112489 & -0.128716 & 3.107032\end{array}$ $\begin{array}{lll}\mathrm{C}-4.530961-1.884932 & 1.966634\end{array}$ $\mathrm{H}-4.178143-2.5940291 .212420$ 952.395029

$6 \mathrm{R}=$ CHCNEt $\mathrm{ET} / \mathrm{p}$

Ni $0.009385-0.004132-0.010056$ $\mathrm{N} 2.034215-0.003810-0.009605$ C $2.880881 \quad 1.155676 \quad 0.002068$ $\begin{array}{llll}\text { C } 2.571894 & -1.189040 & 0.067977\end{array}$ C $4.030236-1.4867010 .168480$ H $4.636123-0.5738540 .130808$ H $4.335560-2.155885-0.651168$ H $4.243415-2.0220341 .109026$ C $1.608884-2.3012740 .000040$ $\begin{array}{llll}\text { C } 2.100016 & -3.718118 & -0.276013\end{array}$ $\begin{array}{llll}\text { C } & 2.100016 & -3.718118 & -0.276013 \\ \mathrm{H} & 2.595971 & -3.729116 & -1.261928\end{array}$ $\begin{array}{llll}\text { H } 2.826490 & -4.032826 & 0.491231\end{array}$ $\begin{array}{llll}H & 1.256011 & -4.419984 & -0.307562\end{array}$ N $-0.680821-2.876139-0.230024$ C $-0.680821-2.876139-0.230024$ C $-1.074241-2.834680-1.578560$ $\begin{array}{llll}C & -1.294793 & -3.679524 & 0.742561\end{array}$ $\begin{array}{llll}\text { C }-2.139792 & -3.662348 & -1.949906\end{array}$ $\begin{array}{llll}\text { C }-2.349818 & -4.494438 & 0.316636\end{array}$ C $-2.779403-4.488922-1.027167$ B $-0.323625-1.856473-2.634685$ F $1.054460-2.154829-2.762025$ F $-0.951625-1.816418-3.878408$ F $-0.376068-0.451792-2.070408$ C $-0.849810-3.695803 \quad 2.193408$ $\begin{array}{llll}C & -0.225997 & -5.080510 & 2.587270\end{array}$ C $-1.987119-3.3377883 .158070$ C $-0.054455-2$. $\begin{array}{llll}\mathrm{H} & -0.054455 & -2.943673 & 2.310749\end{array}$ $\begin{array}{lllll}\text { H } & 0.137923 & -5.048975 & 3.628436\end{array}$ H $-0.984292-5.8778332 .510500$ $\begin{array}{llll}\mathrm{H}-2.425524 & -2.357749 & 2.919274\end{array}$ H $-1.614261-3.307718 \quad 4.193820$ H $-2.793134-4.086808 \quad 3.114202$ H $-2.466144-3.645360-2.994016$ H $-3.611391-5.129511-1.333920$ H $-2.853508 \quad-5.145325 \quad 1.035202$ $\begin{array}{llll}\text { C } 3.217268 & 1.734593 & -1.239146\end{array}$ $\begin{array}{llll}\text { C } 3.311168 & 1.706489 & 1.224448\end{array}$ C $4.006575 \quad 2.888047 \quad-1.230500$ $\begin{array}{lllll}\text { C } 4.097813 & 2.863532 & 1.175545\end{array}$ $\begin{array}{llll}\text { c } 4.445618 & 3.453698 & -0.035696\end{array}$ C 2.784060 $\begin{array}{llll}\text { C } 2.784060 & 1.107939 & -2.554280\end{array}$ $\begin{array}{llll}\text { C } 2.368308 & 2.144618 & -3.603180\end{array}$ $\begin{array}{llll}\text { C } 3.896889 & 0.217346 & -3.144435\end{array}$ $\begin{array}{llll}\text { H } & 1.917920 & 0.456844 & -2.363273 \\ \text { H } & 4.193231 & -0.582581 & -2.449340\end{array}$ H $4.193231-0.582581-2.449340$ H $4.793548 \quad 0.819979-3.373394$ H $3.540848-0.259450-4.072429$ H $1.658393 \quad 2.881634-3.195094$ H $3.2387242 .695907 \quad-3.997814$ H $1.887168 \quad 1.634388-4.453438$ $\begin{array}{llll}\text { C } 2.974829 & 1.095524 & 2.574145\end{array}$ $\begin{array}{llll}\text { C } 2.313609 & 2.108104 & 3.526093\end{array}$ $\begin{array}{llll}\text { C } 4.247351 & 0.499446 & 3.258899\end{array}$ H $2.262486 \quad 0.273093 \quad 2.412114$ H $4.752220 \quad-0.243907 \quad 2.622595$ H $3.969340 \quad 0.017834 \quad 4.211909$ H $4.970963 \quad 1.3022013 .480028$ H $1.4632662 .619902 \quad 3.050426$ H. 1.4632662 .6199023 .050426 H 1.9631861 .5917350 .436130 . H $4.2793363 .355396-2.178764$ $\begin{array}{lllll}\mathrm{H} & 5.054554 & 4.361667 & -0.04935 \\ \mathrm{H} & 4.437517 & 3.314479 & 2.110269\end{array}$ $\begin{array}{lllll}\text { H } & 4.437517 & 3.314479 & 2.110269 \\ \text { C } & -0.245485 & 1.918497 & -0.519102\end{array}$ $\begin{array}{lllll}\text { C } & -0.245485 & 1.918497 & -0.519102 \\ \text { H } & 0.640576 & 2.002786 & -1.168119\end{array}$ $\begin{array}{llll}\text { H } & 0.640576 & 2.002786 & -1.168119 \\ \text { C } & -0.112604 & 2.819966 & 0.584718\end{array}$ $\begin{array}{llll}\text { C } & -0.112604 & 2.819966 & 0.584718 \\ \text { N } & -0.085660 & 3.546184 & 1.502593\end{array}$ $\begin{array}{llll}\mathrm{N}-0.085660 & 3.546184 & 1.502593 \\ \mathrm{C}-1.528726 & 2.179497 & -1.319314\end{array}$ $\begin{array}{llll}\text { C }-1.409558 & 3.458725 & -2.210843\end{array}$ H $-2.3774492 .316668-0.634304$ $\mathrm{H}-1.735126 \quad 1.313783-1.962498$ н $-0.624996 \quad 3.327887 \quad-2.972702$ $\mathrm{H}-1.167083 \quad 4.343536 \quad-1.599399$ H $-2.3657763 .638690-2.729576$ $\mathrm{C}-2.3657763 .638690-2.729576$ C $-1.7586230 .124524 \quad 1.848741$ C $-1.789933-0.0748400 .920874$ $\mathrm{H}-0.350964-0.7177942 .414182$ $\begin{array}{llll}\mathrm{H} & -0.580488 & 1.113585 & 2.277961 \\ \mathrm{H} & -2.435334 & 0.757250 & 0.639541\end{array}$ $\begin{array}{llll}\text { H } & -2.197609 & -1.070621 & 0.724198\end{array}$ 83 $16 \mathrm{R}=\mathrm{CHCNEt} \mathrm{AC} / \mathrm{pi}$ Ni $0.012566-0.049504-0.070108$ $\begin{array}{llll}\mathrm{N} & 2.043843 & -0.028050 & 0.000548\end{array}$ $\begin{array}{llll}\text { C } 2.874619 & 1.145055 & 0.021438\end{array}$ C $2.567901-1.210531 \quad 0.146849$ $\begin{array}{llll}\text { C } 4.011812-1.494084 & 0.383143\end{array}$ H $4.618595-0.5845070 .303839$ $\begin{array}{llll}\mathrm{H} & 4.382462 & -2.240156 & -0.335553\end{array}$ $\begin{array}{llll}\text { H } & 4.143572 & -1.925072 & 1.390506\end{array}$ $\begin{array}{llll}\text { C } 1.622245 & -2.338699 & -0.009800\end{array}$ C $2.144775-3.710977-0.25756$ H $2.778422-3.698950-1.159353$ H $2.761135-4.0514390 .589265$ $\begin{array}{llll}\text { H } 1.326413 & -4.419660 & -0.42615\end{array}$ N $0.369234-2.012842 \quad-0.018829$ C $-0.635920-2.948086-0.458284$ C $-1.004387-2.798009-1.808587$ $\begin{array}{llll}\text { C }-1.218325 & -3.894794 & 0.402797\end{array}$ C $-2.002519-3.643386-2.299896$ $\begin{array}{llll}\text { C }-2.212751 & -4.714789 & -0.143169\end{array}$ C $-2.609900-4.596523-1.484185$ B $-0.274724-1.717644-2.760683$ F $1.111138-1.948108-2.886473$ F $-0.892860-1.56$ F $-0.892860-1.563094-3.994198$ $\begin{array}{llll}\text { F }-0.387569 & -0.355662 & -2.070249\end{array}$ $\begin{array}{llll}\text { C }-0.818382 & -4.067659 & 1.85525\end{array}$ $\begin{array}{llll}C & -0.276898 & -5.503444 & 2.13781\end{array}$ $\begin{array}{llll}C & -1.981212 & -3.754868 & 2.807085 \\ H & -0.006912 & -3.363765 & 2.083470\end{array}$ H $-0.006912-3.3637652 .083470$ H $0.560056-5.7603781 .469440$ H $0.074111-5.567484 \quad 3.180886$ $\begin{array}{llll}\text { H }-1.068742 & -6.257140 & 1.995715\end{array}$ H $-2.373921-2.7393892 .650350$ н $-1.645686-3.828105 \quad 3.852371$ H $-2.811748-4.463187 \quad 2.659001$ н $-2.296542-3.541554-3.345720$ H $-3.390048-5.251954-1.879545$ н $-2.687799 \quad-5.466684 \quad 0.488260$ C $3.155474 \quad 1.745854 \quad-1.227462$ C $3.335637 \quad 1.695357 \quad 1.235303$ $\begin{array}{llll}\text { C } 3.884563 & 2.935419 & -1.233533\end{array}$ $\begin{array}{llll}\text { C } 3.884563 & 2.935419 & -1.233533\end{array}$ $\begin{array}{llll}C & 4.053348 & 2.892675 & 1.172330\end{array}$ $\begin{array}{llll}\text { C } 4.325434 & 3.521081 & -0.044953\end{array}$ C $2.7615301 .074853-2.532340$ $\begin{array}{llll}C & 2.382513 & 2.063961 & -3.639397\end{array}$ $\begin{array}{llll}\text { C } 3.888628 & 0.149153 & -3.022292\end{array}$ $\begin{array}{llll}\text { H } & 1.888941 & 0.432376 & -2.346254 \\ \text { H } & 4.144772 & -0.610645 & -2.270527\end{array}$ $\begin{array}{llll}\text { H } & 4.144772 & -0.610645 & -2.270527\end{array}$ $\begin{array}{lllll}\text { H } & 4.798638 & 0.732194 & -3.239342\end{array}$ H $3.571621-0.375552-3.93577$ H $1.6518812 .809094-3.291339$ H $3.2625032 .604442-4.021707$ H $1.940177 \quad 1.514810-4.483428$ C $3.1040821 .054570 \quad 2.593571$ C $2.326261 \quad 1.978139 \quad 3.544032$ c $4.432804 \quad 0.645140 \quad 3.253532$ H 2.5049200 .1439202 .456869 $\begin{array}{llll}\text { H } & 5.039549 & -0.007339 \quad 2.609596\end{array}$ $\begin{array}{llll}\text { H } & 5.039549 & -0.007339 & 2.609596 \\ \text { H } & 4.231900 & 0.113989 & 4.196637\end{array}$ H 5.0388391 .5338393 .490185 H 1.4170252 .3763003 .075120 $\begin{array}{llll}\text { H } & 1.417025 & 2.376300 & 3.075120 \\ \text { H } 2.054318 & 1.426860 & 4.456969\end{array}$ $\begin{array}{llll}\text { H } & 2.054318 & 1.426860 & 4.456969 \\ \text { H } & 2.942750 & 2.842010 & 3.840044\end{array}$ $\begin{array}{llll}\text { H } & 2.942750 & 2.842010 & 3.840044 \\ \mathrm{H} & 4.111883 & 3.416895 & -2.184017\end{array}$ $\begin{array}{lllll}\text { H } & 4.111883 & 3.416895 & -2.184017\end{array}$ $\begin{array}{lllll}\text { H } & 4.881207 & 4.461510 & -0.067018 \\ \text { H } & 4.403704 & 3.348905 & 2.098666\end{array}$ $\begin{array}{lllll}\text { H } & 4.403704 & 3.348905 & 2.098666 \\ \text { C } & -0.241247 & 1.887317 & -0.564675\end{array}$ $\begin{array}{llll}\text { H } 0.559223 & 1.901930 & -1.319808\end{array}$ $\begin{array}{llll}\text { C } 0.062696 & 2.810422 & 0.485865\end{array}$ N $0.209004 \quad 3.566720 \quad 1.367494$ C $-1.5981662 .199565-1.210193$ c $-1.519312 \quad 3.457584 \quad-2.084956$ H $-2.3550592 .358289-0.426913$ H $-1.903968 \quad 1.342595-1.826030$ H $-0.818224 \quad 3.307677-2.919163$ $\mathrm{H}-0.818224 \quad 3.307677 \quad-2.919163$ $4-1.1861330 .328221-1.500335$

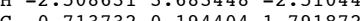
C -0.7137320 .1944041 .791872$ C $-1.734676-0.2044700 .894070$ C $-0.057269-0.686095 \quad 2.699709$ H $-0.655509 \quad 1.243224 \quad 2.102793$ $\begin{array}{lllll}\mathrm{H} & -2.458899 & 0.541987 & 0.571004\end{array}$ н $-2.064434-1.244685 \quad 0.836970$ N $0.521782-1.360797 \quad 3.458604$ 83

$16 \mathrm{R}=\mathrm{CHCNEt} \quad \mathrm{AC} / \mathrm{N}$

Ni $0.017852-0.014595 \quad 0.053970$ $\begin{array}{llll}\mathrm{N} & 1.926224 & -0.012807 & -0.059548\end{array}$ $\begin{array}{llll}\text { C } 2.767059 & 1.152499 & -0.027040\end{array}$ C $2.477338-1.189953-0.229532$ C $3.939371-1.444140-0.365184$ H $4.535832-0.554148-0.134766$ $\begin{array}{llll}\mathrm{H} & 4.535832 & -0.554148 & -0.134766 \\ \mathrm{H} & 4.159963 & -1.761540 & -1.398749\end{array}$ H $4.246200-2.2722770 .292706$ C $1.522893-2.296275-0.336919$

$\begin{array}{llll}\text { C } 1.970085 & -3.661607 & -0.736152\end{array}$ H $2.400841-3.617947-1.748518$ H $2.741159-4.035105-0.04423$ H $1.125660-4.359469-0.76103$ N $0.295418-1.940759-0.105794$ C $-0.794387-2.798977-0.451133$ C $-1.167998-2.781572-1.808921$ $\begin{array}{llll}\text { C }-1.480670 & -3.507969 & 0.548811\end{array}$ $\begin{array}{llll}\text { C }-2.265082 & -3.579665 & -2.155734\end{array}$ C $-2.562306-4.2954730 .133686$ C $-2.944785-4.337736-1.205029$ $\begin{array}{llll}C & -2.944785 & -4.337736 & -1.205029\end{array}$ B $-0.423469-1.812677-2.899881$ F $0.953138-2.169651-3.068952$ F $-1.090282-1.844930-4.132108$ $\begin{array}{llll}F & -0.433864 & -0.446720 & -2.387125\end{array}$ $\begin{array}{llll}C-1.040893 & -3.443223 & 1.999837\end{array}$ $\begin{array}{lll}\text { C }-0.032427 & -4.555058 & 2.332307\end{array}$ $\begin{array}{llll}\text { C }-2.211411 & -3.487749 & 2.987667\end{array}$ H $-0.528461-2.4756272 .136392$ H $0.860471-4.4979391 .694249$ H $0.291088-4.4805203 .382687$ н $-0.491681-5.545456 \quad 2.180706$ н $-2.971814 \quad-2.729777 \quad 2.749022$ $\mathrm{H}-1.846814 \quad-3.300498 \quad 4.009179$ н $-2.698864-4.474957 \quad 2.990594$ H $-2.581142-3.584724-3.201003$ H $-3.788548-4.965162-1.504292$ $\begin{array}{llll}\mathrm{H}-3.788548 & -4.965162 & -1.504292\end{array}$ $\begin{array}{lllll}\text { H } & -3.112835 & -4.881298 & 0.870487 \\ \text { C } 3.263128 & 1.675160 & -1.237380\end{array}$ $\begin{array}{llll}C & 3.263128 & 1.675160 & -1.237380\end{array}$ $\begin{array}{llll}\text { C } & 4.051411 & 2.830715 & -1.167834\end{array}$ $\begin{array}{llll}\text { C } 4.051411 & 2.830715 & -1.167834 \\ \text { C } 3.805627 & 2.911180 & 1.235080\end{array}$ $\begin{array}{llll}\text { C } & 3.805627 & 2.911180 & 1.235080 \\ \text { C } & 4.329828 & 3.447041 & 0.047130\end{array}$ $\begin{array}{llll}\text { C } & 4.329828 & 3.447041 & 0.047130 \\ \text { C } & 2.949689 & 1.065062 & -2.594574\end{array}$ $\begin{array}{llll}\text { C } & 2.949689 & 1.065062 & -2.594574 \\ \text { C } & 2.049195 & 1.996522 & -3.420777\end{array}$ C $4.2270120 .728603-3.379820$ $\begin{array}{lllll}\text { H } & 2.383274 & 0.134262 & -2.452097\end{array}$ H $4.921742 \quad 0.109607 \quad-2.792673$ H $4.765446 \quad 1.642058-3.678026$ $\begin{array}{lllll}\text { H } & 3.963753 & 0.180126 & -4.296727\end{array}$ H $1.108761 \quad 2.206513-2.894700$ H $2.5540602 .953301-3.630122$ H $1.796412 \quad 1.516375-4.377840$ $\begin{array}{llll}\text { H } & 1.796412 & 1.516375 & -4.377840\end{array}$ $\begin{array}{llll}\text { C } 2.465489 & 1.172726 & 2.508240\end{array}$ $\begin{array}{llll}\text { C } & 2.224394 & 2.216192 & 3.601338\end{array}$ C 3.3811460 .0563593 .038201 $\begin{array}{llll}\text { H } & 1.489829 & 0.716955 & 2.264606 \\ \text { H } 3.497039 & -0.762556 & 2.314213\end{array}$ $\begin{array}{llll}\text { H } & 3.497039 & -0.762556 & 2.314213 \\ \text { H } & 2.963508 & -0.366329 & 3.965256\end{array}$ $\begin{array}{llll}\text { H } & 2.963508 & -0.366329 & 3.965256 \\ \text { H } & 4.383140 & 0.457699 & 3.261634\end{array}$ $\begin{array}{llll}\mathrm{H} & 4.383140 & 0.457699 & 3.261634 \\ \mathrm{H} & 1.608366 & 3.049492 & 3.236392\end{array}$ $\begin{array}{lllll}\mathrm{H} & 1.608366 & 3.049492 & 3.236392 \\ \mathrm{H} & 1.688120 & 1.748430 & 4.440165\end{array}$ $\begin{array}{llll}\mathrm{H} & 1.688120 & 1.748430 & 4.440165 \\ \mathrm{H} & 3.172792 & 2.611778 & 3.997723\end{array}$ H $4.447938 \quad 3.257020 \quad-2.089610$ H $4.944448 \quad 4.350282 \quad 0.080158$ H $4.012339 \quad 3.410551 \quad 2.180228$ C $-0.218701 \quad 1.958869-0.021265$ $\begin{array}{llll}\text { H } & 0.783633 & 2.354803 & -0.253045\end{array}$ $\begin{array}{llll}\text { C }-0.610146 & 2.419846 & 1.281847\end{array}$ $\begin{array}{llll}\text { N }-0.964222 & 2.779871 & 2.338721\end{array}$ C $-1.2171782 .345316-1.123106$ C $-1.1842973 .847962-1.420074$ $\mathrm{H}-2.2333112 .049603-0.821547$ $\begin{array}{llll}\mathrm{H} & -2.233311 & 2.049603 & -0.821547 \\ \mathrm{H} & -0.979710 & 1.768658 & -2.029083\end{array}$ H $-0.9797101 .768658-2.029083$ $\begin{array}{llll}\mathrm{H} & -0.184889 & 4.164054 & -1.757048 \\ \mathrm{H} & -1.441118 & 4.433851 & -0.524087\end{array}$ $\begin{array}{llll}\mathrm{H} & -1.441118 & 4.433851 & -0.524087 \\ \mathrm{H} & -1.904790 & 4.095395 & -2.214376\end{array}$ $\begin{array}{lllr}\mathrm{H} & -1.904790 & 4.095395 & -2.214376 \\ \mathrm{~N} & -1.709187 & -0.146548 & 0.569050\end{array}$ $\begin{array}{llll}\mathrm{N} & -1.709187 & -0.146548 & 0.569050 \\ \mathrm{C} & -2.830481 & -0.252621 & 0.859649\end{array}$ $\begin{array}{lll}C-4.191068 & -0.357527 & 1.213871\end{array}$ $\begin{array}{llll}\text { C }-5.001784 & -1.278492 & 0.655746\end{array}$ H $-4.548050 \quad 0.357069 \quad 1.959359$ H $-6.051067-1.3305590 .947079$ H $-4.627945-1.981054-0.091041$ 81

$16 \mathrm{R}=\mathrm{n}-\mathrm{Pr} \mathrm{ET} / \mathrm{pi}$

$\mathrm{Ni}-0.001676 \quad 0.000403 \quad 0.001767$ $\begin{array}{llllll}\mathrm{N} & 1.992424 & 0.001208 & 0.000647\end{array}$ $\begin{array}{llll}C & 2.818537 & 1.173066 & -0.001180\end{array}$ C $2.549022-1.177365-0.078714$ C $4.016123-1.440448-0.146326$ $\begin{array}{lllll}\text { H } 4.601667 & -0.535387 & 0.056195\end{array}$ H $4.277609-1.806972-1.153704$ H $4.305609-2.2309280 .563863$ C $1.600205-2.298693-0.202353$ C $2.088015-3.656076-0.577610$ H $2.623537-3.600575-1.538459$ H $2.786189-4.044390 \quad 0.181082$ H $1.250258-4.353576-0.695987$ $\mathrm{N} 0.355901-1.968041-0.046327$ C $-0.689008-2.858890-0.461973$ 
F $1.032662-1.904161-2.943818$ F $-0.993302-1.463951-3.993340$ F $-0.387320-0.268425-2.089935$ C $-0.876316-3.8911601 .875400$ C $-0.312592-5.292054 \quad 2.15861$ $\begin{array}{llll}\text { C }-2.018370 & -3.567953 & 2.850073\end{array}$ H $-0.065898 \quad-3.1657892 .053037$ H $0.525823-5.5336501 .488694$ $\mathrm{H}-1.088085-6.0605052 .013759$ H $0.041836-5.3615013 .199181$ $\begin{array}{llll}\text { H } & 0.041836 & -5.361501 & 3.199181\end{array}$ $\begin{array}{llll}\mathrm{H}-2.839918 & -4.293886 & 2.745474\end{array}$ $\begin{array}{llll}\mathrm{H} & -2.432607 & -2.565415 & 2.668873\end{array}$ $\begin{array}{llll}\mathrm{H}-1.659880 & -3.615813 & 3.890527\end{array}$ $\mathrm{H}-2.472944-3.368553-3.284304$ H $-3.608197-4.998227-1.768300$ $\begin{array}{llll}\text { H }-2.873703 & -5.223293 & 0.577268\end{array}$ C $3.2780321 .680615-1.232915$ C $3.0752801 .830625 \quad 1.218276$ $\begin{array}{lllll}\text { C } 4.023714 & 2.864704 & -1.207887\end{array}$ C $3.8346963 .002122 \quad 1.186891$ $\begin{array}{llll}\text { C } 4.308160 & 3.520580 & -0.014341\end{array}$ C $2.987016 \quad 1.007432-2.564763$ C $2.1037581 .886017-3.463956$ $\begin{array}{llll}\text { C } 4.284509 & 0.634188 & -3.299925\end{array}$ H $2.4232620 .082249-2.385086$ H $1.1427552 .111498-2.982346$ $\begin{array}{llll}\text { H } & 1.142755 & 2.111498 & -2.982346\end{array}$ H $2.6059632 .835568-3.710109$ H $1.8893721 .352977-4.402110$ H $4.9603200 .040685-2.665609$ $\begin{array}{lllll}\text { H } & 4.833855 & 1.533829 & -3.621295 \\ \text { H } & 4.044833 & 0.045650 & -4.198590\end{array}$ $\begin{array}{llll}\text { H } & 4.044833 & 0.045650 & -4.198590 \\ \text { C } & 2.574479 & 1.263512 & 2.536176\end{array}$ $\begin{array}{llll}\text { C } & 2.574479 & 1.263512 & 2.536176\end{array}$ $\begin{array}{llll}\text { C } 3.565215 & 0.242786 & 3.119747\end{array}$ C $2.268842 \quad 2.338435 \quad 3.584554$ H $4.5385780 .722300 \quad 3.314029$ H $3.734961-0.601577 \quad 2.437884$ H $3.183009-0.159662 \quad 4.071263$ н $3.190703 \quad 2.802107 \quad 3.970169$ H $1.628191 \quad 3.135285 \quad 3.179257$ H $1.752216 \quad 1.882952 \quad 4.443281$ H $4.386265 \quad 3.280608 \quad-2.150073$ H $4.894230 \quad 4.442897 \quad-0.021351$ H $4.051477 \quad 3.5270722 .119015$ $\begin{array}{llll}\text { H } & 4.051477 & 3.527072 & 2.119015 \\ \text { C } & -0.238091 & 1.927064 & -0.312891\end{array}$ $\begin{array}{llll}\text { C } & -0.0 .983674 & 1.871224 & -1.125245\end{array}$ $\begin{array}{lllll}\mathrm{H} & -0.983674 & 1.871224 & -1.125245 \\ \mathrm{H} & 0.742580 & 2.199044 & -0.732086\end{array}$ $\begin{array}{llll}\mathrm{H} & -0.640844 & 2.920282 & 0.754779\end{array}$ $\begin{array}{llll}\text { C } & -0.640844 & 2.920282 & 0.754779 \\ \text { C }-0.944919 & 0.004016 & 1.766208\end{array}$ $\begin{array}{llll}\text { C } & -0.944919 & 0.004016 & 1.766208 \\ \text { C } & -1.877721 & -0.101966 & 0.724047\end{array}$ $\begin{array}{llll}\mathrm{C} & -1.877721 & -0.101966 & 0.724047 \\ \mathrm{H}-0.803634 & 0.947657 & 2.295711\end{array}$ $\begin{array}{llll}\mathrm{H} & -0.803634 & 0.947657 & 2.295711 \\ \mathrm{H}-0.620586 & -0.888777 & 2.307923\end{array}$ $\begin{array}{llll}\mathrm{H} & -0.620586 & -0.888777 & 2.307923 \\ \mathrm{H} & -2.468192 & 0.764633 & 0.418905\end{array}$ H $-2.468192 \quad 0.7646330 .418905$ H $-2.284077 \quad-1.073041 \quad 0.425006$ H $1.636200 \quad 0.730007 \quad 2.315958$ $\begin{array}{llll}\text { C } & -0.736955 & 4.340286 & 0.173217\end{array}$ H $0.102425 \quad 2.928103 \quad 1.568933$ H $-1.614317 \quad 2.661422 \quad 1.204249$ H $-1.031627 \quad 5.0623250 .951133$ H $0.232100 \quad 4.656925-0.242216$ H $-1.483191 \quad 4.382445 \quad-0.635451$ 82

$16 \mathrm{R}=\mathrm{n}-\mathrm{Pr} \mathrm{AC} / \mathrm{p}$

Ni $-0.006018 \quad 0.004394 \quad 0.004058$

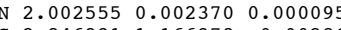
$\begin{array}{llll}\text { C } 2.846331 & 1.166378 & -0.002869\end{array}$ $\begin{array}{llll}\text { C } 2.534869 & -1.184540 & 0.090360\end{array}$ $\begin{array}{llll}\text { C } 3.987414 & -1.467742 & 0.277740\end{array}$ $\begin{array}{llll}\text { H } 4.589626 & -0.552776 & 0.227708\end{array}$ H $4.347287-2.177923-0.481786$ H $4.143844-1.9454361 .260592$ $\begin{array}{llll}\text { C } 1.585164 & -2.308224 & -0.057630\end{array}$ $\begin{array}{llll}\text { C } 2.095077 & -3.674537 & -0.363172\end{array}$ H $2.717812-3.637012-1.271678$ H $2.718331-4.055344 \quad 0.461342$ H $1.266262-4.367838-0.546653$ N $0.335444-1.973796-0.011397$ C $-0.699611-2.887039-0.413787$ $\begin{array}{llll}\text { C } & -0.699611 & -2.887039 & -0.413787 \\ \text { C } & -1.172741 & -2.672074 & -1.723300\end{array}$ $\begin{array}{llll}C & -1.172741 & -2.672074 & -1.723300\end{array}$ $\begin{array}{llll}C & -2.220150 & -3.484776 & -2.161825\end{array}$ $\begin{array}{llll}C & -2.220150 & -3.484776 & -2.161825 \\ C & -2.287096 & -4.633154 & -0.043409\end{array}$ $\begin{array}{llll}\text { C } & -2.287096 & -4.633154 & -0.043409 \\ \text { C }-2.777488 & -4.458036 & -1.332214\end{array}$ $\begin{array}{llll}\text { C } & -2.777488 & -4.458036 & -1.332214 \\ \text { B } & -0.512361 & -1.551350 & -2.685787\end{array}$ $\begin{array}{llll}\text { B }-0.512361 & -1.551350 & -2.685787 \\ \text { F } 0.866832 & -1.771093-2.903633\end{array}$ F $0.866832-1.771093-2.903633$ F $-1.205398-1.382157-3.879075$ F $-0.592412-0.211051-1.964498$ C $-0.740034-4.068532 \quad 1.873968$ $\begin{array}{llll}\text { C }-0.199272 & -5.492338 & 2.078971\end{array}$ $\begin{array}{llll}\text { C }-1.838517 & -3.771166 & 2.906075\end{array}$ H $0.082718-3.3645292 .066196$ н $0.593910-5.738043 \quad 1.357507$ H $0.211429-5.597640 \quad 3.095236$ H $-1.000064 \quad-6.239040 \quad 1.960719$ $\mathrm{H}-1.000064-6.2390401 .960719$ H $-2.237429-2.7527272 .793001$ H $-1.434518-3.8622113 .925627$ $\begin{array}{llll}\mathrm{H} & -2.677311 & -4.477444 & 2.800785 \\ \mathrm{H} & -2.597455 & -3.338267 & -3.175240\end{array}$
H $-3.598493-5.081517-1.689161$ H $-2.730343-5.3$ C $3.151608 \quad 1.740179-1.256009$ C $3.289192 \quad 1.737309 \quad 1.205151$ $\begin{array}{llll}\text { C } 3.928517 & 2.900532 & -1.273635\end{array}$ C $4.055722 \quad 2.9038331 .135827$ C $4.3822243 .490335-0.090523$ C $2.7065381 .092959-2.555498$ $\begin{array}{llll}\text { C } 2.242193 & 2.109084 & -3.605060\end{array}$ C $3.8242540 .209526-3.1$ $\begin{array}{llll}\text { C } 3.824254 & 0.209526 & -3.134498\end{array}$ $\begin{array}{lllll}\mathrm{H} & 1.856936 & 0.429774 & -2.341321\end{array}$ $\begin{array}{llll}\text { H } & 1.503679 & 2.810965 & -3.190516\end{array}$ H $1.7751631 .576506-4.446394$ $\begin{array}{llll}\mathrm{H} & 3.083317 & 2.695179 & -4.007464 \\ \mathrm{H} & 4.147746 & -0.557488 & -2.416133\end{array}$ $\begin{array}{llll}\text { H } 4.147746 & -0.557488 & -2.416133\end{array}$ $\begin{array}{lllll}\text { H } 3.463538 & -0.304147 & -4.03809\end{array}$ $\begin{array}{lllll}\text { H } & 4.704440 & 0.819161 & -3.397295\end{array}$ $\begin{array}{llll}\text { C } 2.960499 & 1.148768 & 2.565025\end{array}$ $\begin{array}{llll}\text { C } 2.102810 & 2.113901 \quad 3.39811\end{array}$ $\begin{array}{llll}\text { C } 4.225989 & 0.761702 & 3.345796\end{array}$ H $2.373664 \quad 0.232678 \quad 2.417032$ H $1.211066 \quad 2.439938 \quad 2.843248$ H $2.673222 \quad 3.017497 \quad 3.666350$ H $1.776871 \quad 1.623911 \quad 4.328012$ H $4.869109 \quad 0.080520 \quad 2.769501$ H 4.8237061 .651 $\begin{array}{lllll}\text { H } & 4.823706 & 1.651441 & 3.598884\end{array}$ $\begin{array}{lllll}\text { H } & 3.946739 & 0.262657 & 4.286274\end{array}$ $\begin{array}{lllll}\text { H } & 4.180955 & 3.358065 & -2.229891 \\ \text { H } & 4.984167 & 4.401949 & -0.123717\end{array}$ $\begin{array}{lllll}\text { H } & 4.984167 & 4.401949 & -0.123717\end{array}$ $\begin{array}{lllll}H & 4.407606 & 3.364814 & 2.059641\end{array}$ C $-0.1079881 .947941-0.30028$ $\begin{array}{llll}\mathrm{H} & 0.557288 & 2.418284 & 0.441960\end{array}$ H $0.382053 \quad 1.936030 \quad-1.286588$ $\begin{array}{llll}\text { C } & -1.458607 & 2.621878 & -0.380502\end{array}$ $\begin{array}{llll}\text { C }-1.764540 & -0.167463 & 0.924379\end{array}$ C $-0.772621 \quad 0.277504 \quad 1.833039$ H $-2.069349-1.216334 \quad 0.896821$ H $-2.494080 \quad 0.5481590 .548746$ H $-0.754565 \quad 1.330657 \quad 2.127895$ C $-0.123729 \quad-0.578290 \quad 2.771091$ N $0.444843-1.238923 \quad 3.550546$ C $-1.312004 \quad 4.072668-0.870335$ H -1.9599562 .6401310 .602178$ $\begin{array}{llllll}\mathrm{H}-1.95956 & 2.640131 & 0.602178\end{array}$ $\begin{array}{lllll}\mathrm{H} & -2.110874 & 2.076084 & -1.081318\end{array}$ $\mathrm{H}-2.295360-4.565340-0.91401$ $\begin{array}{lllll}\mathrm{H} & -0.662715 & 4.654331 & -0.198743\end{array}$ 82

$16 \mathrm{R}=\mathrm{n}-\mathrm{Pr} \mathrm{AC} / \mathrm{N}-\mathrm{t}$

Ni $-0.023240-0.057022-0.002550$ $\begin{array}{llll}\mathrm{N} & 1.888023 & -0.011950 & 0.014013\end{array}$ C $2.707848 \quad 1.172367 \quad 0.026876$ C $2.477570-1.170683-0.160409$ C $3.945585-1.376831-0.298401$ н $4.517362-0.472535-0.059540$ H $4.168232-1.671009-1.338966$ $\begin{array}{lllll}\text { H } & 4.281045 & -2.206549 & 0.343037\end{array}$ $\begin{array}{llll}C & 1.563769 & -2.310367 & -0.324818\end{array}$ C 2.086155 $\begin{array}{llll}\text { C } 2.086155 & -3.671447 & -0.628308\end{array}$ H $2.608764-3.656163-1.597416$ $\begin{array}{lll}\text { H } 2.796935 & -3.995829 & 0.148421\end{array}$ H $1.267142-4.396928-0.702196$ $\begin{array}{llll}\mathrm{N} & 0.311473 & -1.978021 & -0.241118 \\ \mathrm{C} & -0.697708 & -2.892595 & -0.689689\end{array}$ $\begin{array}{llll}C & -0.899434 & -2.928639 & -2.082305\end{array}$ C $-1.460100-3.619414 \quad 0.235220$ $\begin{array}{llll}\text { C }-1.882013 & -3.814992 & -2.538446\end{array}$ $\begin{array}{llll}\text { C }-2.422097 & -4.494898 & -0.282802\end{array}$ $\begin{array}{llll}\text { C }-2.622525 & -4.605087 & -1.657527\end{array}$ в $-0.093573-1.929109-3.107255$ F $-0.584423-2.072910-4.416426$ F $-0.274803-0.566540-2.666063$ F $1.320473-2.193083-3.084414$ C $-1.226577-3.490374 \quad 1.729988$ C $-0.390901-4.660575 \quad 2.271003$ C $-2.533183-3.3552042 .520208$ C $-2.533183-3.3552042 .520208$ H $-0.648991-2.564271 \quad 1.886736$ H $0.579044-4.7344461 .757705$ H $-0.202854-4.5367513 .349536$ H $-0.922781-5.6142002 .121669$ H $-3.151150-2.532398 \quad 2.13102$ $\begin{array}{llll}\mathrm{H} & -2.315595 & -3.154377 & 3.58081\end{array}$ $\begin{array}{llll}\mathrm{H} & -3.128614 & -4.280129 & 2.475142 \\ \mathrm{H} & -2.057464 & -3.870381 & -3.614804\end{array}$ $\begin{array}{llll}\mathrm{H} & -2.057464 & -3.870381 & -3.614804 \\ \mathrm{H} & -3.367727 & -5.305393 & -2.039996\end{array}$ H $-3.367727-5.305393 \quad-2.039996$ $\begin{array}{lllll}\mathrm{H} & -3.021118 & -5.100643 & 0.398894\end{array}$ C $3.120894 \quad 1.721599-1.201609$ C $3.025096 \quad 1.766582 \quad 1.264083$ $\begin{array}{llll}\text { C } 3.895309 & 2.887586 & -1.162136\end{array}$ C $3.803652 \quad 2.9231661 .252736$ C $4.246545 \quad 3.487796 \quad 0.042527$ $\begin{array}{llll}\text { C } 2.728303 & 1.127167 & -2.543136\end{array}$ $\begin{array}{llll}\text { C } 1.742160 & 2.048367 & -3.278175\end{array}$ $\begin{array}{llll}\text { C } 3.952616 & 0.835385 & -3.423652\end{array}$ $\begin{array}{llll}\text { H } 2.200717 & 0.177576 & -2.382946\end{array}$ H $0.8485892 .236931-2.667223$ H $2.2116773 .015280-3.522640$

H $1.413853 \quad 1.566766 \quad-4.210649$ H $4.701422 \quad 0.225578-2.895505$ H $4.446543 \quad 1.764638-3.749476$ H $3.6347440 .287475-4.323090$ $\begin{array}{llll}\text { C } 2.553796 & 1.155239 & 2.572151\end{array}$ $\begin{array}{llll}\text { C } 2.246819 & 2.203431 & 3.646891\end{array}$ C $3.571177 \quad 0.136006 \quad 3.110048$ H $1.619468 \quad 0.6111292 .353189$ н $3.742995-0.6850532 .400038$ H $4.539318 \quad 0.627158 \quad 3.301039$ H $3.212208-0.299839 .301039$ H $3.212208-0.2998390507057$ H $1.587967 \quad 2.996168 \quad 3.264247$ 3.1670572 .6745194 .026590 4.7509601 .7236824 .504458 $\begin{array}{llll}\text { H } 4.225450 & 3.334026 & -2.100563\end{array}$ $\begin{array}{lllll}\text { H } & 4.853418 & 4.396897 & 0.052203\end{array}$ H $4.068536 \quad 3.4072392 .192276$ C $-0.291820 \quad 1.872508 \quad 0.202142$ H $0.693136 \quad 2.349913 \quad 0.087972$ $\begin{array}{llll}\text { H } & -0.918687 & 2.131383 & -0.669713\end{array}$ C $-0.953341 \quad 2.292475 \quad 1.507147$ $\begin{array}{llll}\mathrm{N} & -1.808542 & -0.226195 & 0.060149\end{array}$ $\begin{array}{llll}\text { C }-2.952994 & -0.398810 & -0.069814\end{array}$ C $-4.340785-0.600152-0.21720$ $\begin{array}{lllll}\text { H } & -4.990208 & 0.112515 & 0.297552\end{array}$

C $-4.839202-1.599885-0.964862$ H $-4.182458-2.301044-1.482713$ H $-5.918069-1.721072-1.065372$ C $-1.0749393 .818989 \quad 1.619569$ $\begin{array}{llll}\mathrm{H} & -0.374455 & 1.917726 & 2.367869\end{array}$ H $-1.956529 \quad 1.845399 \quad 1.594834$ н $-1.545888 \quad 4.109795 \quad 2.571864$ $\begin{array}{llll}\mathrm{H} & -0.085411 & 4.298504 & 1.564744\end{array}$ $\mathrm{H}-1.685121 \quad 4.223972 \quad 0.797198$ 82

$16 \quad \mathrm{R}=\mathrm{n}-\mathrm{Pr} \quad \mathrm{AC} / \mathrm{N}-\mathrm{C}$

Ni $0.003309-0.050801-0.085010$ N $1.975252-0.019475-0.055530$

$\begin{array}{lllll}\text { C } 2.757771 & 1.177584 & -0.032237\end{array}$ $\begin{array}{llll}\text { C } 2.534701 & -1.194925 & -0.008306\end{array}$ $\begin{array}{llll}\text { C } 3.999269 & -1.457741 & 0.059285\end{array}$ H $4.572645-0.5252120 .128627$ H $4.316506-2.002732-0.845351$ H $4.242545-2.0992060 .921605$ $\begin{array}{llll}\text { C } 1.574719 & -2.301606 & -0.115436\end{array}$ $\begin{array}{llll}\text { C } 2.574719 & -2.301606 & -0.115436 \\ \text { C } 2.059268 & -3.696085 & -0.308877\end{array}$ H $2.572540-3.763700-1.281663$ H $2.774477-3.968502 \quad 0.483405$ $\begin{array}{llll}\text { H } & 1.228975 & -4.411428 & -0.321074\end{array}$ N $0.319428-1.934125 \quad-0.087837$ C $-0.668846-2.884398-0.536674$ C $-0.810941-2.972968-1.934058$ $\begin{array}{llll}\text { C }-1.440818 & -3.610184 & 0.382847\end{array}$ $\begin{array}{llll}\text { C }-1.774898 & -3.874113 & -2.399189\end{array}$ $\begin{array}{llll}\text { C }-2.381646 & -4.501248 & -0.146672\end{array}$ $\begin{array}{llll}\text { C }-2.545741 & -4.637146 & -1.522554\end{array}$ B $0.057029-2.040441-2.967263$ F $1.460426-2.330560-2.879565$ F $-0.388267-2.215424-4.286267$ $\begin{array}{llll}F & -0.388267 & -2.215424 & -4.286267\end{array}$ $\begin{array}{llll}\text { F }-0.099955 & -0.644436 & -2.592912\end{array}$ $\begin{array}{llll}C & -1.250095 & -3.470691 & 1.88415\end{array}$ $\begin{array}{llll}C & -0.419304 & -4.630438 & 2.456836\end{array}$ $\begin{array}{cc}C-2.580260-3.354516 & 2.639588\end{array}$ H $-0.685815-2.5389502 .056212$ H $0.571712 \quad-4.692901 \quad 1.984876$ H $-0.277162-4.503356 \quad 3.542064$ H $-0.933155-5.590550 \quad 2.287876$ H $-3.206861-2.547655 \quad 2.232142$ H $-2.392564-3.1443913 .704099$ H $-3.154298-4.292241 \quad 2.585830$ H $-1.913918-3.962207-3.478231$ H $-3.286984-5.337832-1.910608$ н $-2.998583 \quad-5.0952310 .528866$ C $3.1472681 .744027-1.260768$ $\begin{array}{lllll}C & 3.040840 & 1.793932 & 1.202054\end{array}$ C $3.8765072 .937920-1.226140$ $\begin{array}{llll}\text { C } 3.876507 & 2.937920 & -1.226140\end{array}$ $\begin{array}{llll}\text { C } 3.771118 & 2.983785 & 1.187823\end{array}$ $\begin{array}{llll}\text { C } & 4.198826 & 3.558328 & -0.021834 \\ \text { C } & 2.800428 & 1.096971 & -2.589507\end{array}$ $\begin{array}{llll}\text { C } 1.901447 & 2.010032 & -3.434695\end{array}$ $\begin{array}{llll}\text { C } 4.061692 & 0.696720 & -3.368959\end{array}$ $\begin{array}{lllll}\text { C } & 4.061692 & 0.696720 & -3.368959 \\ \text { H } & 2.223755 & 0.182115 & -2.398904\end{array}$ $\begin{array}{lllll}\text { H } & 2.223755 & 0.182115 & -2.398904 \\ \text { H } & 0.984579 & 2.273510 & -2.887628\end{array}$ $\begin{array}{lllll}\text { H } & 0.984579 & 2.273510 & -2.887628 \\ \text { H } & 2.423583 & 2.938576 & -3.717993\end{array}$ H $1.6013571 .483996-4.352773$ H $4.720707 \quad 0.051903-2.767820$ H $4.642726 \quad 1.581555 \quad-3.675554$ H $3.7751110 .142814 \quad-4.275294$ C $2.590030 \quad 1.195088 \quad 2.523550$ $\begin{array}{llll}\text { C } 1.736857 & 2.181730 & 3.332079\end{array}$ 
H $4.201068 \quad 3.386158 \quad-2.166157$ H $4.781462 \quad 4.483290-0.013781$ H $4.019012 \quad 3.474447 \quad 2.130010$ C $-1.922048 \quad-0.204075 \quad 0.275070$ C $-2.755557 \quad 0.039853-0.969889$ H $-2.066563 \quad-1.224240 \quad 0.653844$ H $-2.142880 \quad 0.521559 \quad 1.075907$ $\mathrm{N}-0.221952 \quad 1.721669-0.062046$ C $-0.32257422 .882750-0.117029$ c $-0.476032+4281897-0.169955$ $\begin{array}{llll}C & -0.476032 & 4.281897 & -0.169955\end{array}$ C $0.5657505 .130440-0.128180$ H $-1.504448 \quad 4.644472 \quad-0.25066$ $\begin{array}{llll}\mathrm{H} & 1.591090 & 4.765302 & -0.053161\end{array}$ H $0.3967606 .206219-0.171067$ $\begin{array}{llll}1.953566 & 0.326440 & 2.297804\end{array}$ $\begin{array}{llll}C & -4.244581 & -0.230080 & -0.702669\end{array}$ $\mathrm{H}-2.414320-0.614145-1.786780$ H $-2.6260891 .076347-1.323661$ H $-4.840569-0.037756-1.608466$ H $-4.400151-1.281100-0.414003$ H $-4.630979 \quad 0.409438 \quad 0.107105$

69

$17 \mathrm{R}=\mathrm{Me}$

$\mathrm{Ni}-0.002568 \quad 0.013596 \quad 0.201909$ $\begin{array}{lllll}\mathrm{N} & 1.841108 & 0.009827 & 0.010293\end{array}$ $\begin{array}{llll}\text { C } 2.694182 & 1.170891 & 0.027647\end{array}$ C $2.371304-1.151138-0.343302$ $\begin{array}{llll}\text { C } 2.371304-1.151138 & -0.343302\end{array}$ C $3.798884-1.363707-0.725868$ $\begin{array}{llll}\text { H } 4.386346 & -0.441894 & -0.640956\end{array}$ H $3.869849-1.729470-1.763628$ $\begin{array}{lllll}\text { H } & 4.258008 & -2.135774 & -0.086352\end{array}$ C $1.445279-2.275002-0.30464$ C $1.864670-3.629271-0.769858$ H $2.306195-3.547621-1.776203$ H $2.630330-4.074938-0.115148$ H $1.004836-4.308153-0.82455$ $\begin{array}{llll}\text { N } 0.247778 & -1.944536 & 0.111333\end{array}$ $\begin{array}{lllll}\text { C } & -0.782495 & -2.882967 & 0.341536\end{array}$ $\begin{array}{llll}\text { C }-2.023341 & -2.624206 & -0.273612\end{array}$ $\begin{array}{llll}\text { C }-0.653574 & -3.971432 & 1.232028\end{array}$ $\begin{array}{llll}\text { C }-3.086493 & -3.503526 & -0.128552\end{array}$ $\begin{array}{llll}\text { C }-1.742752 & -4.842348 & 1.354363\end{array}$ $\begin{array}{llll}\text { C }-2.931636 & -4.639261 & 0.664176\end{array}$ S $-2.275679-1.068224-1.170341$ $0-1.346817-1.058247-2.303223$ $0-3.708959-0.978134-1.420936$ $0-1.874252-0.040516-0.072245$ $\begin{array}{llll}\text { C } 0.534694 & -4.138802 & 2.164941\end{array}$ $\begin{array}{llll}\text { C } & 1.074572 & -5.573617 & 2.209720\end{array}$ $\begin{array}{llll}\text { C } 0.144821 & -3.659372 & 3.574743\end{array}$ H $1.345954-3.4820291 .822791$ H $1.312887-5.956057 \quad 1.206254$ H $1.987118 \quad-5.613443 \quad 2.824231$ H $0.345652-6.260224 \quad 2.666420$ H $-0.216015 \quad-2.620420 \quad 3.551019$ H $-0.660170-4.286892 \quad 3.988558$ H $1.010964-3.718294 \quad 4.252500$ H $-4.028908-3.271453-0.623829$ $\begin{array}{llll}\text { H } & -3.754639 & -5.345312 & 0.775129\end{array}$ $\begin{array}{llll}\mathrm{H} & -3.754639 & -5.345312 & 0.775129 \\ \mathrm{H} & -1.660475 & -5.696495 & 2.026573\end{array}$ C $2.851953 \quad 1.934571-1.141553$

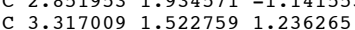
C $3.7016663 .044116-1.078656$ $\begin{array}{llll}\text { C } & 3.701666 & 3.044116 & -1.078656\end{array}$ $\begin{array}{llll}\text { C } & 4.154523 & 2.639952 & 1.248339 \\ \text { C } & 4.358367 & 3.393637 & 0.098659\end{array}$ $\begin{array}{llll}\text { C } 4.358367 & 3.393637 & 0.098659 \\ \text { C } 2.126120 & 1.604010 & -2.433756\end{array}$ $\begin{array}{llll}\text { C } 2.126120 & 1.604010 & -2.433756 \\ \text { C } 3.098097 & 1.123343 & -3.522302\end{array}$ $\begin{array}{llll}\text { C } 3.098097 & 1.123343 & -3.522302 \\ \text { C } 1.308219 & 2.800272 & -2.941957\end{array}$ $\begin{array}{llll}\text { C } 1.308219 & 2.800272 & -2.941957\end{array}$ H $1.414737 \quad 0.789771-2.227985$ H $3.8061301 .922555-3.794834$ H $2.540203 \quad 0.838926-4.427632$ H $3.686395 \quad 0.254663 \quad-3.192435$ H $0.6356953 .181282-2.160853$ H $0.6987802 .494379-3.805806$ $\begin{array}{lllll}\text { H } & 1.963778 & 3.624860 & -3.264573\end{array}$ $\begin{array}{llll}\text { H } & 1.963778 & 3.624860 & -3.264573 \\ \text { C } 3.090747 & 0.737032 & 2.516690\end{array}$ C $2.4506881 .616893 \quad 3.600390$ $\begin{array}{llll}\text { C } 4.390440 & 0.096326 & 3.024791\end{array}$ $\begin{array}{llll}\text { H } 2.379381 & -0.071759 & 2.292659\end{array}$ $\begin{array}{lllll}\mathrm{H} & 1.508346 & 2.058189 & 3.244550\end{array}$ H $3.1230742 .437916 \quad 3.894766$ H 2.2370281 .0172204 .498719 $\begin{array}{lllll}\text { H } & 4.848566 & -0.548788 & 2.259662\end{array}$ H $5.129613 \quad 0.865774 \quad 3.298625$ H $4.191794-0.512397 \quad 3.920587$ H $3.8472293 .653330-1.974234$ H $5.019192 \quad 4.263570 \quad 0.119206$ H $4.652096 \quad 2.926496 \quad 2.177486$ C $-0.233392 \quad 1.902270 \quad 0.457092$ H $-0.930191 \quad 1.951750 \quad 1.311002$ H $-0.7326932 .295903 \quad-0.440366$ $\begin{array}{llll}\text { H } & 0.706877 & 2.429787 & 0.663834\end{array}$ 75

$17 \mathrm{R}=\mathrm{Me} \mathrm{ET} / \mathrm{pi}$

$\begin{array}{lll} & 0.027695\end{array}$ $\mathrm{N} 1.986483 \quad 0.001681 \quad 0.036176$ $\begin{array}{llll}\text { C } 2.830480 & 1.163218 & 0.011850\end{array}$ C 2.547666 $\begin{array}{llll}\text { C } 4.016617 & -1.444338 & -0.048214\end{array}$ H $4.599666-0.530104 \quad 0.115399$ H $4.284305-1.853532-1.037183$ H $4.305031-2.199943 \quad 0.699413$ $\begin{array}{llll}\text { C } 1.607267 & -2.302611 & -0.076688\end{array}$ C $2.101407-3.676269-0.381177$ H $2.700400-3.655089-1.304516$ $\begin{array}{llll}\text { H } 2.743899 & -4.049251 & 0.432972\end{array}$ $\begin{array}{lllll}\mathrm{H} & 1.268302 & -4.373087 & -0.53239\end{array}$ $\begin{array}{lllll}\mathrm{N} & 0.355571 & -1.968571 & 0.061213\end{array}$ $\begin{array}{llll}C & -0.668331 & -2.890045 & -0.307110\end{array}$ $\begin{array}{llll}C & -1.120362 & -2.779176 & -1.633451\end{array}$ $\begin{array}{llll}C & -1.242977 & -3.809336 & 0.580374\end{array}$ $\begin{array}{llll}\text { C }-2.138194 & -3.602530 & -2.096345\end{array}$ $\begin{array}{llll}C & -2.267488 & -4.627439 & 0.085062\end{array}$ C $-2.708835-4.535226-1.230050$ S $-0.376422-1.532651-2.730768$ $\begin{array}{llll}\text { O } & 1.049908 & -1.894334 & -2.833917\end{array}$ $\begin{array}{llll}0 & -1.180107 & -1.576930 & -3.967427\end{array}$ o $-0.594602-0.235746-1.959682$ C $-0.797762-3.927062 \quad 2.027249$ $\begin{array}{llll}\text { C }-0.250563 & -5.327065 & 2.344276\end{array}$ C $-1.936876-3.564704 \quad 2.992637$ н $0.019240-3.2044262 .181692$ H $0.019240-3.2044262 .181692$ $\begin{array}{llll}\mathrm{H} & 0.570046 & -5.605763 & 1.666985\end{array}$ $\begin{array}{llll}\mathrm{H}-1.039646 & -6.088882 & 2.24630\end{array}$ H $0.124347-5.3648553 .378865$ $\mathrm{H}-2.773745-4.2737642 .892721$ $\begin{array}{llll}H & -2.326703 & -2.555295 & 2.796575\end{array}$ $\mathrm{H}-1.583483-3.607068-4.034827$ H $-2.470356-3.491691-3.128801$ H $-3.507868-5.188029-1.581865$ H $-2.731918-5.355713 \quad 0.75120$ C $3.2589751 .667593-1.230269$ $\begin{array}{llll}\text { C } 3.155138 & 1.797696 & 1.224332\end{array}$ C $4.049746 \quad 2.822911-1.228616$ C $3.955264 \quad 2.944964 \quad 1.174204$ C $4.404917 \quad 3.458372 \quad-0.041293$ $\begin{array}{llll}C & 2.878532 & 1.012521 & -2.545092\end{array}$ C $1.999576 \quad 1.940025-3.402553$ C 4.1266480 .5 $\begin{array}{lll}C & 1.126648 & 0.564343-3.348833\end{array}$ $\begin{array}{llll}\text { H } 2.283797 & 0.112623 & -2.341129\end{array}$ $1.0868932 .232091-2.8$ $1.6948202 .855688-3.689238$ H $1.6948201 .414943-4.322975$ H $4.797833-0.065781-2.74184$ $\begin{array}{lllll}\mathrm{H} & 4.708201 & 1.433167 & -3.702347\end{array}$ $\begin{array}{llll}\text { H } 3.806471 & -0.015964 & -4.23004\end{array}$ $\begin{array}{llll}\text { C } 2.680789 & 1.247259 & 2.557668\end{array}$ C $3.801196 \quad 0.453326 \quad 3.27139$ C 2.1294992 .3463773 .492218 H 4.6623191 .1093293 .488347 H $4.162820 \quad-0.383613 \quad 2.652493$ H $3.431060 \quad 0.042422 \quad 4.226657$ H $2.930812 \quad 3.015147 \quad 3.848475$ н 1.3724382 .9613012 .978797 $4.3931063 .230774-2.182262$ $\begin{array}{lllll}H & 4.393106 & 3.230774 & -2.182262\end{array}$ H $5.0266264 .356287-0.061842$ C -0.222088 1.930231 2.0 .1921047 C $-0.2220881 .930231-0.191047$ $\mathrm{H}-0.7068861 .998417-1.175011$ $\begin{array}{llll}\text { H } & 0.778384 & 2.383277 & -0.193312\end{array}$ $\begin{array}{lllll}\text { H } & -0.849004 & 2.386011 & 0.587345\end{array}$ $\begin{array}{lllll}C & -0.880636 & 0.091867 & 1.834732\end{array}$ $\begin{array}{llll}\text { C }-1.843815 & -0.071217 & 0.82621\end{array}$ н $-0.723972 \quad 1.064654 \quad 2.305747$ H $-0.539377 \quad-0.768933 \quad 2.41650$ H $-2.442458 \quad 0.775386 \quad 0.484272$ н $-2.258933-1.057544 \quad 0.597884$ H

$\mathrm{Ni}-0.008066 \quad 0.002892-0.002306$ $\mathrm{Ni}-0.0080660 .002892-0.002306$ C $1.9938610 .005479-0.009702$ c $2.8281253-1.1777940 .070532$ C $2.541253-1.1777940 .070532$ c $4.002856-1.4471150 .210440$ H $4.591318-0.5240730 .147751$ H $4.345611-2.142252-0.570897$ H $4.202345-1.9323681 .181229$ $\begin{array}{llll}\text { C } 1.601931-2.305441 & -0.044839\end{array}$ $\begin{array}{llll}\text { C } 2.120147-3.668979 & -0.348983\end{array}$ H $2.738097-3.625803-1.260068$ H $2.751448-4.0419280 .473171$ $\begin{array}{llll}\mathrm{H} & 1.301065 & -4.374459 & -0.528114\end{array}$ N $0.345064-1.9820250 .031081$ C $-0.670003-2.919813-0.332906$ C $-1.207344-2.748644-1.622441$ C $-1.155751-3.9224960 .521623$ C $-2.228037-3.571686-2.077921$ $\begin{array}{llll}C & -2.228037 & -3.571686 & -2.077921\end{array}$ C $-2.180990-4.7426710 .029076$

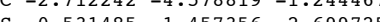
o $0.897521-1.767461-2.846826$

o $-1.359231-1.446702-3.910867$ $\begin{array}{lllll}0 & -0.770033 & -0.186824 & -1.869667\end{array}$ C $-0.630821-4.136401 \quad 1.930418$ $\begin{array}{llll}\text { C }-0.039214 & -5.542925 & 2.114729\end{array}$ $\begin{array}{llll}\text { C }-1.728911 & -3.884575 & 2.975324\end{array}$ H $0.167541 \quad-3.4037692 .116761$ H $0.749987-5.759082 \quad 1.379825$ H $0.392547 \quad-5.637078 \quad 3.123014$ H $-0.815203-6.317298 \quad 2.009537$ H $-2.161173-2.878932 \quad 2.873369$ H $-1.309143-3.968577 \quad 3.988882$ H $-2.543252-4.619694 \quad 2.875244$ H $-2.621246-3.411205-3.081715$ $\mathrm{H}-3.508740-5.238116-1.590449$ $\begin{array}{lllll}\text { H } & -2.573858 & -5.532116 & 0.670664\end{array}$ C $3.0847711 .800515-1.238448$ $\begin{array}{llll}\text { C } 3.313841 & 1.704520 & 1.208793\end{array}$ C $3.8421332 .974193-1.239138$ $\begin{array}{llll}\text { C } 4.068164 & 2.884486 & 1.154292\end{array}$ C $4.3320243 .520106-0.054640$ C $2.6075651 .191296-2.541006$ $\begin{array}{llll}\text { C } 2.160384 & 2.233672 & -3.569485\end{array}$ $\begin{array}{lllll}\text { C } 3.705898 & 0.285571 & -3.159798\end{array}$ H $1.7498220 .539047 \quad-2.326433$ H $1.446337 \quad 2.953881 \quad-3.136646$ H $1.665536 \quad 1.725703-4.413364$ $\begin{array}{lllll}\text { H } & 1.665536 & 1.725703 & -4.413364 \\ \text { H } & .013851 & 2.799519 & -3.980217\end{array}$ H $3.0138512 .799519-3.980217$ $\begin{array}{llll}\text { H } & 4.014641 & -0.507596 & -2.461527\end{array}$ H $3.316433-0.199338-4.069663$ $\begin{array}{lllll}\text { H } & 4.597536 & 0.883010 & -3.419417\end{array}$ C 3.0377201 .0683382 .560203 $\begin{array}{llll}\text { C } 2.188335 & 1.998431 & 3.453914\end{array}$ $\begin{array}{llll}\text { C } 4.335374 & 0.674190 & 3.29466\end{array}$ $\begin{array}{lllll}\text { H } 2.456421 & 0.149277 & 2.404515\end{array}$ H $1.2835802 .341982 \quad 2.928264$ H $2.762008 \quad 2.894824 \quad 3.746315$ H $1.883291 \quad 1.467246 \quad 4.370667$ H $4.970818 \quad 0.018367 \quad 2.678115$ H $4.929651 \quad 1.566144 \quad 3.556604$ H $4.0901590 .143695 \quad 4.230105$ H $4.050002 \quad 3.473098 \quad-2.187690$ H $4.917915 \quad 4.441924-0.075332$ H $4.450430 \quad 3.313620 \quad 2.083371$ C $-0.155532 \quad 1.930881 \quad-0.275698$ $\begin{array}{llll}\text { C } & -0.155532 & 1.930881 & -0.275698 \\ \text { H } & 0.595451 & 2.430582 & 0.350883\end{array}$ $\begin{array}{llll}\text { H } & 0.595451 & 2.430582 & 0.350883 \\ \text { H } & 0.061130 & 2.030629 & -1.345908\end{array}$ $\begin{array}{llll}\mathrm{H} & 0.061130 & 2.030629 & -1.345908 \\ \mathrm{H} & -1.172281 & 2.280421 & -0.052945\end{array}$ $\begin{array}{lrrr}\text { H } & -1.172281 & 2.280421 & -0.052945 \\ \text { C } & -1.739030 & -0.092711 & 0.966377\end{array}$ $\begin{array}{llll}\text { C } & -1.739030 & -0.092711 & 0.966377\end{array}$ $\begin{array}{llll}\mathrm{C} & -0.713354 & 0.291231 & 1.867950 \\ \mathrm{H} & -2.101490 & -1.123717 & 0.936990\end{array}$ $\begin{array}{llll}\mathrm{H} & -2.101490 & -1.123717 & 0.936990 \\ \mathrm{H} & -2.436790 & 0.665258 & 0.608972\end{array}$ H -0.6359801 .3404292 .164208$ C $-0.095574-0.602587 \quad 2.788169$ N $0.449462-1.296009 \quad 3.556518$ 76

Ni $0.058357-0.091281-0.265463$ N $1.927237-0.048534 \quad 0.018660$ C $2.743209 \quad 1.131183 \quad 0.012387$ C $2.525683-1.229426 \quad 0.087708$ $\begin{array}{llll}C & 2.525683 & -1.229426 & 0.087708\end{array}$ $\begin{array}{llll}C & 3.996426 & -1.434655 & 0.246497\end{array}$ $\begin{array}{llll}\mathrm{H} & 4.505817 & -0.506571 & 0.535171 \\ \mathrm{H} & 4.440248 & -1.785421 & -0.701171\end{array}$ $\begin{array}{llll}\mathrm{H} & 4.440248 & -1.785421 & -0.701171 \\ \mathrm{H} & 4.200286 & -2.208305 & 1.003246\end{array}$ $\begin{array}{llll}\text { H } & 4.200286 & -2.208305 & 1.003246 \\ \text { C } 1.632866 & -2.361313 & -0.062793\end{array}$ $\begin{array}{llll}\text { C } & 1.632866 & -2.361313 & -0.062793 \\ \text { C } & 2.160482 & -3.745608 & -0.230138\end{array}$ $\begin{array}{llll}\text { C } & 2.160482 & -3.745608 & -0.230138 \\ \text { H } & 2.914450 & -3.761768 & -1.032172\end{array}$ $\begin{array}{llll}\text { H } 2.644239 & -4.099268 & 0.695186\end{array}$ H $1.357575-4.440421-0.50526$ N $0.367236-2.028294-0.109830$ C $-0.643580-2.956155-0.479459$ C $-1.082975-2.900898-1.815001$ $\begin{array}{llll}\text { C }-1.242180 & -3.826584 & 0.444519\end{array}$ C $-2.113353-3.728949-2.244391$ C $-2.271872-4.655923-0.020466$ $\begin{array}{llll}C & -2.271872 & -4.655923 & -0.020466\end{array}$ $\begin{array}{llll}\text { C } & -2.703095 & -4.615956 & -1.343242\end{array}$ $\begin{array}{llll}\text { S }-0.272646 & -1.746540 & -2.971717\end{array}$ $0.459744-0.404494-2.292237$ 0 $0-1.042771-1.796911-4.212630$ $\begin{array}{lllll}C & -0.816210 & -3.868411 & 1.902098\end{array}$ $\begin{array}{llll}C & -0.321145 & -5.260838 & 2.319336\end{array}$ C $-1.950323-3.399621 \quad 2.825888$ H $0.017514-3.160098 \quad 2.023669$ H $0.491493 \quad-5.615063 \quad 1.668248$ H $0.049395 \quad-5.237294 \quad 3.356099$ H $-1.134966-6.0013832 .272396$ H $-2.281729-2.3844852 .562849$ H $-1.612074-3.392820 \quad 3.874114$ н $-2.818325 \quad-4.074351 \quad 2.756148$ H $-2.431830-3.666814-3.285602$ H $-3.500264-5.281829-1.675770$ H $-2.744934-5.3521650 .673906$ 
$\begin{array}{llll}\text { C } 4.266736 & 3.457343 & -0.002068\end{array}$ $\begin{array}{llll}\text { C } 3.333017 & 0.681553 & -2.449561\end{array}$ $\begin{array}{llll}\text { C } 2.445248 & 1.402907 & -3.488571\end{array}$ $\begin{array}{lllll}\text { C } 4.718875 & 0.374083 & -3.049714\end{array}$ H $2.837397-0.276483-2.240982$ H $1.418296 \quad 1.529117-3.113205$ $\begin{array}{lllll}\text { H } & 2.861503 & 2.393944 & -3.740689\end{array}$ H $2.391077 \quad 0.797530 \quad-4.408734$ H $5.402354-0.064032-2.302608$ H $5.1980281 .284116-3.449437$ H $4.609984-0.338273-3.884389$ $\begin{array}{llll}\text { H } & 4.609984 & -0.338273 & -3.88438 \\ \text { C } & 2.015003 & 1.539805 & 2.418269\end{array}$ C $2.015003 \quad 1.5398052 .418269$ $\begin{array}{llll}C & 1.336982 & 2.734929 & 3.102609\end{array}$ $\begin{array}{llll}\text { C } & 2.917141 & 0.796045 & 3.420277 \\ \text { H } & 1.220479 & 0.844973 & 2.104429\end{array}$ $\begin{array}{cccc}\text { H } & 1.220479 & 0.844973 & 2.104429 \\ \text { H } & 3.356937 & -0.108991 & 2.970935\end{array}$ $\begin{array}{llll}\text { H } & 3.356937 & -0.108991 & 2.970935 \\ \text { H } & 3.744423 & 1.446383 & 3.754272\end{array}$ $\begin{array}{lllll}\mathrm{H} & 3.744423 & 1.446383 & 3.754272 \\ \mathrm{H} & 2.334855 & 0.492226 & 4.307002\end{array}$ H $2.3348550 .492226 \quad 4.307002$ $\begin{array}{llll}\text { H } & 0.743689 & 3.324433 & 2.384771 \\ \text { H } & 2.073038 & 3.408504 & 3.573258\end{array}$ H $2.073038 \quad 3.408504 \quad 3.573258$ H $0.6645442 .376463 \quad 3.900110$ H $4.7250712 .966327 \quad-2.045703$ H $4.868570 \quad 4.369455 \quad-0.007798$ H $3.615165 \quad 3.723580 \quad 2.031796$ C $-0.161041 \quad 1.839269-0.475277$ H $0.775665 \quad 2.250993-0.870308$ $\begin{array}{llll}\mathrm{H} & -0.983475 & 1.941986 & -1.196422\end{array}$ $\begin{array}{lllll}\mathrm{H} & -0.418532 & 2.282976 & 0.496083\end{array}$ $\mathrm{N}-1.679768-0.1372830 .300982$ $\begin{array}{lllll}C & -2.825223 & -0.241419 & 0.491469\end{array}$ $\begin{array}{llll}C & -4.210204 & -0.344460 & 0.735522\end{array}$ $\begin{array}{llll}\mathrm{H} & -4.692611 & 0.552586 & 1.139792\end{array}$ $\begin{array}{lllll}C & -4.913775 & -1.463504 & 0.482321\end{array}$ $\begin{array}{lllll}\text { H } & -4.434999 & -2.351741 & 0.062398\end{array}$ 76

$17 \mathrm{R}=\mathrm{Me} \mathrm{AC} / \mathrm{N}-\mathrm{C}$

Ni $0.020718-0.041956-0.151179$ N $1.982542-0.008666-0.048326$ C $2.781242 \quad 1.175357 \quad 0.004328$ $\begin{array}{lll}\text { C } 2.538626 & -1.197893-0.011737\end{array}$ $\begin{array}{llll}\text { C } 4.006326 & -1.461277 & 0.048200\end{array}$ H $4.569495-0.5439760 .261980$ H $4.351585-1.858220-0.922184$ $\begin{array}{llll}\text { H } & 4.351585 & -1.858220 & -0.922184 \\ \text { H } & 4.244312 & -2.220014 & 0.809564\end{array}$ $\begin{array}{llll}\text { H } & 4.244312 & -2.220014 & 0.809564 \\ \text { C } & 1.585133 & -2.285444 & -0.121069\end{array}$ $\begin{array}{llll}\text { C } 1.585133 & -2.285444 & -0.121069 \\ \text { C } 2.050541 & -3.677144 & -0.384013\end{array}$ $\begin{array}{llll}\text { C } & 2.050541 & -3.677144 & -0.384013 \\ \text { H } 2.667358 & -3.689442 & -1.295985\end{array}$ $\begin{array}{llll}\text { H } 2.667358 & -3.689442 & -1.295985 \\ \text { H } 2.667270 & -4.044213 & 0.452774\end{array}$ $\begin{array}{lllll}\text { H } 2.667270 & -4.044213 & 0.452774\end{array}$ H $1.208232-4.361928-0.537519$ N $0.320957-1.914709-0.015475$ C $-0.695351-2.838243-0.428020$ $\begin{array}{llll}\text { C }-1.026508 & -2.827944 & -1.793167\end{array}$ $\begin{array}{llll}\text { C }-1.370667 & -3.666310 & 0.477214\end{array}$ $\begin{array}{llll}\text { C }-2.024190 & -3.668398 & -2.272261\end{array}$ $\begin{array}{llll}\text { C }-2.361813 & -4.512677 & -0.037434\end{array}$ C $-2.685173-4.521432-1.389734$ S - $0.203724-1.657185-2.923633$ o $1.233544-1.997165-2.882344$ $\begin{array}{llll}0 & 1.233544 & -1.997165 & -2.882344 \\ 0 & -0.895946 & -1.836199 & -4.222685\end{array}$ $\begin{array}{llll}0 & -0.895946 & -1.836199 & -4.222685 \\ 0 & -0.505617 & -0.322495 & -2.274341\end{array}$ $\begin{array}{llll}0 & -0.505617 & -0.322495 & -2.274341 \\ \text { C }-1.047858 & -3.668366 & 1.960333\end{array}$ $\begin{array}{llll}\text { C }-1.047858 & -3.668366 & 1.960333 \\ \text { C }-0.349097 & -4.971184 & 2.380219\end{array}$ $\begin{array}{llll}\text { C } & -0.349097 & -4.971184 & 2.380219 \\ \text { C } & -2.299811 & -3.435019 & 2.818049\end{array}$ $\begin{array}{llll}C & -2.299811 & -3.435019 & 2.818049 \\ \mathrm{H}-0.351545 & -2.834350 & 2.143816\end{array}$ $\begin{array}{llll}\mathrm{H} & -0.351545 & -2.834350 & 2.143816\end{array}$ H $0.574883 \quad-5.138871 \quad 1.808205$ H $-0.094089 \quad-4.941914 \quad 3.451290$ н $-1.009899-5.8365332 .211638$ н $-2.837697-2.5281732 .505766$ H $-2.019496-3.330134 \quad 3.877550$ H $-2.997009-4.283856 \quad 2.741572$ н $-2.269279-3.629071-3.333883$ H $-3.466162-5.187627-1.758314$ $\begin{array}{lllll}\text { H }-2.900365 & -5.173889 & 0.642267\end{array}$ $\begin{array}{llll}\text { C } 3.352509 & 1.671821 & -1.182994\end{array}$ C 2.9162961 .8512321 .233168 $\begin{array}{llll}\text { C } & 4.9105385 & 2.849855 & -1.103749\end{array}$ $\begin{array}{llll}\text { C } & 4.105385 & 2.849855 & -1.103749\end{array}$ $\begin{array}{llll}\text { C } 3.680209 & 3.023434 & 1.260986 \\ \text { C } 4.279972 & 3.521037 & 0.105090\end{array}$ $\begin{array}{llll}\text { C } 3.147345 & 0.987340 & -2.522185\end{array}$ $\begin{array}{llll}\text { C } 2.155904 & 1.782087 & -3.389615\end{array}$ C $4.471408 \quad 0.766229-3.272068$ H $2.6892240 .002026-2.354418$ $\begin{array}{lllll}\mathrm{H} & 1.175374 & 1.862783 & -2.895085\end{array}$ H $2.5411412 .796235-3.598240$ H $2.000204 \quad 1.262290-4.349618$ H $5.216952 \quad 0.254570 \quad-2.639726$ H $4.913026 \quad 1.718819-3.611512$ H $4.290067 \quad 0.146101 \quad-4.165639$ $\begin{array}{llll}\text { C } 2.283808 & 1.311786 & 2.502572\end{array}$ $\begin{array}{lllll}\text { C } 1.595269 & 1.311786 & 2.502572\end{array}$ C $3.313788 \quad 0.552895 \quad 3.365953$ c 3.3137880 .5528953 .365953 H 0.8761392 .9752992 .726339 H 1.0483671 .9432934 .175667 H $3.772082-0.280107 \quad 2.808802$ $\begin{array}{lllll}\text { H } 2.828061 & 0.140058 & 4.266673\end{array}$ H $4.5698293 .245058-2.010550$ H $4.884190 \quad 4.431068 \quad 0.147626$ H $3.810782 \quad 3.555734 \quad 2.206076$ C $-1.846104 \quad-0.223484 \quad 0.430802$ н $-2.364833-0.699355-0.412178$ H $-1.814773-0.865183 \quad 1.320276$ $\begin{array}{lllll}\mathrm{H} & -2.279181 & 0.753862 & 0.673331\end{array}$ $\begin{array}{lllll}N & -0.181817 & 1.747444 & -0.138036\end{array}$ C $-0.265767 \quad 2.894566-0.328352$ $\begin{array}{llll}\text { C }-0.265767 & 2.894566 & -0.328352\end{array}$ $\begin{array}{llll}C & -0.406326 & 4.280783 & -0.551723\end{array}$ $\begin{array}{llll}\text { C } & 0.644599 & 5.111039 & -0.689137\end{array}$ $\begin{array}{llll}\mathrm{H} & -1.436974 & 4.647034 & -0.62217\end{array}$ H $1.671708 \quad 4.742990-0.626226$ $\begin{array}{lllll}\text { H } & 0.481431 & 6.175037 & -0.868169 \\ \text { H } & 1.505025 & 0.595014 & 2.199756\end{array}$ 82

$17 \mathrm{R}=\mathrm{CHCNEt}$ ET/pi

Ni $0.014913-0.013835 \quad 0.001558$ $\begin{array}{llll}\mathrm{N} 2 & 2.039584 & -0.018328 & 0.015191\end{array}$ $\begin{array}{llll}\text { C } 2.880943 & 1.146096 & -0.015682\end{array}$ $\begin{array}{llll}\text { C } 2.575191 & -1.206170 & 0.121852\end{array}$ C $4.033901-1.501146 \quad 0.242250$ $\begin{array}{llll}\text { H } 4.636856 & -0.588678 & 0.164379\end{array}$ н $4.349542-2.206368-0.542085$ H $4.245229-1.9863791 .210326$ C $1.245229-1.9863791 .210326$ C $1.615470-2.3159070 .075722$ $\begin{array}{llll}\text { C } 2.101669 & -3.735463 & -0.151371\end{array}$ $2.6768779-3.055614-1.098000$ H $2.768779-4.0564090 .665926$ H $1.254578-4.429103-0.235267$ $\mathrm{N} 0.366940-1.9617620 .154971$ $\begin{array}{llll}\text { C }-0.685491 & -2.890655 & -0.102242\end{array}$ $\begin{array}{llll}\text { C }-1.223343 & -2.859802 & -1.398435\end{array}$ $\begin{array}{llll}\text { C }-1.231289 & -3.723668 & 0.886727\end{array}$ C $-2.308276-3.662991-1.732201$ $\begin{array}{llll}\text { C }-2.317284 & -4.531303 & 0.526028\end{array}$ C $-2.862522-4.507070-0.772782$ S $-0.489484-1.739480-2.623492$ o $0.904272-2.180033-2.779965$ o $-1.361077-1.763202-3.802130$ $\begin{array}{llll}\text { O } & -0.598160 & -0.384443 & -1.917438 \\ \text { C }-0.704873 & -3.752816 & 2.309577\end{array}$ C $-0.714628-5.158272 \quad 2.679446$ $\begin{array}{llll}C & -0.114628 & -5.158272 & 2.679446\end{array}$ $\begin{array}{llll}C & -1.782166 & -3.349481 & 3.32514\end{array}$ H $0.119592-3.0271472 .380717$ H $0.671233-5.4655301 .971208$ H $0.314694-5.1284933 .694992$ H $-0.906952 \quad-5.925363 \quad 2.664614$ H $-2.192284-2.353272 \quad 3.10374$ H $-1.360507 \quad-3.332988 \quad 4.342283$ H $-2.617583-4.067195 \quad 3.320064$ H $-2.706445-3.603417-2.748072$ н $-3.717808-5.140014-1.019210$ $\begin{array}{llll}\mathrm{H} & -2.763532 & -5.190540 & 1.273504\end{array}$ $\begin{array}{llll}\text { C } 3.167509 & 1.708060 & -1.277657\end{array}$ $\begin{array}{llll}\text { C } 3.337275 & 1.737424 & 1.179087\end{array}$ C $3.901632 \quad 2.896578-1.320249$ $\begin{array}{llll}\text { C } 4.064108 & 2.931182 & 1.078917\end{array}$ $\begin{array}{llll}\text { C } 4.339339 & 3.517896 & -0.154073\end{array}$ $\begin{array}{llll}\text { C } & 2.349339 & 3.517896 & -0.154073 \\ \text { C } & 2.319489 & 1.018411 & -2.564763\end{array}$ $\begin{array}{llll}\text { C } 2.319489 & 1.996273 & -3.662159\end{array}$ $\begin{array}{llll}\text { C } 3.883065 & 0.124147 & -3.103287\end{array}$ $\begin{array}{llll}\text { C } & 3.883065 & 0.124147 & -3.103287 \\ \text { H } & 1.893102 & 0.361229 & -2.347252\end{array}$ $\begin{array}{llll}\text { H } & 1.893102 & 0.361229 & -2.347252 \\ \text { H } & 4.190083 & -0.634314 & -2.367342\end{array}$ $\begin{array}{llll}\text { H } & 4.190083 & -0.634314 & -2.367342 \\ \text { H } & 4.769569 & 0.734393 & -3.351338\end{array}$ $\begin{array}{lllll}\text { H } & 4.769569 & 0.734393 & -3.351338 \\ \text { H } & 3.546926 & -0.404121 & -4.010665\end{array}$ $\begin{array}{lllll}\text { H } & 3.546926 & -0.404121 & -4.010665 \\ \text { H } & 1.588163 & 2.733507 & -3.293770\end{array}$ H $3.1810412 .548944-4.074103$ H $1.857955 \quad 1.437592 \quad-4.492586$ C $3.089839 \quad 1.129333 \quad 2.552458$ $\begin{array}{llll}\text { C } 2.416580 & 2.111914 & 3.527018\end{array}$ C $4.412973 \quad 0.609773 \quad 3.186886$ H $2.414353 \quad 0.269662 \quad 2.427113$ H $4.936275-0.109306 \quad 2.537890$ $4.9362750 .129306 \quad 2.537890$

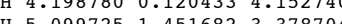
\begin{tabular}{l}
1.099251 .4516823 .378704 \\
\hline
\end{tabular} $\begin{array}{ll}1.523433 & 2.5784133 .086352\end{array}$ . 2.1335771 .5831464 .453488 $\begin{array}{llll}\text { H } 3.111079 & 2.921858 & 3.809387 \\ \text { H } 4.128584 & 3.348705 & -2.287457\end{array}$ $\begin{array}{lllll}\mathrm{H} & 4.128584 & 3.348705 & -2.287457 \\ \mathrm{H} & 4.897748 & 4.455345 & -0.204468\end{array}$ $\begin{array}{lllll}\mathrm{H} & 4.897748 & 4.455345 & -0.204468 \\ \mathrm{H} & 4.411690 & 3.416276 & 1.993793\end{array}$ $\begin{array}{lllll}\text { H } & 4.411690 & 3.416276 & 1.993793 \\ \text { C } & -0.228970 & 1.923919 & -0.49940\end{array}$ H $0.598585 \quad 1.962723-1.223323$ C $0.0310292 .821896 \quad 0.58528$ N $0.144881 \quad 3.559782 \quad 1.487038$ C $-1.559924 \quad 2.256735-1.183359$ C -1.439124 $3.526134 \quad-2.086013$ H $-2.334496 \quad 2.444705 \quad-0.425532$ H $-1.866228 \quad 1.403372-1.801640$ $+0.7285303 .34$ $\begin{array}{llll}-1 & -0.728530 & 3.346673 & -2.908699\end{array}$ $\begin{array}{llll}\mathrm{H} & -1.093557 & 4.394996 & -1.50174\end{array}$ $\begin{array}{llll}\mathrm{H}-2.421443 & 3.764789 & -2.526249\end{array}$ C -0.6547560 .1600341 .908013$ $\begin{array}{llll}\text { C } & -1.728738 & -0.048089 & 1.031285 \\ \mathrm{H}-0.231784 & -0.674812 & 2.473679\end{array}$

H $-0.450619 \quad 1.154334 \quad 2.310787$ $\begin{array}{llll}\mathrm{H} & -2.379690 & 0.781717 & 0.756347\end{array}$ 82

$17 \mathrm{R}=\mathrm{CHCNEt}$ ET/pi

Ni $0.014913 \quad-0.013835 \quad 0.001558$ $\begin{array}{llll}\mathrm{N} 2 & 2.039584 & -0.018328 & 0.015191\end{array}$ C $2.8809431 .146096-0.015682$ $\begin{array}{llll}\text { C } 2.575191 & -1.206170 & 0.121852\end{array}$ C $4.033901-1.5011460 .242250$ $\begin{array}{llllll}C & \text { C } \\ \text { H } 4.636856 & -0.588678 & 0.242250\end{array}$ $\begin{array}{lllll}\text { H } 4.636856 & -0.588678 & 0.164379\end{array}$ $\begin{array}{llll}\text { H } 4.349542 & -2.206368 & -0.542085\end{array}$ $\begin{array}{llll}\text { H } & 4.245229 & -1.986379 & 1.210326\end{array}$ $\begin{array}{llll}\text { C } & 2.615470 & -2.3159063 & 0.075722 \\ \text { C } & .101669 & -3.735463 & -0.151371\end{array}$ $\begin{array}{llll}\text { C } & 2.101669 & -3.735463 & -0.151371 \\ \text { H } & 2.667240 & -3.765914 & -1.098000\end{array}$ $\begin{array}{llll}\text { H } 2.667240 & -3.765914 & -1.098000\end{array}$ H $2.768779-4.0564090 .665926$ H $1.254578-4.429103-0.235267$ $\begin{array}{llll}\text { N } 0.366940 & -1.961762 & 0.154971\end{array}$ $\begin{array}{llll}\text { C }-0.685491 & -2.890655 & -0.102242\end{array}$ $\begin{array}{llll}\text { C }-1.223343 & -2.859802 & -1.398435\end{array}$ $\begin{array}{llll}\text { C }-1.231289 & -3.723668 & 0.886727\end{array}$ $\begin{array}{llll}C & -2.308276 & -3.662991 & -1.732201\end{array}$ $\begin{array}{llll}\text { C }-2.317284 & -4.531303 & 0.526028\end{array}$ C $-2.862522-4.507070-0.772782$ S $-0.489484-1.739480-2.623492$ o $0.904272-2.180033-2.779965$ $0.904272-2.180033-2.779965$ $0-1.361077-1.763202-3.802130$ $\begin{array}{llll}0 & -0.598160 & -0.384443 & -1.91743\end{array}$ $\begin{array}{llll}C & -0.704873 & -3.752816 & 2.309577\end{array}$ $\begin{array}{llll}C & -0.114628 & -5.158272 & 2.679446\end{array}$ $\begin{array}{lllll}C & -1.782166 & -3.349481 & 3.325141\end{array}$ $\begin{array}{llll}\text { H } & 0.119592 & -3.027147 & 2.380717\end{array}$ H $0.671233-5.465530 \quad 1.971208$ H $0.314694 \quad-5.128493 \quad 3.694992$ $\begin{array}{llll}\text { H } & -0.906952 & -5.925363 & 2.664614\end{array}$ H $-2.192284-2.353272 \quad 3.103744$ H $-1.360507-3.332988 \quad 4.342283$ H $-2.617583-4.067195 \quad 3.320064$ H $-2.706445-3.603417-2.748072$ $\mathrm{H}-3.717808-5.140014-1.019210$ H $-2.763532-5.190540 \quad 1.273504$ C $3.167509 \quad 1.708060-1.277657$ C $3.337275 \quad 1.737424 \quad 1.179087$ C $3.9016322 .896578-1.320249$ $\begin{array}{llll}\text { C } 4.064108 & 2.931182 & 1.078917\end{array}$ C $4.3393393 .517896-0.154073$ $\begin{array}{llll}\text { C } 2.748457 & 1.018411 & -2.564763\end{array}$ C $2.3194891 .996273-3.662159$ C $3.883065 \quad 0.124147-3.103287$ H $1.8931020 .361229 \quad-2.347252$ H $4.190083-0.634314-2.367342$ H $4.7695690 .734393 \quad-3.351338$ H $3.546926-0.404121-4.010665$ H $1.5881632 .733507-3.293770$ H $3.1810412 .548944-4.074103$ H $1.857955 \quad 1.437592 \quad-4.49258$ C 3.0898391 .1293332 .552458 C $2.4165802 .111914 \quad 3.527018$ C $4.4129730 .609773 \quad 3.186886$ $\begin{array}{llll}\text { H } 2.414353 & 0.269662 & 2.427113\end{array}$ H $4.936275 \quad-0.109306 \quad 2.537890$ H $4.1987800 .120433 \quad 4.152740$ $\begin{array}{llll}\text { H } & 4.198780 & 0.120433 & 4.152740 \\ \text { H } & 5.099725 & 1.451682 & 3.378704\end{array}$ $\begin{array}{llll}\text { H } & 1.523433 & 2.578413 & 3.086352\end{array}$ $\begin{array}{llll}\mathrm{H} & 1.523433 & 2.578413 & 3.086352 \\ \mathrm{H} & 2.133577 & 1.583146 & 4.453488\end{array}$ H $3.1110792 .921858 \quad 3.809387$ H $4.1285843 .348705-2.287457$ H $4.897748 \quad 4.455345 \quad-0.204468$ H $4.411690 \quad 3.416276 \quad 1.993793$ C $-0.2289701 .923919-0.499403$ H $0.598585 \quad 1.962723-1.223323$ C $0.031029 \quad 2.821896 \quad 0.585281$ N $0.144881 \quad 3.559782 \quad 1.487038$ C $-1.559924 \quad 2.256735-1.183359$ C $-1.5599242 .256735-1.183359$ $\begin{array}{llll}C & -1.439124 & 3.526134 & -2.086013\end{array}$ $\begin{array}{llll}\mathrm{H} & -2.334496 & 2.444705 & -0.425532\end{array}$ $\mathrm{H}-1.866228-1.403372-1.801640$ $\mathrm{H}-0.7285303 .346673-2.908699$ $\mathrm{H}-1.0935574 .394996-1.501747$ H $-2.421443 \quad 3.764789-2.526249$ C $-0.654756 \quad 0.160034 \quad 1.908013$ C $-1.728738-0.048089 \quad 1.031285$ H $-0.231784-0.674812 \quad 2.473679$ H $-0.450619 \quad 1.154334 \quad 2.310787$ $\begin{array}{lllll}\text { H } & -2.379690 & 0.781717 & 0.756347\end{array}$ H $-2.157773-1.0429710 .879688$ 83

$\mathrm{Ni} 0.000341-0.000496-0.002639$ N $2.038382-0.000496-0.001675$ C $2.8876081 .159871-0.001100$ $\begin{array}{llll}\text { C } 2.561997 & -1.189148 & 0.116527\end{array}$ C $4.016358-1.4846990 .269716$ 
$\begin{array}{llll}\text { C } & 1.605065 & -2.307116 & 0.023150\end{array}$ $\begin{array}{llll}\text { C } 2.102283 & -3.687408 & -0.231381\end{array}$ H $2.715684-3.685152-1.146676$ H $2.732833-4.035700 \quad 0.601807$ H $1.273146-4.387980-0.380708$ N $0.352097-1.968807 \quad 0.080189$ $\begin{array}{llll}\text { C }-0.677632 & -2.908500 & -0.243674\end{array}$ C $-1.209564-2.794945-1.539002$ $\begin{array}{llll}\text { C }-1.186165 & -3.854929 & 0.663072\end{array}$ C $-2.254285-3.611314-1.955305$ $\begin{array}{llll}\text { C }-2.236963 & -4.668095 & 0.219977\end{array}$ $\begin{array}{llll}\text { C } & -2.236963 & -4.668095 & 0.219977 \\ \text { C }-2.777050 & -4.555304 & -1.075866\end{array}$ $\begin{array}{llll}\text { C } & -2.777050 & -4.555304 & -1.075866 \\ \text { S }-0.502592 & -1.577685 & -2.676548\end{array}$ S $-0.502592-1.577685-2.676548$ $\begin{array}{lllll}0 & 0.911127 & -1.941696 & -2.840874\end{array}$ $\begin{array}{llll}0 & -1.357698 & -1.565074 & -3.859619\end{array}$ $0-0.679498-0.268179-1.886108$ $\begin{array}{llll}\text { C } & -0.657106 & -4.016813 & 2.073448\end{array}$ $\begin{array}{llll}\text { C }-0.081826 & -5.455101 & 2.316255\end{array}$ C $-1.730574 \quad-3.699980 \quad 3.122939$ H $0.167344-3.3075812 .223316$ H $0.689782-5.715992 \quad 1.574262$ H $0.365626-5.504964 \quad 3.322946$ H $-0.881300-6.212748 \quad 2.258328$ H $-2.140906-2.6882012 .990321$ H $-1.296076-3.755113 \quad 4.131947$ $\begin{array}{llll}\text { H } & -2.565759 & -4.415864 & 3.064978 \\ \text { H }-2.641221 & -3.486566 & -2.967471\end{array}$ $\begin{array}{llll}\mathrm{H} & -2.641221 & -3.486566 & -2.967471 \\ \mathrm{H} & -3.600203 & -5.204600 & -1.384349\end{array}$ $\begin{array}{llll}\text { H } & -3.600203 & -5.204600 & -1.384349 \\ \text { H } & -2.654094 & -5.411038 & 0.902480\end{array}$ C $3.1079421 .792223-1.245057$ C 3.4343001 .6654601 .195788 C $3.8595512 .970922-1.259283$ C 4.1682882 .8549311 .122216 C $4.3756353 .509632-0.083845$ $\begin{array}{llll}\text { C } 2.635388 & 1.160368 & -2.541912\end{array}$ $\begin{array}{llll}\text { C } 2.205572 & 2.183673-3.604519\end{array}$ $\begin{array}{llll}\text { C } 3.720597 & 0.233320 & -3.115984\end{array}$ H $1.767044 \quad 0.521632 \quad-2.321403$ H $4.006133-0.547594-2.396937$ H $4.625068 \quad 0.809970-3.370134$ H $3.345834-0.266361-4.021651$ H $1.5050612 .928480-3.196414$ H $3.0710902 .723315-4.021693$ $\begin{array}{lllll}\mathrm{H} & 3.071090 & 2.723315 & -4.021693 \\ \mathrm{H} & 1.710092 & 1.659859 & -4.436032\end{array}$ $\begin{array}{llll}\text { H } & 1.710092 & 1.659859 & -4.436032 \\ \text { C } & 3.264196 & 0.998845 & 2.550203\end{array}$ $\begin{array}{llll}\text { C } & 3.264196 & 0.998845 & 2.550203 \\ \text { C } & .501478 & 1.893214 & 3.538118\end{array}$ $\begin{array}{llll}\text { C } & 4.617764 & 0.605730 & 3.166875\end{array}$ H 2.6776360 .0794232 .421321 $\begin{array}{llll}H & 5.218877 & -0.024748 & 2.494318\end{array}$ H $4.450667 \quad 0.052405 \quad 4.104886$ H $5.2150601 .500761 \quad 3.406424$ H $1.5706652 .281038 \quad 3.104542$ H $2.269624 \quad 1.325026 \quad 4.451713$ H $3.110327 \quad 2.766572 \quad 3.822673$ H $4.042006 \quad 3.475457 \quad-2.209259$ H $4.946621 \quad 4.440735-0.112159$ н $4.580889 \quad 3.277494 \quad 2.040867$ C $-0.2557121 .949271-0.484971$ $\begin{array}{lllll}\text { H } & 0.439981 & 1.934984 & -1.335047\end{array}$ $\begin{array}{llll}\mathrm{H} & 0.439981 & 1.934984 & -1.335047\end{array}$ $\begin{array}{llll}C & 0.209304 & 2.853112 & 0.523879 \\ \mathrm{~N} & 0.485281 & 3.609154 & 1.374150\end{array}$ $\begin{array}{llll}\mathrm{N} & -1.668355 & 2.312869 & -0.948876\end{array}$ $\begin{array}{llll}\text { C }-1.668355 & 2.312869 & -0.948876 \\ \text { C }-1.664948 & 3.589982 & -1.832161\end{array}$ $\begin{array}{llll}\text { C }-1.664948 & 3.589982 & -1.832161 \\ \mathrm{H}-2.314246 & 2.495667 & -0.076633\end{array}$ $\begin{array}{llll}\mathrm{H}-2.314246 & 2.495667 & -0.076633 \\ \mathrm{H}-2.074811 & 1.473827 & -1.529140\end{array}$ $\begin{array}{llll}\mathrm{H} & -2.074811 & 1.473827 & -1.529140 \\ \mathrm{H}-1.083183 & 3.419201 & -2.751415\end{array}$ $\begin{array}{llll}\mathrm{H} & -1.083183 & 3.419201 & -2.751415 \\ \mathrm{H} & -1.229000 & 4.443953 & -1.289847\end{array}$ $\begin{array}{llll}\mathrm{H} & -1.229000 & 4.443953 & -1.289847 \\ \mathrm{H} & -2.696924 & 3.845777 & -2.120630\end{array}$ $\begin{array}{llll}\text { H } & -2.696924 & 3.845777 & -2.120630\end{array}$ $\begin{array}{llll}\text { C } & -0.589104 & 0.281226 & 1.922525\end{array}$ $\begin{array}{llll}C & -1.667543 & -0.115233 & 1.092183\end{array}$ $\begin{array}{llll}\text { H } & -0.497986 & 1.331844 & 2.215389\end{array}$ $\begin{array}{lllll}\mathrm{H} & -2.404657 & 0.632205 & 0.803544\end{array}$ $\mathrm{H}-2.015570-1.151041 \quad 1.079761$ $\begin{array}{llll}\text { N } & 0.712446 & -1.297877 & 3.513723\end{array}$ 83

$7 \mathrm{R}=\mathrm{CHCNE}$ AC $/ \mathrm{N}$

Ni $0.000508-0.008206-0.002325$ N $1.911455-0.0051450 .000239$ $\begin{array}{llll}\text { C } 2.758337 & 1.157608 & 0.000763\end{array}$ $\begin{array}{llll}\text { C } 2.472102-1.187541-0.185839 & 0.37930\end{array}$ C $3.932002-1.428622-0.371930$ H $4.529345-0.543686-0.121070$ H $4.130465-1.693612-1.424952$ $\begin{array}{llll}\text { H } & 4.267842 & -2.279038 & 0.241622\end{array}$ $\begin{array}{llll}\text { C } & 1.531921 & -2.291732 & -0.270219\end{array}$ $\begin{array}{llll}\text { C } 1.975787 & -3.669587 & -0.618510\end{array}$ H $2.574646-3.645666-1.541009$ H $2.599478 \quad-4.092757 \quad 0.186315$ H $1.114088-4.326108-0.790526$ N $0.292558-1.933712-0.055457$ C $-0.797185-2.792595-0.370970$ C $-0.797185-2.792595-0.370970$ $\begin{array}{llll}C & -1.323751 & -2.662408 & -1.667168\end{array}$ C $-1.387976-3.6375920 .577296$ C $-2.447117-3.392579-2.035194$ $\begin{array}{llll}\text { C }-2.515610 & -4.366081 & 0.175321 \\ \text { C }-3.040850 & -4.250215 & -1.108248\end{array}$
S $-0.541164-1.502856-2.838124$ o $0.849465-1.976983-2.990233$ $0-1.386176-1.517920-4.032377$ $\begin{array}{llll}0 & -0.621624 & -0.181116 & -2.096332\end{array}$ $\begin{array}{llll}\text { C }-0.830215 & -3.773842 & 1.982368\end{array}$ $\begin{array}{llll}\text { C }-0.289486 & -5.189740 & 2.233560\end{array}$ C $-1.863980-3.393984 \quad 3.051311$ H $0.009145-3.066603 \quad 2.071862$ H $0.463323-5.474712 \quad 1.483965$ H $0.172449-5.2504833 .231295$ $\begin{array}{llll}H & 0.172449 & -5.250483 & 3.231295\end{array}$ $\mathrm{H}-1.101676 \quad-5.932772 \quad 2.191454$ H $-2.218661-2.361974 \quad 2.915432$ $\mathrm{H}-1.417208-3.4712904 .054780$ $\mathrm{H}-2.735263-4.0669993 .019746$ $\mathrm{H}-2.840470-3.270376-3.044815$ $\mathrm{H}-3.919858-4.830867-1.390089$ $\begin{array}{lllll}\text { H } & -2.993317 & -5.039999 & 0.88804\end{array}$ $\begin{array}{llll}\text { C } 3.186609 & 1.697469 & -1.228814\end{array}$ $\begin{array}{llll}\text { C } 3.094737 & 1.746689 & 1.237071\end{array}$ C $3.9767162 .850045-1.188493$ C 3.8936992 .8927401 .218286 C $4.3340963 .446823 \quad 0.020169$ $\begin{array}{llll}\text { C } 2.804536 & 1.101894 & -2.573527\end{array}$ $\begin{array}{llll}\text { C } 1.860490 & 2.039262 & -3.341781\end{array}$ $\begin{array}{llll}\text { C } 4.041504 & 0.778704 & -3.426354\end{array}$ $\begin{array}{lllll}\text { H } 2.253902 & 0.164677 & -2.411722\end{array}$ $\begin{array}{lllll}\text { H } 2.253902 & 0.164677 & -2.411722\end{array}$ $\begin{array}{lllll}\text { H } & 4.767893 & 0.159676 & -2.878494\end{array}$ $\begin{array}{lllll}\text { H } & 4.559787 & 1.696875 & -3.746078\end{array}$ $\begin{array}{lllll}\text { H } & 3.733484 & 0.233484 & -4.331296\end{array}$ H $0.9428592 .231329-2.770505$ H $2.3485603 .003537-3.557497$ $\begin{array}{lllll}\text { H } 1.568023 & 1.574491 & -4.295374\end{array}$ C $2.639281 \quad 1.139934 \quad 2.55412$ C $2.441378 \quad 2.176401 \quad 3.663780$ $\begin{array}{llll}\text { C } 3.616809 & 0.049618 & 3.026057\end{array}$ н $1.6640910 .659572 \quad 2.370748$ H $3.706375-0.7689362 .298108$ H $3.271655-0.379040 \quad 3.980103$ H $4.6208310 .476922 \quad 3.183319$ H $1.7913372 .999462 \quad 3.336873$ H $1.960908 \quad 1.696279 \quad 4.529233$ H $3.4033592 .588004 \quad 4.008687$ H $4.317323 \quad 3.2924$ $\begin{array}{lllll}\text { H } & 4.317323 & 3.292419 & -2.126275\end{array}$ $\begin{array}{lllll}\text { H } & 4.951541 & 4.348664 & 0.025274\end{array}$ H 4.1654743 .3695592 .161155 C $-0.216887 \quad 1.9791890 .096800$ H $0.7756072 .370100-0.178760$ $\begin{array}{llll}c & -0.495542 & 2.313665 & 1.465637\end{array}$ $\mathrm{N}-0.7753392 .573994 \quad 2.572763$ C $-1.2905092 .503333-0.864410$ C $-1.270459 \quad 4.033539-0.955300$ H $-2.283116 \quad 2.163057-0.532051$ н $-1.121766 \quad 2.056704-1.853799$ H $-0.296255 \quad 4.394619-1.320764$ H $-1.455016 \quad 4.491976 \quad 0.028662$ H $-2.047172 \quad 4.384136-1.652231$ $\begin{array}{lllll}\mathrm{N} & -1.697989 & -0.167340 & 0.648481\end{array}$ C $-2.803703-0.317135 \quad 0.980245$ C $-4.138955-0.5520801 .360358$ C $-4.138955-0.5520801 .360358$ $\begin{array}{llll}C & -4.828179 & 0.275894 & 2.183875\end{array}$ $\begin{array}{lllll}\mathrm{H} & -4.597070 & -1.447616 & 0.93259\end{array}$ $\begin{array}{llll}\text { H } & -5.866711 & 0.056534 & 2.437642 \\ \mathrm{H} & -4.363295 & 1.169234 & 2.608232\end{array}$ 81 $17 \mathrm{R}=\mathrm{n}-\mathrm{Pr} \mathrm{ET} / \mathrm{p}$

Ni $-0.016296 \quad 0.001176 \quad-0.072454$ N $1.972306 \quad 0.002076 \quad-0.009342$ $\begin{array}{llll}\text { C } 2.795807 & 1.176044 & 0.010989\end{array}$ $\begin{array}{llll}\text { C } 2.538624 & -1.176915 & -0.078122\end{array}$ C $4.008798-1.431289-0.140592$ $\begin{array}{lllll}\text { H } 4.586414 & -0.524132 & 0.075477\end{array}$ H $4.279047-1.780675-1.15178$ H $4.303381-2.226608 \quad 0.561805$ $\begin{array}{llll}\text { C } 1.602364 & -2.299908 & -0.188913\end{array}$ C $2.101266-3.667700-0.509006$ C $2.101266-3.667700-0.509006$ H $2.716689-3.631590-1.420764$ $\begin{array}{llll}\text { H } 2.727527 & -4.056278 & 0.310680\end{array}$ H $1.269186-4.359713-0.687058$ $\mathrm{N} 0.349239-1.970982-0.053242$ C $-0.679734-2.878842-0.434162$ c $-1.133704-2.748576-1.758454$ $\begin{array}{llll}C & -1.275846 & -3.786646 & 0.451132\end{array}$ $\begin{array}{llll}\text { C }-2.179341 & -3.538439 & -2.218682\end{array}$ C $-2.324070-4.574777-0.042940$ C $-2.771422-4.460387-1.354662$ S $-0.356006-1.510596-2.846614$ O $1.063078-1.899229-2.939359$ $0-1.135852-1.515700-4.080272$ $0-0.556272-0.215346-2.066304$ C $-0.825675-3.919690 \quad 1.894680$ C $-0.290159-5.327270 \quad 2.196563$ C $-1.952871-3.5514972 .871354$ C $0.000408-3.2074452 .049184$ H $0.000408-3.2074452 .049184$ $\mathrm{H}-1.084934 \quad-6.081620 \quad 2.087510$ $\begin{array}{lllll}\text { H } & -1.084934 & -6.081620 & 2.087510 \\ \text { H } & 0.082672 & -5.379876 & 3.231361\end{array}$ H $-2.792928-4.258662 \quad 2.785053$

H $-2.342453 \quad-2.5417912 .676437$ H $-1.586560-3.590473 \quad 3.909226$ н $-2.516381-3.411994-3.247794$ H $-3.591715-5.086943-1.705483$ H $-2.805286-5.293156 \quad 0.622017$ C $3.2698731 .702550-1.207197$ C $3.043302 \quad 1.815204 \quad 1.243296$ C $4.001757 \quad 2.894348-1.157456$ C $3.7860112 .997491 \quad 1.235451$ C $4.2618993 .539728 \quad 0.046532$ C $3.019881 \quad 1.031873-2.548633$ C $2.1618451 .906211-3.475615$ $\begin{array}{llll}\text { C } & 2.161845 & 1.906211 & -3.475615 \\ \text { C } & 4.342146 & 0.667439 & -3.244123\end{array}$ $\begin{array}{llll}C & .342146 & 0.667439 & -3.244123 \\ \mathrm{H} & 2.457274 & 0.101628 & -2.387870\end{array}$ $\begin{array}{llll}\text { H } & 2.457274 & 0.101628 & -2.387870 \\ \text { H } & 1.175281 & 2.107862 & -3.036701\end{array}$ H $1.1752812 .107862-3.036701$ $\begin{array}{llll}\text { H } 2.656637 & 2.867590 & -3.688784\end{array}$ H $2.000618 \quad 1.381753-4.429600$ H $5.005392 \quad 0.084698 \quad-2.587022$ H $4.891754 \quad 1.570866-3.554914$ H $4.1338520 .070812-4.145376$ $\begin{array}{llll}\text { C } 2.556482 & 1.216966 & 2.553567\end{array}$ C $3.569373 \quad 0.206590 \quad 3.118186$ C $2.236822 \quad 2.267025 \quad 3.623263$ H $4.534897 \quad 0.701178 \quad 3.314002$ H $3.750674-0.6254932 .424656$ H $3.199729-0.214787 \quad 4.066638$ $\begin{array}{lllll}H & 3.199729 & -0.214787 & 4.066638\end{array}$ $\begin{array}{llll}\text { H } 3.152338 & 2.740402 & 4.012118\end{array}$ $\begin{array}{lllll}\mathrm{H} & 1.578682 & 3.059602 & 3.238370\end{array}$ H 1.7343641 .7858074 .476405 $\begin{array}{lllll}\text { H } & 4.374673 & 3.324027 & -2.089429\end{array}$ $\begin{array}{lllll}\text { H } & 4.835337 & 4.469925 & 0.057784\end{array}$ $\begin{array}{lllll}\text { H } 3.991118 & 3.509216 & 2.177528\end{array}$ $\begin{array}{llll}\text { C }-0.238994 & 1.939873 & -0.393120\end{array}$ H $-1.0494871 .929521-1.138936$ $\begin{array}{lllll}\text { H } & 0.714287 & 2.162357 & -0.894344\end{array}$ C $-0.497644 \quad 2.932034 \quad 0.717376$ $\begin{array}{llll}\text { C } & -0.927320 & 0.029731 & 1.722454\end{array}$ C $-1.875889-0.067913 \quad 0.693893$ H $-0.765244 \quad 0.973332 \quad 2.244803$ H $-0.609636 \quad-0.863837 \quad 2.266422$ $\begin{array}{lllll}\mathrm{H} & -2.458376 & 0.804967 & 0.391554\end{array}$ H $-2.305996-1.033473 \quad 0.410044$ $\begin{array}{lllll}H & -2.305996 & -1.033473 & 0.410044\end{array}$

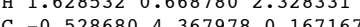
$\begin{array}{llll}\text { C } & -0.528680 & 4.367978 & 0.167167\end{array}$ H 0.2955802 .8714931 .479607 $\begin{array}{lllll}H & -1.457114 & 2.730987 & 1.223479\end{array}$ H -0.7301675 .0906670 .973925$ $\begin{array}{cccc}\mathrm{H} & 0.436780 & 4.626784 & -0.294561 \\ \mathrm{H} & -1.313068 & 4.478518 & -0.597959\end{array}$ 82

$17 \mathrm{R}=\mathrm{n}-\mathrm{Pr} \mathrm{AC} / \mathrm{pi}$

$\mathrm{Ni}-0.003682 \quad 0.003622 \quad-0.049322$ N $2.004721-0.0009850 .002406$ C $2.855194 \quad 1.159563 \quad 0.019726$ $\begin{array}{llll}\text { C } 2.540400 & -1.188839 & 0.106111\end{array}$ C $3.991955-1.468993 \quad 0.316725$ H $4.591941-0.552280 \quad 0.273376$ H $4.366816-2.177016-0.437707$ H $4.138281-1.943599-1.302423$ C $1.600414-2.311557-0.047440$ $\begin{array}{llll}C & 1.600414 & -2.311557 & -0.047440\end{array}$ C $2.118642-3.679063-0.333650$ H $2.767819-3.641448-1.223244$ H $2.717876-4.0586320 .508990$ H $1.299050-4.376316-0.541614$ $\mathrm{N} 0.344676-1.979197-0.021078$ C $-0.675977-2.905000-0.391964$ $\begin{array}{llll}\text { C } & -1.217042 & -2.721259 & -1.677646\end{array}$ $\begin{array}{llll}C & -1.186190 & -3.890346 & 0.469557\end{array}$ $\begin{array}{llll}\text { C }-2.259744 & -3.522071 & -2.125029\end{array}$ $\begin{array}{llll}\text { C }-2.235917 & -4.684118 & -0.013513\end{array}$ $\begin{array}{llll}\text { C }-2.768217 & -4.512012 & -1.285487\end{array}$ S $-0.533815-1.434748-2.761670$ o $0.885490-1.771106-2.941269$ $0-1.388273-1.402514-3.944657$ $0-0.732262-0.159706-1.927162$ $\begin{array}{lll}-0.732262 & -0.159706 & -1.927162\end{array}$ C $-0.116816-5.5248931 .880853$ $\begin{array}{llll}C-0.116816 & -5.524893 & 2.077932\end{array}$ C $-1.758381-3.8121662 .923958$ $\begin{array}{llll}\text { H } 0.149245 & -3.392244 & 2.061768\end{array}$ $0.668378-5.7675501 .346998$ H $0.308352-5.624054 \quad 3.088568$ H $-0.914070-6.277652 \quad 1.974667$ H $-2.167675-2.798303 \quad 2.80970$ H $-1.337825-3.8910103 .937497$ H $-2.590221-4.5291132 .835943$ H $-2.655641-3.352663-3.126431$ H $-3.586382-5.147745-1.624439$ $\begin{array}{llll}\text { H } & -2.650011 & -5.456650 & 0.635218\end{array}$ $\begin{array}{llll}\text { C } 3.181627 & 1.742306 & -1.22375\end{array}$ $\begin{array}{llll}\text { C } 3.294573 & 1.713685 & 1.235767\end{array}$ C $3.9683462 .898315-1.220222$ 
$\begin{array}{llll}\text { H } & 1.586681 & 2.842438 & -3.189109\end{array}$ H $1.865280 \quad 1.605144 \quad-4.442$ $\begin{array}{llll}\text { H } 3.180811 & 2.704485 & -3.976285\end{array}$ H $4.164640-0.566186-2.367725$ H $3.512479-0.304591-4.000444$ H $4.760870 \quad 0.801038 \quad-3.340316$ C $2.951073 \quad 1.122142 \quad 2.589672$ C $2.0656962 .077444 \quad 3.403466$ $\begin{array}{lllll}\text { C } 4.203977 & 0.758268 & 3.401147\end{array}$ H 2.3813120 .1 $\begin{array}{llll}\mathrm{H} & 2.381312 & 0.196805 & 2.433317\end{array}$ $\begin{array}{llll}1.184256 & 2.397200 & 2.829338\end{array}$ H $2.622632 \quad 2.986045 \quad 3.683670$ H 1.7238301 .5845164 .326151 H 4.8724200 .0863832 .841628 $\begin{array}{lllll}\text { H } & 4.781157 & 1.658213 & 3.669112\end{array}$ H $3.909153 \quad 0.254618 \quad 4.335203$ H $4.234332 \quad 3.361381 \quad-2.172005$ H $5.021867 \quad 4.376673-0.051907$ H $4.422794 \quad 3.331652 \quad 2.115626$ C $-0.088293 \quad 1.955524 \quad-0.361934$ H $0.550990 \quad 2.409686 \quad 0.412323$ H $0.428696 \quad 1.961791 \quad-1.331697$ C $-1.4403172 .618406-0.471131$ $\begin{array}{llll}\text { C } & -1.744794 & -0.139581 & 0.902070\end{array}$ $\begin{array}{llll}\text { C }-0.743435 & 0.288637 & 1.808934\end{array}$ H $-2.067844-1.183555 \quad 0.883747$

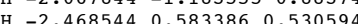
$\begin{array}{lllll}H & -2.468544 & 0.583386 & 0.530594\end{array}$ C -0.7065741 .3413672 .101826$ $\begin{array}{llll}C & -0.112998-0.581748 & 2.743168\end{array}$ $\mathrm{N} 0.438507-1.261577 \quad 3.519266$ C $-1.293140-4.068052-0.96811$ $\mathrm{H}-1.9583452 .641250 \quad 0.502517$ H $-2.0722342 .060714-1.180862$ H $-2.279725 \quad 4.557072-1.027172$ $\mathrm{H}-0.654160 \quad 4.657943-0.290697$ $\mathrm{H}-\mathrm{O}$

$7 \mathrm{R}=\mathrm{n}-\mathrm{Pr} \mathrm{AC} / \mathrm{N}-\mathrm{t}$

$\mathrm{Ni}-0.010472 \quad 0.005882 \quad-0.019174$ $\mathrm{N} 1.8969010 .004988-0.006363$ C $2.732154 \quad 1.170457 \quad 0.001339$ $\begin{array}{llll}\text { C } 2.472064 & -1.181270 & -0.128987\end{array}$ C $3.943382-1.41$ $\begin{array}{lll}C & 3.943382-1.416338 & -0.227182\end{array}$ $\begin{array}{lllll}\text { H } & 4.517330 & -0.522705 & 0.048404\end{array}$ H $4.214995-1.691210-1.261220$ $4.248620-2.2545900 .418398$ C $1.541782-2.290314-0.222490$ $\begin{array}{llll}\text { C } 2.006867-3.661545 & -0.575873\end{array}$ H $1.155529-4.326234-0.76745$ H $2.618337-3.620540-1.49023$ H $2.626892-4.0890450 .229143$ N $0.294205-1.943442-0.036091$ $\begin{array}{llll}\text { C }-0.781406 & -2.822240 & -0.328455\end{array}$ $\begin{array}{llll}\text { C }-1.367041 & -2.676282 & -1.598088\end{array}$ $\begin{array}{llll}\text { C }-1.302177 & -3.723536 & 0.611527\end{array}$ $\begin{array}{llll}\text { C }-2.471979 & -3.442798 & -1.948101\end{array}$ C $-2.413658-4.486340-0.228886$ C $-2.992876-4.354976-1.029747$ S $-0.663663-1.470208-2.771203$ $\begin{array}{llll}\mathrm{S} & -0.663663 & -1.470208 & -2.771203 \\ 0 & -1.553798 & -1.485807 & -3.932766\end{array}$ $\begin{array}{llll}0 & -1.553798 & -1.485807 & -3.932766\end{array}$ $\begin{array}{llll}0 & -0.769455 & -0.161269 & -2.001812\end{array}$ $\begin{array}{lllll}0 & 0.732637 & -1.896441 & -2.980313\end{array}$ C $-0.691864-3.8817521 .992711$ $\begin{array}{llll}C & -0.117344 & -5.291276 & 2.19984\end{array}$ C $-1.698608-3.5439063 .101154$ H $0.135942-3.1598972 .070448$ H $0.616070-5.549012 \quad 1.421969$ H $0.377505 \quad-5.3613693 .181264$ н $-0.916054-6.0494112 .17058$ н $-2.088849-2.522270 \quad 2.985871$ H $-1.217688-3.621222 \quad 4.088980$ H $-2.551437-4.240993 \quad 3.088041$ H $-2.904448-3.309128-2.940187$ H $-3.853248-4.968450-1.299488$ H $-2.832612-5.204034 \quad 0.936070$ C $3.2403021 .667397-1.215199$ $\begin{array}{llll}\text { C } & 3.240302 & 1.667397 & -1.215199 \\ \text { C } 2.963667 & 1.822905 & 1.227525\end{array}$ $\begin{array}{llll}\text { C } & 4.015476 & 2.830712 & -1.165117\end{array}$ $\begin{array}{lll}\text { C } 3.756585 & 2.972428 & 1.224597\end{array}$ $\begin{array}{llll}\text { C } 4.284638 & 3.477818 & 0.041080\end{array}$ $\begin{array}{llll}\text { C } & 4.284638 & 3.477818 & 0.041080 \\ \text { C } 2.943422 & 1.018778 & -2.557227\end{array}$ $\begin{array}{llll}\text { C } & 2.943422 & 1.018778 & -2.557227 \\ \text { C } 1.964520 & 1.874310 & -3.377325\end{array}$ $\begin{array}{llll}\text { C } & 1.964520 & 1.874310 & -3.377325 \\ \text { C } & 4.224866 & 0.752276 & -3.361326\end{array}$ $\begin{array}{llll}\text { C } & 4.224866 & 0.752276 & -3.361326 \\ \text { H } & 2.443489 & 0.054343 & -2.388894\end{array}$ $\begin{array}{llll}\text { H } & 2.443489 & 0.054343 & -2.388894 \\ \text { H } & 1.001065 & 1.979575 & -2.859944\end{array}$ H $1.001065 \quad 1.979575 \quad-2.859944$ H $2.3797752 .877768-3.565927$ H $1.768475 \quad 1.388140-4.345102$ H $4.971530 \quad 0.199186 \quad-2.770915$ H $4.6925891 .691666 \quad-3.697184$ H $3.982173 \quad 0.161704 \quad-4.257854$ $\begin{array}{llll}\text { C } 2.394610 & 1.277455 & 2.525456\end{array}$ C $1.9668542 .378737 \quad 3.501170$ $\begin{array}{lllll}C & 3.385450 & 0.317554 & 3.203477\end{array}$ C 3.3854500 .3175543 .203477 1.453530 .69685342 .262881 13.6434280 .5268742 .547690 $\begin{array}{llll}\text { H } & 2.952137 & -0.088609 & 4.131295\end{array}$
H $1.3181693 .119646 \quad 3.010796$ H $2.8344962 .908526 \quad 3.924516$ н $1.4140901 .935682 \quad 4.343681$ н $4.4178303 .240351-2.094053$ H $4.898476 \quad 4.381940 \quad 0.053608$ H $3.956408 \quad 3.489527 \quad 2.164918$ C $-0.169701 \quad 1.979086-0.016126$ H $0.724170 \quad 2.306585 \quad-0.566592$ C $-1.4286102 .522562-0.659757$ H $-0.0761482 .270906 \quad 1.043611$ $\begin{array}{llll}\mathrm{H} & -0.076148 & 2.270906 & 1.043611\end{array}$ $\begin{array}{llll}\mathrm{N} & -1.609891 & -0.071877 & 0.850400\end{array}$ $\begin{array}{llll}C & -2.682135 & -0.201983 & 1.291876\end{array}$ C $-3.981312-0.3150791 .826456$ C $-4.802313-1.3379061 .529816$ H $-4.294930 \quad 0.4896722 .497549$ $\mathrm{H}-5.801238-1.3853601 .96706$ $\begin{array}{lllll}\mathrm{H} & -4.491782 & -2.135202 & 0.849503\end{array}$ C $-1.351125 \quad 4.050180-0.81265$ $\begin{array}{lllll}\text { H }-2.318367 & 2.267427 & -0.063467\end{array}$ H $-1.5700702 .062195-1.649788$ H $-2.266614 \quad 4.439058-1.286516$ H $-1.233774 \quad 4.543157 \quad 0.165633$ $\mathrm{H}$

17

Ni $0.009425 \quad 0.004456-0.082504$ $\mathrm{N} 1.9703810 .006805-0.028001$ $1.97038130 .006805-0.028001$ C $2.7775131 .185363-0.028353$ C $3.984054-1.4614740 .062145$ H $4.562196-0.5368900 .184078$ H $4.290583-1.935903-0.885927$ H $4.244261-2.1615520 .871396$ $\begin{array}{llll}\text { C } 1.554290 & -2.266176 & -0.016535\end{array}$ $\begin{array}{llll}\text { C } 2.006792 & -3.663991 & -0.274862\end{array}$ н $2.575233-3.695279-1.217753$ H $2.667884-4.0144450 .534636$ H $1.160132-4.353600-0.370882$ $\begin{array}{llll}\mathrm{N} & 0.293592 & -1.884008 & 0.118303\end{array}$ C $-0.726527-2.825683-0.238231$ C $-1.142554-2.814597-1.580512$ C $-1.324968-3.679490 \quad 0.698963$ C $-2.152996-3.670034-2.003605$ $\begin{array}{llll}C & -2.152996 & -3.670034 & -2.003605\end{array}$ $\begin{array}{llll}C & -2.327321 & -4.543399 & 0.238819\end{array}$ $\begin{array}{llll}C & -2.737924 & -4.545952 & -1.089925\end{array}$ S $-0.381586-1.649080-2.757409$ $\begin{array}{llll}0 & 1.049863-2.009503 & -2.795671\end{array}$ $0-1.139790-1.789560-4.000871$ $0-0.625514-0.301397-2.095529$ $\begin{array}{llll}C & -0.915927 & -3.683974 & 2.161198\end{array}$ $\begin{array}{llll}\text { C }-0.183490 & -4.980095 & 2.54218\end{array}$ C $-2.119115 \quad-3.466175 \quad 3.089484$ H $-0.220074 \quad-2.842402 \quad 2.306828$ H $0.707660-5.144398 \quad 1.919972$ H $0.132206 \quad-4.943997 \quad 3.596771$ H $-0.846107 \quad-5.851416 \quad 2.416793$ H $-2.676187-2.558967 \quad 2.818209$ H $-1.780881-3.369360 \quad 4.133021$ $\mathrm{H}-2.816104-4.317800 \quad 3.043769$ $\begin{array}{lll}\mathrm{H}-2.816104 & -4.317800 & 3.043769\end{array}$ $\begin{array}{lllll}\mathrm{H} & -2.465087 & -3.630491 & -3.047580\end{array}$ $\begin{array}{llll}\mathrm{H} & -3.525189 & -5.226855 & -1.414585\end{array}$ $\begin{array}{llllll}\mathrm{H} & -2.804300 & -5.225194 & 0.944141\end{array}$ $\begin{array}{llll}\text { C } 3.229388 & 1.696167 & -1.26111 \\ \text { C } 3.033346 & 1.845821 & 1.187961\end{array}$ $\begin{array}{llll}\text { C } 3.03334611 & 1.845821 & 1.187961 \\ \text { C } 3.975611 & 2.877326 & -1.243961\end{array}$ $\begin{array}{llll}\text { C } 3.975611 & 2.877326 & -1.243961\end{array}$ C 3.7931583 .0203331 .154900 $\begin{array}{llll}\text { C } 4.269263 & 3.538375 & -0.045651\end{array}$ $\begin{array}{llll}\text { C } 2.897935 & 1.019493 & -2.578644\end{array}$ C $1.8323241 .818163-3.341165$ $\begin{array}{llll}\text { C } 4.138149 & 0.807697 & -3.456482\end{array}$ $\begin{array}{lllll}\text { H } 2.463261 & 0.031066 & -2.371708\end{array}$ H $0.909039 \quad 1.911362 \quad-2.752289$ H $2.2025692 .826908 \quad-3.589627$ H $1.576089 \quad 1.299264-4.277286$ $4.9366810 .279287-2.910702$ $4.9500810 .279287-2$. $\begin{array}{llll}H & 4.550041 & 1.279264 & -2.910702\end{array}$ $\begin{array}{lllll}\text { H } 3.864794 & 0.205563 & -4.337474\end{array}$ $\begin{array}{lll}C & .5291241 .3006912 .513158\end{array}$

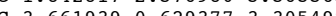
C 3.6619390 .6293773 .305498 H $2.558383 \quad 3.1404163 .706751$ н 1.0459792 .8820732 .797682 H 1.3942771 .9191394 .258508 H 4.4480251 .3604053 .555241 H $4.130848 \quad-0.183152 \quad 2.731266$ H $3.274664 \quad 0.208070 \quad 4.246679$ H $4.3419713 .287939-2.187399$ H $4.869450 \quad 4.452147 \quad-0.054323$ H $4.016802 \quad 3.538406 \quad 2.090542$ C $-1.892210 \quad-0.062998 \quad 0.475053$ $\begin{array}{llll}C & -2.086176 & 0.248815 & 1.943273\end{array}$ H $-2.3905450 .663202-0.185366$ H $-2.3905450 .663202-0.185366$ H $-2.232006-1.0745500 .209033$ $\mathrm{C}-0.1542040 .1966250 .052352$ $\begin{array}{lllll}C-0.246299 & 2.959541 & 0.028948\end{array}$ C $0.639812 \quad 5.212229-0.068237$

H $-1.429469 \quad 4.720822 \quad 0.042167$ H $1.670290 \quad 4.850824-0.114227$ H $0.463003 \quad 6.289106-0.086102$ H $1.773045 \quad 0.533759 \quad 2.285639$ C $-3.574909 \quad 0.243518 \quad 2.328700$ H $-1.659240 \quad 1.235035 \quad 2.190176$ н $-1.547676-0.487303 \quad 2.563822$ H $-3.705104 \quad 0.444505 \quad 3.403642$ H $-4.125433 \quad 1.012901 \quad 1.765363$ H $-4.040798 \quad-0.727274 \quad 2.100458$

$19 \mathrm{R}=\mathrm{Me}$

Ni $-0.154075 \quad-0.020468 \quad-0.011358$ N $1.690842 \quad 0.070954 \quad 0.018670$ $\begin{array}{llll}\text { C } 2.439590 & 1.305998 & 0.027826\end{array}$ $\begin{array}{llll}\text { C } 2.296730 & -1.100697 & -0.006136\end{array}$ $\begin{array}{llll}\text { C } 3.797928 & -1.276271 & 0.219448\end{array}$ F $4.266370-0.4011431 .137270$ F $4.530824-1.128445-0.907913$ $\begin{array}{llll}\text { F } 4.033016 & -2.523983 & 0.707225\end{array}$ $\begin{array}{llll}\text { C } 1.401076 & -2.255028 & -0.048905\end{array}$ $\begin{array}{llll}\text { C } 1.819768 & -3.577781 & -0.705742\end{array}$ F $2.800589-3.299729-1.612872$ F $2.300689-4.5261420 .134308$ F $0.785150-4.112186-1.380019$ F $0.785150-4.112186-1.380019$ $\begin{array}{llll}\mathrm{N} & 0.163191 & -1.951843 & 0.252099\end{array}$ $\begin{array}{llll}C & -0.845160 & -2.910695 & 0.504564\end{array}$ $\begin{array}{llll}C & -2.077615 & -2.726700 & -0.158688\end{array}$ C $-0.651857-3.9188871 .487561$ C $-3.078074-3.6724630 .057868$ $\begin{array}{llll}C & -1.698139 & -4.824891 & 1.664326\end{array}$ $\begin{array}{llll}C & -2.882525 & -4.735311 & 0.935637\end{array}$ $\begin{array}{llll}\text { B }-2.352068 & -1.437849 & -1.072997\end{array}$ F $-1.450775-1.244407-2.123555$ F $-3.669371-1.268309-1.432824$ F $-2.029793-0.236081-0.079970$ $\begin{array}{llll}\text { C } 0.502916 & -3.905654 & 2.478821\end{array}$ $\begin{array}{llll}\text { C } 0.881361 & -5.296896 & 2.996651\end{array}$ C $0.154299-2.964406 \quad 3.645475$ H $1.399904-3.497312 \quad 1.997435$ н $1.041486-6.0048152 .170814$ H $1.809692-5.2329563 .583320$ H $1.809692-5.2329563 .583320$ $\begin{array}{llll}\text { H } & 0.104936 & -5.704426 & 3.661292\end{array}$ $\mathrm{H}-0.053778-1.945680 \quad 3.284571$ H $-0.742301-3.3229074 .175719$ H $0.990565-2.920316 \quad 4.360912$ H $-4.028397-3.552050-0.463206$ $\begin{array}{llll}\text { H }-3.667865 & -5.476470 & 1.088946\end{array}$ H $-1.593754 \quad-5.613716 \quad 2.407998$ C $3.008221 \quad 1.765263-1.176344$ $\begin{array}{llll}\text { C } 2.470283 & 2.058013 & 1.215756\end{array}$ C $3.613132 \quad 3.027642-1.152048$ C 3.0993753 .3020661 .184415 C $3.662916 \quad 3.793031 \quad 0.011054$ $\begin{array}{llll}\text { C } 2.931992 & 1.004975 & -2.489321\end{array}$ $\begin{array}{llll}\text { C } 4.283639 & 1.045158 & -3.269792\end{array}$ $\begin{array}{llll}\text { C } 1.795477 & 1.532649 & -3.376738\end{array}$ H $2.719507-0.051968-2.279880$ H $4.4641892 .043686-3.699011$ H $4.241504 \quad 0.327321 \quad-4.104424$ $\begin{array}{llll}\text { H } & 5.132067 & 0.782997\end{array}$ н $0.817186 \quad 1.408213-2.895127$ H $1.778727 \quad 0.980727 \quad-4.329047$ H $1.939535 \quad 2.602231 \quad-3.597150$ C $1.859465 \quad 1.556185 \quad 2.513102$ c $0.659863 \quad 2.4159572 .937770$ C $2.8988341 .491802 \quad 3.642909$ H $1.493293 \quad 0.5317892 .342353$ H $-0.113455 \quad 2.441231 \quad 2.158678$ H $0.973988 \quad 3.452295 \quad 3.139108$ H $0.212326 \quad 2.011618 \quad 3.858638$ H $3.776604 \quad 0.897452 \quad 3.353150$ H 3.2452442 .5000673 .917366 H $2.448077 \quad 1.040184 \quad 4.539619$ H $4.0583393 .418739-2.069000$ H $4.0583393 .418739-2.069000$ H 3.1487693 .8979642 .007389 C $-0.5159651 .803276-0.500019$ $\begin{array}{llll}\text { C } & -0.515965 & 1.803276 & -0.500019 \\ \text { H } & -1.280907 & 2.143009 & 0.215464\end{array}$ $\begin{array}{llll}\text { H } & -1.280907 & 2.143009 & 0.215464 \\ \mathrm{H} & -0.965684 & 1.704273 & -1.500711\end{array}$ H $0.372309 \quad 2.445442-0.506072$ 75

$19 \mathrm{R}=\mathrm{Me} \mathrm{ET} / \mathrm{pi}$

Ni $0.112352-0.086950-0.109994$ N $2.089686-0.081677-0.106316$ C $2.838597 \quad 1.151379-0.032068$ $\begin{array}{llll}\text { C } 2.654161 & -1.259989 & -0.123850\end{array}$ $\begin{array}{llll}\text { C } 4.161937 & -1.501497 & 0.046134\end{array}$ F $4.905516-0.386129-0.046522$ F $4.630776-2.364619-0.882353$ 
$\begin{array}{llll}\text { N } 0.458321 & -2.054100 & -0.188646\end{array}$ C $-0.652903-2.872700-0.615643$ C $-1.027525-2.641933-1.952311$ $\begin{array}{llll}\text { C }-1.376414 & -3.687814 & 0.267687\end{array}$ C $-2.146273-3.334466-2.421340$ $\begin{array}{llll}\text { C }-2.490067 & -4.349079 & -0.264160\end{array}$ C $-2.869268-4.189579-1.592589$ B $-0.266713-1.548521-2.871814$ F $1.119646-1.774456-2.988548$ F $0.872528-1.360358-2.98548$ F $-0.872528-1.360358-4.105170$ $\begin{array}{llll}\text { F }-0.389500 & -0.219294 & -2.126906\end{array}$ $\begin{array}{llll}\text { C }-1.010120 & -3.888616 & 1.729072\end{array}$ C $-0.700350-5.360182 \quad 2.047633$ C $-2.122185-3.3850992 .664698$ H $-0.097196-3.3051001 .934546$ H $0.093832-5.759008 \quad 1.402872$ $\begin{array}{llll}\mathrm{H} & -1.597066 & -5.983021 & 1.905917\end{array}$ H $-0.386231-5.461627 \quad 3.097945$ H $-3.042715-3.9719062 .519058$ H $-2.370070-2.3295482 .487282$ H $-1.816369-3.499355 \quad 3.716497$ H $-2.447338-3.177092-3.458263$ H $-3.737894-4.725568-1.976931$ $\begin{array}{lllll}\text { H } & -3.070800 & -5.009165 & 0.381345\end{array}$ C $3.081051 \quad 1.828262-1.242685$ C $3.184985 \quad 1.696307 \quad 1.215068$ $\begin{array}{llll}\text { C } & 3.184985 & 1.696307 & 1.215068 \\ \text { C } 3.723848 & 3.066085 & -1.168893\end{array}$ $\begin{array}{llll}\text { C } 3.818131 & 2.941280 & 1.227990\end{array}$ $\begin{array}{llll}\text { C } & 3.818131 & 2.941280 & 1.227990 \\ \text { C } & 4.092427 & 3.624232 & 0.050915\end{array}$ $\begin{array}{llll}\text { C } & 4.092427 & 3.624232 & 0.050915 \\ \text { C } 2.693294 & 1.245791 & -2.589325\end{array}$ $\begin{array}{llll}\text { C } & 2.693294 & 1.245791 & -2.589325 \\ \text { C } & 1.886015 & 2.233871 & -3.442213\end{array}$ $\begin{array}{llll}\text { C } & 1.886015 & 2.233871 & -3.442213 \\ \text { C } 3.934533 & 0.759571 & -3.354667\end{array}$ $\begin{array}{lllll}\text { C } & 3.934533 & 0.759571 & -3.354667 \\ H & 2.051106 & 0.370860 & -2.422933\end{array}$ $\begin{array}{llll}\mathrm{H} & 2.051106 & 0.370860 & -2.422933 \\ \mathrm{H} & 1.010716 & 2.613362 & -2.896669\end{array}$ $\begin{array}{lllll}\text { H } & 1.010716 & 2.613362 & -2.896669 \\ \text { H } & 2.498074 & 3.093753 & -3.757637\end{array}$ H $1.526351 \quad 1.723954-4.347923$ H $4.512377 \quad 0.027979-2.771519$ H $4.6017561 .603495 \quad-3.593701$ H $3.624980 \quad 0.281250 \quad-4.295697$ $\begin{array}{llll}\text { C } 2.903649 & 0.999509 & 2.534607\end{array}$ C $4.205954 \quad 0.646593 \quad 3.277733$ C $1.995408 \quad 1.851583 \quad 3.433925$ H $4.741256 \quad 1.562235 \quad 3.576097$ 4.7425831 .5622353 .576 $\begin{array}{lllll}\mathrm{H} & 4.882583 & 0.049191 & 2.652047\end{array}$ H 3.9776910 .0751594 .191197 H 2.5174972 .7614553 .768865 H 1.0863602 .1676782 .903187 $\begin{array}{llll}\mathrm{H} & 1.704697 & 1.283624 & 4.331124 \\ \mathrm{H} & 3.933609 & 3.606374 & -2.094170\end{array}$ $\begin{array}{lllll}\text { H } & 3.933609 & 3.606374 & -2.094170 \\ \text { H } & 4.590335 & 4.596207 & 0.081217\end{array}$ $\begin{array}{lllll}\mathrm{H} & 4.590335 & 4.596207 & 0.081217 \\ \mathrm{H} & 4.101738 & 3.382303 & 2.185963\end{array}$ $\begin{array}{lllll}\text { H } & 4.101738 & 3.382303 & 2.185963 \\ \text { C } & -0.167525 & 1.843131 & -0.235532\end{array}$ H $-0.7023601 .914463-1.193965$ H $0.822170 \quad 2.313634-0.272450$ H $-0.765993 \quad 2.261788 \quad 0.583557$ $\begin{array}{llll}\text { C }-0.756757 & -0.091605 & 1.706562\end{array}$ C $-1.733979 \quad-0.178913 \quad 0.706809$ $\begin{array}{llll}\text { H } & -0.572980 & 0.848918 & 2.228214\end{array}$ $\begin{array}{llll}\mathrm{H} & -0.408243 & -0.988676 & 2.224687\end{array}$ H -2.3228740 .6965290 .426572$ $\begin{array}{llll}\mathrm{H} & -2.322874 & 0.696529 & 0.426572 \\ \mathrm{H} & -2.155088 & -1.142725 & 0.407249\end{array}$ $\begin{array}{llll}\mathrm{H} & -2.155088 & -1.142725 & 0.407249 \\ \mathrm{H} & 2.372379 & 0.058895 & 2.322682\end{array}$ 76

$19 \mathrm{R}=\mathrm{Me} \mathrm{AC} / \mathrm{pi}$

Ni $0.104684-0.089504-0.015245$ N $2.093832-0.077450-0.023307$ $\begin{array}{llll}\text { C } 2.831286 & 1.166743 & -0.009815\end{array}$

$\begin{array}{llll}\text { C } 2.645903 & -1.255168 & 0.053410\end{array}$ $\begin{array}{llll}\text { C } 4.147806 & -1.491870 & 0.300037\end{array}$ $\begin{array}{llll}\text { F } 4.890553 & -0.376680 & 0.204560\end{array}$ $\begin{array}{llll}\text { F } 4.662184 & -2.380158 & -0.578744\end{array}$ F $4.304612-1.9894971 .556699$ C $1.696707-2.395775-0.074282$ C $2.201429-3.819476-0.391427$ F $2.814818-3.847241-1.590018$ F $3.088924-4.2023480 .569493$ $\begin{array}{llll}\text { F } & 3.088924 & -4.202348 & 0.569493 \\ \text { N } 0.21930 & -4.734353 & -0.410736\end{array}$ $\begin{array}{llll}\mathrm{F} & 1.221930 & -4.734353 & -0.410736 \\ \mathrm{~N} & 0.447689 & -2.073057 & 0.003168\end{array}$ $\begin{array}{llll}\mathrm{N} & 0.447689 & -2.073057 & 0.003168\end{array}$ $\begin{array}{llll}\text { C } & -0.654943 & -2.930475 & -0.384661 \\ \text { C }-1.070467 & -2.734494 & -1.715218\end{array}$ $\begin{array}{llll}\text { C }-1.070467 & -2.734494 & -1.715218 \\ \text { C }-1.320678 & -3.768591 & 0.523794\end{array}$ $\begin{array}{llll}\text { C }-1.320678 & -3.768591 & 0.523794 \\ \text { C }-2.174801 & -3.471567 & -2.148643\end{array}$ $\begin{array}{llll}\text { C } & -2.174801 & -3.471567 & -2.148643 \\ \text { C }-2.424449 & -4.472566 & 0.025860\end{array}$ $\begin{array}{llll}\text { C } & -2.424449 & -4.472566 & 0.025860 \\ \text { C }-2.846627 & -4.340014 & -1.291826\end{array}$ $\begin{array}{llll}\text { B }-0.354681 & -1.659490 & -2.681865\end{array}$ F $1.030759-1.854956-2.825253$ F $-0.995300-1.495539-3.898523$ F $-0.484067-0.303824-1.958607$ $\begin{array}{llll}\text { C }-0.908749 & -3.955084 & 1.973789\end{array}$ C $-0.548600-5.416265 \quad 2.286990$ C $-2.012172-3.4827872 .934709$ H $-0.015460-3.3424352 .166028$ H $-0.015460-3.3424352 .166028$ H $0.237971-5.7955561 .621110$ $\begin{array}{llll}\mathrm{H}-0.197124 & -5.497532 & 3.326917\end{array}$ H $-1.428976-6.0686672 .178757$ $\begin{array}{llll}\text { H } & -2.302395 & -2.437538 & 2.755890 \\ \mathrm{H} & -1.663863 & -3.559547 & 3.975426\end{array}$
H $-2.914686-4.104806 \quad 2.825261$ H $-2.503710-3.341902-3.180825$ H $-3.705577-4.910412-1.646969$ н $-2.960126-5.148251 \quad 0.693495$ C $3.034124 \quad 1.790479-1.257385$ C $3.205815 \quad 1.770800 \quad 1.202745$ C $3.650290 \quad 3.043894 \quad-1.258253$ C $3.8026383 .034334 \quad 1.139062$ C $4.0288393 .668848-0.074227$ C $2.6586751 .113869-2.562098$ $\begin{array}{llll}\text { C } 2.658675 & 1.113869 & -2.562098\end{array}$

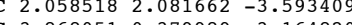
$\begin{array}{llll}\text { C } 3.868051 & 0.379989 & -3.164820\end{array}$ $1.8954230 .351374-2.352260$ H $1.2534872 .694712-3.161094$ H $1.6378951 .504482-4.430038$ H $2.8216382 .760407-4.006878$ H $4.280118-0.370885-2.476158$ H $3.565606-0.137891-4.086893$ H $4.6728691 .093834-3.404076$ C $2.9894121 .134165 \quad 2.563875$ C $2.053004 \quad 1.983034 \quad 3.434745$ C $4.312475 \quad 0.913546 \quad 3.314093$ H $2.517702 \quad 0.150252 \quad 2.428934$ H $1.120515 \quad 2.236368 \quad 2.910871$ н $2.536203 \quad 2.931806 \quad 3.716902$ H $1.799899 \quad 1.4404864 .357550$ $\begin{array}{lllll}\mathrm{H} & 1.799899 & 1.440486 & 4.357550\end{array}$ $\begin{array}{llll}H & 5.026608 & 0.324504 & 2.721635\end{array}$ $\begin{array}{lllll}\text { H } & 4.788997 & 1.876553 & 3.560582\end{array}$ 1.1184680 .378 $\begin{array}{lllll}\text { H } 3.829293 & 3.543694 & -2.211932\end{array}$ H $4.5012104 .653663-0.100650$ H $4.099642 \quad 3.525065 \quad 2.068363$ $\begin{array}{llll}C & -0.087361 & 1.834448 & -0.313310\end{array}$ H $0.627001 \quad 2.367315 \quad 0.326819$ н $0.1737921 .893870-1.377026$ H $-1.119887 \quad 2.164847-0.142919$ $\begin{array}{llll}\text { C }-1.680064 & -0.188452 & 0.872657\end{array}$ $\begin{array}{llll}\text { C }-0.705180 & 0.240946 & 1.802300\end{array}$ $\begin{array}{llll}\text { C }-0.099153 & -0.599569 & 2.781588\end{array}$ N $0.438010-1.235125 \quad 3.601761$ $\mathrm{H}-2.015110-1.227655 \quad 0.836956$ H $-2.3648810 .548294 \quad 0.451019$ $\begin{array}{llll}\mathrm{H} & -0.660271 & 1.301027 & 2.064942\end{array}$ 76

$19 \mathrm{R}=\mathrm{Me} \mathrm{AC} / \mathrm{N}$

Ni $0.007309-0.008552-0.052764$ N $1.8923510 .019640-0.082368$ C $2.623366 \quad 1.261243-0.035847$ C $2.479118-1.152918-0.250749$ C $3.994998-1.355950-0.252585$ $\begin{array}{lllll}\text { F } 4.667836 & -0.339961 & 0.321962\end{array}$ F $4.480829-1.501883-1.508646$ $\begin{array}{llll}\text { F } 4.299693 & -2.481042 & 0.452782\end{array}$ $\begin{array}{llll}\text { C } 1.543303 & -2.269278 & -0.412425\end{array}$ $\begin{array}{llll}\text { C } 1.977559 & -3.693492 & -0.780676\end{array}$ F $1.003474-4.385927-1.389173$ F $3.049001-3.687331-1.603212$ F $2.319765-4.3720790 .355229$ N $0.300191-1.932680-0.239367$ C $0.300191-1.932680-0.239367$ $\begin{array}{llll}C & -0.859152 & -2.724077-0.550447\end{array}$ $\begin{array}{llll}C & -1.456472 & -2.399208 & -1.782792\end{array}$ C $-1.405578-3.6287070 .369760$ C $-2.635224-3.074993-2.105090$ C $-2.598910-4.260303-0.005016$ $\begin{array}{llll}C & -3.206434 & -3.998624 & -1.229076\end{array}$ B $-0.810284-1.290007-2.779637$ F $-1.575857-1.101257-3.926581$ F $-0.812250 \quad 0.023476-2.036579$ F $0.542886-1.560368-3.079779$ C $-0.769909 \quad-3.938956 \quad 1.713094$ $\begin{array}{llll}\text { C }-0.519202 & -5.443635 & 1.887853\end{array}$ C $-1.618812 \quad-3.397136 \quad 2.873142$ H $0.205540-3.430609 \quad 1.756733$ н $0.055504-5.854075 \quad 1.045894$ H $0.039083-5.6303062 .818068$ H $-1.469220-5.9964211 .952139$ $\mathrm{H}-1.469220-5.996421 \quad 1.952139$ $\mathrm{H}-1.745142-2.3070632 .799697$ - $-1.139530-3.6256353 .838167$ H $-2.618637-3.8598642 .873085$ $\mathrm{H}-3.100982-2.858654-3.068232$ H $-4.123812-4.522878-1.502406$ H $-3.050976-4.9841810 .674584$ $\begin{array}{llll}\text { C } 3.047578 & 1.841661 & -1.246265\end{array}$ C 2.7303401 .9270831 .202095 C $3.5829263 .134345-1.180466$ C $3.294022 \quad 3.202333 \quad 1.206909$ C $3.701854 \quad 3.815362 \quad 0.023691$ $\begin{array}{llll}\text { C } 2.901054 & 1.172449 & -2.602121\end{array}$ $\begin{array}{llll}\text { C } 1.812395 & 1.855408 & -3.444584\end{array}$ C $4.2379961 .146362-3.359409$ H $2.5769330 .132005-2.462082$ H $0.830284 \quad 1.795885-2.956173$ H $2.0606652 .914211-3.623256$ $5.7275711 .347087-4.416485$ $4.05391272 .758058-3.673475$ $\begin{array}{llll}\text { H } & 4.539127 & 2.158058 & -3.673475 \\ \mathrm{H} & 4.135202 & 0.532091 & -4.266477\end{array}$

C $2.246485 \quad 1.281793 \quad 2.492444$ $\begin{array}{llll}\text { C } 1.719877 & 2.298730 & 3.510933\end{array}$ C $3.327764 \quad 0.407859 \quad 3.151855$ H $1.406152 \quad 0.619245 \quad 2.224234$ H $3.637022 \quad-0.429102 \quad 2.513694$ H $4.223968 \quad 1.008489 \quad 3.375254$ H $2.946121-0.0085854 .097183$ H 1.0032883 .0001313 .058962 H $2.5394362 .883442 \quad 3.956813$ H 1.2167613 .7688004 .333880 H $3.9034303 .618088 \quad-333880$ H $3.9034303 .618088-2.104895$ $\mathrm{H}$
$4.123233 \quad 4.8227880 .044247$ C -0.2425281 .9330910 .055693$

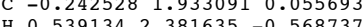
H $0.5391342 .381635-0.568737$ H $-1.2446162 .124800-0.34978$ H $-0.162153 \quad 2.241210 \quad 1.106034$ $\begin{array}{llll}\mathrm{N} & -1.630574 & -0.129124 & 0.764884\end{array}$ $\begin{array}{llll}\text { C }-2.733419 & -0.224863 & 1.122445\end{array}$ C $-4.070423-0.333103 \quad 1.556684$ C $-4.907179-1.240298 \quad 1.015902$ $\begin{array}{llll}\text { H }-4.390168 & 0.362631 & 2.335970\end{array}$ н $-5.942047-1.3053281 .357678$ 81 $19 \mathrm{R}=\mathrm{n}-\mathrm{Pr} \mathrm{Et} / \mathrm{pi}$

Ni $0.051438 \quad 0.062850 \quad 0.054915$ $\begin{array}{lllll}N & 2.030809 & 0.012521 & 0.046758\end{array}$ $\begin{array}{lllll}\mathrm{N} & 0.453461 & 2.019435 & 0.108683\end{array}$ C 1.7077642 .3286600 .207708 C $2.625397 \quad 1.1792260 .076633$ C $-0.121985-1.896774 \quad 0.243360$ $\begin{array}{llll}\text { C }-1.472876 & -2.566732 & 0.335140\end{array}$ $\begin{array}{llll}\text { C }-1.302065 & -4.052107 & 0.70000\end{array}$ H $0.503828-2.327873-0.552438$ H $0.406656-1.946217 \quad 1.206576$ H $-2.017513-2.510707-0.621923$ H $-2.089614-2.078891 \quad 1.106935$ H $-0.696466-4.577471-0.053775$ H $-0.802357-4.163861 \quad 1.673257$ н $-2.284044 \quad-4.545541 \quad 0.760271$ C $-1.792457 \quad 0.267790-0.794437$ C $-0.842828-0.059813-1.762473$ $\begin{array}{ccc}C-0.842828 & -0.059813-1.762473\end{array}$ $\begin{array}{llll}\mathrm{H} & -0.0778666 & -1.074664 & -2.158671\end{array}$ $\begin{array}{lll}-1 & -0.389399 & 0.717464-2.384487\end{array}$ H $-2.471397-0.483380-0.394623$ $\begin{array}{llll}\mathrm{H} & -2.093207 & 1.303974 & -0.624129\end{array}$ $\begin{array}{lllll}C & 2.756738 & -1.234397 & -0.017794\end{array}$ $\begin{array}{llll}C & -0.641410 & 2.888711 & 0.454395\end{array}$ $\begin{array}{lll}\text { C } 2.198031 & 3.767225 & 0.454849\end{array}$ $\begin{array}{llll}\text { C } 4.145946 & 1.376726 & 0.015179\end{array}$ $\begin{array}{lllll}\text { F } 4.806574 & 0.312693 & -0.477994\end{array}$ F $4.4366402 .432275-0.793291$ F $4.654674 \quad 1.625902 \quad 1.244835$ F $2.506097 \quad 4.337727-0.748937$ F $3.3041553 .795351 \quad 1.227275$ F $1.277555 \quad 4.539784 \quad 1.047238$ C $-1.1453312 .658862 \quad 1.748273$ C $-1.227766 \quad 3.761393 \quad-0.471756$ $\begin{array}{llll}C & -1.227766 & 3.761393 & -0.471756\end{array}$ $\begin{array}{llll}\text { C }-2.355082 & 4.469744 & -0.035032\end{array}$ $\begin{array}{llll}C & -2.355082 & 4.469744 & -0.035032\end{array}$ $\begin{array}{llll}C & -2.866126 & 4.304472 & 1.247072\end{array}$ $\begin{array}{llll}\text { B } & 0.906920 & 1.767758 \quad 2.932148\end{array}$ F $-1.163524 \quad 1.413843 \quad 3.928669$ F $-0.572925 \quad 0.2119392 .018723$ C $-0.712548 \quad 3.950911-1.887175$ C $-0.3103475 .418566-2.153968$ C $-1.737828 \quad 3.487859-2.934055$ H $0.193092 \quad 3.334520-2.007049$ н $0.4006215 .783761-1.399763$ H $-1.197684 \quad 6.071426-2.132086$ H $0.151366 \quad 5.508943-3.150261$ H $-2.653232 \quad 4.097601 \quad-2.878066$ $\mathrm{H}-2.029028 \quad 2.437700 \quad-2.789057$ $\mathrm{H}-1.322932 \quad 3.597082 \quad-3.948434$ H $-2.663633 \quad 3.2562863 .130206$ $\begin{array}{llll}\mathrm{H} & -2.663633 & 3.256286 & 3.130206 \\ \mathrm{H}-3.742298 & 4.874535 & 1.558448\end{array}$ $\begin{array}{llll}H & -3.742298 & 4.874535 & 1.558448 \\ \mathrm{H} & -2.840170 & 5.166924 & -0.719473\end{array}$ $\begin{array}{llll}C & 3.074849 & -1.882301 & 1.190744\end{array}$ C $2.997584-1.826408-1.273365$ C $3.663701-3.1519541 .102008$ c $3.593678-3.086998-1.297720$ $\begin{array}{llll}\text { C } 3.923059 & -3.753066 & -0.12321\end{array}$ $\begin{array}{llll}\text { C } 2.846454 & -1.260857 & 2.560121\end{array}$ C $2.000673-2.1522453 .483109$ $\begin{array}{llll}\text { C } 4.189362 & -0.951239 & 3.244325\end{array}$ H $2.300930 \quad-0.313238 \quad 2.438196$ H $1.026520-2.401934 \quad 3.041603$ H $2.523368-3.092838 \quad 3.718988$ H $1.811589-1.621748 \quad 4.428028$ $4.81589-1.6217480 .4302072 .608515$ H $4.725724-1.8834843 .483539$ $\begin{array}{llll}4.725724 & -1.883484 & 3.483539\end{array}$ 
H $4.487758-1.671664-3.653446$ H $4.576440-0.103480-2.822932$ H $3.628056-0.265204-4.325520$ $\begin{array}{llll}\text { H } & 2.276287 & -2.916741 & -3.820623\end{array}$ H $0.854476-2.368136-2.909770$ н $1.405353-1.460777-4.345785$ H $3.923255-3.6756432 .023986$ $\begin{array}{llll}\mathrm{H} & 4.383299 & -4.743137 & -0.162232 \\ \mathrm{H} & 3.801314 & -3.558165 & -2.260735\end{array}$ $\begin{array}{llll}\text { H } 2.106432 & -0.219588 & -2.364543\end{array}$ 82

$19 \mathrm{R}=\mathrm{n}-\mathrm{Pr} \mathrm{AC} / \mathrm{pi}$

Ni $0.077187-0.087383 \quad 0.016377$ N $2.077462-0.0737210 .007419$ $\begin{array}{llll}\mathrm{N} & 0.437024 & -2.074760 & -0.028977\end{array}$ $\begin{array}{llll}C & 1.687265 & -2.392231 & -0.088234\end{array}$ C $2.627988-1.2528030 .088578$ C $-0.072725 \quad 1.872444 \quad-0.256889$ $\begin{array}{llll}\text { C } & -1.427235 & 2.536134 & -0.279097\end{array}$ C $-1.279977 \quad 4.020808 \quad-0.659264$ H $0.606748 \quad 2.319145 \quad 0.484551$ H $0.392339 \quad 1.872916-1.254367$ H $-1.920481 \quad 2.488815 \quad 0.706322$ н $-2.0831602 .040208-1.012209$ H $-0.831038 \quad 4.128999-1.656986$ H $-0.639525 \quad 4.550517 \quad 0.061679$ $\begin{array}{llll}\mathrm{H} & -0.639525 & 4.550517 & 0.061679\end{array}$ $\begin{array}{llll}\mathrm{H} & -2.266012 & 4.509135 & -0.670638\end{array}$ $\begin{array}{llll}\text { C } & -1.677806 & -0.274605 & 0.961158\end{array}$ $\begin{array}{llll}C & -0.694336 & 0.180695 & 1.865598\end{array}$ C $-0.028514-0.658503 \quad 2.806883$ $\mathrm{N} 0.557242-1.3021113 .586843$ H $-1.966619-1.327166 \quad 0.922725$ $\begin{array}{lllll}\text { H } & -2.405975 & 0.432307 & 0.567906\end{array}$ H $-0.682539 \quad 1.236145 \quad 2.149011$ $\begin{array}{llll}\text { C } 2.825334 & 1.164209 & -0.013064\end{array}$ C $-0.666152-2.930977-0.403178$ $\begin{array}{llll}\text { C } 2.208828 & -3.790143 & -0.474160\end{array}$ $\begin{array}{llll}\text { C } 4.110591 & -1.496928 & 0.426287\end{array}$ F $4.170977-2.017194 \quad 1.683380$ F $4.858262-0.381078 \quad 0.405990$ F $4.692889-2.371955-0.422433$ F $2.895516-3.720729-1.634076$ $\begin{array}{llll}\text { F } 3.037974 & -4.252357 & 0.503578\end{array}$ $\begin{array}{lllll}\text { F } 1.234550 & -4.695741 & -0.632680\end{array}$ $\begin{array}{llll}\text { C }-1.162141 & -2.680695 & -1.696392\end{array}$ $\begin{array}{llll}C & -1.266179 & -3.821400 & 0.502452\end{array}$ $\begin{array}{llll}C & -2.278490 & -3.416136 & -2.09934\end{array}$

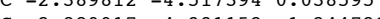
$\begin{array}{llll}\text { C }-2.889917 & -4.331152 & -1.244721\end{array}$ B $-0.494238-1.577325-2.665080$ F $-0.579354-0.236590-1.906989$ F $0.880888-1.779149-2.880216$ F $-1.193156-1.380020-3.844796$ $\begin{array}{llll}\text { C }-0.774832 & -4.053023 & 1.919725\end{array}$ $\begin{array}{llll}\text { C } & -0.351474 & -5.527337 & 2.153368\end{array}$ $\begin{array}{llll}\text { C }-1.836824 & -3.655560 & 2.956746\end{array}$ H $0.110367-3.42421122 .095255$ H $0.411365-5.8499161 .430611$ H $0.055413-5.6373013 .171433$ H $-1.219357 \quad-6.199919 \quad 2.058871$ $\begin{array}{llll}\text { H } & -1.219357 & -6.199919 & 2.058871 \\ \mathrm{H} & -2.166180 & -2.613488 & 2.835201\end{array}$ $\begin{array}{llll}\text { H } & -2.166180 & -2.613488 & 2.835201 \\ \text { H }-1.428919 & -3.760701 & 3.973016\end{array}$ $\begin{array}{llll}\text { H }-1.428919 & -3.760701 & 3.973016 \\ \text { H }-2.725431 & -4.300901 & 2.872245\end{array}$ $\begin{array}{llll}\text { H } & -2.725431 & -4.300901 & 2.872245 \\ \text { H } & -2.666207 & -3.249492 & -3.105513\end{array}$ $\begin{array}{llll}\text { H } & -2.666207 & -3.249492 & -3.105513 \\ \mathrm{H} & -3.762799 & -4.895708 & -1.574765\end{array}$ $\begin{array}{llll}\text { H } & -3.762799 & -4.895708 & -1.574765 \\ \text { H } & -2.879910 & -5.226926 & 0.706168\end{array}$ $\begin{array}{lllll}\text { H } & -2.879910 & -5.226926 & 0.706168 \\ \text { C } & 3.071675 & 1.717164 & -1.286398\end{array}$ $\begin{array}{llll}\text { C } 3.071675 & 1.717164 & -1.286398\end{array}$ $\begin{array}{llll}\text { C } 3.169122 & 1.832118 & 1.174043\end{array}$ $\begin{array}{llll}\text { C } 3.691332 & 2.967643 & -1.337734\end{array}$ $\begin{array}{llll}\text { C } 3.778111 & 3.085542 & 1.059459\end{array}$ C $4.038731 \quad 3.654781-0.179793$ $\begin{array}{llll}\text { C } 2.744530 & 0.965995 & -2.564082\end{array}$ $\begin{array}{llll}\text { C } 2.173257 & 1.863368 & -3.668412\end{array}$ $\begin{array}{lllll}\text { C } 3.983908 & 0.215323 & -3.080682\end{array}$ $\begin{array}{lllll}\text { H } & 1.979255 & 0.209082 & -2.342052\end{array}$ $\begin{array}{lllll}\mathrm{H} & 1.979255 & 0.209082 & -2.342052\end{array}$ $\begin{array}{llll}\mathrm{H} & 1.328373 & 2.467973 & -3.306920\end{array}$ $\begin{array}{lllll}\mathrm{H} & 1.810726 & 1.233290 & -4.493683\end{array}$ H $2.9352932 .545024-4.078029$ H $4.392492-0.475860-2.329970$ H $3.718660-0.370933-3.972934$ $\begin{array}{llll}\text { H } & 4.782410 & 0.926853 & -3.347045\end{array}$ $\begin{array}{llll}\text { C } 2.921755 & 1.259512 & 2.557834\end{array}$ $\begin{array}{llll}\text { C } 1.959161 & 2.139059 & 3.366508\end{array}$ C $4.225037 \quad 1.083913 \quad 3.352812$ H $2.463068 \quad 0.265846 \quad 2.454966$ H $1.035296 \quad 2.358303 \quad 2.812768$ H $2.427407 \quad 3.105165 \quad 3.613285$ H $1.6920081 .639062 \quad 4.309534$ H $4.956504 \quad 0.4687392 .809429$ H $4.691768 \quad 2.060412 \quad 3.563501$ H $4.0076980 .596040 \quad 4.316536$ H $3.900907 \quad 3.412602-2.312099$ $\begin{array}{llll}\mathrm{H} & 3.900907 & 3.412602 & -2.312099 \\ \mathrm{H} & 4.516287 & 4.635176 & -0.245865\end{array}$ $\begin{array}{llll}\mathrm{H} & 4.516287 & 4.635176 & -0.245865 \\ \mathrm{H} & 4.056711 & 3.622592 & 1.968710\end{array}$ 82 $19 \mathrm{R}=\mathrm{n}-\mathrm{Pr} \quad \mathrm{AC} / \mathrm{N}$

$\mathrm{Ni}-0.088706-0.1920510 .147801$
N $1.094321-0.103228-1.394265$ $\mathrm{N}-1.424161-0.224598-1.222684$ C $-0.964139-0.026850-2.439550$ $\begin{array}{llll}\text { c } 0.482458 & -0.126599 & -2.557900\end{array}$ $\begin{array}{llll}\text { C }-4.456676 & -2.614606 & -2.658489\end{array}$ $\begin{array}{llll}\text { C } 2.521626 & -0.140145 & -1.235341\end{array}$ $\begin{array}{lllll}\text { C } & -2.792715 & 0.094046 & -0.868689\end{array}$ $\begin{array}{lllll}\text { C }-1.852077 & 0.308910 & -3.645585\end{array}$ C $1.216361-0.215426-3.894946$ F $1.4675931 .018949-4.398799$ F $1.4675931 .018949-4.398799$ F $2.4043290 .850160-3.786305$ F $0.484790-0.908082-4.805094$ F $-2.158546-0.843994-4.314367$ $\begin{array}{llll}\mathrm{F}-1.192836 & 1.124761 & -4.502381\end{array}$ $\mathrm{F}-3.0039740 .911339-3.329718$ $\begin{array}{llll}C & -2.977900 & 1.389796 & -0.353569\end{array}$ $\begin{array}{llll}\text { C }-3.807036 & -0.870282 & -0.946136\end{array}$ $\begin{array}{llll}\text { C }-4.272631 & 1.726618 & 0.043930\end{array}$ C $-5.084301-0.468580-0.533329$ $\begin{array}{llll}\text { C } & -5.322655 & 0.813490 & -0.052616\end{array}$ B $-1.739764 \quad 2.422270 \quad-0.172241$ F $-2.123014 \quad 3.616017 \quad 0.429817$ F $-0.762443 \quad 1.753338 \quad 0.766903$ F $-1.0280502 .640461-1.374969$ C $-3.584066-2.289994-1.436593$ C $-3.845194-3.314334-0.320438$ $\begin{array}{llll}C & -3.845194 & -3.314334 & -0.320438 \\ \mathrm{H} & -2.528461 & -2.384003 & -1.738503\end{array}$ $\begin{array}{llll}\mathrm{H} & -2.528461 & -2.384003 & -1.738503\end{array}$ $\begin{array}{llll}\mathrm{H} & -4.310124 & -1.885388 & -3.466402 \\ \mathrm{H} & -4.214375 & -3.617717 & -3.042401\end{array}$ $\begin{array}{llll}\mathrm{H} & -4.214375 & -3.617717 & -3.042401\end{array}$ $\mathrm{H}-5.523524-2.609236-2.385952$ H $-3.230850-3.1100170 .567449$ H $-3.622804-4.332501-0.676761$ H $-4.901755-3.289372-0.010024$ H $-4.443882 \quad 2.730566 \quad 0.435973$ H $-6.329880 \quad 1.098127 \quad 0.255057$ H $-5.908291-1.180870-0.592049$ C $3.2024251 .090854-1.198776$ $\begin{array}{llll}\text { C } 3.167744 & -1.368919 & -1.000873\end{array}$ $\begin{array}{llll}\text { C } 4.582547 & 1.052967 & -0.958532\end{array}$ $\begin{array}{llll}\text { C } 4.543223 & -1.346184 & -0.764653\end{array}$ $\begin{array}{llll}\text { C } 5.252447 & -0.149462 & -0.749535\end{array}$ C $2.4946562 .421268-1.385711$ C $2.3810513 .171479-0.050256$ C $2.3810513 .171479-0.050256$ $\begin{array}{lllll}\text { C } 3.179293 & 3.294957 & -2.445484\end{array}$ $\begin{array}{llll}\mathrm{H} & 1.466783 & 2.233087 & -1.726042\end{array}$ $\begin{array}{lll}1.825193 & 2.582819 & 0.693205\end{array}$ $\begin{array}{lll}3.379834 & 3.407828 & 0.352861\end{array}$ $\begin{array}{llll}\text { H } & 1.832758 & 4.113322 & -0.200334\end{array}$ H $3.3096582 .749870-3.392242$ H $4.169134 \quad 3.641081-2.107136$ H $2.562301 \quad 4.185581-2.639631$ $\begin{array}{llll}\text { C } 2.433619 & -2.701140 & -1.018590\end{array}$ $\begin{array}{lllll}\text { C } 2.558207 & -3.435982 & 0.324583\end{array}$ $\begin{array}{llll}\text { C } 2.920378 & -3.610307 & -2.158505\end{array}$ H $1.364212-2.497832-1.183066$ H $2.810366-3.129619-3.139284$ H $3.982072-3.870891-2.025666$ H $2.342406-4.547389-2.162321$ H $2.191161-2.8202981 .157311$ $\begin{array}{llll}\text { H } 2.191161 & -2.820298 & 1.157311\end{array}$ $\begin{array}{llll}\text { H } 3.606128 & -3.705212 & 0.530976\end{array}$ $\begin{array}{lllll}\text { H } 1.973753 & -4.368756 & 0.298765\end{array}$ H $5.140300-1.991179-0.944513$ H $6.332833-0.152570-0.582859$ H $5.070995-2.288246-0.600862$ $\begin{array}{llll}C & -1.272025 & -1.162218 & 1.441806\end{array}$ C $-1.017935-1.0818112 .929886$ C $-2.181639-1.730340 \quad 3.69928$ H $-2.241111-0.7111091 .190912$ H $-1.193368-2.189472 \quad 1.050364$ $\mathrm{H}-0.084862-1.597243 \quad 3.204659$ H $-0.928692 \quad-0.027238 \quad 3.238150$ H $-3.131901-1.224527 \quad 3.472734$ H $-2.288345-2.793143 \quad 3.433362$ H $-2.008947-1.665508 \quad 4.784793$ $\begin{array}{llll}\mathrm{N} & 1.226882 & -0.263650 & 1.395578\end{array}$ $\begin{array}{llll}\text { C } 2.066719 & -0.166805 & 2.194709\end{array}$ $\begin{array}{lll}\text { C } 3.066719 & -0.166805 & 2.194709 \\ \text { C } 3.029990 & -0.112367 & 3.224360\end{array}$ C 4.3429160 .0411162 .982806 $\begin{array}{lllll}\text { H } 2.636266 & -0.201487 & 4.241253\end{array}$ H $5.0523960 .069923 \quad 3.811956$ $\begin{array}{lllll}\text { H } & 4.724915 & 0.134641 & 1.963225\end{array}$ 82

$19 \mathrm{R}=$ CHCNEt $\mathrm{ET} / \mathrm{p}$

$\begin{array}{lllll}\text { Ni } & 0.035035 & 0.027615 & 0.022574\end{array}$ $\begin{array}{lllll}\mathrm{N} 2 & 0.052361 & -0.018383 & 0.116737\end{array}$ $\begin{array}{llll}\mathrm{N} & 0.439230 & 1.981006 & 0.126687\end{array}$ $\begin{array}{llll}\text { C } 1.682106 & 2.282143 & 0.307329\end{array}$ $\begin{array}{llll}\text { C } 2.616326 & 1.146948 & 0.098463\end{array}$ $\begin{array}{llll}\text { C }-0.343341 & -1.955125 & 0.248424\end{array}$ C $-0.494094-2.646085-0.997155$ N $-0.689793-3.194959-2.011825$ C $-1.517216-2.262831-1.190918$ $\mathrm{C}-1.517216-2.2628311 .190918$ C $0.619267-2.2446760 .703671$ $4.619267-2.2446760 .703671$ 1.045750 .727707 $\mathrm{H}-1.751771-4.364861 \quad 0.619462$

H $-0.660363 \quad-4.1066632 .001647$ H $-2.419904-3.943501 \quad 2.22033$ $\begin{array}{llll}\text { C }-1.634807 & 0.180511 & -1.117537\end{array}$ C $-0.492626 \quad 0.156725-1.927735$ H $-0.219302-0.751963-2.466751$ H $-0.058660 \quad 1.084720-2.306716$ H $-2.266236-0.707794-1.051931$ н $-2.106056 \quad 1.121937 \quad-0.819083$ C $2.773010-1.275706 \quad 0.091194$ C $-0.672396 \quad 2.8479420 .409215$

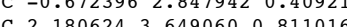
C $2.180624 \quad 3.6490600 .811016$ $\begin{array}{llll}\text { C } 4.086518 & 1.403195 & -0.270127\end{array}$ F $4.8664390 .321363-0.100299$ F $4.1192391 .759876-1.590605$ $\begin{array}{llll}F & 4.639994 & 2.408824 & 0.424173\end{array}$ F $3.132998 \quad 3.483072 \quad 1.738440$ $\begin{array}{llll}\text { F } 1.235685 & 4.403405 & 1.368848\end{array}$ $\begin{array}{llll}\text { F } 2.704957 & 4.344984 & -0.244134\end{array}$ C $-1.264897 \quad 2.649114 \quad 1.663908$ $\begin{array}{llll}\text { C }-1.185041 & 3.711734 & -0.576421\end{array}$ $\begin{array}{llll}\text { C }-2.415369 & 3.381377 & 1.947668\end{array}$ $\begin{array}{llll}\text { C } & -2.347806 & 4.417352 & -0.229557\end{array}$ C $-2.958700 \quad 4.263923 \quad 1.000958$ B $-0.617811 \quad 1.609232 \quad 2.720107$ F $0.722269 \quad 1.906631 \quad 3.034022$ F $-1.398257 \quad 1.399819 \quad 3.842972$ F $-0.5473220 .260096 \quad 1.993390$ $\begin{array}{llll}F & -0.547322 & 0.260096 & 1.993390\end{array}$ $\begin{array}{llll}C & -0.553152 & 3.910737 & -1.940134\end{array}$ $\begin{array}{llll}C & -0.052771 & 5.362171 & -2.123773\end{array}$ C $-1.5152653 .538825-3.079$ $\begin{array}{llll}\text { H } & 0.327989 & 3.253492 & -2.012420\end{array}$ $\begin{array}{lllll}\mathrm{H} & 0.640765 & 5.653542 & -1.320621 \\ \mathrm{H} & -0.899747 & 6.066874 & -2.115942\end{array}$ $\begin{array}{llll}\mathrm{H} & -0.899747 & 6.066874 & -2.115942\end{array}$ H $0.465600 \quad 5.462718-3.092191$ H $-2.386267 \quad 4.211947 \quad-3.089647$ н $-1.8900252 .509585-2.984900$ H $-1.007690 \quad 3.635798 \quad-4.051769$ H $-2.889192 \quad 3.253006 \quad 2.919235$ H $-3.863752 \quad 4.825258 \quad 1.237377$ H $-2.778262 \quad 5.106940-0.961209$ C $2.919331-1.892886-351392$ c $3.186448-1.882856-1.104343$ C $3.521628-3.1644691 .376875$ $\begin{array}{llll}\text { C } 3.762000 & -3.156987 & -1.012161\end{array}$ $\begin{array}{llll}\text { C } 3.762000 & -3.156987 & -1.012161 \\ \text { C } 3.927996 & -3.789178 & 0.215465\end{array}$ $\begin{array}{llll}\text { C } 2.524182-1.201091 & 2.642121\end{array}$ C $1.817062-2.1268093 .638106$ C $3.753232-0.5468833 .296011$ H $1.815340-0.3949432 .403229$ H $0.953795-2.630786 \quad 3.180171$ H $2.494574-2.897936 \quad 4.036777$ H $1.449638-1.533424 \quad 4.488209$ H $4.2617290 .147784 \quad 2.611652$ H $4.485271-1.315324 \quad 3.594090$ H $3.446693 \quad 0.015288 \quad 4.190605$ C $3.085069-1.235763-2.475255$ $\begin{array}{llll}\text { C } 4.495094 & -0.976397 & -3.078495\end{array}$ C $2.276005-2.081731-3.468285$ H $4.981761-1.934550-3.321955$ H $5.981761-1.934550-3.321955$ $4-151383-0.427583-2.392617$ H $4.396526-0.399798-4.011506$ 1. $2.808156-3.017204-3.702517$ $\begin{array}{lllll}\text { H } 1.286837 & -2.358664 & -3.082784\end{array}$ H $2.152318-1.529403-4.412656$ $\begin{array}{llll}\text { H } 3.659960 & -3.655887 & 2.342277\end{array}$ $\begin{array}{lllll}\text { H } 4.383787 & -4.785244 & 0.255236\end{array}$ $\begin{array}{rrrrr}\text { H } & 4.086897 & -3.655088 & -1.924746 \\ \text { H } & 2.580500 & -0.263733 & -2.364347\end{array}$ 83

$19 \mathrm{R}=\mathrm{CHCNEt} \mathrm{AC} / \mathrm{pi}$

Ni $0.052813-0.062447-0.017760$ N 2.071806 $-0.049353 \quad 0.009364$ N $0.417925-2.046768-0.003360$ $\begin{array}{llll}\text { c } 1.668919 & -2.367237 & -0.045461\end{array}$ $\begin{array}{llll}\text { C } 2.617409 & -1.228459 & 0.097915\end{array}$ C $-0.240907 \quad 1.896818-0.481880$ C $-1.6371272 .237310-1.014108$ $\begin{array}{llll}\text { C } & -1.637127 & 2.237310 & -1.014108 \\ \text { C } & -1.613860 & 3.547065 & -1.815174\end{array}$ C $0.153704 \quad 2.807753 \quad 0.548324$ N $0.3648423 .579149 \quad 1.403212$ H $0.4971801 .890162-1.296231$ $\begin{array}{lllll}\mathrm{H} & -2.338922 & 2.355867 & -0.174847\end{array}$ H $-1.9835761 .416018-1.65615$ H $-0.966414 \quad 3.455774 \quad-2.699095$ H $-1.2495884 .381948-1.19833$ H $-2.629621 \quad 3.788617 \quad-2.161543$ C $-1.690401 \quad-0.224732 \quad 0.965132$ $\begin{array}{llll}\text { C }-0.677884 & 0.183895 & 1.860812\end{array}$ $\begin{array}{lll}\text { C }-0.012726 & -0.690090 & 2.768949\end{array}$ 
F $4.337519-1.9992151 .535783$ $\begin{array}{llll}\text { F } 4.870907 & -0.357423 & 0.205630\end{array}$ $\begin{array}{llll}\text { F } & 4.598345 & -2.331452 & -0.630614\end{array}$ F $2.659175-3.873635-1.588919$ F $3.168689-4.093744 \quad 0.539618$ F $1.267932-4.756682-0.162088$ C $-1.067481-2.725818-1.742024$ $\begin{array}{llll}\text { C }-1.334728 & -3.770592 & 0.494001\end{array}$ C $-2.170341-3.459390-2.190800$ C -2.433652 $\begin{array}{llll}C & -2.433652 & -4.471424 & -0.012278\end{array}$ $\begin{array}{llll}C & -2.857518 & -4.327364 & -1.348855\end{array}$ B $-0.340673-1.654837-2.705957$ F $-0.471642-0.295787-1.974320$ F $1.041239-1.841122-2.844900$ $F-0.982125-1.478982-3.920426$ $\begin{array}{llll}C & -0.933701 & -3.966965 & 1.944470\end{array}$ $\begin{array}{llll}\text { C }-0.605356 & -5.457363 & 2.27319\end{array}$ $\begin{array}{llll}\text { C }-2.025356 & -3.478974 & 2.909223\end{array}$ H $-0.023870-3.3835412 .142433$ H $0.166105-5.865691 \quad 1.604817$ H $-0.246423 \quad-5.528745 \quad 3.313246$ H $-1.507855-6.084240 \quad 2.181706$ H $-2.295208-2.4263312 .743320$ H $-1.676043-3.573502 \quad 3.948043$ H $-2.940650-4.0813092 .796098$ H $-2.487377-3.322612-3.226148$ $\begin{array}{llll}\mathrm{H} & -2.487377 & -3.322612 & -3.226148 \\ \mathrm{H} & -3.720737 & -4.892613 & -1.710524\end{array}$ $\begin{array}{llll}\mathrm{H} & -3.720737 & -4.892613 & -1.710524 \\ \mathrm{H} & -2.975528 & -5.153002 & 0.646031\end{array}$ $\begin{array}{llll}\text { H } & -2.975528 & -5.153002 & 0.646031 \\ \text { C } 3.026406 & 1.856522 & -1.143407\end{array}$ $\begin{array}{llll}\text { C } 3.026406 & 1.856522 & -1.143407 \\ \text { C } 3.229570 & 1.719024 & 1.317144\end{array}$ $\begin{array}{llll}\text { C } 3.229570 & 1.719024 & 1.317144 \\ \text { C } 3.646125 & 3.106264 & -1.092943\end{array}$ $\begin{array}{llll}\text { C } 3.646125 & 3.106264 & -1.092943 \\ \text { C } 3.824038 & 2.982223 & 1.301088\end{array}$ $\begin{array}{llll}\text { C } & 3.824038 & 2.982223 & 1.301088 \\ \text { C } & 4.028112 & 3.676269 & 0.118208\end{array}$ $\begin{array}{llll}\text { C } & 4.028112 & 3.676269 & 0.118208 \\ \text { C } & 2.705686 & 1.199877 & -2.474297\end{array}$ $\begin{array}{llll}\text { C } & 2.705686 & 1.199877 & -2.474297 \\ \text { C } & 2.213196 & 2.198719 & -3.553396\end{array}$ $\begin{array}{llll}\text { C } 2.213196 & 2.198719 & -3.553396 \\ \text { C } 3.921910 & 0.426784 & -3.012874\end{array}$ $\begin{array}{llll}\text { C } 3.921910 & 0.426784 & -3.012874\end{array}$ н $1.9035910 .461562-2.322070$ H $1.4147862 .854575-3.173231$ H $1.821075 \quad 1.634065-4.413207$ H $3.0363262 .835221-3.916664$ H $4.258416-0.356068-2.321003$ H $3.660995-0.058362-3.964992$ H $4.7680561 .112320-3.180741$ $\begin{array}{llll}\mathrm{H} & 4.768056 & 1.112320 & -3.18074 \\ \mathrm{C} & 3.083792 & 1.003451 & 2.649767\end{array}$ $\begin{array}{llll}\text { C } & 3.083792 & 1.003451 & 2.649767 \\ \text { C } & 2.182329 & 1.771995 & 3.624408\end{array}$ $\begin{array}{llll}\text { C } & 2.182329 & 1.771995 & 3.624408 \\ \text { C } & .448516 & 0.774247 & 3.322646\end{array}$ $\begin{array}{llll}\text { C } & 4.448516 & 0.774247 & 3.322646 \\ \mathrm{H} & 2.627776 & 0.017190 & 2.483056\end{array}$ $\begin{array}{lllll}\text { H } & 2.627776 & 0.017190 & 2.483056 \\ \text { H } & 1.215573 & 2.037288 & 3.179639\end{array}$ $\begin{array}{llll}\mathrm{H} & 1.215573 & 2.037288 & 3.179639 \\ \mathrm{H} & 2.658530 & 2.717061 & 3.929880\end{array}$ $\begin{array}{llll}\mathrm{H} & 2.658530 & 2.717061 & 3.929880 \\ \mathrm{H} & 2.010684 & 1.166127 & 4.526427\end{array}$ $\begin{array}{llll}\text { H } & 2.010684 & 1.166127 & 4.526427 \\ \text { H } & 5.158820 & 0.256224 & 2.662996\end{array}$ $\begin{array}{llll}\mathrm{H} & 5.158820 & 0.256224 & 2.662996 \\ \mathrm{H} & 4.900988 & 1.733770 & 3.622671\end{array}$ H $4.900988 \quad 1.733770 \quad 3.622671$ H $4.3108850 .167554 \quad 4.232214$ $\begin{array}{llll}\text { H } & 3.834755 & 3.643194 & -2.024462\end{array}$ H $4.495269 \quad 4.663587 \quad 0.134500$ H

$19 \mathrm{R}=\mathrm{CHCNE} \mathrm{AC} / \mathrm{N}$

Ni $-0.165490-0.6052860 .195638$ $\begin{array}{llll}\mathrm{N} & 1.069766 & 0.880511 & 0.080995\end{array}$ $\begin{array}{llll}\mathrm{N} & 1.069766 & .880511 & 0.080995\end{array}$ $\begin{array}{llll}\text { C } 2.479622 & 0.648654 & -0.060265\end{array}$ $\begin{array}{llll}\text { C } & 2.479622 & 0.648654 & -0.060265 \\ \text { C } & 0.513860 & 2.065747 & 0.101870\end{array}$ $\begin{array}{llll}C & 0.513860 & 2.065747 & 0.101870 \\ C & 1.308623 & 3.373777 & -0.004125\end{array}$ $\begin{array}{llll}\text { F } & 1.308623 & 3.373777 & -0.004125 \\ \text { F } & 0.630835 & 3.894501 & 1.226882\end{array}$ $\begin{array}{llll}\text { F } & 1.530435 & 3.894501 & 1.226882 \\ \text { F } & 0.630844 & 4.287659 & -0.743293\end{array}$ F $0.630844 \quad 4.287659-0.743293$ F $2.5097913 .206141-0.592301$ $\begin{array}{lllll}\text { C } & -0.933129 & 2.012317 & 0.304998\end{array}$ C $-1.763020 \quad 3.282532 \quad 0.549790$ F $-1.083521 \quad 4.159028 \quad 1.320293$ F $-1.999572 \quad 3.875324 \quad-0.659268$ F $-2.950382 \quad 3.0703151 .125673$ C $-2.774774 \quad 0.521741 \quad 0.731529$ $\begin{array}{llll}\text { C }-2.804804 & 0.217245 & 2.104874\end{array}$ C $-3.8881220 .452109 \quad-0.117908$ $\begin{array}{llll}\text { C }-4.053906 & -0.095841 & 2.644594\end{array}$ C $-5.1127040 .145460 \quad 0.489029$ $\begin{array}{llll}\text { C } & -5.112704 & 0.145460 & 0.489029 \\ \text { C }-5.202268 & -0.112051 & 1.852975\end{array}$ B $-1.4484770 .159643 \quad 2.996445$ F $-1.680354-0.287810 \quad 4.292110$ $\begin{array}{lllll}F & -0.541571 & -0.849574 & 2.338715\end{array}$ $\begin{array}{llll}F & -0.735646 & 1.384942 & 2.980573\end{array}$ C $-3.8108400 .696301-1.614673$ C $-4.5206521 .999616-2.013246$ $\begin{array}{llll}\text { C }-4.394127 & -0.478472 & -2.415203\end{array}$ H $-2.745673 \quad 0.790097 \quad-1.885379$ H $-4.115062 \quad 2.867871-1.478012$ H $-4.4114242 .172983-3.094947$ H $-5.596528 \quad 1.934508-1.786285$ н $-3.925226-1.436304-2.149079$ H $-4.236645-0.314226-3.491802$ H $-5.478108-0.569368-2.244003$ H $-4.109662-0.327372 \quad 3.709574$ H $-6.172315-0.3394312 .297446$ $\begin{array}{llll}\text { H } & -6.172315 & -0.339431 & 2.297446 \\ \text { H } & -6.013227 & 0.105871 & -0.124878\end{array}$ $\begin{array}{llll}\text { H } & -6.013227 & 0.105871 & -0.124878\end{array}$ $\begin{array}{llll}\text { C } 2.975311 & 0.284321 & -1.327906\end{array}$ $\begin{array}{llll}\text { C } 4.637615 & 0.378624 & 0.936934\end{array}$ $\begin{array}{lllll}C & 4.340774 & 0.010717 & -1.428667\end{array}$ $\begin{array}{lllll}\text { C } 5.173708 & 0.067697 & -0.313880\end{array}$ C $2.719574 \quad 0.9499212 .478345$ $\begin{array}{llll}\text { C } 2.694457 & -0.329192 & 3.326549\end{array}$ C $3.506533 \quad 2.068914 \quad 3.213705$ H $1.678595 \quad 1.289047 \quad 2.387749$ H $2.084760-1.110918 \quad 2.851355$ н $3.714717-0.715894 \quad 3.484198$ H $2.248657-0.1137154 .308875$ $\begin{array}{llll}\text { H } 2.248657 & -0.113715 & 4.308875\end{array}$ H $3.5912762 .975175 \quad 2.594189$ $\begin{array}{llll}\text { H } & 4.522791 & 1.733279 & 3.479491\end{array}$ c $2.98708740 .196663-2.547541$ $\begin{array}{llll}\text { C } & 2.070874 & 0.196663 & -2.547541 \\ \text { C } 2.473755 & -0.926236 & -3.509720\end{array}$ $\begin{array}{llll}\text { C } & 2.473755 & -0.926236 & -3.509720 \\ C & 1.991802 & 1.530017 & -3.309970\end{array}$ $\begin{array}{llll}\text { C } 1.991802 & 1.530017 & -3.309970\end{array}$ H $1.055643-0.042237-2.19021$ H $1.5678642 .338451-2.700681$ H $2.995293 \quad 1.847753-3.635749$ H $1.358156 \quad 1.409118-4.201806$ н $2.626056-1.879622-2.982748$ H $3.396491-0.678675-4.057715$ H $1.673888-1.076524-4.248765$ H $5.288972 \quad 0.402839 \quad 1.812010$ H $6.242746-0.136881-0.415940$ H $4.759257-0.256316-2.400432$ C $-1.498532-2.006890-0.376974$ $\begin{array}{llll}C & -1.498532 & -2.006890 & -0.376974\end{array}$ $\begin{array}{llll}\text { C }-1.490613 & -3.282526 & 0.465129\end{array}$ C $-2.727571-4.1433600 .179217$ $\begin{array}{llll}C & -1.222046 & -2.184565 & -1.770939\end{array}$ $\mathrm{N}-0.964119-2.310735-2.907139$ H $-2.452688-1.478081-0.24058$ H $-1.472478-2.989214 \quad 1.525565$ $\begin{array}{llll}\mathrm{H}-0.581815 & -3.867415 & 0.264444\end{array}$ $\begin{array}{llll}\text { H } & -3.650570 & -3.600679 & 0.433049\end{array}$ H $-2.773186-4.429963-0.882877$ H $-2.695878-5.062760 \quad 0.783218$ N $1.100912-1.903242-0.028907$ $\begin{array}{llll}\text { C } 1.911936 & -2.719710 & -0.191797\end{array}$ $\begin{array}{llll}\text { C } 2.847761 & -3.751279 & -0.412080\end{array}$ $\begin{array}{llll}\text { C } 4.144885 & -3.624568 & -0.088379\end{array}$ H $2.449988-4.661589-0.868444$ H $4.839926-4.444614-0.278673$ $\begin{array}{llll}\text { H } & 4.839926 & -4.444614 & -0.278673 \\ \mathrm{H} & 4.531966 & -2.705765 & 0.358598\end{array}$

87

$20 \mathrm{R}=\mathrm{Me}$

Ni $-0.034571-0.062353-0.020327$ N $1.818808-0.006152-0.01546$ C $2.488320 \quad 1.273154 \quad 0.003723$

$\begin{array}{lll}\text { C } 2.426402 & -1.182666 & 0.022200\end{array}$ $\begin{array}{llll}\text { C } 3.878878 & -1.398286 & 0.550132\end{array}$ $\begin{array}{llll}\text { C } 4.004106 & -0.658534 & 1.902656\end{array}$ $\begin{array}{llll}\text { C } 4.998953 & -0.878888 & -0.372626\end{array}$ $\begin{array}{lllll}\text { C } 4.174655 & -2.872201 & 0.878596\end{array}$ $\begin{array}{llll}\text { C } 1.439630 & -2.315573 & -0.169646\end{array}$ $\begin{array}{llll}\text { C } 1.666715 & -3.508081 & -1.151215\end{array}$ $\begin{array}{lll}\text { C } 2.778292 & -3.126685 & -2.153221\end{array}$ C $1.949454-4.892352-0.530567$ C $0.393685-3.675074-2.018909$ C $0.393685-3.675074-2.018909$ C $0.270283-1.981623-0.333199$ C $-0.788801-2.9139940 .588318$ C $-2.047397-2.6993080 .006741$ C $-0.569715-3.9414251 .544362$ C $-3.047972-3.643073 \quad 0.254269$ $\begin{array}{llll}C & -1.606273 & -4.856188 & 1.74462\end{array}$ C $-2.823010-4.7411981 .077002$ B $-2.407265-1.373685-0.826567$ F $-1.687740-1.187484-2.018553$ F $-3.764851-1.177293-0.994980$ F $-1.923874-0.2203010 .108403$ C $0.642578-3.951247 \quad 2.464462$ $\begin{array}{llll}\text { C } 0.920175 & -5.300851 & 3.129915\end{array}$ C $0.474936-2.854214 \quad 3.531113$ H $1.532446-3.6994361 .872921$ H $1.532446-3.6994361 .872921$ $\begin{array}{lll}0.996900-6.115887 & 2.394777\end{array}$ $\begin{array}{llll}H & 1.868466 & -5.250477 & 3.686612\end{array}$ $0.133979-5.5618393 .854796$ 1. $0.344825-1.8662603 .065100$ $\mathrm{H}-0.419770-3$. $\begin{array}{llll}H & 1.352534 & -2.821998 & 4.196654\end{array}$ H $-4.026108-3.487203-0.202711$ $\mathrm{H}-3.606472-5.4819251 .242396$ H $-1.467977 \quad-5.668776 \quad 2.456670$ C $3.0935601 .727860-1.191199$ C $2.3666362 .114033 \quad 1.131003$ C $3.6032663 .031245-1.211462$ $\begin{array}{llll}\text { C } 2.909774 & 3.398671 & 1.058597\end{array}$ $\begin{array}{llll}\text { C } 3.523622 & 3.863762 & -0.098047\end{array}$ $\begin{array}{llll}\text { C } 3.152450 & 0.895171 & -2.463214\end{array}$ $\begin{array}{llll}\text { C } 4.457769 & 1.127423 & -3.287081\end{array}$ C $1.9312131 .127823-3.287081$ c $1.9312131 .138679-3.362535$ H $3.141659-0.164217-2.179456$ H $4.4453152 .110791-3.785066$ H. $5.3613171 .065788-2.659495$ H $1.0000040 .831964 \quad-2.871443$

H $2.033934 \quad 0.563855 \quad-4.296491$ H $1.8476832 .206205 \quad-3.621934$ C $1.651832 \quad 1.7012992 .411215$ C $0.317410 \quad 2.449294 \quad 2.567108$ C $2.506647 \quad 1.9322053 .668379$ $\begin{array}{lllll}\text { H } & 1.423997 & 0.626920 & 2.342310\end{array}$ H $-0.354319 \quad 2.256422 \quad 1.722248$ H $0.488060 \quad 3.535883 \quad 2.630105$ $\mathrm{H}-0.187012 \quad 2.126793 \quad 3.491067$ H $3.499863 \quad 1.4681633 .598339$ H 2.6494633 .0076473 .855890 H 2.6494633 .0076473 .855890 H 1.9906491 .5146954 .546579 H $4.0742753 .403576-2.123222$ H $3.9338954 .875763-0.138011$ H 2.8377734 .0536081 .928323 $\begin{array}{llll}\text { C }-0.421991 & 1.645369 & -0.836636\end{array}$ H $-1.21124220 .104494-0.221965$ H $-0.843870 \quad 1.366297-1.81499$ H $0.454468 \quad 2.298227 \quad-0.933605$ H $4.968897-0.933213 \quad 2.357491$ H $3.994959 \quad 0.428041 \quad 1.774683$ H $3.202421 \quad-0.952893 \quad 2.596725$ H $5.966065-1.1344250 .090543$ H $4.973473-1.338472-1.370424$ H $4.9588320 .212243-0.481429$ H $5.136356-2.909707-1.412890$ H $3.413922-3.3014691 .546277$ H $3.413922-3.3014691 .546277$ $\begin{array}{llll}\text { H } & 4.273813 & -3.508476 & -0.005522\end{array}$ $2.873506-3.938560-2.890824$ H $2.502981-2.212783-2.702935$ H $3.763592-2.980079-1.700385$ H $0.656578-4.316267-2.874883$ H $-0.422438-4.166961-1.475699$ H $0.022534-2.713616-2.399748$ H $2.086652-5.608384-1.357393$ $\begin{array}{llll}\text { H } 2.845553 & -4.932453 & 0.098978\end{array}$ $\mathrm{H}$
93

$20 \mathrm{R}=\mathrm{Me} \mathrm{ET} / \mathrm{pi}$

Ni $0.137453-0.089816-0.025943$ $\begin{array}{llll}\mathrm{N} & 2.158501 & -0.087842 & 0.051359\end{array}$ N $0.535866-2.046378-0.002870$ $\begin{array}{llll}\text { C } 1.779543 & -2.414907 & -0.068233\end{array}$ $\begin{array}{llll}\text { C } 2.752948 & -1.243254 & 0.235857\end{array}$ $\begin{array}{llrr}\text { C } & 2.752948 & -1.243254 & 0.235857 \\ \text { C } & -0.228915 & 1.792853 & -0.381226\end{array}$ $\begin{array}{lllll}\text { C } & -0.228915 & 1.792853 & -0.381226 \\ \mathrm{H} & 0.725165 & 2.255393 & -0.668585\end{array}$ $\mathrm{H}-0.923406 \quad 1.738997-1.232287$ $\begin{array}{llll}\mathrm{H} & -0.691123 & 2.326206 & 0.458899\end{array}$ C $-1.673479-0.136564 \quad 0.842100$ $\begin{array}{llll}\text { C }-0.651112 & 0.032663 & 1.794022\end{array}$ H $-0.279412-0.819250 \quad 2.370680$ H $-2.106723-1.120802 \quad 0.643493$ H $-2.294268 \quad 0.712192 \quad 0.548132$ H $-0.484978 \quad 1.010199 \quad 2.249815$ B $-0.378647-1.634251-2.741025$ F $-0.196315-0.305637-2.083249$ F $0.926898-2.067995-3.089197$ F $-1.153719-1.422855-3.880294$ C $2.8091291 .188500-0.105481$ C $-0.612162-2.836888-0.374678$ $\begin{array}{llll}\text { C }-0.612162 & -2.836888 & -0.374678\end{array}$ $\begin{array}{llll}\text { C }-1.101480 & -2.624400 & -1.680466\end{array}$ $\begin{array}{llll}C & 2.286330 & -3.791671 & -0.560603\end{array}$ C $5.033017-0.1414190 .950027$ C $5.033017-0.1414190 .950027$ $\begin{array}{llll}C & 5.154017 & -2.484432 & 0.465770\end{array}$ $\begin{array}{lll}\text { C } 3.749000 & -1.696386 & 2.421385\end{array}$ $\begin{array}{lll}\text { C } 3.340996 & -3.530059 & -1.674321\end{array}$ $\begin{array}{llll}\text { C } 2.773304 & -4.642072 & 0.643674\end{array}$ C $1.260296-4.710731-1.263980$ $\begin{array}{llll}\text { C }-1.283167 & -3.643271 & 0.563251\end{array}$ $\begin{array}{llll}\text { C }-2.253664 & -3.316556 & -2.056220\end{array}$ $\begin{array}{llll}\text { C }-2.437530 & -4.307912 & 0.124722\end{array}$ $\begin{array}{llll}\text { C } & -2.914971 & -4.168600 & -1.173515\end{array}$ C $-0.823044-3.843106 \quad 2.000184$ $\begin{array}{llll}\text { C }-0.612352 & -5.328827 & 2.337970\end{array}$ C $-1.815351-3.2399123 .009476$ C $-1.815351-3.2399123 .009476$ $\begin{array}{llll}\mathrm{H} & 0.142222 & -3.326182 & 2.122690\end{array}$ $+0.034646-5.8353431 .608171$ H $-0.161270-5.4337183 .337257$ $\mathrm{H}-1.574065-5.8649812 .351209$ $\mathrm{H}-1.989613-2.1705172 .832058$ H $-1.438492-3.366884 \quad 4.037168$ H $-2.788763-3.751382 \quad 2.942892$ H $-2.630164-3.169107-3.069853$ H $-3.811163-4.705842-1.486657$ н $-2.970794 \quad-4.954993 \quad 0.823020$ C $3.232187 \quad 1.500471-1.417521$ $\begin{array}{llll}\text { C } 2.870998 & 2.154784 & 0.922418\end{array}$ $\begin{array}{llll}\text { C } 3.794973 & 2.758853 & -1.647791\end{array}$ $\begin{array}{llll}\text { C } 3.444205 & 3.394868 & 0.633682\end{array}$ C $3.9179683 .702375-0.636062$ 
H $2.818916 \quad 2.024775-4.195558$ H $5.024614-0.439133-2.30286$ H $4.309607-0.657554-3.92129$ $\begin{array}{lllll}\text { H } 5.056409 & 0.898747 & -3.471878\end{array}$ $\begin{array}{llll}\text { C } 2.346348 & 1.926689 & 2.336857\end{array}$ $\begin{array}{llll}\text { C } 1.266182 & 2.957222 & 2.702631\end{array}$ C $3.4367842 .002151 \quad 3.422027$ H $1.8900390 .925977 \quad 2.367493$ H $0.494023 \quad 3.038648 \quad 1.928912$ $\mathrm{H} 1.7128153 .9$ $\begin{array}{llll}H & 1.712815 & 3.955968 & 2.83108\end{array}$

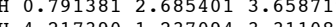
$\begin{array}{llll}H & 4.217390 & 1.237094 & 3.311082\end{array}$ $\begin{array}{ll}4.9300412 .988089 & 3.408291\end{array}$ H $2.978131 \quad 1.872165 \quad 4.416192$ $\begin{array}{lllll}\text { H } & 4.131776 & 3.005739 & -2.656949\end{array}$ $\begin{array}{lllll}\text { H } & 4.359835 & 4.679499 & -0.843605\end{array}$ H $3.511967 \quad 4.142756 \quad 1.426530$ H $3.434603 \quad-4.139488 \quad 1.352267$ H $3.296460-5.532860 \quad 0.259321$ н $1.897867-4.990814 \quad 1.209440$ H $4.019995-4.393456-1.758195$ H $2.807675-3.400562-2.625844$ H $3.944628-2.630076-1.531693$ H $1.836925-5.583040-1.616216$ H $0.468099-5.083506-0.604914$ H $0.811059-4.231101-2.139799$ $\begin{array}{llll}\mathrm{H} & 0.811059 & -4.231101 & -2.139799\end{array}$ $\begin{array}{llll}H & 4.769197 & -3.490510 & 0.323492\end{array}$ $\begin{array}{llll}\text { H } 5.939319 & -2.540427 & 1.235998\end{array}$ $\begin{array}{llll}\text { H } & 5.642111 & -2.168674 & -0.46787 \\ \text { H } & 4.664176 & -1.799270 & 3.026875\end{array}$ $\begin{array}{llll}\text { H } & 4.664176 & -1.799270 & 3.026875 \\ \text { H } 3.155123 & -0.867314 & 2.832194\end{array}$ H $3.155123-0.8673142 .832194$ H $3.160603-2.6179662 .532373$ н $5.970606 \quad-0.405332 \quad 1.464603$ $\begin{array}{lllll}\text { H } & 5.291966 & 0.184157 & -0.067138\end{array}$ $\begin{array}{llll}\text { H } & 4.601536 & 0.707064 & 1.477333\end{array}$

94

$20 \mathrm{R}=\mathrm{Me} \mathrm{AC} / \mathrm{pi}$

Ni $0.124479-0.076123 \quad 0.001586$ $\begin{array}{llll}\mathrm{N} 2 & 2.166085 & -0.070783 & 0.052449\end{array}$ N $0.511553 \quad-2.053693 \quad 0.037774$ $\begin{array}{llll}\text { C } 1.756353 & -2.407168 & -0.045123\end{array}$ $\begin{array}{llll}\text { C } 2.742802 & -1.229386 & 0.257160\end{array}$ $\begin{array}{llll}\text { C }-0.245502 & 1.803058 & -0.414473\end{array}$ H $0.726805 \quad 2.279756-0.598253$ $\begin{array}{lllll} & -0.809207 & 2.337166 & 0.360984\end{array}$ C $-1.639866-0.163950 \quad 0.895704$ C $-0.626840 \quad 0.213866 \quad 1.819570$ C $-0.028589-0.643474 \quad 2.788579$ N $0.517451-1.273585 \quad 3.608275$ $\mathrm{H}-2.013792 \quad-1.189683 \quad 0.85070$ $\begin{array}{llll}\mathrm{H} & -2.325712 & 0.607938 & 0.542245\end{array}$ H $-0.562872 \quad 1.264372 \quad 2.114132$ B $-0.375147-1.633396-2.708978$ F $-0.176177-0.312282-2.016083$ F $0.925189-2.059573-3.071638$ F $-1.149958-1.374900-3.836182$ C $2.833954 \quad 1.201800-0.118211$ C $-0.627576-2.859728-0.357455$ C $-1.101822-2.632254-1.667064$ C $2.274160-3.775117-0.548956$ $\begin{array}{llll}\text { C } & 2.274160 & -3.775117 & -0.548956\end{array}$ $\begin{array}{llll}C & .141027 & -1.402588 & 0.962325\end{array}$ $\begin{array}{llll}\text { C } 5.043301 & -0.150187 & 0.937090\end{array}$ $\begin{array}{llll}\text { C } 5.128743 & -2.505396 & 0.500552 \\ \text { C } 3.742676-1.660291 & 2.443202\end{array}$ $\begin{array}{llll}\text { C } 3.742676 & -1.660291 & 2.443202 \\ \text { C } 3.336649 & -3.495062 & -1.651135\end{array}$ $\begin{array}{llll}\text { C } & 3.336649 & -3.495062 & -1.651135 \\ \text { C } 2.752188 & -4.624655 & 0.661517\end{array}$ $\begin{array}{llll}\text { C } & 2.752188 & -4.624655 & 0.661517 \\ \text { C } & 1.267744 & -4.702877 & -1.270620\end{array}$ $\begin{array}{llll}\text { C } & 1.267744 & -4.702877 & -1.270620 \\ \text { C } & -1.295101 & -3.707894 & 0.547828\end{array}$ $\begin{array}{llll}\text { C } & -2.238457 & -3.331345 & -2.077012\end{array}$ $\begin{array}{llll}\text { C }-2.432684 & -4.378057 & 0.073610\end{array}$ $\begin{array}{llll}\text { C }-2.900318 & -4.211010 & -1.223545\end{array}$ C $-0.860887-3.960552 \quad 1.983452$ $\begin{array}{llll}\text { C }-0.614986 & -5.454500 & 2.258936\end{array}$ C $-1.903051-3.4415702 .988766$ C $0.077716-3.417478 \quad 2.166095$ $\begin{array}{lll}\text { H } 0.077716 & -3.417478 & 2.166095\end{array}$ H $0.054835-5.9149631 .519178$ $\mathrm{H}-0.172351-5.584982-3.258789$ $\mathrm{H}-1.560856-6.0183152 .238878$ $\mathrm{H}-2.127543-2.3761452 .844492$ $\mathrm{H}-1.531818-3.567886-4.017150$ H $-2.846576-4.001559 \quad 2.888453$ H $-2.599925-3.169412-3.093738$ H $-3.784400-4.754044-1.560579$ H $-2.959304-5.0552850 .74752$ C $3.2787221 .492890-1.428931$ $\begin{array}{llll}\text { C } 2.903638 & 2.181559 & 0.895829\end{array}$ $\begin{array}{llll}\text { C } 3.890630 & 2.729273 & -1.659719\end{array}$ $\begin{array}{llll}\text { C } 3.508207 & 3.414221 & 0.600927\end{array}$ C $4.019553 \quad 3.687947-0.658281$ C $3.0710110 .549228-2.605609$ C $2.2167951 .216096-3.695424$ C $2.2167950 .216096-3.695424$ $\begin{array}{llll}\text { C } & 4.393657 & 0.040920 & -3.196354\end{array}$ H $2.500910-0.323636-2.267196$ H $1.2695231 .585336-3.279757$ $\begin{array}{llll}\text { H } & 2.748222 & 2.059914 & -4.163973\end{array}$
H $5.011054-0.473481-2.443993$ 01044 H $4.9921650 .867008 \quad-3.613148$ C $2.3504891 .990835 \quad 2.301054$ C $1.2908203 .058936 \quad 2.613403$ C 3.4163332 .0548193 .408902 H $1.878497 \quad 1.000021 \quad 2.348491$ H $0.547088 \quad 3.150449 \quad 1.811710$ H $1.764205 \quad 4.046757 \quad 2.729612$ H 0.7802892 .8 H 0.7802892 .8267693 .56113 $\begin{array}{llll}\mathrm{H} & 4.183120 & 1.273322 & 3.31451\end{array}$ H 3.9272753 .0318823 .405828 . 2.9310191 .9288314 .390633 H $4.2641632 .945589-2.663426$ H $4.5176074 .637249-0.864979$ H 3.5931784 .1610261 .392597 H $3.345852 \quad-4.098930 \quad 1.411907$ H $3.341390-5.4779520 .287913$ H $1.871160-5.0288881 .178140$ H $4.032292-4.345536-1.72655$ н $2.812208-3.376470-2.608771$ H $3.923109-2.584262-1.503422$ H $1.861458-5.566914-1.615427$ H $0.471364-5.088793-0.625212$ H $0.826242-4.227819-2.152755$ H $4.726595-3.506172 \quad 0.371986$ $\begin{array}{ll}H & 0.726595-3.506172-0.371986\end{array}$ H $5.631162-2.213245-0.433080$ $\begin{array}{llll}\text { H } 5.631162 & -2.213245 & -0.433080\end{array}$ $1.663537-1.7595093 .040656$ H $3.151585-0.8278082 .846631$ H $3.143042-2.5703072 .579937$ H $5.983486-0.424677 \quad 1.440800$ $\begin{array}{llll}\text { H } & 5.293798 & 0.159447 & -0.087405\end{array}$ H $4.633145 \quad 0.709819 \quad 1.464466$

94

$20 \mathrm{R}=\mathrm{Me} \mathrm{AC} / \mathrm{N}$

Ni $0.000721-0.036256 \quad 0.048464$ $\begin{array}{llll}\mathrm{N} & 1.890548 & -0.017045 & 0.121553\end{array}$ $\begin{array}{llll}\mathrm{N} & 0.377056 & -1.919365 & -0.219980\end{array}$ C $1.610488-2.298315-0.425143$ $\begin{array}{llll}\text { C } 2.567570 & -1.143961 & -0.042186\end{array}$ C $-0.348138 \quad 1.904983 \quad 0.128202$ H 0.567423 $\begin{array}{llll}\mathrm{H} & 0.567423 & 2.458757 & -0.104129\end{array}$ $\mathrm{H}-1.12338922 .075255 \quad-0.633254$ $\mathrm{H}-0.7242592 .1668821 .128137$ $\mathrm{C}-1.757188-0.1349300 .508144$ C $-2.913819-0.1240700 .638963$ $\begin{array}{llll}C & -4.313432 & -0.069021 & 0.811121\end{array}$ C $-5.151035-0.9495700 .242613$ $\begin{array}{llll}\text { H } & -4.683126 & 0.757164 & 1.424600\end{array}$ H $-6.226226-0.854971 \quad 0.390197$ H $-4.775763-1.764553-0.377339$ B $-0.800631-1.330563-2.841735$ F $-0.729420-0.017187-2.202882$ F $0.529735-1.668446-3.226056$ F $-1.630283-1.236450-3.969125$ $\begin{array}{llll}\text { C } 2.500701 & 1.241388 & 0.442541\end{array}$ C $-0.821843-2.652204-0.509941$ C $-1.423776-2.388589-1.755705$ $\begin{array}{llll}\text { C } & 1.984012 & -3.728608 & -0.953356\end{array}$ C $4.115188-1.2591660 .157416$ $\begin{array}{llll}\text { C } 4.115188 & -1.259166 & 0.157416 \\ \text { C } 4.856033 & -1.103719 & -1.239513\end{array}$ $\begin{array}{llll}\text { C } 4.856990 & -2.576460 & 0.864699\end{array}$ $\begin{array}{llll}\text { C } 4.805940 & -0.207055 & 1.066764\end{array}$ $\begin{array}{llll}\text { C } 2.169020 & -4.695858 & 0.251030\end{array}$ $\begin{array}{llll}\text { C } 3.204903 & -3.715677-1.90098\end{array}$ $\begin{array}{llll}\text { C } 0.905858 & -4.406263 & -1.843111\end{array}$ $\begin{array}{llll}\text { C }-1.415421 & -3.442625 & 0.491206\end{array}$ $\begin{array}{llll}\text { C } & -2.622019 & -3.057358 & -2.021551\end{array}$ $\begin{array}{llll}\text { C }-2.619901 & -4.077482 & 0.163798\end{array}$ $\begin{array}{llll}C & -3.210066 & -3.909450 & -1.086210\end{array}$ C $-0.812494-3.604696 \quad 1.879469$ $\begin{array}{llll}\text { C }-0.946839 & -5.030462 & 2.430665\end{array}$ C $-1.419523-2.604282 \quad 2.877948$ H $0.260916-3.3742801 .808229$ H $-0.602729-5.786767 \quad 1.709756$ H $-0.602729-5.786767-1.709756$ $\mathrm{H}-0.354591-5.134025 \quad 3.353106$ $\mathrm{H}-1.992008-5.2625852 .687567$ $\mathrm{H}-1.226114-1.5673522 .572026$ $\mathrm{H}-0.988287-2.7582923 .880501$ H $-2.510941-2.7445902 .947105$ H $-3.089065-2.894255-2.995193$ H $-4.133342-4.441370-1.32494$ $\begin{array}{lllll}\text { H } & -3.097544 & -4.727299 & 0.89802\end{array}$ C $3.0407252 .034077-0.591542$ C $2.395267 \quad 1.7114751 .770961$ $\begin{array}{llll}\text { C } 3.614591 & 3.258518 & -0.229901\end{array}$ $\begin{array}{llll}\text { C } 3.006111 & 2.926095 & 2.078593\end{array}$ C $3.6382563 .688905 \quad 1.091641$ $\begin{array}{llll}\text { C } 2.964646 & 1.649129 & -2.061189\end{array}$ $\begin{array}{llll}\text { C } 1.720074 & 2.245952 & -2.740272\end{array}$ $\begin{array}{llll}\text { C } 4.232758 & 2.084994 & -2.870896\end{array}$ $\begin{array}{llll}C & .232758 & 2.084994 & -2.870896\end{array}$ $0.7916581 .817282-2.138709$
0.345144 $\begin{array}{llll}4 & 0.791658 & 1.817282 & -2.345144\end{array}$ $1.6986093 .340625-2.613434$ $\begin{array}{llll}\text { H } & 1.748247 & 2.019226 & -3.817224 \\ \mathrm{H} & 5.173147 & 1.827524 & -2.356877\end{array}$

H $4.227228 \quad 3.172970 \quad-3.051268$ H $4.220116 \quad 1.587670-3.854519$ C $1.571905 \quad 0.973560 \quad 2.820913$ $\begin{array}{llll}\text { C } 0.942479 & 1.925417 \quad 3.844518\end{array}$ $\begin{array}{llll}\text { C } 2.314553 & -0.151728 & 3.554851\end{array}$ H $0.741786 \quad 0.497859 \quad 2.266708$ H $2.615488-0.956824 \quad 2.871444$ H $3.213027 \quad 0.232242 \quad 4.065395$ H $1.653102 \quad-0.593971 \quad 4.316752$ $\begin{array}{llll}1 & 0.443218 & 2.773768 & 3.354027\end{array}$

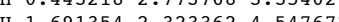
$\begin{array}{lllll}\mathrm{H} & 1.691354 & 2.323362 & 4.547672\end{array}$ 1.1956071 .3798904 .441188 H $4.0546863 .887358-1.005091$ H 4.1121174 .6386971 .352689 H 2.9680633 .3046403 .099621 H $5.867451-0.503923 \quad 1.111204$ H 4.7802060 .8167790 .681323 H $4.420199 \quad-0.213325 \quad 2.093707$ H $5.796494-1.681064-1.213539$ H $4.263136-1.434449-2.100243$ H $5.107480-0.045353-1.391182$ H $5.588784-2.567465 \quad 1.026749$ H $4.024827 \quad-2.630692 \quad 1.857597$ H $4.264113-3.487694 \quad 0.320319$ H $3.349544-4.743171-2.267716$ H $2.981718-3.083353-2.774192$ \begin{tabular}{l}
$4.981718-3.083353-2.774192$ \\
\hline
\end{tabular} H $2.769876-5.561290-0.074034$ $\begin{array}{lll}H & 2.769876-5.561290 & -0.074034\end{array}$ $1.188008-5.2569031 .130948$ $\begin{array}{llll}\mathrm{H} & 1.188008 & -5.074724 & 0.566315 \\ \mathrm{H} & 1.353021 & -5.352536 & -2.188023\end{array}$ $\begin{array}{lllll}\text { H } & 1.353021 & -5.352536 & -2.188023 \\ \mathrm{H} & -0.013087 & -4.665096 & -1.309556\end{array}$ $\begin{array}{llll}\text { H } & -0.013087 & -4.665096 & -1.309556 \\ \text { H } & 0.664074 & -3.792688 & -2.719215\end{array}$ 99 $20 \mathrm{R}=\mathrm{n}-\mathrm{Pr} \mathrm{ET} / \mathrm{pi}$

Ni $0.015836-0.050070 \quad 0.076936$ $\begin{array}{llll}\text { N } 2.044209 & -0.048156 & 0.082309\end{array}$ C $2.656878 \quad 1.244437 \quad 0.226885$ $\begin{array}{llll}\text { C } 2.666108 & -1.196221 & -0.047771\end{array}$ $\begin{array}{llll}\text { C } 4.128416 & -1.474672 & 0.459456\end{array}$ C $1.678693-2.312595-0.480685$ C $2.131248-3.547065-1.304496$ N $0.449805-2.001071-0.197496$ C $0.790103-2.773947-0.521219$ $\begin{array}{llll}C & -0.720103 & -2.773947 & -0.521219\end{array}$ $\begin{array}{llll}C & -1.423022 & -2.401613 & -1.686276\end{array}$ $\begin{array}{llll}C & -2.617042 & -3.076599 & -1.950794\end{array}$ C $-3.109307-4.061769-1.095746$ $\begin{array}{llll}\text { C }-2.408297 & -4.374756 & 0.061638\end{array}$ $\begin{array}{lllll}C & -1.204684 & -3.735283 & 0.385590\end{array}$ H $-3.167390-2.807464-2.853956$ H $-4.042602-4.578428-1.324621$ H $-2.801027-5.1374710 .735932$ B $-0.904154-1.276372-2.739456$ F $-0.375503-0.100492-1.979440$ F $0.187954-1.720279-3.525728$ $\begin{array}{llll}F & -1.941611 & -0.811492 & -3.549473\end{array}$ C $-0.497657-4.114878 \quad 1.677532$ C $-0.209374 \quad-5.623201 \quad 1.757183$ C $-1.310328 \quad-3.691296 \quad 2.912614$ C $-1.310328-3.691296 \quad 2.912614$ $\begin{array}{lllll}\text { H } & 0.299489 & -5.997073 & 0.857644\end{array}$ $\begin{array}{llll}\mathrm{H} & -1.144460 & -6.194532 & 1.865429\end{array}$ $\begin{array}{llll}\text { H } & 0.419264 & -5.847603 & 2.633298\end{array}$ $\mathrm{H}-2.264876-4.239915 \quad 2.950110$ H $-0.753478-3.9193273 .835631$ H $-1.546840-2.618795 \quad 2.90145$ $\begin{array}{llll}\text { H } & 0.465700 & -3.582369 & 1.708290\end{array}$ C $2.9894531 .891333-0.987365$ $\begin{array}{llll}\text { C } 3.556385 & 3.173961 & -0.926353\end{array}$ $\begin{array}{llll}\text { C } 3.747392 & 3.825061 & 0.284699\end{array}$ C $3.345843 \quad 3.189519 \quad 1.470100$ C $2.7916301 .907132 \quad 1.474324$ H $3.840054 \quad 3.668791-1.857414$ H $4.190083 \quad 4.823346 \quad 0.318037$ H $3.481637 \quad 3.7115592 .419716$ $\begin{array}{llll}\text { C } 2.767458 & 1.249256 & -2.352149\end{array}$ $\begin{array}{llll}\text { C } 2.009806 & 2.172069 & -3.318552\end{array}$ $\begin{array}{llll}\text { C } 4.092219 & 0.802329 & -2.989937\end{array}$ $\begin{array}{llll}\text { H } & 2.136289 & 0.360796 & -2.220868\end{array}$ $\begin{array}{llll}H & 4.753730 & 1.665568 & -3.170992\end{array}$ H $3.8979160 .309677-3.955186$ H $4.638050 \quad 0.093595-2.350612$ H $2.6029053 .060349-3.586930$ H $1.785855 \quad 1.621244 \quad-4.244258$ H $1.053998 \quad 2.503778 \quad-2.890120$ C $2.442741 \quad 1.2951862 .834652$ C $3.682877 \quad 0.999183 \quad 3.706270$ C $1.536513 \quad 2.207531 \quad 3.681704$ H $1.905282 \quad 0.352641 \quad 2.652093$ H $4.345923 \quad 0.232711 \quad 3.290920$ 
H $3.347176-3.094254 \quad 1.767477$ C 5.000994 H $5.180803 \quad 0.326027-0.252790$ H $5.976907-0.571150 \quad 1.055221$ H $4.611931 \quad 0.486331 \quad 1.412081$ C $5.085296-2.355365-0.386173$ H $4.701037-3.307116-0.740748$ H $5.457711-1.793223-1.25531$ H $5.955587-2.570083 \quad 0.254076$ C $2.998326-3.006496-2.477922$ $\begin{array}{llll}\text { C } 2.998326 & -3.006496 & -2.477922\end{array}$ $\begin{array}{llll}\text { H } 2.320407 & -2.668672 & -3.274774\end{array}$ H $3.638086-3.812704-2.871697$ H $3.636904-2.156502-2.223015$ $\begin{array}{llll}C & 2.808435 & -4.612902-0.400978\end{array}$ H $3.254860-5.384265-1.049306$ H $2.036168-5.1024010 .208814$ $\begin{array}{lllll}\text { H } 3.581910 & -4.257831 & 0.280928\end{array}$ C $1.027412-4.355691-2.027613$ H $0.451972-3.739679-2.725316$ H $1.561972-5.118414-2.619315$ н $0.345938-4.882980-1.349673$ C $-0.413332 \quad 1.871186-0.20467$ C $-0.758060 \quad 2.884383 \quad 0.859687$ H $-1.240383 \quad 1.733282-0.919170$ H $0.5003292 .165546-0.743656$ C $-1.738487-0.230250 \quad 1.036022$ C $-0.664224-0.114592 \quad 1.936273$

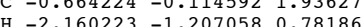
$\mathrm{H}-2.160223-1.207058 \quad 0.781862$

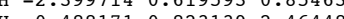
$\mathrm{H}-0.4881710 .8231392 .464482$ $\mathrm{H}-0.240627-1.005013 \quad 2.409637$ $\begin{array}{llll}C & -1.195281 & 4.220922 & 0.228657\end{array}$ $\begin{array}{llll}\text { H } & 0.120593 & 3.083855 & 1.487478\end{array}$ $\mathrm{H}-1.5705992 .531843 \quad 1.518323$ $\mathrm{H}-1.4351834 .956148 \quad 1.014800$ H $-0.389794 \quad 4.637349-0.396818$ 100

$20 \mathrm{R}=\mathrm{n}-\mathrm{Pr} \mathrm{AC} / \mathrm{pi}$

Ni $0.158639-0.142807 \quad 0.099345$ N $2.186271-0.164520 \quad 0.036391$

$\begin{array}{llll}\text { N } 0.532984 & -2.123998 & -0.021765\end{array}$

$\begin{array}{llll}\text { C } 1.762165 & -2.482213 & -0.239197\end{array}$ $\begin{array}{llll}\text { C } 2.775725 & -1.336202 & 0.068098\end{array}$ C $-0.0424901 .809031-0.199841$ C $-1.408165 \quad 2.456507-0.218426$ C $-1.2938713 .932507-0.638205$ $\begin{array}{llll}\text { H } & 0.633058 & 2.296666 & 0.514826\end{array}$ H $0.413848 \quad 1.815375-1.201697$ $\begin{array}{llll}\text { H } & -1.884619 & 2.425548 & 0.776397\end{array}$ H $-2.070518 \quad 1.932172 \quad-0.925678$ $\mathrm{H}-0.864504 \quad 4.022181-1.646659$ H $-0.646987 \quad 4.4911330 .055226$ H $-2.286400 \quad 4.408533-0.643323$ C $-1.578602 \quad-0.328804 \quad 1.056941$ $\begin{array}{llll}C & -0.567572 & 0.105538 & 1.948225\end{array}$ $\begin{array}{llll}\text { C } 0.103664 & -0.737898 & 2.881675\end{array}$ N $0.700546-1.369725 \quad 3.664236$ $\mathrm{H}-1.887784-1.374703 \quad 1.021882$ $\begin{array}{lllll}\mathrm{H} & -1.887784 & -1.374703 & 1.02188\end{array}$ $\begin{array}{llll}\mathrm{H} & -2.316993 & 0.390882 & 0.708842\end{array}$ $\begin{array}{lllll}H & -0.551026 & 1.156714 & 2.246906\end{array}$ C $-0.644559-2.896420-0.355083$ $\begin{array}{llll}\text { C } & -0.644559 & -2.896420 & -0.355083 \\ \text { C } 2.211400 & -3.820987 & -0.880642\end{array}$ $\begin{array}{llll}\text { C } & 2.211400 & -3.820987 & -0.880642 \\ \text { C } 4.233928 & -1.572369 & 0.614330\end{array}$ $\begin{array}{llll}\text { C } & 4.233928 & -1.572369 & 0.614330 \\ \text { C } 3.980362 & -2.031552 & 2.078490\end{array}$ $\begin{array}{llll}\text { C } 3.980362 & -2.031552 & 2.078490 \\ \text { C } 5.131185 & -0.316661 & 0.679978\end{array}$ $\begin{array}{llll}\text { C } 5.131185 & -0.316661 & 0.679978 \\ \text { C } 5.175989 & -2.578128 & -0.097643\end{array}$ $\begin{array}{llll}\text { C } 5.175989 & -2.578128 & -0.097643 \\ \text { C } 3.140271 & -3.484533 & -2.083347\end{array}$ $\begin{array}{llll}\text { C } 2.824082 & -4.751954 & 0.200417\end{array}$ C $1.115951-4.702133-1.527154$ $\begin{array}{llll}\text { C } & -1.237291 & -2.592088 & -1.599065\end{array}$ $\begin{array}{llll}\text { C }-1.243331 & -3.768849 & 0.573353\end{array}$ $\begin{array}{llll}\text { C }-2.421624 & -3.252610 & -1.928946\end{array}$ $\begin{array}{llll}\text { C }-2.432206 & -4.400368 & 0.180317\end{array}$ C $-3.015813-4.162825-1.056590$ $\begin{array}{llll}\text { C }-3.015813 & -4.162825 & -1.056590 \\ \text { B }-0.608610 & -1.518242 & -2.632270\end{array}$ $\begin{array}{llll}\text { B }-0.608610 & -1.518242 & -2.632270 \\ \text { F }-0.427522 & -0.225574 & -1.881798\end{array}$ F $0.685995-1.867210-3.085680$ F $-1.457111-1.867210-3.085680$ $\begin{array}{llll}\text { F }-1.457111 & -1.239426 & -3.700609\end{array}$ $\begin{array}{llll}\text { C } & -0.698500 & -4.059341 & 1.962449 \\ \text { C }-0.387621 & -5.561142 & 2.163679\end{array}$ $\begin{array}{llll}\text { C }-0.387621 & -5.561142 & 2.163679\end{array}$ C $-1.681450-3.6108353 .057259$ $\begin{array}{llll}\text { H } & 0.235138 & -3.494091 & 2.098862\end{array}$ H $0.272272-5.9614301 .380564$ H $0.095156-5.715692 \quad 3.142399$ H $-1.315895 \quad-6.1553332 .148655$ H $-1.962032-2.5534402 .957479$ H $-1.227648 \quad-3.742735 \quad 4.050923$ н $-2.605764-4.208757 \quad 3.015081$ H $-2.878137-3.032593-2.895443$ H $-3.939682-4.673488-1.332170$ $\begin{array}{lllll}\mathrm{H} & -3.939682 & -4.673488 & -1.332170 \\ \mathrm{H} & -2.910535 & -5.095301 & 0.871894\end{array}$ $\begin{array}{lllll}\text { H } & -2.910535 & -5.095301 & 0.871894\end{array}$ $\begin{array}{llll}\text { C } 3.157695 & 1.591041 & -1.316077 \\ \text { C } 3.023861 & 1.945478 & 1.111530\end{array}$ $\begin{array}{llll}\text { C } 3.742159 & 2.855347 & -1.434839\end{array}$ C 3.6071173 .2008010 .930202 $\begin{array}{llll}\text { C } 3.983071 & 3.656381 & -0.325743\end{array}$ $\begin{array}{llll}\text { C } 2.883506 & 0.785488 & -2.578099\end{array}$ $\begin{array}{llll}\text { C } 2.174369 & 1.614403 & -3.659437\end{array}$ $\begin{array}{llll}\text { C } 4.175300 & 0.180909 & -3.149092\end{array}$ H $2.205653-0.041409-2.329435$ H $1.277262 \quad 2.110556-3.263181$ H $1.8566870 .949660-4.476010$ H $2.8360232 .384773-4.086122$ H $4.681655-0.466443-2.418438$ H $3.946242-0.423999-4.039825$ $\begin{array}{llll}\text { H } 3.946242 & -0.423999 & -4.039825\end{array}$ $\begin{array}{llll}\text { H } & 4.884711 & 0.973357 & -3.439920\end{array}$ $\begin{array}{llll}\text { C } 2.621273 & 1.556135 & 2.529904\end{array}$ C 1.5972802 .5429153 .118268 $\begin{array}{llll}C & 3.798055 & 1.496336 & 3.521955\end{array}$ H $2.162845 \quad 0.5594362 .493796$ $\begin{array}{lllll}\text { H } & 0.766945 & 2.755731 & 2.43274\end{array}$ H 2.0784423 .5061683 .350817 H $1.1873192 .140974 \quad 4.057896$ H $4.5624750 .757461 \quad 3.246799$ H $4.2914302 .478994 \quad 3.602634$ H $3.416878 \quad 1.225396 \quad 4.519860$ H $4.0026813 .223058 \quad-2.429574$ H $4.442776 \quad 4.640521-0.444068$ H $3.767730 \quad 3.838080 \quad 1.802324$ H $6.109431-0.645741 \quad 1.064344$ H $5.2986110 .130960-0.309512$ 4.7681590 .4603081 .348833 $4.953333-2.2091942 .565036$ $\begin{array}{lllll}\text { H } 4.953333 & -2.209194 & 2.565036\end{array}$ . $3.480978-1.2608112 .645411$ $3.387331-2.9525292 .148379$ H $6.041649-2.7134310 .569272$ $\begin{array}{llll}\text { H } & 4.777514 & -3.565053 & -0.312555\end{array}$ H $5.557896-2.151759-1.037192$ $\begin{array}{llll}\text { H } 3.826436 & -4.325048 & -2.273439\end{array}$ н $2.511724-3.328962-2.970209$ н $3.734715-2.575303-1.962635$ H $3.225291-5.648175-0.300355$ H $3.616936 \quad-4.324698 \quad 0.815514$ H $2.026753-5.080003 \quad 0.881824$ H $1.655787-5.540359-1.999505$ H $0.405078-5.130059-0.811489$ н $0.568760-4.173592-2.314120$ $\mathrm{H}$
100

$20 \mathrm{R}=\mathrm{n}-\mathrm{Pr} \quad \mathrm{AC} / \mathrm{N}$

Ni $0.061629-0.069055 \quad 0.010343$ N $1.968570-0.080244-0.111793$ N $0.401143-1.983205-0.182789$ C $1.611688-2.400179-0.448925$ C $2.607532-1.226856-0.267302$ $\begin{array}{llll}\text { C } & -0.180192 & 1.915490 & 0.039602\end{array}$ C $-1.513935 \quad 2.527278-0.35356$ C $-1.3673114 .036708-0.597692$ $\begin{array}{lllll}\text { H } & 0.596267 & 2.230434 & -0.669143\end{array}$ H $0.117098 \quad 2.210821 \quad 1.058506$ H $-2.266096 \quad 2.376080 \quad 0.435756$ H $-1.8995802 .045508-1.267030$ H $-0.674856 \quad 4.236051-1.429140$ н $-0.971760 \quad 4.5444450 .296222$ H $-2.3400304 .489227-0.846838$ H $-2.340030-4.48927-0.846838$ $\begin{array}{llll}\mathrm{N}-1.624305 & -0.137044 & 0.642704\end{array}$ C $-2.757555-0.1763500 .911958$

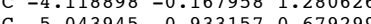
C -5.0439450 .9331570 .679299$ H -4.3870710 .5187062 .088498$ $\begin{array}{lllll}\mathrm{H} & -6.085331 & -0.882044 & 0.995759\end{array}$ C $-1.103095-5.0152902 .405399$ $\begin{array}{llll}\text { C } 2.621519 & 1.194646 & 0.008782\end{array}$ C $-0.837676-2.659366-0.458652$ $\begin{array}{llll}\text { C } 1.918307 & -3.909150 & -0.793161\end{array}$ $\begin{array}{llll}\text { C } 4.158870 & -1.365656 & -0.31735\end{array}$ $\begin{array}{llll}\text { C } 4.586590 & -1.535428 & -1.82233\end{array}$ $\begin{array}{llll}\text { C } 5.030367 & -0.183399 & 0.178801\end{array}$ $\begin{array}{llll}C & 4.638794 & -2.520139 & 0.595488\end{array}$ $\begin{array}{llll}\text { C } 2.091823 & -4.668004 & 0.551857\end{array}$

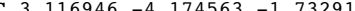
C $3.116946-4.174563-1.732910$ C $0.778239-4.644930-1.549235$ $\begin{array}{llll}C & -1.362980 & -2.424894 & -1.747452\end{array}$ C $-1.540069-3.3439430 .548308$ C $-2.600333-3.003499-2.036903$

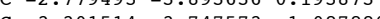
$\begin{array}{lllll}C & -3.301514 & -3.747573 & -1.087880\end{array}$ B $-0.623810-1.463854-2.844504$ F $-1.352501-1.410571-4.040359$ F $-0.566900-0.117639-2.272415$ F $0.724893-1.862042-3.105144$ C $-1.006052-3.546609 \quad 1.959117$ $\begin{array}{llll}\text { C }-1.716367 & -2.658620 & 2.994545\end{array}$ H $0.055017 \quad-3.254706 \quad 1.960193$ H $-0.704743-5.707771 \quad 1.649434$ H $-0.548855-5.165003 \quad 3.344997$ $\mathrm{H}-2.150300-5.299605 \quad 2.592772$ H $-1.515488-1.5942852 .816640$ $4-1.515488-1.5942852 .816640$ H $-1.367730-2.9093904 .009390$ H $-3.8007711-2.8189642 .959972$ $1-3.007711-2.856160-3.039678$ $\begin{array}{llll}\text { H } & -4.256293 & -4.211622 & -1.342793 \\ \text { H } & -3.339589 & -4.463974 & 0.937262\end{array}$

C $2.979348 \quad 1.908023-1.156641$ $\begin{array}{llll}\text { C } 2.732695 & 1.771141 & 1.291875\end{array}$ C $3.563338 \quad 3.166672-0.990814$ C $3.345635 \quad 3.020802 \quad 1.399424$ $\begin{array}{llll}\text { C } 3.776936 & 3.712548 & 0.271960\end{array}$ $\begin{array}{llll}\text { C } 2.745462 & 1.396604 & -2.570045\end{array}$ C $1.550003 \quad 2.093319-3.242020$ C $4.0014291 .598171-3.488776$ H $2.513657 \quad 0.323100-2.534733$ $\mathrm{H} 0.6019371 .836827-2.755489$ $\begin{array}{llll}\mathrm{H} & 0.601937 & 1.836827 & -2.755489\end{array}$ H $1.6863383 .187113-3.233404$ $\begin{array}{llll}\mathrm{H} & 1.474434 & 1.759640 & -4.288368\end{array}$ H $4.941877-1.307229-2.994368$ H $4.0940462 .654285-3.793257$

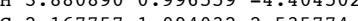
$\begin{array}{llll}\text { C } & 2.167757 & 1.094032 & 2.535774\end{array}$ C $1.567062 \quad 2.101613 \quad 3.52590$ $\begin{array}{llll}\text { C } 3.183450 & 0.208097 & 3.271842\end{array}$ H 1.3465200 .4405862 .193050 $\begin{array}{llll}\mathrm{H} & 3.503617 & -0.643321 & 2.658144\end{array}$ H $4.077167 \quad 0.787962 \quad 3.555324$ H $2.731824-0.194773 \quad 4.192466$ H $0.892915 \quad 2.812942 \quad 3.026847$ H $2.349606 \quad 2.678115 \quad 4.043574$ н $0.9950861 .564075 \quad 4.297509$ H $3.8611263 .732209-1.875698$ $\begin{array}{llll}H & 0.861126 & 3.732209 & -1.875698\end{array}$ $\begin{array}{llll}\text { H } & 4.251904 & 4.691041 & 0.374505\end{array}$ H $-4.773897-1.612843-0.128943$ $\mathrm{H}-4.773897-1.612843-0.1289$ H $6.075427-0.5245430 .074872$ H $4.9315790 .731534-0.413626$ H 4.8699590 .0620201 .236643 H $5.468572-2.193883-1.892763$ H $3.796375-1.933015-2.467886$ H $4.865434-0.552670-2.221723$ H $5.733690-2.5981090 .503829$ H $4.414113-2.2838301 .648077$ H $4.221565 \quad-3.499448 \quad 0.376298$ H $3.195875-5.266754-1.842664$ н $2.910982-3.759647-2.730061$ н $4.089011-3.821973-1.394865$ H $1.162829-5.650305-1.783027$ H $-0.136070-4.780433-0.966241$ $\begin{array}{llll}\text { H } & -0.136070 & -4.780433 & -0.966241 \\ \text { H } & 0.537191 & -4.138005 & -2.491966\end{array}$ $\begin{array}{llll}\text { H } & 0.537191 & -4.138005 & -2.491966 \\ \text { H } & 2.536980 & -5.655548 & 0.348113\end{array}$ $\begin{array}{llll}\text { H } & 2.536980 & -5.655548 & 0.348113 \\ \mathrm{H} & 2.720490 & -4.154308 & 1.289541\end{array}$ $\begin{array}{llll}\mathrm{H} & 2.720490 & -4.154308 & 1.289541 \\ \mathrm{H} & 1.112608 & -4.834640 & 1.013547\end{array}$ 100

$20 \mathrm{R}=\mathrm{CHCNE} \mathrm{ET} / \mathrm{p}$

Ni $0.067288-0.115315-0.057358$ N $2.107990 \quad-0.113834-0.053417$ C $2.755056 \quad 1.169742 \quad 0.076770$ $\begin{array}{lll}\text { C } 2.701128 & -1.279897 & -0.079930\end{array}$ $\begin{array}{llll}\text { C } 4.175797 & -1.572324 & 0.359313\end{array}$ $\begin{array}{llll}\text { C } 1.675105 & -2.422241 & -0.322755\end{array}$ C $2.026436-3.719938-1.104495$ $\begin{array}{lllll}\mathrm{N} & 0.476408 & -2.060099 & 0.028304\end{array}$ C $-0.718327-2.856935-0.054497$ C $-1.548427-2.665829-1.178786$ $\begin{array}{llll}C & -1.548427 & -2.665829 & -1.178786\end{array}$ $\begin{array}{llll}C & -2.764125 & -3.352333 & -1.189839\end{array}$ $\begin{array}{llll}C & -3.154358 & -4.181089 & -0.126515\end{array}$ C $-2.324249-4.3071730 .980830$ C $-1.095823-3.6371521 .054378$ $\mathrm{H}-3.424593-3.224365-2.048884$ H $-4.110777-4.708455-0.157818$ $\begin{array}{llll}\mathrm{H} & -2.638311 & -4.932483 & 1.818491\end{array}$ B $-1.187606-1.743646-2.472569$ F $-0.200902-0.681380-2.089255$ F $-0.559433-2.478686-3.497884$ F $-2.328938-1.092687-2.956931$ $\begin{array}{llll}\text { C }-0.256309 & -3.772226 & 2.312349\end{array}$ $\begin{array}{llll}\text { C } & 0.185818 & -5.255289 & 2.564059\end{array}$ C $-0.993848 \quad-3.246785 \quad 3.552756$ H $0.694269-5.682173 \quad 1.686125$ $\begin{array}{llll}-0.691712 & -5.886622 & 2.783426\end{array}$ $40.69842-5.385283-38342$ H $-1.880408-3.8623643 .770980$ $\mathrm{H}-1.880408-3.862364 \quad 3.770980$ H $-0.335160-3.283272 \quad 4.434742$ H $-1.337663-2.2117223 .414736$ $\begin{array}{llll}A & 0.659347-3.176141 & 2.183057\end{array}$ $\begin{array}{llll}\text { C } 3.115288 & 1.815825 & -1.126542\end{array}$ $\begin{array}{llll}\text { C } 3.726452 & 3.087584 & -1.047703\end{array}$ $\begin{array}{llll}\text { C } 3.929806 & 3.710318 & 0.175885\end{array}$ C $3.502414 \quad 3.074388 \quad 1.347929$ C $2.906472 \quad 1.813704 \quad 1.332191$ H $4.0238013 .591159-1.969489$ $\begin{array}{lllll}\text { H } & 4.393378 & 4.698221 & 0.226145\end{array}$ $\begin{array}{lllllll}\text { H } 3.628664 & 3.585847 & 2.303805\end{array}$ $\begin{array}{llll}\text { C } 2.894153 & 1.192028 & -2.497995\end{array}$ $\begin{array}{llll}\text { C } 2.357403 & 2.203102 & -3.527488\end{array}$ 
$\begin{array}{llll}\text { H } & 1.520180 & 2.792172 & -3.127162\end{array}$ C $2.473053 \quad 1.231632 \quad 2.677738$ C 3.6460600 .9224583 .630769 C $1.539476 \quad 2.198567 \quad 3.434086$ $\begin{array}{lllll}\text { H } & 1.926789 & 0.297144 & 2.481971\end{array}$ $\begin{array}{lllll}\text { H } & 4.320002 & 0.133994 & 3.276224\end{array}$ H $4.249557 \quad 1.828243 \quad 3.799390$ H $3.244637 \quad 0.605142 \quad 4.606232$ $\begin{array}{llll} & 0.796942 & 2.671481 & 2.781185\end{array}$ H 1.028648 $\begin{array}{llll}\text { H } & 1.028648 & 1.663704 & 4.249843\end{array}$ H 2.1257433 .0103413 .893976 $\begin{array}{lllll}\text { C } 4.011994 & -2.296045 & 1.724792\end{array}$ H $5.007732-2$. H $3.529542-1.6258712 .450418$ H $3.408851-3.2102871 .668922$ $\begin{array}{llll}\text { C } 5.065293 & -0.337246 & 0.619488\end{array}$ $\begin{array}{lllll}\text { H } & 5.216680 & 0.270291 & -0.282459\end{array}$ H $6.050821-0.7200550 .930381$ H 4.7062210 .3161821 .412161 C $5.068396-2.393421-0.611356$ H $4.645296-3.299829-1.033246$ H $5.397717-1.758551-1.44688$ H $5.972255-2.677726-0.049411$ C $2.638055-3.220755-2.442654$ H $1.821536-2.853539-3.083182$ H $3.128706-4.064839-2.954551$ H $3.367884-2.410958-2.341886$ C $3.367884-2.410958-2.341886$ $\begin{array}{llll}\text { C } 2.909446 & -4.722110 & -0.321603\end{array}$ $\begin{array}{llll}H & 3.215319 & -5.519744 & -1.020355\end{array}$ H $2.303172-5.1959260 .467072$ $\begin{array}{llll}\text { H } & 3.812775 & -4.335376 & 0.152543 \\ \text { C } 0.846938 & -4.616370 & -1.546921\end{array}$ $\begin{array}{lllll}\text { C } & 0.846938 & -4.616370 & -1.546921 \\ \text { H } & 0.146491 & -4.103320 & -2.214946\end{array}$ $\begin{array}{llll}\mathrm{H} & 0.146491 & -4.103320 & -2.214946 \\ \mathrm{H} & 1.299571 & -5.441375 & -2.125259\end{array}$ $\begin{array}{lllll}\mathrm{H} & 1.299571 & -5.441375 & -2.125259 \\ \mathrm{H} & 0.297374 & -5.065004 & -0.707446\end{array}$ C $-0.310577 \quad 1.735027-0.760269$ C $-1.6449561 .897167-1.510764$ H $0.530499 \quad 1.721371-1.469385$ $\begin{array}{llll}\text { C }-0.130569 & 2.794884 & 0.182912\end{array}$ C $-1.723554-0.102643 \quad 0.926370$ C $-0.654310 \quad 0.162272 \quad 1.789126$ H $-2.157316-1.101892 \quad 0.848935$ H $-2.367350 \quad 0.711264 \quad 0.594986$ $\begin{array}{llll}\mathrm{H} & -2.367350 & 0.711264 & 0.594986\end{array}$ $\begin{array}{llll}\mathrm{H}-0.461810 & 1.181810 & 2.124867\end{array}$ $\begin{array}{llll}\mathrm{H} & -0.234549 & -0.633409 & 2.410633\end{array}$ $\mathrm{N}-0.084238 \quad 3.6795710 .948710$ $\begin{array}{llll}C & -1.563767 & 3.016932 & -2.556093\end{array}$ H $-2.441996 \quad 2.144485-0.793889$ H $-1.909446 \quad 0.954542 \quad-2.006217$ $\begin{array}{llll}\mathrm{H} & -2.546090 & 3.151102 & -3.035478\end{array}$ H $-1.2686333 .973565-2.096743$ $\begin{array}{lllll}\mathrm{H} & -0.837969 & 2.765349 & -3.343882\end{array}$ 10

$20 \mathrm{R}=\mathrm{CHCNE} \mathrm{AC} / \mathrm{pi}$

Ni $0.172860 \quad-0.107706 \quad 0.052775$ N $2.235563-0.154963 \quad 0.064849$ N $0.535664-2.081992-0.004560$ $\begin{array}{llll}\text { C } 1.759338 & -2.461144 & -0.219834\end{array}$ C $2.802481-1.3324900 .088619$ $\begin{array}{llll}C & 2.802481 & -1.332490 & 0.088619\end{array}$ $\begin{array}{llll}\text { C }-0.231449 & 1.813871 & -0.50086\end{array}$ $\begin{array}{llll}C & -1.585790 & 1.985401 & -1.21176\end{array}$ $\begin{array}{llll}C & -1.586588 & 3.225789 & -2.114935\end{array}$ $\begin{array}{llll}C & -0.081119 & 2.8245110 .498685\end{array}$ $\mathrm{N}-0.0788383 .6720061 .306534$ H $0.6045461 .868115-1.214095$ H $-2.3818472 .103151-0.461176$ H $-1.8006491 .092792-1.812638$ H $-0.8616323 .115476-2.934129$ н $-1.3373704 .133493-1.544783$ H $-2.583399 \quad 3.360375-2.561237$ C $-1.546138 \quad-0.217597 \quad 1.065420$ $\begin{array}{llll}\text { C } & -0.478526 & 0.143838 & 1.925756\end{array}$ $\begin{array}{llll}\text { C } 0.165930 & -0.744088 & 2.835749\end{array}$ N $0.736263-1.411387 \quad 3.607627$ H $-1.917305-1.2433191 .018067$ H -2.2594960 .5554260 .784110$ $\begin{array}{llll}\mathrm{H} & -2.259496 & 0.555426 & 0.784110 \\ \mathrm{H} & -0.389184 & 1.190974 & 2.230767\end{array}$ $\begin{array}{lllll}\mathrm{H} & -0.389184 & 1.190974 & 2.230767\end{array}$ $\begin{array}{llll}\text { C } & 2.873863 & 0.657886 & -2.832777-0.339275\end{array}$ $\begin{array}{llll}\text { C } 2.177832 & -3.813217 & -0.852007\end{array}$ $\begin{array}{llll}\text { C } & 2.177832 & -3.813217 & -0.852007\end{array}$ $\begin{array}{llll}\text { C } & 4.271130 & -1.604132 & 0.585977 \\ \text { C } 4.077135 & -2.077727 & 2.053584\end{array}$ $\begin{array}{llll}\text { C } & 4.077135 & -2.077727 & 2.053584 \\ \text { C } 5.189564 & -0.362236 & 0.606402\end{array}$ $\begin{array}{llll}\text { C } & 5.189564 & -0.362236 & 0.606402 \\ \text { C } 5.165791 & -2.619671 & -0.174078\end{array}$ $\begin{array}{llll}\text { C } 5.165791 & -2.619671 & -0.174078 \\ \text { C } 3.081009 & -3.493621 & -2.077182\end{array}$ $\begin{array}{llll}\text { C } 3.081009 & -3.493621 & -2.07718 \\ \text { C } 2.807765 & -4.739178 & 0.218718\end{array}$ $\begin{array}{llll}\text { C } 1.068924 & -4.710506 & -1.449714\end{array}$ $\begin{array}{llll}\text { C }-1.234538 & -2.547405 & -1.594576\end{array}$ $\begin{array}{llll}\text { C }-1.277281 & -3.680649 & 0.602807\end{array}$ $\begin{array}{llll}\text { C }-2.432177 & -3.190485 & -1.919627\end{array}$ $\begin{array}{llll}C & -2.471572 & -4.300132 & 0.217295\end{array}$ C $-3.052007-4.073069-1.039560$ B $-0.574394-1.563951-2.039560$ B $-0.574394-1.563951-2.695697$ F $-0.031314-0.347316-1.982022$ F $0.540130-2.123658-3.349649$ $\begin{array}{llll}\text { F } & -1.512698 & -1.088355 & -3.610536 \\ \text { C }-0.743820 & -3.960629 & 1.995722\end{array}$ $\begin{array}{llll}\text { C }-0.445926 & -5.483822 & 2.222342\end{array}$ C $-1.726769-3.493741 \quad 3.08115$ $\begin{array}{llll}\text { H } 0.198705 & -3.413623 & 2.133248\end{array}$ H $0.219458-5.8982351 .449628$ н $0.026744 \quad-5.623880 \quad 3.209056$ н $-1.381748 \quad-6.067452 \quad 2.206259$ H $-1.998886-2.4350812 .969156$ $\mathrm{H}-1.277674-3.618114 \quad 4.077587$ H $-2.655929-4.0840873 .042450$ H $-2.877169-2.980769-2.893619$ $\begin{array}{lllll}\mathrm{H} & -2.877169 & -2.980769 & -2.893619\end{array}$ $\begin{array}{lllll}\mathrm{H} & -3.985920 & -4.572269 & -1.310483\end{array}$ $\begin{array}{llll}\text { H } & -2.966273 & -4.975453 & 0.91681\end{array}$ C $3.1472901 .723213-1.157260$ $\begin{array}{llll}\text { C } 3.081642 & 1.871068 & 1.302286\end{array}$ C $3.6765963 .015946-1.188537$ C 3.5964993 .1648371 .199842 $\begin{array}{llll}\text { C } 3.902848 & 3.739330 & -0.023389\end{array}$ $\begin{array}{llll}\text { C } 2.897816 & 0.988883 & -2.469102\end{array}$ $\begin{array}{llll}\text { C } 2.316203 & 1.898544 & -3.562819\end{array}$ $\begin{array}{llll}\text { C } 4.170080 & 0.309942 & -2.999274\end{array}$ H $2.154178 \quad 0.201709 \quad-2.286394$ H $1.468895 \quad 2.493309-3.192653$ H $1.958381 \quad 1.279802 \quad-4.399009$ H $3.0716662 .594829-3.960404$ H $4.561736-0.435147-2.293461$ н $3.952447-0.206133-3.947246$ $\begin{array}{lllll}\text { H } 3.952447 & -0.206133 & -3.947246 \\ \text { H } & 4.963945 & 1.053358 & -3.179666\end{array}$ $\begin{array}{llll}\text { H } & 4.963945 & 1.053358 & -3.179666\end{array}$ $\begin{array}{llll}C & 2.830041 & 1.336001 & 2.710326\end{array}$ C 1.8499252 .2056493 .513895 C 4.1085271 .2404433 .569502 H $2.401960 \quad 0.3294202 .623505$ H 0.9590862 .4930912 .947795 H $2.334324 \quad 3.145418 \quad 3.823866$ H $1.550307 \quad 1.669296 \quad 4.427353$ H $4.879917 \quad 0.577022 \quad 3.157090$ H 4.5582362 .2387713 .700830 H $3.839466 \quad 0.860266 \quad 4.568768$ H $3.901435 \quad 3.471048 \quad-2.155023$ H $4.303555 \quad 4.754375 \quad-0.073923$ H $3.7568393 .737097 \quad 2.115362$ H $6.173568-0.699923 \quad 0.967663$ H $5.3350210 .060465-0.396890$ H 4.8611400 .4375881 .263810 $6.062409-2.7583370 .450306$ H $6.062409-2.7583370 .450306$ H $4.752397-3.605023-0.364307$ H $5.505889-2.198566-1.131692$ $\begin{array}{lll}H & 5.066447-2.300721 & 2.484900\end{array}$ H $3.600440-1.2994782 .661471$ H $3.453627-2.976592 \quad 2.140773$ H $3.777803-4.328111-2.260918$ H $2.433999-3.367128-2.956795$ н $3.662358-2.571210-1.98881$ н $3.186505-5.644361-0.287080$ H $3.622143-4.3204750 .813396$ H $2.024441-5.056352 \quad 0.924225$ H $1.599763-5.565323-1.906324$ H $0.372500-5.116311-0.704385$ н $0.505269-4.211364-2.246690$ H

$20 \mathrm{R}=\mathrm{CHCNEt} \quad \mathrm{AC} / \mathrm{N}$

$\mathrm{Ni}-0.117579-0.289967 \quad 0.693383$ $\mathrm{N} 0.998843-0.226531-0.883224$ $\mathrm{N}-1.464045-0.134604-0.669777$ C $-1.098348-0.015264-1.925498$ C $0.450573-0.062215-2.059362$ C $2.398483-0.314538-0.567048$ C $-2.797409 \quad 0.142854-0.185778$ $\begin{array}{llll}\text { C }-2.098549 & -0.058610 & -3.126698\end{array}$ $\begin{array}{llll}\text { C } 1.252509 & 0.244831 & -3.353260\end{array}$ $\begin{array}{llll}\text { C } 1.045429 & 1.806277 & -3.610435\end{array}$ $\begin{array}{llll}\text { C } 0.882934 & -0.621112 & -4.585176\end{array}$ $\begin{array}{llll}\text { C } 2.785932 & 0.052784 & -3.292754\end{array}$ C $-1.843816 \quad 0.961283-4.269912$ C $-2.052670-1.542260-3.605890$ $\begin{array}{llll}\text { C }-3.589284 & 0.227112 & -2.821174\end{array}$ C $-3.054870 \quad 1.504463 \quad 0.058037$ C $-3.681854-0.8960560 .159038$ $\begin{array}{llll}C & -3.081854 & -0.896056 & 0.15903\end{array}$ C -4.2877371 .8092180 .645608$ C $-4.898007-0.5205590 .740512$ \begin{tabular}{l}
$C-5.2041030 .8153390 .983645$ \\
\hline
\end{tabular} B $-1.9886892 .691740-0.318160$ F -2.4484803 .9332830 .149165$ $\begin{array}{llll}-0.701064 & 2.405330 & 0.274493\end{array}$ F $-1.7875352 .744655-1.739846$ $\begin{array}{llll}\text { C }-3.402327 & -2.368094 & -0.116228\end{array}$ C $-4.490356-3.003686-1.000968$ $\begin{array}{llll}\text { C }-3.271118 & -3.194738 & 1.171777\end{array}$ H $-2.441808-2.431489-0.652732$ H $-4.666199-2.444069-1.930289$ H $-4.205137-4.033860-1.266281$ H $-5.448107-3.054521-0.459797$ H $-2.430724-2.8727711 .798296$ H $-3.436561-2.87565460 .921537$ $\begin{array}{llll}\mathrm{H} & -3.116561 & -4.256546 & 0.921537\end{array}$ 1.4879652 .8600670 .836380 H -4.5138652 .8600670 .836380$ H $-5.620383-1.293544 \quad 1.007748$

$\begin{array}{llll}\text { C } 3.117272 & 0.850544 & -0.233239\end{array}$ $\begin{array}{llll}\text { C } 2.949085 & -1.602914 & -0.401207\end{array}$ $\begin{array}{llll}\text { C } 4.456451 & 0.699883 & 0.137640\end{array}$ $\begin{array}{llll}\text { C } 4.294541 & -1.698948 & -0.043735\end{array}$ $\begin{array}{llll}\text { C } 5.060817 & -0.553794 & 0.203370\end{array}$ $\begin{array}{llll}\text { C } 2.496098 & 2.236690 & -0.231362\end{array}$ C $2.285575 \quad 2.733862 \quad 1.207235$ C $3.3395493 .273689-1.005167$ H $1.500752 \quad 2.185452-0.693816$ H 1.6487772 .0473391 .780374 1.6487772 .0473391 .780374 $\begin{array}{lllll}\mathrm{H} & 3.251818 & 2.836743 & 1.728264\end{array}$ 1.7851933 .7132901 .190602 H $3.6113092 .929549-2.015648$ H $4.2748303 .508530-0.469792$ H $2.7673614 .210713-1.101147$ $\begin{array}{llll}\text { C } 2.093347 & -2.854573 & -0.544448\end{array}$ $\begin{array}{llll}\text { C } 2.524162 & -3.978360 & 0.404686\end{array}$ C $2.038192-3.382252-1.984180$ H $1.066818-2.566439-0.257376$ H $5.041146 \quad 1.590417 \quad 0.373230$ H $6.117609-0.644658 \quad 0.468553$ H $4.752474 \quad-2.682225 \quad 0.065488$ н $3.1632750 .311457-4.295788$ H $3.291773 \quad 0.709558 \quad-2.582216$ н $3.083882-0.984060-3.085964$ H $1.1297912 .012614-4.691116$ $\begin{array}{llll}4 & 1.129791 & 2.012614 & -4.691116\end{array}$ H $0.0892252 .198854-3.238154$ H $1.8444782 .352887-3.089989$ $\begin{array}{llll}\mathrm{H} & 1.423416 & -0.205913 & -5.452255\end{array}$ $\begin{array}{llll}\text { H } & 1.248607 & -1.652002 & -4.442132 \\ \text { H }-0.170312 & -0.676269 & -4.852666\end{array}$ H $-0.170312-0.676269-4.852666$ $\begin{array}{llll}\text { H } & -2.559108 & 0.721936 & -5.074764\end{array}$ H $-2.070893 \quad 1.972260-3.898405$ H $-0.8529100 .976690 \quad-4.716248$ H $-2.515269-1.614013-4.603785$ н $-1.046725-1.975523-3.662139$ н $-2.636597-2.166369-2.915280$ H $-4.1182780 .127910 \quad-3.782772$ H $-4.052302-0.473943-2.126670$ H $-3.738152 \quad 1.250099-2.453969$ $\begin{array}{llll}\text { C }-1.292402 & -0.285285 & 2.351892\end{array}$ c $-1.109783 \quad 1.040228 \quad 3.110707$ C $-2.1243691 .186100 \quad 4.251099$ C $-1.028501-1.422147 \quad 3.192755$ $\begin{array}{llll}\text { C } & -1.028501 & -1.422147 & 3.192755 \\ \mathrm{~N} & -0.767743 & -2.321639 & 3.896325\end{array}$ $\begin{array}{llll}\mathrm{N} & -0.767743 & -2.321639 & 3.896325 \\ \mathrm{H} & -2.315459 & -0.364587 & 1.957368\end{array}$ H $-0.087947 \quad 1.095686 \quad 3.519119$ H $-1.224583 \quad 1.865776 \quad 2.394802$ H $-1.971554 \quad 2.142708 \quad 4.773463$ H $-3.153513 \quad 1.170765 \quad 3.860544$ H $-2.020029 \quad 0.369930 \quad 4.983116$ $\begin{array}{llll}\mathrm{N} & 1.183546 & -0.642117 & 1.892620\end{array}$ $\begin{array}{llll}\text { C } 1.901780 & -0.976854 & 2.746283\end{array}$ C $2.621266-1.454952 \quad 3.857322$ C $3.946213-1.3060974 .002670$ H $1.996370-1.961878 \quad 4.601116$ H $4.449666-1.696670 \quad 4.888658$

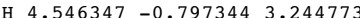
H $1.423468-4.295096-2.029674$ $\begin{array}{llll}\mathrm{H} & 1.423468 & -4.295096 & -2.029674 \\ \mathrm{H} & 1.597190 & -2.646331 & -2.668125\end{array}$ $\begin{array}{llll}\text { H } 1.597190 & -2.646331 & -2.668125\end{array}$ $\begin{array}{llll}\text { H } 3.048693 & -3.628782 & -2.349039\end{array}$ $\begin{array}{lll}1.768633 & -4.778089 & 0.395151\end{array}$ $\begin{array}{llll}\text { H } & 3.480480 & -4.428124 & 0.095046 \\ \text { H } & 2.626657 & -3.619673 & 1.439368\end{array}$

87

$21 \mathrm{R}=\mathrm{Me}$

Ni $-0.044426-0.022756-0.024476$ $\begin{array}{lllll}\mathrm{N} & 1.832361 & 0.005934 & -0.007634\end{array}$ $\begin{array}{llll}\text { C } 2.511325 & 1.278537 & 0.010519\end{array}$ $\begin{array}{llll}\text { C } 2.435782 & -1.172072 & 0.028667\end{array}$ $\begin{array}{llll}\text { C } 3.887324 & -1.396816 & 0.558438\end{array}$ $\begin{array}{lll}\text { C } 4.014258 & -0.657078 & 1.911041\end{array}$ $\begin{array}{llll}\text { C } 5.013177 & -0.882149 & -0.360365\end{array}$ $\begin{array}{llll}C & 4.178364 & -2.871420 & 0.888388\end{array}$ C $1.445514-2.296510-0.17927$ C $1.675082-3.296510-0.179271$ $\begin{array}{llll}C & 1.675082 & -3.486861 & -1.163093\end{array}$ c $2.797053-3.110677-2.155176$ C $1.950741-4.872802-0.540639$ C $0.408829-3.650999-2.040256$ $\begin{array}{llll}\mathrm{N} & 0.266747 & -1.955123 & 0.305385\end{array}$ $\begin{array}{llll}C & -0.777508 & -2.883490 & 0.552360\end{array}$ $\begin{array}{llll}\text { C }-2.060871 & -2.692319 & 0.003115\end{array}$ C $-0.588163-3.9163551 .508819$ $\begin{array}{llll}\text { C }-3.079798 & -3.611938 & 0.231616\end{array}$ $\begin{array}{llll}\text { C } & -1.626829 & -4.829407 & 1.702792\end{array}$ $\begin{array}{llll}\text { C }-2.846710 & -4.714786 & 1.042348\end{array}$ $\begin{array}{llll}\mathrm{S}-2.482348 & -1.183055 & -0.895264\end{array}$ o $-1.757697-1.180676-2.170989$ $0-3.938517-1.122370-0.919599$ o $-1.919770-0.1331770 .099161$ 
$\begin{array}{llll}\text { H } & 0.095197 & -5.536712 & 3.825533\end{array}$ H $0.317694-1.8439203 .031771$ H $-0.452104 \quad-3.031962 \quad 4.107542$ H $1.319680-2.799871 \quad 4.167583$ H $-4.054340-3.425981-0.218875$ н $-3.630445-5.454533 \quad 1.205375$ H $-1.486548 \quad-5.645005 \quad 2.410340$ C $3.1215041 .730309-1.183681$ $\begin{array}{llll}\text { C } 2.388471 & 2.122690 & 1.134615\end{array}$ C $3.6369503 .029770-1.203690$ $\begin{array}{llll}\text { C } 3.636950 & 3.029770 & -1.203690\end{array}$ $\begin{array}{llll}\text { C } 2.936645 & 3.405326 & 1.062679\end{array}$ $\begin{array}{llll}\text { C } 3.557581 & 3.865793 & -0.091800 \\ \text { C } 3.181529 & 0.895430 & -2.454400\end{array}$ $\begin{array}{llll}\text { C } & 3.181529 & 0.895430 & -2.454400 \\ \text { C } 4.493448 & 1.121194 & -3.275218\end{array}$ $\begin{array}{llll}\text { C } & 4.493448 & 1.121194 & -3.275218 \\ \text { C } & 1.965460 & 1.138913 & -3.360418\end{array}$ $\begin{array}{llll}\text { C } & 1.965460 & 1.138913 & -3.360418 \\ \text { H } & 3.167420 & -0.163292 & -2.168537\end{array}$ $\begin{array}{llll}\text { H } & 3.167420 & -0.163292 & -2.168537 \\ \text { H } & 4.485791 & 2.103708 & -3.775080\end{array}$ $\begin{array}{llll}\mathrm{H} & 4.485791 & 2.103708 & -3.775080 \\ \mathrm{H} & 4.560430 & 0.354019 & -4.064473\end{array}$ $\begin{array}{lllll}\text { H } & 4.560430 & 0.354019 & -4.064473 \\ \text { H } & 5.394564 & 1.057397 & -2.644395\end{array}$ H $1.0318410 .827882 \quad-2.876704$ H $2.0756930 .567403-4.295591$ H $1.880638 \quad 2.206966 \quad-3.617082$ C $1.665795 \quad 1.712106 \quad 2.410891$ C $0.328838 \quad 2.457702 \quad 2.555088$ C 2.5131191 .9442083 .672577 $\begin{array}{lllll}\text { H } & 1.439535 & 0.637304 & 2.341778\end{array}$ $\begin{array}{llll}\mathrm{H} & -0.337233 & 2.258351 & 1.706797\end{array}$ $\begin{array}{lllll}\mathrm{H} & -0.337233 & 2.258351 & 1.70679\end{array}$ $\begin{array}{llll}\mathrm{H} & 0.496731 & 3.544968 & 2.614054 \\ \mathrm{H} & -0.181102 & 2.138033 & 3.477060\end{array}$ $\begin{array}{llll}\text { H } & -0.181102 & 2.138033 & 3.477060 \\ \text { H } & 3.505530 & 1.477136 & 3.609032\end{array}$ $\begin{array}{llll}\text { H } & 3.505530 & 1.477136 & 3.609032 \\ \mathrm{H} & 2.658135 & 3.019664 & 3.858150\end{array}$ $\begin{array}{lllll}\text { H } & 2.658135 & 3.019664 & 3.858150 \\ \text { H } & 1.991082 & 1.530382 & 4.549011\end{array}$ $\begin{array}{llll}\mathrm{H} & 1.991082 & 1.530382 & 4.549011 \\ \mathrm{H} & 4.112320 & 3.398802 & -2.114523\end{array}$ $\begin{array}{lllll}\mathrm{H} & 4.112320 & 3.398802 & -2.114523 \\ \mathrm{H} & 3.972278 & 4.875944 & -0.132649\end{array}$ $\begin{array}{lllll}\text { H } & 3.972278 & 4.875944 & -0.132649 \\ \text { H } & 2.862521 & 4.062224 & 1.930850\end{array}$ C $-0.400010 \quad 1.681780-0.873556$ H $-1.1815362 .181411-0.283841$ H $-0.809757 \quad 1.413893-1.859373$ H $0.509542 \quad 2.291792-0.954199$ H $4.978271-0.933903 \quad 2.366364$ H $4.0078490 .429533 \quad 1.782839$ H $3.211410 \quad-0.948338 \quad 2.605093$ $\begin{array}{llll}\text { H } 3.211410 & -0.948338 & 2.605093\end{array}$ $\begin{array}{llll}\text { H } & 5.977711 & -1.140661 & 0.106679\end{array}$ $\begin{array}{llll}\text { H } & 4.990685 & -1.341497 & -1.358297 \\ \text { H } & 4.977167 & 0.208995 & -0.469849\end{array}$ $\begin{array}{llll}\text { H } & 4.977167 & 0.208995 & -0.469849 \\ \text { H } 5.138700 & -2.911262 & 1.425067\end{array}$ $\begin{array}{llll}H & .138700 & -2.911262 & 1.425067\end{array}$ $\begin{array}{llll}\text { H } 3.415357 & -3.298596 & 1.554987 \\ \text { H } & 4.278794 & -3.508743 & 0.004998\end{array}$ $\begin{array}{llll}\text { H } & 4.278794 & -3.508743 & 0.004998 \\ \text { H } 2.897132 & -3.924129 & -2.890502\end{array}$ $\begin{array}{llll}\text { H } & 2.897132 & -3.924129 & -2.890502 \\ \text { H } & 2.530167 & -2.196548 & -2.708208\end{array}$ $\begin{array}{llll}\text { H } & 2.530167 & -2.196548 & -2.708208 \\ \text { H } & 3.777850 & -2.966928 & -1.692128\end{array}$ $\begin{array}{lllll}\text { H } & 3.777850 & -2.966928 & -1.692128 \\ \text { H } & 0.677054 & -4.288100 & -2.897502\end{array}$ $-0.408959-4.151693-1.506119$ н $0.035336-2.689456-2.419196$ H $2.096984-5.587454-1.367001$ н $2.839843-4.9143830 .098542$ $\mathrm{H}$

$21 \mathrm{R}=\mathrm{Me} \mathrm{Et/pi}$

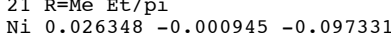
N $2.035154-0.011530-0.018148$ $\begin{array}{llll}\mathrm{N} & 2.035154 & -0.011530 & -0.018148 \\ \mathrm{C} & 2.700006 & 1.264543 & 0.039555\end{array}$ $\begin{array}{llll}\text { C } 2.653437 & -1.174560 & -0.051308\end{array}$ $\begin{array}{llll}\text { C } & 2.653437 & -1.174560 & -0.051308 \\ \text { C } & 4.082367 & -1.440198 & 0.556042\end{array}$ $\begin{array}{llll}\text { C } & 4.082367 & -1.440198 & 0.556042 \\ \text { C } 1.691026 & -2.299554 & -0.473853\end{array}$ $\begin{array}{llll}\text { C } & 1.691026 & -2.299554 & -0.473853 \\ \text { C } 2.172504 & -3.588028 & -1.193739\end{array}$ $\begin{array}{llll}\text { C } & 2.172504 & -3.588028 & -1.193739 \\ \mathrm{~N} & 0.443619 & -1.949618 & -0.315705\end{array}$ C $-0.712474-2.728544-0.627833$ C $-1.413437-2.400989-1.805938$ $\begin{array}{llll}\text { C }-2.604936 & -3.039040 & -2.121504\end{array}$ C $-3.128603-3.994195-1.252685$ C $-2.462597-4.280005-0.068604$ C $-1.256466-3.657971 \quad 0.280218$ H $-3.109461-2.761089-3.047372$ H $-4.063716-4.500985-1.491874$ $\mathrm{H}-2.892557-5.0101960 .618040$ S $-0.782887-1.121940-2.931384$ $\begin{array}{lllll}\mathrm{S} & -0.782887 & -1.121940 & -2.931384 \\ \mathrm{O} & -0.677614 & 0.087307 & -2.010214\end{array}$ o $0.536131-1.589844-3.389354$ $0-1.816934-0.930786-3.946597$ C $-0.619253-4.007072 \quad 1.616420$ C $-0.343843-5.514241 \quad 1.749947$ C $-1.499693 \quad-3.553341 \quad 2.793965$ H $0.223248 \quad-5.913460 \quad 0.897875$ H $-1.286967 \quad-6.079245 \quad 1.811647$ H $0.223288 \quad-5.7162402 .672023$ H $-2.449471-4.1112832 .801167$ H $-0.987880-3.749611 \quad 3.749451$ H $-1.744319-2.484286 \quad 2.740545$ H $0.342217 \quad-3.474810 \quad 1.684778$ C $3.1384861 .785009-1.198183$ C $3.7653583 .040828-1.206397$ C $3.9243243 .783473-0.044567$ $\begin{array}{lllll}\text { C } 3.411215 & 3.276671 & 1.159737\end{array}$ C $2.784198 \quad 2.032008 \quad 1.225588$ $\begin{array}{llll}\text { H } & 4.123237 & 3.442395 & -2.156349 \\ \text { H } & 4.417236 & 4.758167 & -0.066491\end{array}$
H $3.505362 \quad 3.873314 \quad 2.069852$ $\begin{array}{llll}\text { C } 2.935028 & 1.066427 & -2.52385\end{array}$ C $2.0747891 .905147-3.481706$ C $4.2723720 .695454-3.181476$ $\begin{array}{lllll}\text { H } 2.376331 & 0.138434 & -2.347870\end{array}$ H $4.8532491 .594831-3.443237$ H $4.0900180 .128067-4.106907$ H $4.8972410 .078868-2.517274$ H $2.5743082 .848934-3.753235$ $\mathrm{H} 1.888503 \quad 1.333801-4.403466$ $\begin{array}{llll}\mathrm{H} & 1.888503 & 1.333801 & -4.403466 \\ \mathrm{C} & 2.238398 & 2.138269 & -3.031021\end{array}$ $\begin{array}{llll}\text { C } & 2.238398 & 1.585790 & 2.578031\end{array}$ $\begin{array}{llll}\text { C } & 2.238398 & 1.585790 & 2.578031 \\ \text { C } 3.320760 & 1.404050 & 3.659656\end{array}$ $\begin{array}{llll}\text { C } & 3.320760 & 1.404050 & 3.659656 \\ \text { C } & 1.211724 & 2.600078 & 3.114276\end{array}$ H $1.730751 \quad 0.621851 \quad 2.431301$ H $4.0425850 .611053 \quad 3.425972$ H $3.8847282 .338805 \quad 3.806808$ H $2.841908 \quad 1.148897 \quad 4.618490$ $\begin{array}{llll}\text { H } & 0.466133 & 2.870231 & 2.356969\end{array}$ $\begin{array}{llll}\text { H } & 0.697145 & 2.191124 & 3.998038\end{array}$ H $1.714422 \quad 3.528735 \quad 3.427717$ C $3.762985-2.067978 \quad 1.941934$ н $4.707481-2.2981292 .461779$ H $3.195135-1.3555492 .558681$ H $3.175023-2.992712 \quad 1.875068$ $\begin{array}{llll}\text { C } 4.940585 & -0.186210 & 0.831801\end{array}$ $\begin{array}{llll}C & 4.940585 & -0.186210 & 0.831801\end{array}$ H $5.1783690 .369469-0.085650$ $\begin{array}{llll}\mathrm{H} & 5.890566 & -0.540574 & 1.262859\end{array}$ $\begin{array}{llll}\text { H } & 4.501835 & 0.507281 & 1.546364 \\ \text { C } 5.087745 & -2.327677-0.222414\end{array}$ $\begin{array}{llll}\text { C } 5.087745 & -2.327677 & -0.222414 \\ \text { H } 4.732826 & -3.293049 & -0.571250\end{array}$

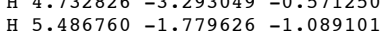
$\begin{array}{lllll}\text { H } & 5.486760 & -1.779626 & -1.089101 \\ \text { H } & 5.933587 & -2.514727 & 0.457812\end{array}$ $\begin{array}{llll}\text { H } & 5.933587 & -2.514727 & 0.457812 \\ \text { C } & 3.103308 & -3.132967 & -2.358060\end{array}$ $\begin{array}{llll}\text { C } 3.103308 & -3.132967 & -2.358060 \\ \text { H } 2.472832 & -2.866520 & -3.216650\end{array}$ $\begin{array}{llll}\text { H } & 2.472832 & -2.866520 & -3.216650 \\ \text { H } & 3.771996 & -3.961475 & -2.642257\end{array}$ H $3.716519-2.255424-2.136701$ C $2.803252-4.606104-0.208214$ H $3.214073-5.443611-0.795067$ H $2.017377-5.015500 \quad 0.442038$ H $3.597194-4.230105 \quad 0.438011$ $\begin{array}{llll}\text { C } 1.087660 & -4.416345 & -1.920341\end{array}$ H $0.542696-3.819016-2.659840$ $\begin{array}{llll}\mathrm{H} & 1.631450 & -5.203139 & -2.469535\end{array}$ H $0.381886-4.917181-1.247140$ C $-0.3061091 .932912-0.152853$ H $-1.0729772 .043670-0.929824$ H $0.643524 \quad 2.395469-0.452391$ $\mathrm{H}-0.6565332 .3315510 .806760$ $\begin{array}{llll}\text { C }-1.748199 & -0.153077 & 0.847946\end{array}$ C $-0.695493 \quad-0.104037 \quad 1.775419$ H $-2.198974 \quad-1.103844 \quad 0.550244$ H $-2.3673190 .728503 \quad 0.670991$ H $-0.509753 \quad 0.810114 \quad 2.339211$ $\begin{array}{llll}\mathrm{H} & -0.304601 & -1.019202 & 2.227100\end{array}$ 94

$21 \mathrm{R}=\mathrm{Me} \mathrm{AC} / \mathrm{pi}$

Ni $0.000046-0.0004320 .003202$ $\begin{array}{llll}\mathrm{N} & 2.038017 & -0.001147 & 0.003359\end{array}$

$\begin{array}{llll}\mathrm{N} & 2.038017 & -0.001147 & 0.003359\end{array}$

$\begin{array}{lllll}\text { C } 2.639310 & -1.166881 & 0.000782\end{array}$

$\begin{array}{llll}\text { C } & 2.639310 & -1.166881 & 0.000782 \\ \text { C } & 4.073593 & -1.431029 & 0.596664\end{array}$ C $1.660665-2.301560-0.418204$ C $2.160610-3.573121-1.151281$ $\mathrm{N} 0.414186-1.973921-0.236323$ $\begin{array}{llll}\text { C }-0.732915 & -2.754851 & -0.612668\end{array}$ C $-1.389221-2.386836-1.80570$ $\begin{array}{llll}\text { C }-2.556095 & -3.021247 & -2.20796\end{array}$ C $-3.104510-4.022530-1.410958$ $\begin{array}{llll}\text { C }-2.483496 & -4.356647 & -0.216810\end{array}$ $\begin{array}{llll}\text { C }-1.302092 & -3.739562 & 0.219465\end{array}$ H $-3.019053-2.706963-3.143567$ H $-4.020394-4.529302-1.715523$ H $-2.927726-5.127957 \quad 0.412783$ S $-0.726450-1.072942-2.858723$ $\begin{array}{llll}\mathrm{S} & -0.726450 & -1.072942 & -2.858723 \\ \mathrm{O} & -0.672239 & 0.107072 & -1.887781\end{array}$ o $0.613216-1.511015-3.280904$ $\begin{array}{llll}0 & 0.613216 & -1.511015 & -3.280904 \\ 0 & -1.718126 & -0.835152 & -3.904375\end{array}$ $\begin{array}{llll}\text { C }-0.728543 & -4.174328 & 1.558161\end{array}$ C $-0.416763-5.680662 \quad 1.591232$ $\begin{array}{llll}\text { C }-1.695811 & -3.843542 & 2.707749\end{array}$ H $0.215055-6.0006820 .751277$ H $-1.343048-6.275035 \quad 1.550244$ H $0.098858-5.9346552 .530350$ H $-2.613078-4.448440 \quad 2.626946$ H $-1.219161-4.062722 \quad 3.674713$ H $-1.987854-2.7848812 .712460$ H $0.203788-3.620480 \quad 1.737300$ C $3.1174361 .747743-1.265932$ C $3.744246 \quad 3.012153-1.341134$ C $3.926568 \quad 3.799908-0.215295$ $\begin{array}{llll}\text { C } 3.445803 & 3.336423 & 1.022707\end{array}$ $\begin{array}{llll}\text { C } 2.823280 & 2.094976 & 1.153817\end{array}$ H $4.080578 \quad 3.371720-2.315957$ H $4.416418 \quad 4.773824-0.285862$ H $3.5634523 .967093 \quad 1.907155$ C $2.8995240 .981480-2.560419$ $\begin{array}{llll}\text { C } 2.019797 & 1.780011 & -3.534767\end{array}$ C $4.230057 \quad 0.597848-3.225045$ H $2.349948 \quad 0.056879 \quad-2.346556$ H $4.796172 \quad 1.491558-3.534095$ H $4.037881-0.009015-4.123036$ H $4.8739020 .016498 \quad-2.547213$ H $2.5130362 .711760 \quad-3.854912$ H $1.815507 \quad 1.170577 \quad-4.427697$ $\begin{array}{lllll}\text { H } & 1.054404 & 2.032491 & -3.074219\end{array}$ C $2.315568 \quad 1.7076332 .537388$ $\begin{array}{llll}\text { C } 2.315568 & 1.707633 & 2.537388\end{array}$ $\begin{array}{llll}\text { C } 3.426715 & 1.578253 & 3.612391\end{array}$ $\begin{array}{llll}\text { H } & 1.825749 & 0.729162 & 2.456622\end{array}$ H $1.8257490 .729162 \quad 2.456622$ $\begin{array}{lllll}H & 4.138785 & 0.769174 & 3.403655\end{array}$ H 3.9940002 .5189413 .702012 H $2.959788 \quad 1.366664 \quad 4.587642$ $\begin{array}{lllll}\text { H } & 0.540298 & 2.989470 & 2.299652\end{array}$ H $0.793604 \quad 2.355027 \quad 3.958747$ H $1.800286 \quad 3.674978 \quad 3.334552$ $\begin{array}{llll}\text { C } 3.761690 & -2.030187 & 1.997379\end{array}$ H $4.713753-2.2645782 .501136$ H $3.205243-1.311249 \quad 2.614321$ H $3.157103-2.945171 \quad 1.959405$ $\begin{array}{llll}\text { C } 4.931702 & -0.171369 & 0.838358\end{array}$ H $5.1686790 .362753-0.092257$ H $5.881689-0.515044 \quad 1.277383$ $\begin{array}{llll}\text { H } & 5.881689 & -0.515044 & 1.277383\end{array}$ $\begin{array}{llll}\text { H } & 4.493219 & 0.535804 & 1.538562 \\ \text { C } 5.075074 & -2.338041 & -0.164844\end{array}$ $\begin{array}{llll}\text { C } & 5.075074 & -2.338041 & -0.164844 \\ \text { H } & 4.718960 & -3.312300 & -0.486929\end{array}$ H $5.476538-1.813347-1.044592$ $\begin{array}{llll}\text { H } & 5.476538 & -1.813347 & -1.044592 \\ \text { H } 5.919092 & -2.509292 & 0.521337\end{array}$ $\begin{array}{llll}\text { H } 5.919092 & -2.509292 & 0.521337 \\ \text { C } 3.115099 & -3.105180 & -2.290855\end{array}$ $\begin{array}{llll}\text { C } 3.115099 & -3.105180 & -2.290855 \\ \text { H } 2.507082 & -2.869325 & -3.173722\end{array}$ $\begin{array}{llll}\text { H } & 2.507082 & -2.869325 & -3.173722 \\ \text { H } & 3.818315 & -3.915204 & -2.541508\end{array}$ $\begin{array}{llll}\text { H } 3.818315 & -3.915204 & -2.541508 \\ \text { H } 3.694002 & -2.206162 & -2.063408\end{array}$ $\begin{array}{llll}\text { H } 3.694002 & -2.206162 & -2.063408 \\ \text { C } 2.772808 & -4.586825 & -0.148617\end{array}$ $\begin{array}{llll}\text { C } 2.772808 & -4.586825 & -0.148617 \\ \text { H } 3.216121 & -5.415797 & -0.723840\end{array}$ $\begin{array}{lllll}\text { H } & 1.971034 & -5.006384 & 0.474197\end{array}$ $\begin{array}{lllll}\text { H } 3.535198 & -4.198013 & 0.527014\end{array}$ C $1.101480-4.406800-1.909434$ H $0.564492-3.809970-2.655016$ н $1.669070-5.179899-2.454033$ H $0.388372-4.924787-1.258573$

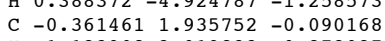
$\begin{array}{lllll}\mathrm{H} & -1.123908 & 2.019833 & -0.873087\end{array}$ H $0.5904862 .390505-0.394664$ H $-0.719023 \quad 2.353343 \quad 0.858239$ C $-1.688414-0.214673 \quad 1.011343$ C $-0.6198210 .022331 \quad 1.918420$ H $-2.078724 \quad-1.222505 \quad 0.847345$ H $-2.387383 \quad 0.600447 \quad 0.816563$ H $-0.530797 \quad 1.0193912 .354941$ C $0.030721-0.961603 \quad 2.714530$ N $0.625756-1.697436 \quad 3.402275$ 94

$21 \mathrm{R}=\mathrm{Me} \quad \mathrm{AC} / \mathrm{N}-\mathrm{t}$

Ni $0.014637-0.019028-0.058443$ N $1.907534-0.005144-0.006659$ N $0.360143 \quad 1.895122 \quad-0.048007$

$\begin{array}{llll}\text { C } 1.579923 & 2.318648 & 0.203535\end{array}$ $\begin{array}{llll}\text { C } 2.561282 & 1.155496 & 0.078217\end{array}$ C $2.566297-1.276181-0.065018$ S $-1.134611 \quad 1.4005542 .508521$ $\begin{array}{lllll}0 & -0.989437 & 0.056206 & 1.810204\end{array}$ $\begin{array}{llll}0 & 0.154405 & 1.932992 & 2.981671\end{array}$ $\begin{array}{llll}0 & -2.248909 & 1.400382 \quad 3.457937\end{array}$ $\begin{array}{llll}\text { C }-0.861874 & 2.620840 & -0.016052\end{array}$ C -1.6747362 .4603351 .122470$ $\begin{array}{llll}\text { C } & 1.878158 & 3.809873 & 0.599278\end{array}$ C $4.128757 \quad 1.271474 \quad 0.024409$ $\begin{array}{llll}\text { C } 4.724200 & 1.288462 & 1.461048\end{array}$ $\begin{array}{llll}\text { C } 4.568517 & 2.507292 & -0.796723\end{array}$ $\begin{array}{llll}\text { C } 4.899521 & 0.129946 & -0.696242\end{array}$ C $2.2023494 .663138-0.659852$ C $2.976814 \quad 3.920476 \quad 1.681409$ $\begin{array}{llll}\text { C } & 2.976814 & 3.920476 & 1.681409 \\ \text { C } & 0.691258 & 4.539038 & 1.283765\end{array}$ C $-1.344883 \quad 3.293378-1.155217$ $\begin{array}{llll}\text { C }-1.344883 & 3.293378 & -1.155217 \\ \text { C }-2.931083 & 3.049945 & 1.172233\end{array}$ C $-2.617074 \quad 3.873381-1.070587$ C -3.3980223 .7750960 .076935$ C $-0.551039 \quad 3.376972-2.450757$ C $-0.6508624 .754216-3.120237$ C $-0.9716132 .283603-3.447409$ H $0.505093 \quad 3.195391-2.206612$ H $-0.451148 \quad 5.574042-2.414583$ H $0.073268 \quad 4.822305-3.946520$ H $-1.650927 \quad 4.917867-3.551038$ H $-0.793751 \quad 1.279515-3.040464$ H $-0.398401 \quad 2.386468-4.382747$ H $-2.042632 \quad 2.374145 \quad-3.693719$ H $-3.5281832 .915706 \quad 2.075030$ $\mathrm{H}-4.377290-4.2539690 .114193$ $\mathrm{H}-4.3772903 .2539690 .114193$ H $-3.003493 \quad 4.424585-1.928880$ $\begin{array}{llll}\text { C } 3.017822 & -1.885104 & 1.126724\end{array}$ C $3.674494-3.116681-1.011092$ $\begin{array}{lll}\text { C } 3.674494 & -3.116681 & 1.011092 \\ \text { C } 3.299006 & -3.160239 & -1.368910\end{array}$ 
C $3.853413-3.737325-0.221736$ $\begin{array}{llll}\text { C } 2.761926 & -1.305400 & 2.512278\end{array}$ C $1.462200-1.863643 \quad 3.114746$ C $3.901564-1.5757103 .505108$ H $2.628899-0.218213 \quad 2.42175$ H $0.574236-1.5428232 .557841$ H $1.496614-2.965302 \quad 3.145210$ H $1.349864-1.490902 \quad 4.144988$ H $4.897324-1.340304 \quad 3.095787$ H $3.908949-2.6317843 .824086$ H $3.747865-0.966432 \quad 4.410702$ $\begin{array}{llll}\text { C } & 1.904047 & -1.412744 & -2.549391\end{array}$ $\begin{array}{llll}\text { C } & 2.404179 & -0.395264 & -3.375766\end{array}$ $\begin{array}{llll}\text { C } & 2.704179 & -0.395264 & -3.375766 \\ \text { H } & 1.011182 & -0.882726 & -2.177182\end{array}$ $\begin{array}{llll}\text { H } & 1.011182 & -0.882726 & -2.177182 \\ \text { H } & 2.901296 & 0.525085 & -2.810058\end{array}$ $\begin{array}{llll}\text { H } & 2.901296 & 0.525085 & -2.810058 \\ \text { H } & 3.665649 & -0.820344 & -3.707694\end{array}$ $\begin{array}{llll}\text { H } & 3.665649 & -0.820344 & -3.707694 \\ \text { H } & 2.128529 & -0.116653 & -4.272961\end{array}$ $\begin{array}{llll}\text { H } & 2.128529 & -0.116653 & -4.272961 \\ \text { H } & 0.893233 & -3.326450 & -2.902902\end{array}$ H $2.247855-3.007479-4.018415$ H $0.721499-2.130351-4.216501$ H $4.054793-3.6011801 .911516$ H $4.389695-4.687459-0.287730$ H $3.384584-3.684896-2.319974$ H $5.9580750 .436382-0.687004$ H $4.849322-0.839942-0.194726$ $\begin{array}{llll}\mathrm{H} & 4.603618 & 0.010905 & -1.744260\end{array}$ $\begin{array}{llll}\text { H } & 5.684006 & 1.831304 & 1.453342\end{array}$ H 4.0758231 .7420572 .216254 4.9285640 .2589911 .779500 H $5.666466 \quad 2.491492-0.870520$ H $4.1720582 .446465-1.823051$ H $4.2904433 .470340-0.374952$ H $3.053837 \quad 4.980144 \quad 1.968308$ H $2.6677313 .357500 \quad 2.575405$ H $3.9729313 .598068 \quad 1.382283$ H $2.7699245 .556397 \quad-0.350934$ H $2.7771724 .145721-1.434825$ H $1.2680125 .012523-1.117843$ H $1.074094 \quad 5.524997 \quad 1.591185$ H $-0.158353 \quad 4.727038 \quad 0.620450$ H $0.354238 \quad 4.0018462 .180161$ C $-0.283472-1.975660-0.011848$ $\begin{array}{llll}\text { C } & -0.283472 & -1.975660 & -0.011848 \\ \text { H } & .555370 & -2.441010 & 0.515913\end{array}$ H $-0.369744-2.351500-1.040148$ $\mathrm{N}-1.5925220 .007059-0.948973$ $\begin{array}{llll}\mathrm{N} & -1.592522 & 0.007059 & -0.948973 \\ \mathrm{C} & -2.706543 & 0.078386 & -1.285946\end{array}$ $\begin{array}{llll}\text { C }-2.706543 & 0.078386 & -1.285946 \\ C & -4.042485 & 0.273686 & -1.687824\end{array}$ $\begin{array}{llll}\text { C } & -4.042485 & 0.273686 & -1.687824 \\ \text { C }-4.887200 & -0.728013 & -1.985239\end{array}$ $\begin{array}{llll}\text { C }-4.887200 & -0.728013 & -1.985239 \\ \text { H }-4.363185 & 1.319232 & -1.717344\end{array}$ $\begin{array}{llll}\mathrm{H} & -4.363185 & 1.319232 & -1.717344 \\ \mathrm{H} & -5.917052 & -0.511452 & -2.272713\end{array}$ H $-4.575733-1.773510-1.939780$ 94

$21 \mathrm{R}=\mathrm{Me} \mathrm{AC} / \mathrm{N}-\mathrm{C}$

Ni $0.015745 \quad 0.008192 \quad-0.083729$ N $1.9626810 .010856-0.089836$ N $0.345772 \quad 1.873735 \quad 0.007190$

$\begin{array}{llll}\text { C } & 1.591694 & 2.272348 & 0.266064\end{array}$

$\begin{array}{llll}\text { C } & 2.583072 & 1.191094 & -0.052164\end{array}$ $\begin{array}{llll}\text { C } & 2.583072 & 1.191094 & -0.052164 \\ \text { C } & 2.545306 & -1.296964 & -0.216775\end{array}$ $\begin{array}{llll}\text { C } & 2.545306 & -1.296964 & -0.216775 \\ \text { S } & -1.150543 & 1.195364 & 2.579699\end{array}$ O $-1.104781 \quad-0.058206 \quad 1.720946$ $\begin{array}{lllll}0 & -1.104781 & -0.058206 & 1.720946 \\ 0 & 0.176361 & 1.558603 & 3.105858\end{array}$ $\begin{array}{llll}0 & 0.176361 & 1.558603 & 3.105858 \\ \mathrm{O} & -2.261151 & 1.159438 & 3.532748\end{array}$ $\begin{array}{llll}\mathrm{O} & -2.261151 & 1.159438 & 3.532748 \\ \mathrm{C} & -0.806045 & 2.695162 & 0.214386\end{array}$ $\begin{array}{llll}\text { C } & -0.806045 & 2.695162 & 0.214386 \\ \text { C }-1.601482 & 2.463016 & 1.350197\end{array}$ $\begin{array}{llll}\text { C } & -1.601482 & 2.463016 & 1.350197\end{array}$ $\begin{array}{llll}\text { C } & 1.951442 & 3.631061 & 0.950531\end{array}$ $\begin{array}{llll}\text { C } & 4.119249 & 1.323681 & -0.370631 \\ \text { C } 4.978126 & 0.707005 & 0.757374\end{array}$ $\begin{array}{llll}\text { C } 4.978126 & 0.707005 & 0.757374\end{array}$ $\begin{array}{llll}\text { C } 4.655766 & 2.733972 & -0.694238\end{array}$ $\begin{array}{llll}\text { C } 4.456358 & 0.544014 & -1.661046\end{array}$ C $2.368436 \quad 4.712459-0.08713$ C $3.019534 \quad 3.3386732 .035210$ $\begin{array}{llll}\text { C } 0.840010 & 4.321647 & 1.784402\end{array}$ C $-1.2106213 .616953-0.772122$ $\begin{array}{llll}\text { C } & -1.210621 & 3.616953 & -0.772122 \\ \text { C }-2.771516 & 3.187439 & 1.543464\end{array}$ $\begin{array}{llll}\text { C } & -2.771516 & 3.187439 & 1.543464 \\ \text { C }-2.392394 & 4.330436 & -0.541875\end{array}$ $\begin{array}{llll}\text { C } & -2.392394 & 4.330436 & -0.541875 \\ \text { C }-3.161888 & 4.133433 & 0.600104\end{array}$ $\begin{array}{llll}\text { C }-3.161888 & 4.133433 & 0.600104 \\ \text { C }-0.429948 & 3.823792 & -2.062971\end{array}$ $\begin{array}{llll}\text { C }-0.429948 & 3.823792 & -2.062971 \\ \text { C }-0.501089 & 5.264261 & -2.583788\end{array}$ $\begin{array}{llll}\text { C } & -0.501089 & 5.264261 & -2.583788 \\ \text { C } & -0.891409 & 2.848811 & -3.159351\end{array}$ $\begin{array}{lllll}\text { C } & -0.891409 & 2.848811 & -3.159351 \\ \text { H } & 0.625819 & 3.597813 & -1.855805\end{array}$ $\begin{array}{cccc}\text { H } & 0.625819 & 3.597813 & -1.855805 \\ \text { H } & -0.266090 & 6.000570 & -1.800841\end{array}$ H $0.214778 \quad 5.396528 \quad-3.409318$ H $-1.500387 \quad 5.498885 \quad-2.982237$ H $-0.725808 \quad 1.805720-2.861380$ H $-0.333684 \quad 3.038173-4.090685$ H $-1.9655442 .981460-3.366712$ H $-3.360000 \quad 2.987087 \quad 2.439371$ H $-4.075680 \quad 4.709764 \quad 0.747804$ H $-2.721668 \quad 5.061477-1.279971$ $\begin{array}{llll}\text { H } & -2.721668 & 5.061477 & -1.279971 \\ \text { C } & 2.891882 & -1.968557 & 0.981152\end{array}$ $\begin{array}{llll}\text { C } & 2.891882 & -1.968557 & 0.981152 \\ \text { C } 2.544138 & -1.984462 & -1.457241\end{array}$ $\begin{array}{llll}\text { C } & 2.544138 & -1.984462 & -1.457241 \\ \text { C } 3.313623 & -3.299782 & 0.893979\end{array}$ C $2.969874-3.314513-1.482726$ C $3.365599-3.974129-0.325419$ $\begin{array}{llll}\text { C } 2.735678 & -1.339249 & 2.357980\end{array}$ $\begin{array}{llll}\text { C } 1.454114 & -1.847441 & 3.035879\end{array}$ C $3.929850-1.602742 \quad 3.286096$ H $2.625944 \quad-0.252504 \quad 2.237622$ H $0.555129-1.5967602 .458734$ H $1.502782-2.940645 \quad 3.175112$ H $1.346337-1.374223 \quad 4.023939$ H $4.892790-1.3313542 .826565$ H $3.981370-2.663305 \quad 3.583639$ H $3.808723-1.0132$ $\begin{array}{llll}\text { H } 3.808723 & -1.013249 & 4.209287\end{array}$ $\begin{array}{llll}\text { C } 2.097434 & -1.360906 & -2.777622\end{array}$ $\begin{array}{llll}\text { C } & 0.780578 & -1.966792 & -3.293292\end{array}$

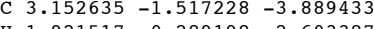
H $1.921517-0.289198-2.603387$ H $4.157509-1.207525-3.576314$ H $3.213309-2.565099-4.222700$ H $2.860432-0.915792-4.764495$ H $-0.050689-1.798298-2.60079$ $\begin{array}{lllll}\text { H } & 0.887217 & -3.053021 & -3.446543\end{array}$ H $0.520696-1.515055-4.263824$ H $3.599071-3.8232821 .808560$ H $3.709902-5.010990-0.367931$ H $2.992199-3.843589-2.436624$ H $5.5026380 .762270-1.926352$ H $4.373663-0.537493-1.533260$ H $3.8237810 .869662-2.498895$ H 6.0409850 .8774790 .520130 $\begin{array}{llll}\mathrm{H} & 4.040985 & 0.877479 & 0.520130\end{array}$ H $4.820701-0.3767010 .817686$ $\begin{array}{llll}\mathrm{H} & 4.820701 & -0.376701 & 0.817686 \\ \mathrm{H} & 5.733756 & 2.623239 & -0.888120\end{array}$ $\begin{array}{llll}\text { H } & 5.733756 & 2.623239 & -0.888120 \\ \text { H } & 4.204648 & 3.122641 & -1.618486\end{array}$ $\begin{array}{lllll}\mathrm{H} & 4.204648 & 3.122641 & -1.61848 \\ \mathrm{H} & 4.550047 & 3.479398 & 0.095119\end{array}$ $\begin{array}{llll}\text { H } & 4.550047 & 3.479398 & 0.095119 \\ \text { H } & 3.331374 & 4.291915 & 2.490564\end{array}$ $\begin{array}{llll}\mathrm{H} & 3.331374 & 4.291915 & 2.490564 \\ \mathrm{H} & 2.557860 & 2.718792 & 2.819545\end{array}$ H $3.915398 \quad 2.830302 \quad 1.682935$ H $3.047461 \quad 5.437455 \quad 0.390766$ H $2.856677 \quad 4.329681-0.986105$ H $1.4743165 .264116-0.409564$ H $1.331649 \quad 5.180142 \quad 2.271950$ H $0.018847 \quad 4.728187 \quad 1.184452$ H $0.449097 \quad 3.662833 \quad 2.569076$ C $-1.727710 \quad 0.103602-1.039325$ H $-1.561387-0.280244-2.054702$ $\mathrm{H}-2.426312-0.523250-0.472024$ $\begin{array}{llll}\mathrm{H} & -2.426312 & -0.523250 & -0.472024 \\ \mathrm{H} & -2.048941 & 1.150357 & -1.038721\end{array}$ $\mathrm{N}-0.224228-1.793511-0.183855$ $\begin{array}{llll}\mathrm{N} & -0.224228 & -1.793511 & -0.183855 \\ \mathrm{C} & -0.369947 & -2.940633 & -0.051068\end{array}$ $\begin{array}{llll}\text { C } & -0.369947 & -2.940633 & -0.051068 \\ \text { C }-0.445661 & -4.350412 & 0.063002\end{array}$ $\begin{array}{llll}\text { C }-0.445661 & -4.350412 & 0.063002\end{array}$ $\begin{array}{llll}C & -1.601232 & -5.007704 & 0.247878\end{array}$ H $0.515923-4.869540 \quad-0.005698$ $\begin{array}{lllll}\text { H } & -1.607365 & -6.095681 & 0.327817\end{array}$ $\begin{array}{llll}\text { H } & -2.554975 & -4.481065 & 0.324559\end{array}$ 99

$21 \mathrm{R}=\mathrm{n}-\mathrm{Pr} \mathrm{ET} / \mathrm{pi}$

Ni $0.008191-0.0305220 .009663$ N $2.016673-0.0250210 .050108$ C $2.675833 \quad 1.251243 \quad 0.157530$ $\begin{array}{lll}\text { C } 2.646448 & -1.181762 & -0.054594\end{array}$ C $4.089948-1.4632700 .513925$

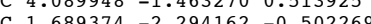
$\begin{array}{llll}C & 1.689374 & -2.294162 & -0.502269\end{array}$ $\begin{array}{llll}C & 2.164332 & -3.536109 & -1.306608\end{array}$ N $0.441327-1.972533-0.285349$ $\begin{array}{llll}\text { C } & -0.701287 & -2.773319 & -0.587625\end{array}$ C $-1.468607-2.418714-1.714678$ $\begin{array}{llll}C & -2.652438 & -3.083861 & -2.003331\end{array}$ $\begin{array}{llll}\text { C }-3.101766 & -4.094380 & -1.15610\end{array}$ $\begin{array}{llll}\text { C }-2.368282 & -4.411775 & -0.02041\end{array}$ $\begin{array}{llll}\text { C } & -1.167026 & -3.765494 & 0.297753\end{array}$ H $-3.209697-2.783556-2.89118$ H $-4.030511-4.621945-1.374202$ H $-2.739157-5.1882840 .649606$ S $-0.940473-1.065839-2.804423$ $\begin{array}{lllll}0 & -0.858991 & 0.104484 & -1.831853\end{array}$ o $0.381245-1.444743-3.331714$ $\begin{array}{lllll}0 & -2.020897 & -0.878647 & -3.771421\end{array}$ C $-0.449108-4.147061 \quad 1.582988$ $\begin{array}{llll}C & -0.449108 & -4.147061 & 1.582988\end{array}$ $\begin{array}{llll}\text { C }-0.193805 & -5.660021 & 1.678300\end{array}$ $\begin{array}{llll}C & -1.237732 & -3.687656 & 2.821273\end{array}$ H $0.294028-6.0584850 .777620$ H $-1.137882-6.2103091 .813136$ H $0.443573-5.882978 \quad 2.548062$ H $-2.210707-4.2023992 .869822$ H $-0.680383-3.930815 \quad 3.739897$ H $-1.434556 \quad-2.607599 \quad 2.807398$ H $0.524261-3.633585 \quad 1.594674$ C $3.0771091 .838091-1.063039$ C $3.6979933 .096392-1.029371$ C $3.888271 \quad 3.780687 \quad 0.162528$ $\begin{array}{llll}\text { C } 3.410217 & 3.210257 & 1.358342\end{array}$ C $2.788487 \quad 1.960823 \quad 1.379301$ H $4.028800 \quad 3.545335-1.967905$ H $4.377719 \quad 4.757155 \quad 0.176893$ H 3.5293573 .7624552 .293706 $\begin{array}{llll}\text { H } & 3.529357 & 3.762455 & 2.293706 \\ \text { C } 2.866307 & 1.170505 & -2.415349\end{array}$ C $2.1242262 .085966-3.401014$ C $4.1991370 .713753-3.028121$ H $2.2311700 .284553-2.280900$

H $4.8567961 .575558-3.229062$ H $4.0180570 .190971-3.979815$ H $4.7435710 .030759-2.359766$ H $2.7271582 .966125 \quad-3.674879$ H $1.899018 \quad 1.525132-4.320393$ H $1.170698 \quad 2.436065 \quad-2.982052$ C $2.284862 \quad 1.447227 \quad 2.726845$ C $3.404389 \quad 1.192513 \quad 3.755836$ $\begin{array}{llll}\text { C } 1.296110 & 2.439122 & 3.369190\end{array}$ H $1.7575910 .499892 \quad 2.547246$ $\begin{array}{llll}\text { H } & 1.757591 & 0.499892 & 2.547246\end{array}$ $\begin{array}{llll}H & 4.100025 & 0.398548 & 3.457307\end{array}$ H 3.9923662 .1086803 .924809 H $2.957194 \quad 0.899985 \quad 4.719253$ H 0.5078852 .7598042 .675935 H $0.822816 \quad 1.981721 \quad 4.251966$ H $1.822948 \quad 3.344575 \quad 3.709289$ $\begin{array}{llll}\text { C } 3.800907 & -2.152434 & 1.876936\end{array}$ H $4.755985-2.382618 \quad 2.377210$ H $3.223134-1.4804812 .528589$ H $3.233346-3.0869641 .776339$ C $4.949881-0.217952 \quad 0.822464$ H $5.1649120 .376444 \quad-0.076072$ H $5.911114-0.585518 \quad 1.216082$ H $4.526442 \quad 0.444498 \quad 1.574304$ C $5.085246-2.317266-0.314706$ н $4.723933-3.264496-0.704159$ H $5.480551-1.732147-1.158439$ $\begin{array}{llll}H & 5.480551 & -1.732147 & -1.158439\end{array}$ $\begin{array}{llllll}H & 5.936235 & -2.538077 & 0.348871\end{array}$ C $3.060365-3.002334-2.463440$ $\begin{array}{llll}\text { H } 2.402665 & -2.660812 & -3.274422 \\ \text { H } 3.703183 & -3.815386 & -2.838056\end{array}$ $\begin{array}{llll}\text { H } & 3.703183 & -3.815386 & -2.838056 \\ \mathrm{C} & .696679 & -2.155394 & -2.196519\end{array}$ $\begin{array}{llll}\text { C } 2.824581 & -4.606646 & -0.398958\end{array}$ H $3.260832-5.386369-1.044392$ H $2.049405 \quad-5.0860190 .215107$ $\begin{array}{lllll}\text { H } 3.605147 & -4.257442 & 0.278267\end{array}$ $\begin{array}{llll}\text { C } 1.072065 & -4.331212 & -2.059944\end{array}$ H $0.505363-3.695645 \quad-2.749634$ H $1.612644-5.076272-2.667580$ H $0.386446-4.881289-1.404618$ C $-0.194483 \quad 1.942289-0.072189$ $\begin{array}{lllll}C & -1.570630 & 2.559817 & -0.177290\end{array}$ H $0.3666992 .078766-1.008490$ $\begin{array}{llll}\mathrm{H} & 0.366699 & 2.078766 & -1.008490\end{array}$ $\begin{array}{llll}\text { H } 0.382710 & 2.352687 & 0.765579\end{array}$ $\begin{array}{cccc}C & -1.732969 & -0.300015 & 1.024302\end{array}$ $\begin{array}{llll}c & -0.679156 & -0.072294 & 1.914302\end{array}$ $\mathrm{H}-2.078105-1.3130520 .803559$ $\mathrm{H}-2.443752 \quad 0.489480 \quad 0.786902$ $\begin{array}{lllll}\text { H } & -0.579566 & 0.897888 & 2.401732\end{array}$ H $-0.183533 \quad-0.906478 \quad 2.419303$ C $-1.467453 \quad 4.034055 \quad-0.608960$ H $-2.099569 \quad 2.519790 \quad 0.789794$ H $-2.172070 \quad 2.013554 \quad-0.920231$ H $-2.469208 \quad 4.491863-0.657415$ H $-0.854775 \quad 4.6162230 .098585$ H $-1.004740 \quad 4.116243-1.605068$ 100

$21 \mathrm{R}=\mathrm{Me} \mathrm{AC} / \mathrm{pi}$

$\mathrm{Ni}-0.003465-0.014848-0.081835$

N $2.025032-0.008615-0.029849$

C $2.6914731 .273985-0.009432$

$\begin{array}{llll}\text { C } 2.633686 & -1.174448 & -0.031338\end{array}$ $\begin{array}{llll}\text { C } 4.060898 & -1.432387 & 0.585704\end{array}$ C $1.669433-2.311014-0.453856$ C $2.179884-3.581814-1.182781$ N $0.418694-1.989272-0.279630$ C $-0.713594-2.792311-0.64560$ C $-1.377264-2.459172-1.844543$ C $-2.526042-3.132061-2.237250$ $\begin{array}{llll}\text { C }-3.049080 & -4.133890 & -1.423856\end{array}$ $\begin{array}{llll}\text { C }-2.428396 & -4.424054 & -0.217534\end{array}$ $\begin{array}{llll}\text { C }-1.266367 & -3.766301 & 0.210036\end{array}$ H $-2.996200-2.846077-3.178343$ H $-3.948755-4.672075-1.722879$ $\begin{array}{lllll}\mathrm{H} & -2.858708 & -5.189810 & 0.428395\end{array}$ S $-0.761658-1.127291-2.904770$ $\begin{array}{llll}S & -0.761658 & -1.127291 & -2.904770\end{array}$ $\begin{array}{lllll}0 & -0.774137 & 0.060756 & -1.941964 \\ 0 & 0.602202 & -1.503605 & -3.309278\end{array}$ $\begin{array}{llll}0 & 0.602202 & -1.503605 & -3.309278 \\ 0 & -1.753595 & -0.945053 & -3.961856\end{array}$ $0-1.75355-0.945053-3.961856$ $\begin{array}{llll}\text { C }-0.697298 & -4.134845 & 1.570809\end{array}$ $\begin{array}{llll}\text { C }-0.358279 & -5.632085 & 1.669677\end{array}$ $\begin{array}{llll}\text { C }-1.682587 & -3.776696 & 2.696929\end{array}$ H $0.274145 \quad-5.978547 \quad 0.840846$ H $-1.274802-6.242781 \quad 1.659651$ H $0.165369-5.836063 \quad 2.616400$ H $-2.591184 \quad-4.395772 \quad 2.625693$ H $-1.214904 \quad-3.956585 \quad 3.676340$ н $-1.987323-2.722404 \quad 2.663261$ H $0.221976-3.554014 \quad 1.735389$ 
$\begin{array}{llll}\text { C } 2.937931 & 0.991963 & -2.566944\end{array}$ C $2.172422 \quad 1.807226-3.620389$ $\begin{array}{llll}\text { C } 4.292977 & 0.540212 & -3.133875\end{array}$ H $2.3325410 .096261 \quad-2.378030$ H $4.920471 \quad 1.406862 \quad-3.399367$ H $4.139100-0.061771-4.042571$ H $4.857760-0.066934-2.410805$ H $2.7378242 .697199-3.939063$ H $1.990171 \quad 1.179321-4.505201$ $\begin{array}{llll}\text { H } & 1.195120 & 2.134769 & -3.239412\end{array}$ $\begin{array}{llll}\text { H } & 1.195120 & 2.134769 & -3.239412 \\ \text { C } & 2.293044 & 1.665268 & 2.537068\end{array}$ $\begin{array}{llll}\text { C } & 2.293044 & 1.665268 & 2.537068 \\ \text { C } & 3.416165 & 1.515072 & 3.599364\end{array}$ $\begin{array}{llll}\text { C } & 3.416165 & 1.515072 & 3.599364 \\ \text { C } & 1.279633 & 2.678179 & 3.103284\end{array}$ $\begin{array}{llll}\text { C } & 1.279633 & 2.678179 & 3.103284 \\ \text { H } & 1.802675 & 0.688939 & 2.444370\end{array}$ $\begin{array}{lllllllll}\text { H } & 1.802675 & 0.688939 & 2.444370\end{array}$ H $4.129840 \quad 0.7148123 .365909$ H $3.9795512 .456564 \quad 3.705739$ H $2.957947 \quad 1.277639 \quad 4.572838$ $\begin{array}{lllll}\text { H } & 0.492133 & 2.943128 & 2.385385\end{array}$ H $0.808645 \quad 2.268875 \quad 4.010485$ H $1.7855293 .614320 \quad 3.387949$ C $3.728497 \quad-2.017179 \quad 1.987911$ H $4.672432-2.224032 \quad 2.518381$ H $3.139517-1.3045612 .581548$ H $3.147528-2.947533 \quad 1.944562$ $\begin{array}{llll}\text { C } 4.922385 & -0.173173 & 0.819318\end{array}$ H $5.1662380 .348859-0.116178$ $\begin{array}{lll}5.166238 & 0.348859-0.116178\end{array}$ H $5.869272-0.515498 \quad 1.26620$ $\begin{array}{llll}\text { H } & 4.484899 & 0.545593 & 1.507536 \\ \text { C } & 5.070744 & -2.353173 & -0.149167\end{array}$ $\begin{array}{llll}\text { C } & 5.070744 & -2.353173 & -0.149167 \\ \text { H } & 4.713239 & -3.324661 & -0.477075\end{array}$ $\begin{array}{llll}\text { H } & 4.713239 & -3.324661 & -0.477075 \\ \text { H } 5.499559 & -1.836171 & -1.020484\end{array}$ $\begin{array}{lllll}\text { H } & 5.499559 & -1.836171 & -1.020484 \\ \text { H } 5.896372 & -2.530641 & 0.557638\end{array}$ $\begin{array}{llll}\text { H } 5.896372 & -2.530641 & 0.557638 \\ \text { C } 3.140911 & -3.106880 & -2.313178\end{array}$ $\begin{array}{llll}\text { C } 3.140911 & -3.106880 & -2.313178 \\ \text { H } 2.535506 & -2.848136 & -3.191918\end{array}$ $\begin{array}{llll}\text { H } 2.535506 & -2.848136 & -3.191918 \\ \text { H } 3.832876 & -3.922129 & -2.578410\end{array}$ $\begin{array}{llll}\text { H } & 3.832876 & -3.922129 & -2.578410 \\ \text { H } & 3.732095 & -2.220536 & -2.069921\end{array}$ C $2.784358-4.598485-0.178570$ H $3.261702-5.410389-0.751087$ H $1.972548-5.042662 \quad 0.412713$ H $3.516762-4.2054790 .527283$ C $1.131654-4.417714-1.954329$ $\begin{array}{llll}\mathrm{H} & 0.604476 & -3.822038 & -2.707770\end{array}$ $\begin{array}{llll}\mathrm{H} & 1.707580 & -5.190917 & -2.490119\end{array}$ $\begin{array}{llll}\text { H } & 0.410055 & -4.935892 & -1.312574\end{array}$ $\begin{array}{llll}\text { C }-0.197692 & 1.957836 & -0.276501\end{array}$ H $0.3589082 .049889-1.219673$ $\begin{array}{llll}\mathrm{H} & 0.384263 & 2.397701 & 0.542259 \\ \mathrm{C} & -1.578832 & 2.554602 & -0.396165\end{array}$ $\begin{array}{lllll}C & -1.726978 & -0.260871 & 0.888401\end{array}$ $\begin{array}{llll}\text { C }-0.721106 & 0.115731 & 1.809985\end{array}$ H $-2.035731-1.304190 \quad 0.792383$ H $-2.4663600 .477088 \quad 0.584120$ H $-0.704363 \quad 1.146845 \quad 2.171475$ C $-0.059549-0.789855 \quad 2.687289$ N $0.524508-1.4803413 .429835$ $\begin{array}{llll}\text { C } & -1.490217 & 4.021719 & -0.855792\end{array}$ H $-2.112466 \quad 2.529214 \quad 0.569132$ H $-2.170443 \quad 1.986914-1.131184$ H $-2.497603 \quad 4.464426-0.920230$ $\begin{array}{llll}\mathrm{H} & -2.497603 & 4.464426 & -0.920230\end{array}$ $\mathrm{H}-0.890795 \quad 4.624847-0.154980$ H

$21 \mathrm{R}=\mathrm{n}-\mathrm{Pr} \quad \mathrm{AC} / \mathrm{N}-\mathrm{t}$

Ni $-0.020443-0.0269510 .045108$ $\mathrm{N} 1.890988-0.010513 \quad 0.029273$ $\mathrm{N} \quad 0.348987 \quad 1.897967 \quad 0.006496$ $\begin{array}{llll}\text { C } 1.578177 & 2.318278 & 0.210029\end{array}$ C $2.551195 \quad 1.1493490 .069566$ C $2.544932-1.279456-0.059273$ S $-1.1498791 .478403 \quad 2.573277$ $\begin{array}{llll}0 & -0.966129 & 0.121781 & 1.906275\end{array}$ $\begin{array}{llll}0 & 0.120445 \quad 2.040912 \quad 3.059502\end{array}$ $\begin{array}{lllll}0 & -2.279217 & 1.470681 & 3.504262\end{array}$ C $-0.865374 \quad 2.634775 \quad 0.024665$ $\begin{array}{llll}C & -1.686986 & 2.496382 & 1.159427\end{array}$ C $-1.686983 \quad 2.496382 \quad 1.159427$ $\begin{array}{llll}\text { C } 4.118923 & 1.258827 & -0.043777\end{array}$ c 4.7732981 .2625231 .367327

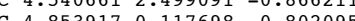
$\begin{array}{llll}C & 4.853917 & 0.117698 & -0.802095\end{array}$ $\begin{array}{llll}C & 2.228599 & 4.664920 & -0.664721\end{array}$ C 3.0061623 .8952931 .667767 C $0.726851 \quad 4.554001 \quad 1.285160$ C $-1.335458 \quad 3.305421-1.122475$ C $-2.9429293 .087777 \quad 1.192888$ C $-2.6082693 .885805-1.054834$ $\begin{array}{lllll}\text { C }-3.401843 & 3.797441 & 0.084747\end{array}$ $\begin{array}{lllll}\text { C }-0.523773 & 3.397344 & -2.406486\end{array}$ C $-0.629365 \quad 4.774834-3.075190$ C $-0.912123 \quad 2.301163-3.41240$ H $0.530534 \quad 3.230001 \quad-2.146732$ H $-0.4565975 .595432-2.363290$ H $-0.456597 .595432-2.363290$ H $0.1112784 .85510-3.885551$ $\mathrm{H}-1.6222594 .925343-3.526834$ $\mathrm{H}-0.7146731 .29633-3.008336$ $\begin{array}{llll}\mathrm{H} & -0.332711 & 2.421589 & -4.341853 \\ \mathrm{H} & -1.982825 & 2.368969 & -3.667374\end{array}$
H $-3.544732 \quad 2.9680192 .094570$ H $-4.381851 \quad 4.275499 \quad 0.107285$ н $-2.985194 \quad 4.431104 \quad-1.920834$ C $3.045635-1.8920331 .111333$ $\begin{array}{llll}\text { C } 2.543359 & -1.948141 & -1.307435\end{array}$ $\begin{array}{llll}\text { C } 3.693391 & -3.126012 & 0.965284\end{array}$ $\begin{array}{llll}\text { C } 3.216802 & -3.165267 & -1.395498\end{array}$ $\begin{array}{llll}\text { C } 3.817485 & -3.744204 & -0.274325\end{array}$ C $2.859763-1.304382 \quad 2.504739$ C $1.567548-1.81$ $\begin{array}{llll}\text { C } 1.567548 & -1.816745 & 3.161075\end{array}$ 093.452782 $\begin{array}{llll}\text { H } 2.757291 & -0.214190 & 2.414118\end{array}$ $\begin{array}{lllll}\text { H } & 0.670732 & -1.455697 & 2.643680\end{array}$ H $1.559970-2.9189003 .185742$ H $1.512780-1.446128 \quad 4.196821$ H $5.014850-1.391348 \quad 3.003057$ $\begin{array}{lllll}\text { H } 4.025395 & -2.658568 & 3.772617\end{array}$ H $3.928501-0.989391 \quad 4.363086$ C $1.784189-1.410785-2.518027$ C $1.260408-2.535326-3.420770$ $\begin{array}{llll}\text { C } 2.553098 & -0.393265 & -3.373814\end{array}$ H $0.907728-0.877962-2.113456$ H $2.7711730 .527009-2.815911$ H $3.500923-0.818297-3.742919$ H $1.943012-0.114506-4.247892$ $40.75898-3.323462-2.84004$ $\begin{array}{llll}H & 2.070590 & -3.001143 & -4.00418\end{array}$ H $0.539046-2.121757-4.141909$ H $4.113403-3.6122611 .847185$ H $4.347931-4.695636-0.36428$ H $3.261434-3.688531-2.35008$ H $5.9143570 .416210-0.830945$ H $4.816005-0.855978-0.30691$ H $4.5176120 .009571-1.838909$ H $5.739467 \quad 1.791913 \quad 1.318656$ H 4.1649771 .7256922 .149764 H $4.977860 \quad 0.230103 \quad 1.676027$ H $5.634206 \quad 2.469323-0.986107$ H $4.1015132 .459535-1.875912$ H $4.2950443 .459157-0.417940$ H $3.092786 \quad 4.949005 \quad 1.973455$ H 2.702779 H 2.7027793 .3176802 .554521 .

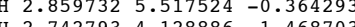
H $2.742793 \quad 4.128886-1.468703$ 1.301242 $5.077773-1.082532$ 1.1221125 .5397591 .577678 H -0.1289264 .7425800 .629804$ $\begin{array}{lllll}\text { H } & 0.395082 & 4.028791 & 2.190518\end{array}$ C $-0.304400-2.021384 \quad 0.128091$ H $-0.274948-2.341034-0.925499$ н $0.593951-2.382023 \quad 0.645492$ C $-1.561732-2.500206 \quad 0.822997$ $\begin{array}{llll}\mathrm{N} & -1.568986 & -0.020417 & -0.935132\end{array}$ C $-2.6291890 .042727-1.417990$ $\begin{array}{llll}\text { C }-3.919831 & 0.177827 & -1.962259\end{array}$ C $-4.557539-0.827981-2.583682$ H $-4.3837211 .159716-1.827883$ H $-5.567054-0.687180-2.972523$ \begin{tabular}{l}
$-5.567054-0.687180-2.972523$ \\
\hline
\end{tabular} $\begin{array}{llll}H & -4.093271 & -1.808970 & -2.709163\end{array}$ c $-1.625508-4.0367170 .817653$ H $-2.465372-2.1026350 .335524$ $-1.586860-2.1361211 .859480$ H $-2.530048-4.3863791 .339937$ $\begin{array}{llll}\text { H } & -1.647105 & -4.434099 & -0.209574 \\ \text { H } & -0.750067 & -4.470299 & 1.325198\end{array}$ $\mathrm{H}-0$

$21 \mathrm{R}=\mathrm{n}-\mathrm{Pr} \quad \mathrm{AC} / \mathrm{N}-\mathrm{C}$

Ni $-0.007564 \quad 0.001618-0.024499$ N $1.947011-0.005771-0.011726$ N $0.341860 \quad 1.878831 \quad 0.003548$ $\begin{array}{llll}\text { C } 1.592014 & 2.271045 & 0.262474\end{array}$ $\begin{array}{llll}\text { C } 2.572218 & 1.174668 & -0.019994\end{array}$ c $2.537485-1.313005-0.088456$ $\begin{array}{lllll}\text { S }-1.286981 & 1.291946 & 2.536975\end{array}$ o $-1.209352 \quad 0.0172501 .711538$ $\begin{array}{lllll}0 & -1.209352 & 0.017250 & 1.711538\end{array}$ $0.0097101 .646038 \quad 3.135847$ $\begin{array}{lllll}0 & -2.454637 & 1.290127 & 3.420515\end{array}$ $\begin{array}{llll}C & -0.798094 & 2.728532 & 0.150837\end{array}$ C 1.6510712 .5346770 .253893 c 1.9585973 .6333680 .941693 $\begin{array}{llll}\text { C } 4.102140 & 1.293786 & -0.371039\end{array}$ $\begin{array}{lllll}C & 4.994900 & 0.705151 & 0.745869\end{array}$ $\begin{array}{llll}C & 4.629256 & 2.696066 & -0.736362\end{array}$ C $4.4004910 .484285-1.652356$ $\begin{array}{llll}\text { C } 2.381217 & 4.723444 & -0.082910\end{array}$ $\begin{array}{llll}\text { C } 3.027927 & 3.337981 & 2.023675\end{array}$ C $0.843527 \quad 4.318998 \quad 1.774132$ C $-1.143510 \quad 3.642928-0.867547$ $\begin{array}{llll}\text { C }-2.816599 & 3.281319 & 1.378940\end{array}$ $\begin{array}{lllll}C & -2.323106 & 4.378801 & -0.704617\end{array}$ C -3.1491894 .2148260 .401636$ C $-0.3108833 .823141-2.129906$ C $-0.310883-3.823141-2.129906$ C $-0.3003615 .270710-2.63894$ C $-0.7848862 .886228-3.253567$ H $-0.071166 \quad 5.991509-1.840422$

H $0.455421 \quad 5.380374 \quad-3.431574$ H $-1.271158 \quad 5.548654-3.077829$ H $-0.684292 \quad 1.832690-2.965154$ H $-0.1889173 .057892-4.164449$ H $-1.843797 \quad 3.075878-3.492803$ H $-3.447355 \quad 3.108551 \quad 2.251492$ H $-4.059390 \quad 4.807747 \quad 0.496411$ H $-2.6049335 .100732-1.470841$ C $2.910871-1.921854 \quad 1.134679$ $\begin{array}{lll}\text { C } 2.528549 & -2.061592 & -1.293648\end{array}$ $\begin{array}{lll}\text { C } 3.353487 & -3.252289 & 1.106001\end{array}$ C $2.976296-3.384094-1.260983$ $\begin{array}{llll}\text { C } 2.976296 & -3.384094 & -1.260983 \\ \text { C } 3.400330 & -3.980458 & -0.080249\end{array}$ $\begin{array}{llll}\text { C } & 3.400330 & -3.980458 & -0.080249\end{array}$ $\begin{array}{llll}\text { C } & 2.767193 & -1.222622 & 2.478316 \\ \text { C } 1.460289 & -1.644348 & 3.167156\end{array}$ C $3.944033-1.4811493 .428610$ $\begin{array}{llll}\text { H } 2.702172 & -0.140019 & 2.302488\end{array}$ H $0.576118-1.3765192 .574709$ H $1.458002-2.732609 \quad 3.347853$ H $1.368280-1.1281104 .135076$ H $4.919097-1.275628 \quad 2.959467$ H $3.950230-2.5234413 .788496$ H $3.843443-0.834690 \quad 4.315373$ $\begin{array}{lll}\text { C } 2.050010 & -1.514826 & -2.637223\end{array}$ $\begin{array}{llll}\text { C } 0.746130 & -2.183671 & -3.107274\end{array}$ $\begin{array}{llll}\text { C } 3.094514 & -1.696995 & -3.755542\end{array}$

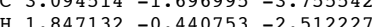
$\begin{array}{llll}\mathrm{H} & 1.847132 & -0.440753 & -2.51222\end{array}$ $\begin{array}{llll}\mathrm{H} & 4.093374 & -1.340245 & -3.475812\end{array}$ $\begin{array}{llll}H & 3.183512 & -2.758704 & -4.034065\end{array}$ $\begin{array}{llll}\text { H } & 2.770960-1.152101 & -4.656211 \\ \mathrm{H} & -0.082043-2.014060-2.411070\end{array}$ H $-0.082043-2.014060-2.411070$ H $0.886745-3.271457-3.216026$ н $0.459505-1.784058-4.09308$ н $3.661844-3.7262542 .040020$ H $3.762778-5.012016-0.079440$ $\begin{array}{llll}\text { H } & 2.993767 & -3.958321 & -2.188913\end{array}$ н $5.4362880 .698581-1.959015$ H $4.326199-0.593412-1.492119$ H $3.737287 \quad 0.784559 \quad-2.476205$ H $6.049668 \quad 0.859379 \quad 0.465037$ H $4.832714 \quad 1.175436 \quad 1.724772$ H $4.833828 \quad-0.3748120 .849967$ H $5.6988332 .581039-0.970315$ H $5.6988332 .581039-0.970315$ $\begin{array}{lllll}\text { H } & 4.142314 & 3.073249 & -1.647167\end{array}$

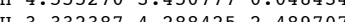
\begin{tabular}{l}
3.3323878 .2884252 .489707 \\
\hline
\end{tabular} 2.5735382 .7064532 .803267 H 3.9280312 .8420401 .663339 H 3.0365655 .4556900 .416727 $\begin{array}{lllll}\text { H } 2.897348 & 4.356217 & -0.972111\end{array}$ H $1.486995 \quad 5.263956-0.421388$ H $1.332506 \quad 5.1705892 .276061$ H $0.030336 \quad 4.735427 \quad 1.169656$ H $0.440495 \quad 3.652994 \quad 2.546624$ $\begin{array}{llll}\text { C }-1.672396 & 0.101964 & -1.158462\end{array}$ $\begin{array}{llll}\text { C }-2.842506 & -0.794771 & -0.817751\end{array}$ H $-1.9532751 .158839-1.065536$ н $-1.257449-0.103379-2.158579$ N $-0.231257-1.805820 \quad-0.014961$ $\begin{array}{llll}\mathrm{N}-0.311093 & -2.937585 & 0.244655\end{array}$ $\begin{array}{llll}\text { C }-0.324745 & -4.348768 & 0.493362\end{array}$ $\begin{array}{llll}\text { C }-1.387256 & -4.978263 & 1.017637\end{array}$ H $0.601952-4.871007 \quad 0.230793$ H $-1.357174-6.055140 \quad 1.189605$ H $-2.297245-4.437988 \quad 1.287814$ $\begin{array}{lll}\text { C }-4.100737 & -0.352240 & -1.583272\end{array}$ $\begin{array}{lllll}\mathrm{H} & -3.040751 & -0.746649 & 0.264124\end{array}$ H $-2.620228-1.843622-1.069217$ H $-4.948413-1.016439-1.352354$ H $-4.3858790 .673455-1.303124$ H

$21 \mathrm{R}=\mathrm{CHCNE}$ ET/pi

Ni $0.055401-0.106709-0.138398$

N $2.114472-0.107269-0.045991$

$\begin{array}{llll}\mathrm{C} & 2.760266 & 1.175408 & 0.095605\end{array}$

$\begin{array}{llll}\text { C } & 2.760266 & 1.175408 & 0.095605 \\ \text { C } 2.711797 & -1.271007 & -0.070629\end{array}$

$\begin{array}{llll}\text { C } & 2.711797 & -1.271007 & -0.070629 \\ \text { C } & 4.186359 & -1.562922 & 0.376178\end{array}$

$\begin{array}{llll}\text { C } & 4.186359 & -1.562922 & 0.376178 \\ \text { C } & 1.696435 & -2.417416 & -0.327840\end{array}$ C $2.058493-3.715006-1.104523$ N $0.486546-2.053418-0.015362$ C $-0.695257-2.853458-0.088969$ C $-1.569703-2.675038-1.178132$ C $-2.789758-3.338558-1.210220$ C $-3.160502-4.172529-0.14712$ C $-2.312295-4.304703 \quad 0.945426$ $\begin{array}{llll}C & -1.080522 & -3.642429 & 1.012926\end{array}$ H $-3.452647-3.181328-2.061375$ H $-4.119207-4.694392-0.16795$ H $-2.620130-4.929477 \quad 1.785218$ S -1.181498 $-1.623992-2.620926$ 
H $0.697345 \quad-5.697652 \quad 1.662944$ H $-0.695421-5.8843552 .756754$ H $0.865162-5.307022 \quad 3.403420$ н $-1.872391-3.8361723 .725569$ H $-0.323295 \quad-3.265296 \quad 4.386900$ H $-1.311591-2.193656 \quad 3.354085$ H $0.677749-3.187266 \quad 2.137219$ C $3.1248731 .830553-1.102216$ $\begin{array}{lllll}\text { C } 3.731511 & 3.099836 & -1.013927\end{array}$ c 3.9313 $\begin{array}{llll}\text { C } 3.931358 & 3.715133 & 0.214910\end{array}$ $\begin{array}{llll}\text { C } 3.505592 & 3.072191 & 1.379136\end{array}$ $\begin{array}{llll}\text { C } & 2.908816 & 1.811495 & 1.354721 \\ \mathrm{H} & 4.030461 & 3.610266 & -1.931210\end{array}$ $\begin{array}{lllll}\text { H } & 4.393456 & 4.703472 & 0.271521\end{array}$ $\begin{array}{lllll}\text { H } & 4.393456 & 4.703472 & 0.271521 \\ \mathrm{H} & 3.629035 & 3.577264 & 2.338603\end{array}$ $\begin{array}{llll}\text { H } & 3.629035 & 3.577264 & 2.338603 \\ \text { C } & 2.909753 & 1.213909 & -2.478324\end{array}$ $\begin{array}{llll}\text { C } & 2.909753 & 1.213909 & -2.478324 \\ \text { C } & 2.389088 & 2.232525 & -3.507347\end{array}$ $\begin{array}{llll}\text { C } & 2.389088 & 2.232525 & -3.507347 \\ \text { C } & 4.192983 & 0.567891 & -3.021115\end{array}$ $\begin{array}{llll}\text { C } & 4.192983 & 0.567891 & -3.021115 \\ \text { H } & 2.149751 & 0.422442 & -2.387413\end{array}$ H $4.9937001 .319254-3.120290$ H $4.006993 \quad 0.126410-4.012733$ H $4.559460-0.226812-2.359663$ H $3.182295 \quad 2.929827-3.821184$ H $2.0401491 .700688-4.405683$ H $1.554815 \quad 2.828965 \quad-3.111472$ c $2.468428 \quad 1.2245732 .695922$ $\begin{array}{llll}\text { C } 2.468428 & 1.224573 & 2.695922 \\ \text { C } 3.636087 & 0.914794 & 3.655690\end{array}$ $\begin{array}{llll}\text { C } 3.636087 & 0.914794 & 3.655690\end{array}$ $\begin{array}{llll}C & 1.528808 & 2.188692 & 3.448839\end{array}$ $\begin{array}{llll}\text { H } & 1.923887 & 0.290529 & 2.494148\end{array}$ $\begin{array}{lllll}\text { H } & 4.312127 & 0.126528 & 3.304516\end{array}$ H $4.238873 \quad 1.820354 \quad 3.828353$ H $3.229473 \quad 0.597062 \quad 4.628923$ H $0.790823 \quad 2.662581 \quad 2.791416$ H $1.012736 \quad 1.651509 \quad 4.259794$ H $2.111518 \quad 3.000009 \quad 3.914155$ C $4.020518-2.292817 \quad 1.737603$ H $5.015320-2.5606632 .129810$ H $3.533939-1.626742 \quad 2.464255$ H $3.419882-3.2084261 .676126$ C $5.071993-0.326894 \quad 0.646702$ H $5.2241840 .286860-0.250842$ H $6.057704-0.709259 \quad 0.957896$ H 4.7090050 .3205531 .442370 C $5.087178-2.375705-0$. $\begin{array}{llll}\text { C } 5.087178 & -2.375705 & -0.594382\end{array}$ H $4.669734-3.280045-1.026174$ H $5.420387-1.734232-1.423263$ H $5.988248-2.661788-0.028822$ C $2.669824-3.209646-2.442208$ H $1.852804-2.844956-3.084181$ H $3.166016-4.051177-2.953055$ H $3.394613-2.396152-2.337928$ C $2.943041-4.715725-0.322104$ H $3.257728-5.508418-1.022382$ H $2.336535 \quad-5.197008 \quad 0.461996$ H $3.841180-4.3246620 .157800$ C $0.881848-4.611754-1.553609$ н $0.184987-4.101736-2.230398$ $\begin{array}{llll}\mathrm{H} & 1.336968 & -5.433598 & -2.133884 \\ \mathrm{H} & 0.332652 & -5.068217 & -0.718222\end{array}$ $\begin{array}{llll}\mathrm{H} & 0.332652 & -5.068217 & -0.718222\end{array}$ $\begin{array}{llll}C & -0.307579 & 1.757896 & -0.817999\end{array}$ $\begin{array}{llll}C & -1.649732 & 1.975657 & -1.534902\end{array}$ $\begin{array}{llll}\mathrm{H} & 0.516410 & 1.731317 & -1.543914\end{array}$ $\begin{array}{llll}\text { C } & -0.082934 & 2.796308 & 0.141540\end{array}$ $\begin{array}{lllll}C & -1.714791 & -0.080572 & 0.863684\end{array}$ $\begin{array}{llll}C & -0.639520 & 0.188435 & 1.721691\end{array}$ H $-2.159097 \quad-1.0773610 .808953$ $\mathrm{H}-2.364086 \quad 0.729673 \quad 0.533780$ H $-0.450442 \quad 1.207963 \quad 2.057948$ H $-0.223592-0.6037362 .350314$ $\begin{array}{llll}\mathrm{N} & -0.007886 & 3.669532 & 0.918051\end{array}$ $\begin{array}{llll}\mathrm{C} & -1.553610 & 3.114088 & -2.558804\end{array}$ H $-2.423267 \quad 2.234003-0.795854$ H $-1.956082 \quad 1.051862 \quad-2.042342$ H $-2.539434 \quad 3.285644 \quad-3.019394$ $\begin{array}{llll}H & -1.223581 & 4.053062 & -2.086214\end{array}$ H $-0.8474232 .857051-3.363169$ 101

$21 \mathrm{R}=\mathrm{CHCNEt} \mathrm{AC} / \mathrm{pi}$

$\mathrm{Ni}-0.001259-0.0107790 .000920$ $\mathrm{N} 2.125090-0.0079960 .014299$ $\begin{array}{llll}\text { C } 2.943550 & 1.200971 & 0.031142\end{array}$ C $2.606555-1.216246 \quad 0.122629$ $\begin{array}{llll}\text { C } 3.932399 & -1.650414 & 0.797462\end{array}$ C $1.612290-2.321031-0.327797$ C $2.019197-3.404739-1.394055$ $\begin{array}{llll}\mathrm{N} & 0.397340 & -2.037651 & 0.024099\end{array}$ C $-0.757339-2.875452-0.125349$ C $-1.710531-2.569712-1.114236$ C $-2.888071-3.302689-1.214117$ C $-3.138186-4.340034 \quad-0.322807$ $\begin{array}{llll}\text { C }-2.218355 & -4.611674 & 0.680783\end{array}$ C $-1.028822-3.887421 \quad 0.822944$ H $-3.603950-3.045537-1.995478$ H $-4.057268-4.921120-0.399443$ $\begin{array}{llll}\text { H } & -4.057268 & -4.921120 & -0.399443 \\ \mathrm{H} & -2.432600 & -5.405416 & 1.396721\end{array}$ S $-1.488954-1.276445-2.371093$ $0-0.227898-0.527560-1.914926$
O $-1.191950-1.977138-3.624722$ $\begin{array}{llll}0 & -2.713138 & -0.478043 & -2.31387\end{array}$ $\begin{array}{llll}\text { C }-0.121894 & -4.215294 & 1.994435\end{array}$ $\begin{array}{llll}\text { C } 0.528179 & -5.633348 & 1.844238\end{array}$ C $-0.870246 \quad-4.134384 \quad 3.332251$ H $1.082848 \quad-5.730634 \quad 0.898579$ H $-0.246596-6.419091 \quad 1.862761$ H $1.222571-5.814524 \quad 2.682113$ H $-1.626393-4.9304043 .416625$ $\mathrm{H}-0.159853-4.250163 \quad 4.164071$ $\mathrm{H}-0.159853-4.250163 \quad 4.164071$ $\begin{array}{llll}\mathrm{H} & -1.372759 & -3.164976 & 3.454579\end{array}$ $\begin{array}{llll}\mathrm{H} & 0.687786 & -3.476347 & 2.028649 \\ \mathrm{C} & 3.503147 & 1.578517 & -1.218987\end{array}$ c $3.5031471 .578517-1.218987$ $\begin{array}{llll}\text { C } 4.315926 & 2.724416 & -1.28584\end{array}$ $\begin{array}{llll}C & 4.537360 & 3.530005 & -0.181759\end{array}$ C $3.896155 \quad 3.200267 \quad 1.017690$ C $3.0936892 .063351 \quad 1.153655$ $\begin{array}{lllll}\text { H } & 4.761313 & 2.991847 & -2.246480\end{array}$ H $5.172799 \quad 4.416069-0.247750$ H $4.031768 \quad 3.8484851 .885717$ $\begin{array}{llll}\text { C } 3.204877 & 0.886354 & -2.540426\end{array}$ $\begin{array}{llll}\text { C } 2.498481 & 1.855624 & -3.505721\end{array}$ $\begin{array}{lllll}\text { C } 4.462110 & 0.329959 & -3.224606\end{array}$ H $2.507627 \quad 0.064325-2.349800$ H $5.154216 \quad 1.144040-3.493176$ H $4.184256-0.189552-4.154868$ H $4.184256-0.189552-4.154868$ $\begin{array}{llll}\text { H } 5.013280 & -0.373770 & -2.586942\end{array}$ $\begin{array}{llll}\text { H } & 3.190141 & 2.635959 & -3.861208\end{array}$ H $2.1259281 .305667-4.383670$ $\begin{array}{llll}\text { H } & 1.648843 & 2.355225 & -3.024260\end{array}$ C 2.4545321 .8739752 .521300 C $3.4705281 .909048 \quad 3.695477$ C $1.4148132 .974517 \quad 2.792896$ H $1.957060 \quad 0.897583 \quad 2.540038$ H $4.348858 \quad 1.272857 \quad 3.530994$ H $3.826100 \quad 2.937459 \quad 3.867380$ H $2.965974 \quad 1.575855 \quad 4.616126$ H $0.673674 \quad 3.077826 \quad 1.993176$ H $0.884330 \quad 2.772675 \quad 3.736889$ H $1.912723 \quad 3.951938 \quad 2.895505$ C $3.621557 \quad-2.907470 \quad 1.654615$ H $4.570364-3.325248 \quad 2.028306$ H $3.012076-2.6071852 .521373$ $\begin{array}{llll}\text { H } 3.012076-2.607185 & 2.521373\end{array}$ H $3.087108-3.706282 \quad 1.136739$ C $4.434880-0.6406471 .834593$ H 4.8192440 .2849291 .391836 $\begin{array}{llll}\text { H } 5.263834 & -1.118597 & 2.381738\end{array}$ H $3.648825-0.4160882 .562778$ C $5.112445-1.822836-0.184856$ H $4.911418-2.455837-1.055897$ H $5.424553-0.831568-0.549372$ $\begin{array}{llll}\text { H } & 5.962757 & -2.261085 & 0.365908\end{array}$ C $2.517019-2.560412-2.591061$ H $1.723329-1.884593-2.942498$ H $2.769049-3.249981-3.412928$ H $3.413045-1.975969-2.352738$ C $3.107072-4.436342-1.031089$ H $3.326275-5.004801-1.950574$ H $2.741995-5.160014-0.284494$ H $2.741995-5.160014-0.284494$ H $4.052995-4.021231-0.673240$ $\begin{array}{llll}\text { C } & 0.855049 & -4.253398 & -1.943467\end{array}$ H $0.094471-3.655851-2.462049$ $\mathrm{H} 1.292524-4.925820-2.700233$ H $0.376165-4.884913-1.182710$ C $-0.3323791 .949470-0.450111$ C $-0.858917 \quad 2.205662-1.866465$ $\begin{array}{llll}\text { H } & 0.713400 & 2.291296 & -0.331359\end{array}$ C $-1.1603792 .638469 \quad 0.498452$ $\begin{array}{llll}C & -1.647576 & -0.163352 & 1.107667\end{array}$ C $-0.546856 \quad 0.209456 \quad 1.926989$ H $-2.002983 \quad-1.196352 \quad 1.097548$ H $-2.392735 \quad 0.594650 \quad 0.862511$ H $-0.486920 \quad 1.245565 \quad 2.268803$ C $0.166653-0.689877 \quad 2.769359$ N 0.8216967 .3603883 .469986 1.8685893 .1969261 .24 $\begin{array}{llll}\mathrm{C} & -0.866909 & 3.705418 & -2.201992\end{array}$ $\mathrm{H}-1.8724341 .788709-1.960212$ H $-0.231143 \quad 1.662778-2.579246$ H $-1.2136043 .847827-3.238972$ $\begin{array}{lllll}\mathrm{H} & -1.541036 & 4.265032 & -1.533175 \\ \mathrm{H} & 0.141715 & 4.144068 & -2.112531\end{array}$ $\begin{array}{ll}\mathrm{H} & 0.14 \\ 101 & \end{array}$

$21 \mathrm{R}=\mathrm{CHCNE} \mathrm{AC} / \mathrm{N}$

Ni $0.002659 \quad-0.000551 \quad 0.003741$ $\begin{array}{llll}\mathrm{N} & 1.914388 & 0.001457 & -0.001072\end{array}$ $\mathrm{N} 0.362733 \quad 1.897615 \quad 0.001498$ $\begin{array}{llll}\text { C } 1.581441 & 2.324571 & 0.24866\end{array}$ $\begin{array}{llll}\text { C } 2.562142 & 1.151358 & 0.168175\end{array}$ C $2.542056-1.281030-0.177716$ S $-0.7008591 .549948 \quad 2.740380$ \begin{tabular}{llll}
\hline & -0.546714 & 0.179844 & 2.105745
\end{tabular} $\begin{array}{lllll}0 & 0.599097 & 2.207727 & 2.980778\end{array}$ $\begin{array}{llll}0 & 0.599097 & 2.207727 & 2.980778 \\ 0 & -1.656851 & 1.544054 & 3.848702\end{array}$ $\begin{array}{llll}0 & -1.656851 & 1.544054 & 3.848702\end{array}$ C $-0.870492 \quad 2.601094 \quad 0.147189$ C 1.9029773 .8480160 .439864

$\begin{array}{llll}\text { C } 4.112097 & 1.265760 & 0.367065\end{array}$ $\begin{array}{llll}\text { C } 4.363827 & 1.449324 & 1.897475\end{array}$ $\begin{array}{llll}\text { C } 4.756078 & 2.398340 & -0.475174\end{array}$ C $4.9830220 .051752-0.047808$ C $2.306527 \quad 4.417695-0.949190$ $\begin{array}{llll}\text { C } 2.955750 & 4.166610 & 1.528446\end{array}$ C $0.714321 \quad 4.722312 \quad 0.915936$ C $-1.528834 \quad 3.184228 \quad-0.948292$ C $-2.773326 \quad 3.017714 \quad 1.586438$ C $-2.808543 \quad 3.706008 \quad 0.725082$ $\begin{array}{llll}C & -2.808543 & 3.706008 & -0.725082\end{array}$ $\begin{array}{llll}C & -3.424358 & 3.639771 & 0.521594\end{array}$ $\begin{array}{llll}C & -0.892817 & 3.278892 & -2.326438\end{array}$ $\begin{array}{llll}C & -0.964478 & 4.706149 & -2.891509\end{array}$ $\begin{array}{llll}C & -1.512261 & 2.291691 & -3.328709\end{array}$ $\begin{array}{lllll}\mathrm{H} & 0.167169 & 3.004648 & -2.219641\end{array}$ H $-0.6006315 .456074 \quad-2.173738$ H $-0.360233 \quad 4.779635-3.808955$ H $-1.998729 \quad 4.974872 \quad-3.157547$ н $-1.341279 \quad 1.248899-3.030157$ H $-1.0658862 .440345-4.324878$ H $-2.5987932 .454586-3.418019$ H $-3.2356112 .917955 \quad 2.569276$ H $-4.417942 \quad 4.066955 \quad 0.662845$ H $-3.334927 \quad 4.183978-1.552740$ $\begin{array}{llll}\text { C } 2.873681 & -2.075172 & 0.941556\end{array}$ C $2.661307-1.771532-1.501222$ $\begin{array}{llll}\text { C } 2.661307 & -1.771532 & -1.501222 \\ \text { C } 3.409326 & -3.346295 & 0.703797\end{array}$ $\begin{array}{llll}\text { C } 3.409326 & -3.346295 & 0.703797\end{array}$ $\begin{array}{llll}C & 3.222754 & -3.035604 & -1.678796\end{array}$ $\begin{array}{llll}\text { C } 3.610323 & -3.825279 & -0.585671\end{array}$ C $2.686371-1.6233662 .383468$ $\begin{array}{lll}C & 1.483249-2.308220 & 3.043770\end{array}$ $\begin{array}{llll}\text { C } 3.925698 & -1.902264 & 3.251212\end{array}$ H $2.483372-0.5432232 .390735$ H $0.540335-2.0034822 .580376$ H $1.582946-3.4043302 .989036$ H $1.429907 \quad-2.018678 \quad 4.104964$ H $4.866124-1.576538 \quad 2.778113$ H $4.021588-2.979331 \quad 3.470337$ H $3.824168-1.380575 \quad 4.217331$ $\begin{array}{llll}\text { C } 2.165894 & -0.982183 & -2.709621\end{array}$ $\begin{array}{llll}\text { C } 1.866032 & -1.864137 & -3.925590\end{array}$ $\begin{array}{llll}\text { C } 3.121253 & 0.142370 & -3.136665\end{array}$ H $1.217095 \quad-0.507826-2.401167$ H $3.2550570 .891288-2.401167$ H $3.2550570 .891288-2.347484$ $\begin{array}{llll}\text { H } & 4.109539 & -0.266114 & -3.405000\end{array}$ H $2.7158330 .657091-4.022250$ $\mathrm{H} 1.205589-2.702918-3.668918$ H $2.792772-2.251264-4.379061$ H $1.357052-1.259853 \quad-4.691399$ H $3.682544 \quad-3.971656 \quad 1.555069$ H $4.040051-4.816817-0.748427$ H $3.339689-3.433263-2.685250$ H $6.026166 \quad 0.355124 \quad 0.137575$ H $4.805958-0.8520640 .537751$ H $4.896143-0.196249-1.113371$ H $5.265546 \quad 2.061834 \quad 2.059141$ H $3.526323 \quad 1.908126 \quad 2.434378$ H $4.542091 \quad 0.469400 \quad 2.355475$ $\begin{array}{lllll}\text { H } 5.828180 & 2.422101 & -0.226334\end{array}$ H $4.6792862 .168614-1.549018$ $\begin{array}{lllll}\mathrm{H} & 4.6796796 & 2.168614 & -1.549018 \\ \mathrm{H} & 3.078398 & 3.398931 & -0.312606\end{array}$ $\begin{array}{llll}\mathrm{H} & 4.366798 & 3.398931 & -0.31260 \\ \mathrm{H} & 3.078379 & 5.262148 & 1.548175\end{array}$ $\begin{array}{llll}\text { H } & 3.078379 & 5.262148 & 1.548175 \\ \mathrm{H} & 2.568461 & 3.853997 & 2.511166\end{array}$ $\begin{array}{llll}\text { H } & 2.568461 & 3.853997 & 2.511166 \\ \mathrm{H} & 3.947043 & 3.737693 & 1.390689\end{array}$ $\begin{array}{llll}\text { H } & 3.947043 & 3.737693 & 1.390689 \\ \text { H } & 2.874031 & 5.352154 & -0.808415\end{array}$ $\begin{array}{llll}\text { H } & 2.874031 & 5.352154 & -0.808415 \\ \text { H } & 2.909823 & 3.740757 & -1.564385\end{array}$ $\begin{array}{lllll}\mathrm{H} & 2.909823 & 3.740757 & -1.564385 \\ \mathrm{H} & 1.404652 & 4.662854 & -1.525237\end{array}$ $\begin{array}{llll}\text { H } & 1.404652 & 4.662854 & -1.525237 \\ \text { H } & 1.114384 & 5.741273 & 1.039218\end{array}$ H $-0.109634 \quad 4.792874 \quad 0.201969$ H $0.333577 \quad 4.384646 \quad 1.889071$ C $-0.375038-2.013333-0.026188$ $\begin{array}{llll}\text { H } & 0.583472 & -2.451356 & 0.285823\end{array}$ C $-1.507368 \quad-2.443035 \quad 0.921431$ $\begin{array}{lll}\text { C }-0.665725 & -2.402820 & -1.377115\end{array}$ N $-1.6957420 .090423-0.672013$ C $-2.8000410 .084141-1.039563$ $\begin{array}{llll}\text { C } & -2.800041 & 0.084141 & -1.039563 \\ \text { C } & -4.130760 & 0.075274 & -1.505942\end{array}$ $\begin{array}{lll}\text { C }-4.130760 & 0.075274 & -1.505942 \\ \text { C }-4.578294 & -0.868750 & -2.352043\end{array}$ $\begin{array}{llll}\text { C } & -4.578294 & -0.868750 & -2.352043 \\ \mathrm{H} & -4.775464 & 0.873055 & -1.128718\end{array}$ $\begin{array}{llll}\mathrm{H} & -4.775464 & 0.873055 & -1.128718 \\ \mathrm{H} & -5.617277 & -0.860073 & -2.686371\end{array}$ $\begin{array}{llll}\mathrm{H} & -5.617277 & -0.860073 & -2.686371 \\ \mathrm{H} & -3.917678 & -1.659786 & -2.717034\end{array}$ $\begin{array}{llll}\mathrm{H} & -3.917678 & -1.659786 & -2.717034 \\ \mathrm{~N} & -1.001135 & -2.706209 & -2.457890\end{array}$ $\begin{array}{llll}\mathrm{N} & -1.001135 & -2.706209 & -2.457890 \\ \mathrm{C} & -1.523478 & -3.961369 & 1.128468\end{array}$ $\begin{array}{llll}\mathrm{C} & -1.523478 & -3.961369 & 1.128468 \\ \mathrm{H} & -2.476494 & -2.121626 & 0.512073\end{array}$ $\begin{array}{llll}\mathrm{H} & -2.476494 & -2.121626 & 0.512073 \\ \mathrm{H} & -1.387944 & -1.922364 & 1.880953\end{array}$ $\begin{array}{llll}\mathrm{H} & -1.387944 & -1.922364 & 1.880953 \\ \mathrm{H} & -2.346747 & -4.244830 & 1.802058\end{array}$ H $-1.660768 \quad-4.490637 \quad 0.172748$ H $-0.580985 \quad-4.309793 \quad 1.577493$ 
$\begin{array}{llll}\text { H } & 4.323928 & -0.314798 & -0.772779\end{array}$ H $3.942482-1.865432-1.596558$ H $4.139293-1.7921850 .185220$ $\begin{array}{llll}\text { C } 1.347427 & -2.099772 & -0.553086\end{array}$ $\begin{array}{llll}\text { C } 1.775156 & -3.476298 & -0.94136\end{array}$ H $2.257316-3.440216-1.932228$ H $2.512819-3.887292-0.234154$ H $0.913167-4.152833-0.995521$ $\mathrm{N} 0.133903-1.737131-0.230030$ C $-0.925683-2.649222-0.003748$ C $-2.133840-2.386653-0.683242$ $\begin{array}{llll}\text { C }-0.809572 & -3.687787 & 0.950686\end{array}$ $\begin{array}{llll}C & -0.809572 & -3.687787 & 0.950686 \\ \text { C }-3.184705 & -3.289343 & -0.510362\end{array}$ C $-1.897473-4.557637 \quad 1.081376$ C $-3.061668-4.3892720 .336852$ B $-2.342104-1.028165-1.529428$ F $-3.677310-0.799803-1.861669$ F $-1.967046 \quad 0.107276-0.544969$ $\begin{array}{llll}\text { F }-1.492796 & -0.877555 & -2.631848\end{array}$ C $0.347201-3.788167 \quad 1.933589$ $\begin{array}{llll}\text { C } 0.859583 & -5.222165 & 2.118655\end{array}$ C $-0.066623-3.175297 \quad 3.282828$ H $1.183025-3.173054 \quad 1.577737$ H $1.111944-5.695469 \quad 1.157008$ H $1.759620-5.2175592 .752977$ H $0.108757-5.854973 \quad 2.618090$ $\mathrm{H}-0.340014 \quad-2.117633 \quad 3.158092$ $\begin{array}{llll}\mathrm{H} & -0.340014 & -2.117633 & 3.158092 \\ \mathrm{H} & -0.925272 & -3.719012 & 3.709988\end{array}$ $\begin{array}{lllll}\text { H } & -0.925272 & -3.719012 & 3.709988 \\ \text { H } & 0.775888 & -3.220506 & 3.990074\end{array}$ H $-4.121917-3.097788-1.035271$ H $-3.888286-5.092199 \quad 0.455082$ $\begin{array}{llll}\text { H }-1.838676 & -5.373219 & 1.803360\end{array}$ $\begin{array}{llll}\text { C } 2.940319 & 2.172871 & -1.063191\end{array}$ $\begin{array}{llll}\text { C } 3.151429 & 1.431351 & 1.295411\end{array}$ C $3.787097 \quad 3.250641-0.778915$ C 3.9853582 .5343551 .520378 C $4.307508 \quad 3.436858 \quad 0.502725$ $\begin{array}{llll}\text { C } 2.387948 & 1.970793 & -2.464108\end{array}$ $\begin{array}{llll}\text { C } 3.486542 & 1.505480 & -3.433270\end{array}$ C $1.696308 \quad 3.225519-3.013076$ H $1.626102 \quad 1.177415-2.410912$ н $4.2654952 .279946-3.533887$ H $3.062904 \quad 1.311127-4.432188$ H. $3.0629042 .311127-4.432188$ $\begin{array}{lllll}\text { H } 3.973752 & 0.584644 & -3.078994\end{array}$ $7.569490-2.328877$ H $1.2373403 .004219-3.990650$ $\begin{array}{llll}\text { H } 2.414745 & 4.049586 & -3.155384\end{array}$ $\begin{array}{llll}B & 2.787799 & 0.395222 & 2.526287\end{array}$ F $3.402207 \quad 0.855483 \quad 3.721414$ $\begin{array}{llll}\text { F } & 1.379529 & 0.310183 & 2.704384 \\ \text { F } & 3.287314 & -0.923676 & 2.227262\end{array}$ $\begin{array}{llll}\text { F } & 3.287314 & -0.923676 & 2.227262 \\ \text { H } & 4.035873 & 3.961854 & -1.571764\end{array}$ H $4.035873 \quad 3.961854 \quad-1.571764$ H $4.958987 \quad 4.2925200 .704650$ $\begin{array}{lllll}\text { H } & 4.379201 & 2.672333 & 2.529658 \\ \text { C } & -0.294248 & 2.103018 & -0.05310\end{array}$ $\begin{array}{lllll}\text { H }-0.975524 & 2.176465 & 0.813786\end{array}$ H $-0.8096332 .431300 \quad-0.970327$ H $0.632550 \quad 2.658308 \quad 0.131401$

$\begin{array}{llll}22 & \mathrm{R}=\mathrm{Me} \mathrm{Et} / \mathrm{pi} & & \\ -0.062067 & 0.05754 & 0.081718\end{array}$ $\begin{array}{lll}-0.062067 & 0.05754 & 0.081718 \\ 1.913504 & 0.063334 & 0.022951\end{array}$ $\begin{array}{lll}1.913504 & 0.063334 & 0.022951 \\ 2.721112 & 1.249314 & -0.021513\end{array}$ $\begin{array}{lll}2.721112 & 1.249314 & -0.021513 \\ 2.478136 & -1.083389 & 0.268756\end{array}$ $\begin{array}{lll}2.478136 & -1.083389 & 0.268756 \\ 3.939786 & -1.310723 & 0.456914\end{array}$ $\begin{array}{lll}3.939786 & -1.310723 & 0.456914 \\ 4.517504 & -0.41427 & 0.200081\end{array}$ $\begin{array}{lll}4.517504 & -0.41427 & 0.200081 \\ 4.282692 & -2.161425 & -0.151567\end{array}$ $\begin{array}{lll}4.282692 & -2.161425 & -0.15156 \\ 4.127988 & -1.536364 & 1.519724\end{array}$ $\begin{array}{lllll}1.538424 & -2.210241 & 0.365766\end{array}$ $\begin{array}{llll}2.028913 & -3.616504 & 0.302947\end{array}$ $2.534012-3.777433-0.663598$ $\begin{array}{lll}2.756378 & -3.809594 & 1.106851\end{array}$ $\begin{array}{llll}1.1966 & -4.327201 & 0.374345\end{array}$ $\begin{array}{llll}0.292998 & -1.860272 & 0.452217\end{array}$ $\begin{array}{llll}-0.763728 & -2.819098 & 0.370875\end{array}$ $\begin{array}{llll}-1.327362 & -2.987547 & -0.908011\end{array}$ $\begin{array}{llll}-1.255834 & -3.449844 & 1.524409\end{array}$ $-2.416953-3.856769-1.005435$ $-2.348821-4.3115211 .363639$

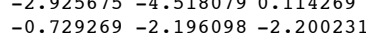
$-0.729269-2.196098-2.200231$
$-1.549267-2.359902-3.329922$ $\begin{array}{lll}-1.549267 & -2.359902 & -3.329922 \\ -0.68005 & -0.74483 & -1.894332\end{array}$ $\begin{array}{lll}-0.68005 & -0.74483 & -1.894332 \\ 0.602371 & -2.597251 & -2.481102\end{array}$ $\begin{array}{lll}-0.655002 & -3.215432 & 2.900285\end{array}$ $-0.079401-4.5083663 .498084$ $\begin{array}{llll}-1.672258 & -2.589228 & 3.866239\end{array}$ $\begin{array}{llll}0.175394 & -2.501317 & 2.790011\end{array}$ $\begin{array}{llll}0.661807 & -4.967028 & 2.826693\end{array}$ $\begin{array}{lll}-0.877224 & -5.248161 & 3.674119\end{array}$ $\begin{array}{lll}0.409362 & -4.297811 & 4.462628\end{array}$ $-2.516878-3.2741544 .046534$ $\begin{array}{llll}-2.516878 & -3.274154 & 4.046534 \\ -2.076585 & -1.648558 & 3.465613\end{array}$ $-2.076585-1.6485583 .465613$ $-1.193224-2.3729684 .834353$ $-3.780005-5.1906110 .016676$ $\begin{array}{llll}-3.780005 & -5.190611 & 0.016676 \\ -2.757507 & -4.822977 & 2.237259\end{array}$

\begin{abstract}
$\begin{array}{lll}2.993079 & 1.7936 & -1.292933\end{array}$ $\begin{array}{lll}3.135952 & 1.838375 & 1.186418\end{array}$ $\begin{array}{lll}3.721244 & 2.98449 & -1.344339\end{array}$ $\begin{array}{llll}3.864926 & 3.034476 & 1.06343\end{array}$ $\begin{array}{llll}4.155735 & 3.611595 & -0.169079\end{array}$ $\begin{array}{llll}2.558312 & 1.084123 & -2.563961\end{array}$ $\begin{array}{llll}2.044286 & 2.043895 & -3.648283\end{array}$ $\begin{array}{llll}3.692707 & 0.209594 & -3.12204\end{array}$ $\begin{array}{lll}1.728613 & 0.408282 & -2.307212\end{array}$ $\begin{array}{lll}1.274552 & 2.719578 & -3.246043\end{array}$ $\begin{array}{lll}1.274552 & 2.719578 & -3.246043 \\ 2.85947 & 2.657963 & -4.06545\end{array}$ $\begin{array}{lll}2.85947 & 2.657963 & -4.06545 \\ 1.603611 & 1.465166 & -4.475502\end{array}$ $\begin{array}{lll}1.603611 & 1.465166 & -4.475502 \\ 4.030953 & -0.526063 & -2.377486\end{array}$ $\begin{array}{lll}4.030953 & -0.526063 & -2.377486 \\ 4.558918 & 0.833317 & -3.400117\end{array}$ $\begin{array}{lll}4.558918 & 0.833317 & -3.400117 \\ 3.350123 & -0.340961-4.012593\end{array}$ $\begin{array}{lll}2.800293 & 1.332713 & 2.725129\end{array}$ $\begin{array}{lll}1.641443 & 2.020088 & 3.192778\end{array}$ $\begin{array}{lll}2.564768 & -0.078723 & 2.817362\end{array}$ $\begin{array}{lll}3.90752 & 1.662695 & 3.557496\end{array}$ $\begin{array}{lll}3.949267 & 3.435748 & -2.313472 \\ 4.71827 & 4.548428 & -0.227348\end{array}$ $\begin{array}{llll}4.71827 & 4.548428 & -0.227348 \\ 4.208793 & 3.510624 & 1.985396\end{array}$ $\begin{array}{llll}-0.259931 & 1.871134 & -0.555803\end{array}$ $\begin{array}{llll}-0.191192 & 1.705403 & -1.643031\end{array}$ $\begin{array}{lll}0.5675 & 2.482635 & -0.171905\end{array}$ $\begin{array}{lll}-1.22962 & 2.318362 & -0.296122\end{array}$ $\begin{array}{lll}-0.805277 & 0.625934 & 1.842087\end{array}$ $\begin{array}{lll}-1.840942 & 0.157465 & 1.017785\end{array}$ $\begin{array}{lll}-1.840942 & 0.157465 & 1.017785 \\ -0.626883 & 1.690982 & 2.00127\end{array}$ $\begin{array}{lll}-0.322233 & -0.016658 & 2.582643\end{array}$ $\begin{array}{lll}-2.509204 & 0.858644 & 0.511895 \\ -2.224382 & -0.863196 & 1.110435\end{array}$ $22 \mathrm{R}=\mathrm{Me} \mathrm{AC} / \mathrm{pi}$
\end{abstract} 0

Ni $0.059750 \quad 0.006612-0.149322$ N $2.029087 \quad 0.005505-0.129484$ $\begin{array}{llll}\text { C } 2.861326 & 1.172540 & -0.011718\end{array}$ $\begin{array}{llll}\text { C } 2.581817 & -1.167983 & -0.027612\end{array}$ $\begin{array}{llll}\text { C } 4.034322 & -1.424168 & 0.186979\end{array}$ H $4.390080-2.238198-0.461046$ H $4.630191-0.5199330 .012235$ H $4.178872-1.7187301 .240688$ $\begin{array}{llll}\text { C } 1.642343 & -2.296852 & -0.138988\end{array}$ $\begin{array}{lll}\text { C } 1.642343-2.296852 & -0.138988\end{array}$ $\begin{array}{llll}\text { C } 2.143123 & -3.676660 & -0.39567\end{array}$ H. $1.310860-4.383064-0.500103$ $10.716496-3.680498-1.336947$ $\begin{array}{llll}\text { H } 2.812971 & -4.003272 & 0.415165\end{array}$ C $0.393928-1.962775-0.066119$ $\begin{array}{llll}C & -0.652301 & -2.888936 & -0.366216\end{array}$ $\begin{array}{llll}C & -1.147036 & -2.813122 & -1.681875\end{array}$ B $-0.472423-1.808547-2.771082$ F $-1.207922-1.760717-3.961325$ F $0.881152-2.150534-3.019957$ F $-0.454269-0.425588-2.205743$ C $-2.225486-3.643227-1.996178$ H $-2.629048-3.601072-3.009529$ C $-2.788119-4.497050-1.045354$ H $-3.635001-5.132861-1.309329$ C $-2.277324-4.5310130 .248439$ $\begin{array}{llll}\mathrm{H} & -2.277324 & -4.531013 & 0.248439\end{array}$ $\begin{array}{llll}C-1.197051 & -3.721269 & 0.623892\end{array}$ C $-0.661179-3.7587972 .045469$ H $0.172430-3.0428982 .112265$

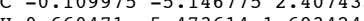
H $0.660471-5.473614 \quad 1.693424$ $\begin{array}{lllll}\mathrm{H} & -0.913847 & -5.900412 & 2.402673\end{array}$ H $0.335035 \quad-5.129670 \quad 3.414932$ C $-1.721347 \quad-3.324991 \quad 3.06906$ H $-2.105513 \quad-2.318136 \quad 2.85035$ H $-1.289059-3.313887 \quad 4.08205$ H $-2.575667 \quad-4.020843 \quad 3.06897$ C $3.266969 \quad 1.591913 \quad 1.264765$ B $2.841258 \quad 0.938606 \quad 2.720823$ F $3.9032631 .146770 \quad 3.641197$ F $2.568110-0.469752 \quad 2.644699$ $\begin{array}{llll}F & 2.568110 & -0.469752 & 2.644699\end{array}$ $\begin{array}{llll}F & 1.669461 & 1.600955 & 3.191913\end{array}$ $\begin{array}{llll}\text { C } 4.078797 & 2.760725 & 1.295998\end{array}$ 4.4240523 .1075222 .274181 $\begin{array}{llll}\text { C } & 4.435755 & 3.444749 & 0.139152 \\ \text { H } 5.060093 & 4.341341 & 0.192273\end{array}$ $\begin{array}{llll}\text { H } 5.060093 & 4.341341 & 0.192273 \\ \text { C } 3.985586 & 2.982260 & -1.113722\end{array}$ $\begin{array}{llll}\text { C } 3.985586 & 2.982260 & -1.113722 \\ \mathrm{H} & 4.264002 & 3.525176 & -2.019965\end{array}$ $\begin{array}{llll}\text { H } & 4.264002 & 3.525176 & -2.019965 \\ \text { C } 3.189925 & 1.842332 & -1.210179\end{array}$ $\begin{array}{llll}\text { C } 3.189925 & 1.842332 & -1.210179 \\ \text { C } 2.748484 & 1.313384 & -2.564981\end{array}$ $\begin{array}{llll}\text { C } & 2.748484 & 1.313384 & -2.564981 \\ \text { H } & 1.891331 & 0.642303 & -2.408161\end{array}$ H $1.8913310 .642303-2.408161$ C $2.2910052 .422506-3.521210$ H $1.835917 \quad 1.973779 \quad-4.417917$ H $1.5465923 .078098-3.045125$ H $3.1369623 .047359-3.851359$ $\begin{array}{lllll}\text { C } 3.856245 & 0.472201 & -3.219354\end{array}$ H $3.501528 \quad 0.053076-4.174156$ H $4.748914 \quad 1.091406-3.410451$ Н $4.156253-0.365882-2.572933$ C $-0.059957 \quad 1.914041-0.469464$ H $0.173544 \quad 1.937209-1.544366$ H $0.697088 \quad 2.426410 \quad 0.138180$ H $-1.0691372 .302031-0.283456$ $\begin{array}{llll}\text { C }-1.720249 & -0.085714 & 0.832982\end{array}$ C $-0.631973 \quad 0.263919 \quad 1.676231$ H $-2.043173-1.128801 \quad 0.744397$ H $-0.124967 \quad-0.498188 \quad 2.271964$ $\begin{array}{llll}\text { C }-2.755780 & 0.836393 & 0.504041\end{array}$ H $-0.511908 \quad 1.281598 \quad 2.049666$ 70 $22 \mathrm{R}=\mathrm{Me} \mathrm{AC} / \mathrm{N}$

$\mathrm{Ni} 0.011554 \quad 0.026433 \quad-0.020640$ $\mathrm{N} 1.916397 \quad 0.009708 \quad-0.042651$ $\begin{array}{llll}\mathrm{N} & 1.916397 & 0.009708 & -0.042651\end{array}$ $\begin{array}{llll}\text { C } 2.470561 & -1.166640 & 0.062896\end{array}$ $\begin{array}{llll}C & 2.470561 & -1.166640 & 0.062896\end{array}$ $\begin{array}{llll}\text { C } & .934472 & -1.417533 & 0.150550 \\ \text { H } 4.217007 & -2.267809 & -0.487544\end{array}$ $\begin{array}{llll}\mathrm{H} & 4.217007 & -2.267809 & -0.487544 \\ \mathrm{H} & 4.513284 & -0.527113 & -0.122363\end{array}$ $\begin{array}{llll}\text { H } & 4.513284 & -0.527113 & -0.122363\end{array}$ $\begin{array}{llll}\text { H } & 4.178252 & -1.657537 & 1.199781\end{array}$ $\begin{array}{llll}\text { C } 1.518348 & -2.288003 & 0.151434\end{array}$ $\begin{array}{llll}\text { C } 1.997559 & -3.693501 & 0.242017\end{array}$ $\begin{array}{llll}\text { H } & 1.181170 & -4.366762 & 0.531932\end{array}$ H $2.345363-4.016986-0.752947$ H $2.826280 \quad-3.7724890 .961246$ $\begin{array}{llll}\text { N } 0.279466 & -1.905445 & 0.171380\end{array}$ C $-0.788254 \quad-2.8523390 .280950$ $\begin{array}{llll}\text { C }-1.215718 & -3.536243 & -0.870684\end{array}$ B $-0.575430-3.293459-2.370710$ F $-1.388674-3.950076-3.333687$ F $0.752844-3.830337-2.438046$ F $-0.518016-1.898600-2.652310$ C $-2.277255-4.435221-0.688520$ H $-2.630000-4.981070-1.565999$ $\begin{array}{llll}\text { C }-2.877561 & -4.630535 & 0.554591\end{array}$ $\begin{array}{llll}\mathrm{H} & -3.692387 & -5.349805 & 0.666708\end{array}$ C $-2.447074-3.8964951 .65980$ H $-2.934535 \quad-4.035814 \quad 2.625756$ C $-1.399436 \quad-2.977710 \quad 1.544534$ $\begin{array}{llll}\text { C }-0.905147 & -2.171840 & 2.734443\end{array}$ $\begin{array}{llll}\mathrm{H} & -0.442846 & -1.248127 & 2.348124\end{array}$ C $0.191274 \quad-2.919702 \quad 3.508611$ H $1.035304 \quad-3.182475 \quad 2.856002$ H $-0.210432-3.850879 \quad 3.943346$ н $0.585654 \quad-2.282743 \quad 4.314755$ C $-2.027865-1.744986 \quad 3.684264$ H $-2.841745-1.241408 \quad 3.140326$ $\mathrm{H}-2.841745-1.241408 \quad 3.140326$ $\mathrm{H}-1.626170-1.0438614 .431508$ C $-2.453363-2.6050614 .226405$ $\begin{array}{llll}\text { C } 3.109503 & 1.648785 & 1.288385\end{array}$ $\begin{array}{llll}\text { B } 2.594207 & 0.960498 & 2.693281 \\ \text { F } 3.125342 & -0.365150 & 2.831172\end{array}$ F $1.165198 \quad 0.869198 \quad 2.694533$ F 3.0206851 .7490393 .792182 C $3.9320972 .801478 \quad 1.316102$ H $4.2185693 .193676 \quad 2.295895$ C $4.357173 \quad 3.428110 \quad 0.146462$ H $4.993698 \quad 4.316767 \quad 0.189223$ C $3.9504882 .924708-1.109864$ H $4.2729653 .432245 \quad-2.022771$ C $3.134304 \quad 1.793602-1.193989$ C $2.7003661 .237600-2.540951$ C $2.7003661 .237600-2.540951$ $\begin{array}{llll}\text { H } & 1.824137 & 0.591523 & -2.370992\end{array}$ $\begin{array}{llll}\text { C } 2.277364 & 2.333361 & -3.527192\end{array}$ . $1.8375031 .874429-4.426160$ $1.5315903 .008379-3.081627$ C $3.1388032 .940113-3.849116$ $\begin{array}{llll}\text { C } 3.794582 & 0.359551 & -3.169101\end{array}$ H $3.446140-0.056639-4.127583$ H $4.7059890 .953140-3.351505$ H $4.062059-0.481924-2.514687$ C $-0.093262 \quad 1.948772-0.262523$ H $0.5205412 .198320-1.138555$ H $0.357566 \quad 2.338458 \quad 0.663675$ H $-1.1217672 .308921-0.395413$ $\begin{array}{llll}\mathrm{N} & -1.760598 & -0.029815 & 0.042059\end{array}$ $\begin{array}{llll}\text { C }-2.919357 & -0.143048 & 0.102399\end{array}$ $\begin{array}{llll}C & -4.317709 & -0.257360 & 0.194149\end{array}$ H $-4.8699510 .674902 \quad 0.337511$ C -4.8699510 .6749020 .337511$ $\begin{array}{lllll}C & -4.955419 & -1.449564 & 0.11344\end{array}$ $\begin{array}{llll}\mathrm{H}-6.043586 & -1.492137 & 0.194356 \\ \mathrm{H}-4.398880 & -2.379954 & -0.032277\end{array}$ 75 $22 \mathrm{R}=\mathrm{n}-\mathrm{Pr} \mathrm{ET} / \mathrm{pi}$

$\mathrm{Ni}-0.044095-0.063188-0.008275$ $\begin{array}{llll}\mathrm{N} & 1.937012 & -0.100512 & 0.110696\end{array}$ N $0.344244 \quad 1.890051-0.107145$ $\begin{array}{llll}\text { C } 1.594929 & 2.204017 & 0.032958\end{array}$ C $2.517349 \quad 1.060385 \quad 0.002809$ C $-0.205741-1.962422 \quad 0.410230$ $\begin{array}{llll}\text { C }-1.551611 & -2.658759 & 0.419583\end{array}$ C $-1.428706-4.073703 \quad 1.008760$ н $0.519175-2.481400-0.236665$ H $0.190355-1.885856 \quad 1.437211$ H $-1.957834-2.744195-0.602340$ $\begin{array}{lll}\mathrm{H} & -1.957834-2.744195 & -0.602340\end{array}$ $\begin{array}{llll}\mathrm{H} & -2.275518 & -2.085118 & 1.022019\end{array}$ H $-1.074091-4.0318642 .050209$ $\begin{array}{llll}-1.074091-4.031864 & 2.050209\end{array}$ $\begin{array}{llll}\text { C }-1.756369 & 0.035524 & -1.072787\end{array}$ 
C $-0.685643-0.391848-1.871569$ H $-0.550083-1.439106-2.148723$ H $-0.112556 \quad 0.301745-2.493123$ H $-2.501265-0.673087-0.710049$ H $-2.076086 \quad 1.081414-1.064555$ C $2.745728-1.2876820 .077492$ $\begin{array}{llll}\text { C }-0.689800 & 2.855852 & 0.096725\end{array}$ C $2.107353 \quad 3.581304 \quad 0.290158$ C $3.9839031 .288727-0.144912$ C $4.1854471 .612840-1.17912$ $\begin{array}{llll}\mathrm{H} & 4.185447 & 1.612840 & -1.179901\end{array}$ $\begin{array}{lllll}\mathrm{H} & 4.330084 & 2.073290 & 0.544693\end{array}$ $\begin{array}{lllll}\mathrm{H} & 4.550347 & 0.365718 & 0.029225\end{array}$ $\begin{array}{llllll}\text { H } 2.602659 & 3.601174 & 1.274989\end{array}$ $\begin{array}{llll}\text { H } & 1.288286 & 4.310419 & 0.306571 \\ \text { H } & 2.849034 & 3.869346 & -0.471035\end{array}$ $\begin{array}{lllr}\text { H } & 2.849034 & 3.869346 & -0.471035 \\ \text { C } & -1.278201 & 2.845043 & 1.375569\end{array}$ $\begin{array}{llll}\text { C } & -1.278201 & 2.845043 & 1.375569 \\ \text { C } & -1.129366 & 3.681091 & -0.951041\end{array}$ $\begin{array}{llll}\text { C } & -1.129366 & 3.681091 & -0.951041 \\ \text { C }-2.326644 & 3.742862 & 1.593436\end{array}$ $\begin{array}{llll}\text { C }-2.326644 & 3.742862 & 1.593436 \\ \text { C }-2.180283 & 4.563968 & -0.670849\end{array}$ $\begin{array}{llll}\text { C }-2.772840 & 4.602538 & 0.587818\end{array}$ B -0.7505531 .8330262 .538085$ F $-1.593004 \quad 1.857117 \quad 3.662595$ $\begin{array}{llll}F & -0.758747 & 0.443438 & 2.012270\end{array}$ F $0.589745 \quad 2.117913 \quad 2.904169$ C $-0.523372 \quad 3.621617 \quad-2.343184$ C $0.131830 \quad 4.955206 \quad-2.746206$ C $-1.561278 \quad 3.202294-3.395757$ $\begin{array}{llll}C & -1.561278 & 3.202294 & -3.395757\end{array}$ $\begin{array}{llll}\mathrm{H} & 0.264717 & 2.853504 & -2.335717\end{array}$ H $-0.622233 \quad 5.756670-2.816582$ H $-0.6222335 .756670-2.816582$ $\begin{array}{lllll}\text { H } & 0.619468 & 4.856621 & -3.729764\end{array}$ H $-2.368280 \quad 3.949175 \quad-3.471546$ $\begin{array}{lllll}\mathrm{H} & -2.016117 & 2.233454 & -3.143925\end{array}$ H $-1.084552 \quad 3.111362-4.38468$ $\begin{array}{llll}\mathrm{H} & -2.798365 & 3.752622 & 2.577960\end{array}$ $\mathrm{H}-3.591946 \quad 5.2980460 .780520$ H $-2.5444285 .227437-1.457854$ C $3.184171-1.790089-1.162403$ C $3.014886-1.9112431 .311621$ C $3.925292-2.980896-1.107994$ C $3.759963-3.0962451 .296798$ $\begin{array}{lll}\text { C } 4.212658 & -3.636023 & 0.096079\end{array}$ C $2.572534-1.2965362 .630082$ $\begin{array}{llll}\text { C } 2.036407 & -2.334589 & 3.629564\end{array}$ $\begin{array}{llll}\text { C } & 2.036407 & -2.334589 & 3.629564 \\ \text { C } 3.710812 & -0.487578 & 3.272970\end{array}$ $\begin{array}{llll}\text { C } 3.710812 & -0.487578 & 3.272970 \\ \text { H } 1.755422 & -0.589273 & 2.422694\end{array}$ $\begin{array}{llll}\text { H } 1.755422 & -0.589273 & 2.422694 \\ \text { H } 1.272008 & -2.975303 & 3.165140\end{array}$ $\begin{array}{llll}\text { H } & 1.272008 & -2.975303 & 3.165140 \\ \text { H } & 2.842209 & -2.984051 & 4.010012\end{array}$ $\begin{array}{llll}\mathrm{H} & 2.842209 & -2.984051 & 4.010012 \\ \mathrm{H} & 1.583374 & -1.820053 & 4.491630\end{array}$ $\begin{array}{llll}\mathrm{H} & 1.583374 & -1.820053 & 4.491630 \\ \mathrm{H} & 4.071828 & 0.300680 & 2.596347\end{array}$ $\begin{array}{llll}\text { H } & 4.071828 & 0.300680 & 2.596347\end{array}$ $\begin{array}{llll}\text { H } & 4.563245 & -1.145450 & 3.512171 \\ \text { H } & 3.361440 & -0.007162 & 4.200618\end{array}$ H $3.361440-0.0071624 .200618$ B $2.876572-1.174335-2.664600$ F $3.986359-1.466362-3.506895$ F $1.711733-1.806188-3.193327$ F $2.6686890 .243531-2.654689$ H $3.985914-3.600472 \quad 2.239512$ H $4.791811-4.5645420 .095218$ $\begin{array}{llll}\text { H } & 4.291933 & -3.392112 & -2.052688\end{array}$ 76

$22 \mathrm{n}-\mathrm{Pr} \mathrm{AC} / \mathrm{pi}$

Ni $0.0174890 .032991-0.037842$ $\mathrm{N} 2.0062950 .016801-0.042229$ N $0.364037-1.959432-0.034606$ C $1.616807-2.286880-0.050962$ C $2.550700-1.1578100 .091658$ $\begin{array}{llll}\text { C } & 0.015004 & 1.977493 & -0.298629\end{array}$ C $-1.257970 \quad 2.781946-0.353890$ $\begin{array}{llll}\text { C }-0.946590 & 4.218444 & -0.815683\end{array}$ H $0.713407 \quad 2.356932 \quad 0.462838$ H $0.502718 \quad 1.940678 \quad-1.287390$ H $-1.739220 \quad 2.838533 \quad 0.634116$ H $-1.9854192 .331701-1.047423$ H $-0.511661 \quad 4.221253 \quad-1.826862$ H $-0.225782 \quad 4.699506 \quad-0.136877$ $\mathrm{H}-1.867156 \quad 4.823077-0.834451$ $\begin{array}{llll}\mathrm{H}-1.867156 & 4.823077 & -0.834451\end{array}$ $\begin{array}{llll}C & -0.609522 & 0.239436 & 1.823276\end{array}$

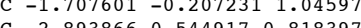
C -2.8938660 .5449170 .818397$ $\mathrm{H}-1.906429-1.280012 \quad 0.952763$ $\mathrm{H}-1.906429-1.2800120 .952763$ H $0.000163-0.4757172 .380862$ H $-0.561490 \quad 1.260350 \quad 2.203666$ $\begin{array}{llll}\text { C } 2.863533 & 1.172827 & -0.039432\end{array}$ $\begin{array}{llll}\text { C }-0.661744 & -2.888474 & -0.393688\end{array}$ $\begin{array}{llll}\text { C } 2.138171 & -3.659878 & -0.313540\end{array}$ $\begin{array}{llll}\text { C } 3.996610 & -1.411854 & 0.355060\end{array}$ H $4.105197-1.663843 \quad 1.424865$ H $4.601021-0.5157460 .168046$ H $4.373924-2.250391-0.246958$ H $2.787855-3.636389-1.203343$ H $2.741421-4.0169080 .536168$ $\begin{array}{llll}\text { H } 2.741421 & -4.016908 & 0.536168\end{array}$ $\begin{array}{llll}\text { H } & 1.317129 & -4.360297 & -0.506708\end{array}$ $\begin{array}{llll}C & -1.169198 & -2.725840 & -1.697641\end{array}$ C $-1.177053-3.8175130 .526172$ $\begin{array}{llll}\text { C }-2.233142 & -4.628501 & 0.088580\end{array}$
C $-2.750453-4.509558-1.196867$ B $-0.540074-1.623191-2.714859$ F $-1.269402-1.536922-3.907099$ F $-0.598747-0.279485-2.066546$ F $0.830690-1.879324-2.973693$ C $-0.650432 \quad-3.950499 \quad 1.944186$ $\begin{array}{llll}\text { C }-0.059413 & -5.368768 & 2.209710\end{array}$ C $-1.726909-3.630954 \quad 2.991305$ H $0.166349-3.225077 \quad 2.076577$ H $0.719345-5.6238521 .474364$ $\begin{array}{llll}\text { H } & 0.719345 & -5.623852 & 1.474364\end{array}$ $\begin{array}{llll}\mathrm{H} & 0.381486 & -5.410783 & 3.219821\end{array}$ H $-0.850968-6.134316 \quad 2.146390$ $\mathrm{H}-2.129333-2.6158512 .860104$ $\mathrm{H}-1.304776-3.7036864 .006034$ H $-2.567367-4.339412 \quad 2.918859$ H $-2.627559-3.453688-3.082535$ H $-3.577358-5.149428-1.509432$ $\begin{array}{llll}\mathrm{H} & -2.661049 & -5.361550 & 0.774592\end{array}$ C $3.2264821 .685372-1.302342$ C 3.2692731 .7404191 .180227 C $4.0473142 .816003-1.329719$ $\begin{array}{llll}\text { C } 4.092119 & 2.875454 & 1.079157\end{array}$ C $4.4779553 .412626-0.146666$ C $2.7979251 .010696-2.595776$ $\begin{array}{lllll}\text { C } 2.281780 & 1.995361 & -3.647283\end{array}$ C $3.9394100 .176378-3.197230$

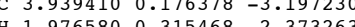

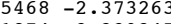
$\begin{array}{llll}\mathrm{H} & 1.491073 & 2.641874 & -3.239247\end{array}$ H $1.8622951 .437923-4.498810$ H $3.0883612 .641997-4.029420$ H $4.319518-0.566310-2.476962$ H $3.582598-0.365164-4.089658$ H $4.7846910 .825853-3.488251$ B $2.823278 \quad 1.255086 \quad 2.691072$ F 3.8412801 .6173993 .611674 F $2.608492-0.161427 \quad 2.767869$ F 1.6102791 .9221553 .045425 H $4.350374 \quad 3.233799 \quad-2.292618$ H $5.118575 \quad 4.298871-0.185301$ H $4.435515 \quad 3.334627 \quad 2.010270$ 76

$\begin{array}{llll}22 \mathrm{n}-\mathrm{Pr} & \mathrm{AC} / \mathrm{N} & & \\ \mathrm{Ni} & -0.077336 & -0.465350 & -0.291365\end{array}$ $\mathrm{N} 1.168674 \quad 1.024549-0.010450$ $\mathrm{N}-1.168674 \quad 1.024549-0.010450$ $\begin{array}{lllll}\mathrm{N} & -1.361035 & 0.934035 & -0.005373\end{array}$ $\begin{array}{llll}\text { C } 0.612141 & 2.186065 & 0.132890\end{array}$ $\begin{array}{lllll}\text { C } & 0.612141 & 2.186065 & 0.132890 \\ \text { C } & -3.585429 & 2.544070 & -2.669835\end{array}$ C $-3.5854292 .544070-2.669835$ $\begin{array}{llll}\text { C } 2.595651 & 0.884370 & -0.011913\end{array}$ $\begin{array}{llll}\text { C }-2.770542 & 0.737219 & 0.222243\end{array}$ $\begin{array}{lllll}\text { C }-1.643952 & 3.343527 & 0.513461\end{array}$ C $1.327197 \quad 3.481453 \quad 0.289652$ H $0.955886 \quad 4.019597 \quad 1.175273$ H $2.409078 \quad 3.323056 \quad 0.372097$ H $1.153170 \quad 4.099788 \quad-0.605747$ H $-1.327572 \quad 4.182529-0.124812$ $\mathrm{H}-1.451004 \quad 3.606795 \quad 1.566945$ H $-2.721325 \quad 3.171709 \quad 0.408568$ $\begin{array}{llll}C & -3.158239 & 0.462072 & 1.546958\end{array}$ $\begin{array}{llll}C & -3.662207 & 0.785350 & -0.86121\end{array}$ $\begin{array}{llll}C & -4.525489 & 0.244461 & 1.755907\end{array}$ $\begin{array}{llll}C & -5.016793 & 0.565052 & -0.584522\end{array}$ $\begin{array}{llll}C & -5.448203 & 0.296812 & 0.711184\end{array}$ F $-2.771586-0.006766 \quad 3.981305$ F $-1.071736-0.589494 \quad 2.502362$ F $-1.455321 \quad 1.643648 \quad 3.000604$ C $-3.205736 \quad 1.106955-2.275164$ C $-3.7586530 .119675-3.31180$ H $-2.1071351 .034189-2.301443$ $\mathrm{H}-3.161518 \quad 3.280606-1.972362$ H $-3.2128492 .775678-3.680427$ H $-4.681372 \quad 2.666531-2.665762$ H $-3.533293-0.919782-3.032894$ H $-3.310573 \quad 0.320430 \quad-4.297522$ H $-4.8515810 .215186-3.412507$ $\begin{array}{llll}\mathrm{H} & -4.853444 & 0.023811 & 2.773497\end{array}$ $\begin{array}{llll}\mathrm{H}-4.553444 & 0.023811 & 2.773497 \\ \mathrm{H}-6.508943 & 0.122161 & 0.903135\end{array}$ $\begin{array}{llll}H & -5.742437 & 0.600394 & -1.398811\end{array}$ $\begin{array}{llll}\text { C } 3.206169 & 0.507838 & 1.198656\end{array}$ C $3.2917461 .064612-1.219113$ $\begin{array}{llll}C & 4.594594 & 0.339003 & 1.190082\end{array}$ $\begin{array}{llll}\text { C } 4.681841 & 0.881193 & -1.162388\end{array}$ $\begin{array}{llll}\text { C } 5.336470 & 0.529356 & 0.018464\end{array}$ $\begin{array}{llll}\text { C } 2.411375 & 0.332284 & 2.482613\end{array}$ $\begin{array}{lll}\text { C } 2.621423 & -1.049390 & 3.113398\end{array}$ C $2.733438 \quad 1.445547 \quad 3.491868$ H $1.340025 \quad 0.406403 \quad 2.245923$ H $2.379051-1.8456832 .394129$ H $3.662344-1.186873 \quad 3.449497$ H $1.956863-1.163463 \quad 3.983480$ \begin{tabular}{l}
$1.556863 \quad-1.163463 \quad 3.983480$ \\
\hline
\end{tabular}

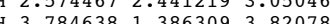
3.7846381 .3863093 .820782 B $2.08567371 .472280-2.635979$ B $2.5567371 .472280-2.635979$ F $2.0065712 .792882-2.531712$

F $1.501566 \quad 0.559581 \quad-2.923244$ $\begin{array}{lllll}\text { H } & 5.104664 & 0.062651 & 2.116516\end{array}$ H $6.423934 \quad 0.402839 \quad 0.037107$ H $5.246047 \quad 1.024428-2.088889$ C $-1.471492-1.793540-0.629826$ $\begin{array}{llll}\text { C }-1.150434 & -3.260355 & -0.857961\end{array}$ C $-2.431633-4.085683-1.044886$ н $-2.092931-1.6654320 .271936$ н $-1.991795-1.363862-1.503277$ $\mathrm{H}-0.512118-3.385714-1.748355$ $\begin{array}{llll}\mathrm{H} & -0.512118 & -3.385714 & -1.748355 \\ \mathrm{H} & -0.591956 & -3.659620 & 0.005153\end{array}$ H $-0.591956-3.6596200 .005153$ $\begin{array}{lllll}\mathrm{H} & -3.095025 & -3.976981 & -0.173073\end{array}$ H $-2.989711-3.746472-1.931767$ H $-2.202854-5.156444-1.173991$ $\mathrm{N} 1.185530-1.667067-0.612118$ $\begin{array}{llll}\text { C } 2.066216 & -2.383192 & -0.875953\end{array}$ C $3.084756-3.288926-1.219606$ C $4.388922-2.949102-1.217263$ H $2.757043-4.293897-1.499247$ H $5.145580-3.684275-1.498706$ $\mathrm{H}$ 22 R=CHCNEt ET/pi

Ni $-0.059719 \quad-0.036240 \quad 0.119878$ $\begin{array}{llll}\mathrm{N} & 1.948761 & -0.129586 & 0.216684\end{array}$ $\begin{array}{llll}\mathrm{N} & 0.387049 & 1.892175 & -0.004618\end{array}$ C 1.6453372 .1797620 .107213

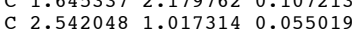
C $-0.337507 \quad-1.990446 \quad 0.465931$ C $-0.534971-2.199414 \quad 1.975702$ $\begin{array}{llll}\text { C }-0.533037 & -3.684137 & 2.361274\end{array}$ $\begin{array}{lllll}\text { H } & -0.533037 & -3.684137 & 2.361274\end{array}$ $\begin{array}{llll}\text { H } & 0.620678 & -2.412255 & 0.114546 \\ \text { C } & -1.393625 & -2.642084 & -0.262323\end{array}$ $\begin{array}{llll}\text { C } & -1.393625 & -2.642084 & -0.262323 \\ \text { H } & -1.479543 & -1.730024 & 2.290357\end{array}$ $\begin{array}{lllll}\mathrm{H} & -1.479543 & -1.730024 & 2.29035 \\ \mathrm{H} & 0.263688 & -1.672697 & 2.512150\end{array}$ н $-1.377698-4.212227 \quad 1.891957$ H $0.396169 \quad-4.176594 \quad 2.032723$ H $-0.620740 \quad-3.793380 \quad 3.453893$ C $-1.8064730 .159372-0.894426$ C $-0.762161-0.192155-1.760725$ H $-0.627584-1.211565-2.126580$ H $-0.2040870 .555875-2.327810$ $\mathrm{H}-2.536030-0.588693-0.578279$ H $-2.536030-0.588693-0.578279$ $\begin{array}{llll}\mathrm{H} & -2.121592 & 1.200314 & -0.780889\end{array}$ $\begin{array}{llll}\text { C } 2.750257 & -1.321638 & 0.111955\end{array}$ C 2.1931363 .5489850 .326976 $\begin{array}{llll}C & 2.193136 & 3.548985 & 0.326976\end{array}$ $\begin{array}{llll}\text { C } 3.992133 & 1.220368 & -0.231100\end{array}$ $\begin{array}{llll}\text { H } 4.090942 & 1.492217 & -1.296460\end{array}$ H $4.4080072 .034981 \quad 0.379753$ H $4.568748 \quad 0.301482 \quad-0.070938$ H $2.771506 \quad 3.554826 \quad 1.265388$ H $1.387120 \quad 4.286976 \quad 0.415936$ H $2.871699 \quad 3.833123-0.492639$ C $-1.246874 \quad 2.985043 \quad 1.374025$ $\begin{array}{llll}\text { C }-0.976445 & 3.697201 & -0.983943\end{array}$ C $-2.277235 \quad 3.919467 \quad 1.503600$ $\begin{array}{llll}\text { C }-2.015277 & 4.617352 & -0.792252\end{array}$ $\begin{array}{llll}\text { C }-2.662323 & 4.730908 & 0.434726\end{array}$ B -0.7666572 .0318962 .601463$ F $-1.646395 \quad 2.106037 \quad 3.692920$ F $-0.756992 \quad 0.625530 \quad 2.130315$ $\begin{array}{llll}\text { F } 0.561570 & 2.334900 & 2.995729\end{array}$ C $-0.301080 \quad 3.574344-2.339231$ $\begin{array}{llll}\text { C } 0.429002 & 4.879312 & -2.736475\end{array}$ $\begin{array}{lllll}C & -1.293371 & 3.171155 & -3.439607\end{array}$ $\begin{array}{llll}\text { H } & 0.458099 & 2.780841 & -2.274772 \\ \text { H } & 1.150093 & 5.189581 & -1.963501\end{array}$ H $-0.291661 \quad 5.703071-2.876666$ H $0.973903 \quad 4.733283-3.684444$ H $-2.047573 \quad 3.958796-3.598011$ н $-1.8207842 .241666-3.181864$ н $-0.7598513 .011534-4.389809$ H $-2.784937 \quad 3.995988 \quad 2.467022$ $\mathrm{H}-3.474305 \quad 5.450784 \quad 0.553423$ $\begin{array}{lllll}\text { H } & -2.326312 & 5.249661 & -1.625845\end{array}$ $\begin{array}{llll}C & 3.050110 & -1.823744 & -1.16838\end{array}$ $\begin{array}{llll}\text { C } 3.182578 & -1.925325 & 1.310474\end{array}$ C $3.787605-3.018622-1.195022$ $\begin{array}{llll}\text { C } 3.919258 & -3.115287 & 1.210465\end{array}$ $\begin{array}{llll}C & 4.208351 & -3.667017 & -0.033567\end{array}$ $\begin{array}{llll}\text { C } 2.950957 & -1.275443 & 2.666672\end{array}$ $\begin{array}{llll}\text { C } 2.715742 & -2.291222 & 3.799447\end{array}$ $\begin{array}{llll}\text { C } 4.124269 & -0.357919 & 3.052281\end{array}$ H $2.056916-0.6374362 .590882$ H $1.953140-3.0352283 .527850$ H $3.645234-2.828420 \quad 4.051818$ H $2.382038-1.762524 \quad 4.706797$ H $4.267056 \quad 0.449683 \quad 2.322119$ H $5.060382-0.938445 \quad 3.104397$ H $3.938734 \quad 0.102767 \quad 4.035804$ B $2.629877-1.186988-2.636677$ B $2.629877-1.186988-2.636677$ F $3.702859-1.421151-3.540536$ 
F $2.381702 \quad 0.226713-2.583726$ $\begin{array}{rlll}\mathrm{N} & -2.277817 & -3.179269 & -0.809775\end{array}$

$22 \mathrm{R}=\mathrm{CHCNEt} \mathrm{AC} / \mathrm{pi}$

Ni $0.007249 \quad 0.003572 \quad-0.006804$ $\begin{array}{llll}\text { N } 2.007837 & 0.023517 & -0.085576\end{array}$ N $0.393210-1.974007-0.064009$ C $1.649753-2.282787-0.093170$ $\begin{array}{llll}\text { C } 2.570393 & -1.141936 & 0.047156\end{array}$ C $-0.1237892 .017931-0.099402$ C $-0.1237892 .017931-0.099402$ C $-0.4850272 .420872-1.531681$ $\begin{array}{llll}C & -0.289063 & 3.927495 & -1.756374\end{array}$ $\begin{array}{llll}C & -0.951365 & 2.696879 & 0.860991\end{array}$ N -1.6137643 .2920371 .619441$ H 0.9176462 .2769500 .151822 H $-1.5275292 .137272-1.746080$ H $0.149436 \quad 1.860098 \quad-2.228904$ H $0.7501114 .222735-1.539986$ H $-0.953060 \quad 4.512841-1.10227$ H $-0.5160714 .184411-2.802932$ C $-0.740176-0.027926 \quad 1.840615$ $\begin{array}{llll}\text { C } & -1.776843 & -0.319651 & 0.920833\end{array}$ $\begin{array}{llll}C & -2.880888 & 0.515780 & 0.581112\end{array}$ $\mathrm{N}-3.849598 \quad 1.083981 \quad 0.256910$ H $-2.008757-1.367186 \quad 0.696296$ H $-0.219267 \quad-0.853954 \quad 2.329074$ $\begin{array}{llll}\mathrm{H} & -0.658632 & 0.931521 & 2.350638\end{array}$ $\begin{array}{llll}\mathrm{H} & -0.658632 & 0.931521 & 2.350638 \\ \mathrm{C} & 2.863223 & 1.182132 & -0.107379\end{array}$ C $-0.618864-2.931927-0.397299$ $\begin{array}{llll}\text { C } & -0.618864 & -2.931927 & -0.397299 \\ \text { C } 2.185146 & -3.647023 & -0.369000\end{array}$ $\begin{array}{llll}\text { C } & 2.185146 & -3.647023 & -0.369000 \\ \text { C } 4.016178 & -1.372374 & 0.327550\end{array}$ н $4.112829-1.4879701 .422809$ H $4.626168-0.506040 \quad 0.042797$ H $4.392025-2.277775-0.16634$ H $2.867501-3.599826-1.232615$ $\begin{array}{lllll}\text { H } & 2.758189 & -4.020565 & 0.494786\end{array}$ H $1.374353-4.345339-0.607558$ C $-1.156132-2.793292-1.690682$ $\begin{array}{llll}\text { C }-1.081628 & -3.875204 & 0.536637\end{array}$ $\begin{array}{llll}\text { C } & -2.201531 & -3.650757 & -2.038948\end{array}$ $\begin{array}{llll}\text { C }-2.131467 & -4.709284 & 0.129952\end{array}$ C $-2.693251-4.599640-1.141467$ C $-2.693251-4.599640-1.141467$ B $-0.552977-1.698092-2.728551$ F $-1.315598-1.607456-3.898207$ $\begin{array}{llll}\text { F } & -0.581730 & -0.361801 & -2.073710\end{array}$ F $0.808270-1.965943-3.024325$ C $-0.502880-4.0087881 .935170$ $\begin{array}{llll}\text { C } 0.111180 & -5.405650 & 2.165447\end{array}$ C $-1.547885-3.704032 \quad 3.01820$ H $0.306910-3.271190 \quad 2.041832$ H $0.859606-5.653690 \quad 1.396007$ H $0.598067-5.450347 \quad 3.154746$ H $-0.670354 \quad-6.1844202 .135879$ н $-1.977468 \quad-2.6995692 .892912$ H $-1.088316-3.760429 \quad 4.017524$ H $-2.374220-4.431534 \quad 2.980241$ $\begin{array}{llll}\mathrm{H} & -2.635239 & -3.553494 & -3.035859\end{array}$ H $-3.520602-5.253192-1.426556$ $\begin{array}{llll}\text { H } & -2.522928 & -5.452002 & 0.827014\end{array}$ C $3.2427321 .659979-1.381475$ C $3.261237 \quad 1.770196 \quad 1.104450$ $\begin{array}{llll}\text { C } 4.062927 & 2.789560 & -1.423361\end{array}$ $\begin{array}{llll}\text { C } 4.084726 & 2.904583 & 0.987216\end{array}$ $\begin{array}{llll}\text { C } 4.480045 & 3.412848 & -0.247170\end{array}$ $\begin{array}{llll}\text { C } 2.838533 & 0.940237 & -2.660307\end{array}$ $\begin{array}{llll}\text { C } 2.553475 & 1.886808 & -3.830095\end{array}$ C $3.903934-0.081096-3.09367$ H $1.917658 \quad 0.372356-2.461066$ H $1.8616692 .692879-3.546001$ H $2.102496 \quad 1.318003-4.657835$ H $3.479767 \quad 2.348081-4.210358$ H $4.095180-0.829580-2.311373$ H $3.564945-0.617068-3.995015$ H $4.8582710 .428445-3.314795$ B $2.7976591 .288285 \quad 2.614131$ F $3.719821 \quad 1.8001573 .561303$ F $2.758973 \quad-0.143994 \quad 2.728938$ $\begin{array}{llll}\text { F } & 2.758973 & -0.143994 & 2.728938 \\ \text { F } & 1.497535 & 1.799811 & 2.881013\end{array}$ H $4.378411 \quad 3.187691 \quad-2.389954$ $\begin{array}{lllll}\text { H } & 4.378411 & 3.187691 & -2.389954 \\ \text { H } & 5.117732 & 4.300391 & -0.300819\end{array}$ $\begin{array}{llll}\mathrm{H} & 5.117732 & 4.300391 & -0.300819 \\ \mathrm{H} & 4.416293 & 3.384401 & 1.912090\end{array}$ 77 $22 \mathrm{R}=\mathrm{CHCNE}$ t $\mathrm{AC} / \mathrm{N}$ Ni $0.020671-0.689714 \quad-0.393057$ N $1.217761 \quad 0.836789-0.106973$ $\begin{array}{lllll}\mathrm{N} & -1.280597 & 0.702307 & -0.517191\end{array}$ C $-0.804461 \quad 1.914576-0.441768$ $\begin{array}{llll}\text { C } & 0.655453 & 1.993772 & -0.257295\end{array}$ $\begin{array}{lllll}C & -3.030883 & 1.946204 & -3.63184\end{array}$ $\begin{array}{llll}\text { C } 2.605501 & 0.733393 & 0.264091\end{array}$ $\begin{array}{llll}\text { C }-2.713566 & 0.525465 & -0.467447\end{array}$ C $-1.623224 \quad 3.156888-0.409683$ C $1.351335 \quad 3.305861-0.193675$ H $0.816738 \quad 4.0023690 .468665$ 0.3167389
4.00236920 .1485994 $1.3843043 .731775-1.210560$ H $-1.209578 \quad 3.908464-1.098783$
H $-1.5886393 .561164 \quad 0.616511$ $\begin{array}{llll}\mathrm{H} & -2.673494 & 2.957895 & -0.65056\end{array}$ C $-3.277871 \quad 0.4254790 .817988$ C $-3.452703 \quad 0.432146 \quad-1.658958$ $\begin{array}{lllll}\text { C } & -4.664611 & 0.242213 & 0.871998\end{array}$ $\begin{array}{llll}C & -4.836186 & 0.256378 & -1.534488\end{array}$ C $-5.440073 \quad 0.163513-0.284029$ $\begin{array}{llll}\text { B }-2.387367 & 0.494864 & 2.195071\end{array}$ F $-3.217430 \quad 0.287409 \quad 3.321104$ F $-1.368384-0.5214252 .162380$ F $-1.731187 \quad 1.764228 \quad 2.310381$ F -1.7311871 .7642282 .310381$ $\begin{array}{llll}C & -2.808629 & 0.547348 & -3.030997\end{array}$ C $-3.305353-0.522562-4.011167$ $\begin{array}{llll}\mathrm{H} & -1.723464 & 0.402728 & -2.907211\end{array}$ H $-2.6284382 .739925-2.986961$ $\mathrm{H}-2.5374922 .021305-4.613779$ H $-4.1087572 .135493-3.769030$ $\mathrm{H}-3.167601-1.535227-3.608112$ H $-2.735099-0.455270-4.950399$ H $-4.370275-0.378039-4.254244$ H $-5.127222 \quad 0.158185 \quad 1.857171$ H $-6.520340 \quad 0.020499-0.214408$ н $-5.447312 \quad 0.182851 \quad-2.435017$ $\begin{array}{llll}\text { C } 2.872655 & 0.577931 & 1.639900\end{array}$ $\begin{array}{lllll}C & 3.601683 & 0.740584 & -0.727971\end{array}$ $\begin{array}{llll}C & 4.2073636 & 0.7405841 & 0.727971\end{array}$ C $4.9231260 .588920-0.27$ $\begin{array}{llll}C & 4.923126 & 0.588920 & -0.272346\end{array}$ $\begin{array}{llll}C & 5.235869 & 0.430894 & 1.076003\end{array}$ $\begin{array}{lll}c & 1.778764 & 0.5868572 .694581\end{array}$ $\begin{array}{llll}\text { C } 1.741946 & -0.721382 & 3.488564\end{array}$ C 1.9188711 .7789503 .651587 H $0.8019730 .680314 \quad 2.202524$ H $1.634599-1.5849372 .815526$ H $2.662235-0.855553 \quad 4.081190$ H $0.880076-0.715707 \quad 4.172652$ H $1.947085 \quad 2.733667 \quad 3.100137$ H $2.846366 \quad 1.705874 \quad 4.247135$ H $1.059441 \quad 1.808139 \quad 4.341854$ $\begin{array}{llll}\text { B } 3.339694 & 0.858217 & -2.350539\end{array}$ F $4.5653721 .180338-2.995762$ F $2.3651261 .854516-2.650291$ F $2.885689-0.409105-2.841704$ $\begin{array}{llll}\mathrm{H} & 4.446106 & 0.317248 & 3.084439\end{array}$

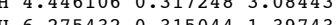
$\begin{array}{llll}\text { H } & 6.275432 & 0.315044 & 1.397438 \\ \text { H } & 5.717797 & 0.608736 & -1.023778\end{array}$ C $-1.255083-2.176929-0.676734$ $\begin{array}{llll}C & -1.255083 & -2.176929 & -0.676734 \\ C & -1.113895 & -3.256460 & 0.406946\end{array}$ C $-1.113895-3.2564600 .406946$ $\begin{array}{llll}C & -2.258518 & -4.274889 & 0.346954 \\ H & -2.246247 & -1.702369 & -0.596278\end{array}$ $\begin{array}{llll}\text { H } & -2.246247 & -1.702369 & -0.596278 \\ \text { C }-1.087958 & -2.688473 & -2.008481\end{array}$ $\begin{array}{llll}C & -1.087958 & -2.688473 & -2.008481\end{array}$ $\begin{array}{lllll}\mathrm{H} & -0.149357 & -3.773444 & 0.289880\end{array}$ H $-1.117363-2.749577 \quad 1.383374$ H $-3.228279-3.7785810 .506506$ H $-2.286576-4.778041-0.632576$ H $-2.135569-5.042489 \quad 1.127289$ $\mathrm{N} 1.401952-1.834220-0.507789$ C $2.428150-2.322545-0.755118$ C $3.685189-2.836739-1.129387$ $\begin{array}{llll}C & 4.281823 & -3.856242 & -0.489082\end{array}$ H 4.126682 $\begin{array}{llll}\text { H } 5.261414 & -4.210598 & -0.81401 \\ \text { H } 3.817918 & -4.344464 & 0.370778\end{array}$ $\mathrm{N}-0.961304-3.129680-3.086205$

63

$23 \mathrm{R}=\mathrm{Me}$

$\begin{array}{llll}\mathrm{Ni} & -0.134517 & 0.011848 & -0.009822\end{array}$ $\begin{array}{llll}1.710668 & 0.067875 & -0.048616\end{array}$ $\begin{array}{llll}2.442405 & 1.307482 & 0.044344\end{array}$ $\begin{array}{llll}2.3142 & -1.09967 & -0.082244\end{array}$ $\begin{array}{llll}3.829532 & -1.276842 & -0.04626\end{array}$ $\begin{array}{llll}4.483412 & -0.306829 & 0.600738\end{array}$ $4.343999-1.336379-1.315558$ $\begin{array}{llll}4.136003 & -2.455188 & 0.566281\end{array}$ $1.403331-2.242143-0.067719$ $\begin{array}{lll}1.799098 & -3.641481-0.539278\end{array}$ $\begin{array}{lll}1.79 & 75332 & -3.535425-1.507591\end{array}$ $\begin{array}{lll}2.75332 & -3.535425 & -1.507591 \\ 2.300428 & -4.448028 & 0.434886\end{array}$ $\begin{array}{lll}2.300428 & -4.448028 & 0.434886\end{array}$ $\begin{array}{lll}0.167903 & -1.90057 & 0.197684\end{array}$ $\begin{array}{llll}0.167903 & -1.90057 & 0.197684 \\ -0.869534 & -2.817834 & 0.501443\end{array}$ $\begin{array}{llll}-0.869534 & -2.817834 & 0.501443 \\ -2.054774 & -2.706523 & -0.251552\end{array}$ $-0.746081-3.6922971 .608839$ $\begin{array}{lll}-3.072427 & -3.623932 & 0.009781\end{array}$ $\begin{array}{llll}-1.804679 & -4.579176 & 1.822994\end{array}$ $\begin{array}{lll}-2.938043 & -4.580629 & 1.013256\end{array}$ $-2.272572-1.490431-1.281316$ $-1.356069-1.413897-2.330723$ $-2.001034-0.226937-0.396389$ $\begin{array}{llll}0.353534 & -3.557111 & 2.650348\end{array}$ $0.747142-4.8904353 .294363$ $-0.081576-2.5362483 .717232$ $\begin{array}{lll}-0.081576 & -2.536248 & 3.717232 \\ 1.257732 & -3.146151 & 2.1837\end{array}$ $\begin{array}{lll}1.2577077 & -3.651856 & 2.536276\end{array}$ $\begin{array}{lll}0.984077 & -5.651856 & 2.536276 \\ 1.633572 & -4.742016 & 3.929192\end{array}$ H $-0.05577-5.2787113 .940405$
$-0.249071-1.5436443 .274593$ $-1.009938-2.8699714 .210066$ $\begin{array}{lll}0.709856 & -2.427046 & 4.474458\end{array}$ $-3.990471-3.559119-0.575958$ $\begin{array}{llll}-3.735521 & -5.301859 & 1.199663\end{array}$ $\begin{array}{llll}-1.747939 & -5.274808 & 2.659832\end{array}$ $\begin{array}{llll}2.92407 & 1.905578 & -1.130459\end{array}$ $\begin{array}{llll}2.525459 & 1.874526 & 1.32563\end{array}$ $\begin{array}{lll}3.533735 & 3.157053 & -0.986316\end{array}$ $\begin{array}{llll}3.159362 & 3.118769 & 1.405138\end{array}$ $\begin{array}{lll}3.655087 & 3.764632 & 0.269516\end{array}$ $\begin{array}{llll}2.764649 & 1.282211 & -2.506909\end{array}$ $\begin{array}{lll}2.764649 & 1.282211 & -2.506909 \\ 4.099533 & 1.289272 & -3.308541\end{array}$ $\begin{array}{lll}4.099533 & 1.289272 & -3.308541 \\ 1.654289 & 1.963076 & -3.320755\end{array}$ $\begin{array}{lll}1.654289 & 1.963076 & -3.320755 \\ 2.473788 & 0.2295 & -2.381641\end{array}$ $\begin{array}{lll}2.473788 & 0.2295 & -2.381641 \\ 4.379275 & 2.315471 & -3.599041\end{array}$ $\begin{array}{lll}4.379275 & 2.315471 & -3.59904 \\ 3.979505 & 0.697089 & -4.23111\end{array}$ $\begin{array}{llll}4.919311 & 0.858302 & -2.71339\end{array}$ $\begin{array}{lll}0.680248 & 1.87257 & -2.821309\end{array}$ $\begin{array}{lll}1.572245 & 1.49344 & -4.31420\end{array}$ $\begin{array}{lll}1.875688 & 3.03391 & -3.458546\end{array}$ $\begin{array}{lll}1.950342 & 1.111641 & 2.666248 \\ 0.531062 & 0.891098 & 2.53631\end{array}$ $\begin{array}{lll}0.531062 & 0.891098 & 2.53631\end{array}$ $\begin{array}{llll}2.193358 & 1.907006 & 3.810264\end{array}$ $\begin{array}{llll}2.577742 & -0.162542 & 2.806501\end{array}$ $\begin{array}{lll}3.920615 & 3.668591 & -1.87125\end{array}$ $\begin{array}{lll}8.742615 & 0.351334\end{array}$ $\begin{array}{lll}3.252594 & 3.578237 & 2.39121 \\ -0.45884 & 1.889402 & -0.287019\end{array}$ $\begin{array}{lll}-0.45884 & 1.889402 & -0.287019 \\ -0.727251 & 2.144401 & 0.754118\end{array}$ $\begin{array}{lll}-1.313795 & 1.980874 & -0.97045\end{array}$ $\mathrm{H}$ $23 \mathrm{R}=\mathrm{Me} \mathrm{ET/pi}$

Ni $0.057027 \quad-0.020510 \quad 0.075682$ N $2.022970-0.038788 \quad 0.015415$ C $2.752918 \quad 1.203696-0.031839$ $\begin{array}{llll}\text { C } 2.581950 & -1.189920 & 0.270762\end{array}$ C $4.078239-1.4086390 .533103$ F $4.829409-0.3109170 .366340$ F $4.564043-2.346907-0.341735$ F $4.288075-1.876494 \quad 1.784161$ $\begin{array}{llll}\text { C } 1.628467 & -2.315401 & 0.329776\end{array}$ $\begin{array}{llll}\text { C } 2.077274 & -3.775349 & 0.164920\end{array}$ F $2.636547-3.960839-1.051729$ F $3.000235-4.1061591 .113300$ $\begin{array}{llll}F & 1.067447 & -4.658436 & 0.282370\end{array}$ N $0.388270-1.9484620 .421331$ C $-0.756761-2.8127290 .377856$ $\begin{array}{llll}\text { C } & -1.406124 & -2.894509 & -0.866348\end{array}$ $\begin{array}{llll}\text { C }-1.260298 & -3.381747 & 1.558836\end{array}$ $\begin{array}{llll}\text { C }-2.576918 & -3.654965 & -0.914124\end{array}$ $\begin{array}{llll}\text { C }-2.438497 & -4.128884 & 1.445068\end{array}$ $\begin{array}{llll}\text { C }-3.088528 & -4.278097 & 0.223928\end{array}$ $\begin{array}{llll}\text { B }-0.849321 & -2.092247 & -2.162487\end{array}$ F $0.463915-2.461608-2.510050$ F $-1.721256-2.183408-3.253620$ F $-0.783584-0.636926-1.781636$ C $-0.591708-3.209647 \quad 2.912816$ C $-0.101731-4.5501323 .981809$ $\begin{array}{lll}C & -0.101731-4.550132 & 3.481809\end{array}$ $\begin{array}{llll}C & -1.523312 & -2.517730 & 3.920797\end{array}$ H $0.288977-2.5615392 .784174$ H $-0.952081-5.223015 \quad 3.677904$ H $0.429436-4.384876 \quad 4.432163$ $\begin{array}{llll}\text { H } & 0.429436 & -4.384876 & 4.432163 \\ \text { H } & -2.407085 & -3.141829 & 4.130430\end{array}$ $\begin{array}{llll}\mathrm{H} & -2.407085 & -3.141829 & 4.130430 \\ \mathrm{H}-1.874915 & -1.545798 & 3.546845\end{array}$ $\begin{array}{llll}\mathrm{H} & -1.874915 & -1.545798 & 3.546845 \\ \mathrm{H} & -0.991965 & -2.348036 & 4.870269\end{array}$ H $-3.092612-3.739409-1.872379$ H $-4.003782-4.8695260 .165191$ $\begin{array}{llll}\text { H } & -2.854495 & -4.599732 & 2.337279\end{array}$ C $2.9905091 .737015-1.316249$ C $3.069158 \quad 1.8649301 .165198$ $\begin{array}{lll}\text { C } 3.605186 & 2.985435 & -1.386572\end{array}$ C $3.684674 \quad 3.1202901 .023192$ C $3.955948 \quad 3.685396-0.219265$ C $2.6543960 .961888-2.579340$ $\begin{array}{llll}\text { C } 2.654396 & 0.961888 & -2.579340 \\ \text { C } 2.103329 & 1.849143 & -3.706462\end{array}$ $\begin{array}{llll}C & 3.873645 & 0.172459 & -3.083634\end{array}$ $\begin{array}{llll}\text { H } 1.872091 & 0.226495 & -2.332637\end{array}$ H $1.2796372 .487247-3.352823$ H $2.8870752 .501755-4.124770$ H $1.7253221 .214962-4.523307$ H $4.254850-0.520332-2.320810$ H $4.6912600 .863597 \quad-3.347325$ $\begin{array}{llll}\text { H } 3.603906 & -0.415442 & -3.974950\end{array}$ B $2.758414 \quad 1.379545 \quad 2.710683$ F $3.9202971 .588585 \quad 3.495263$ F $1.6946632 .184638 \quad 3.227072$ F $2.371942 \quad 0.003818 \quad 2.786195$ H $3.814765 \quad 3.427591-2.362401$ H $4.434150 \quad 4.666471-0.294469$ $\begin{array}{lllll}\text { H } & 3.952261 & 3.654454 & 1.938274\end{array}$ C $-0.133350 \quad 1.809980-0.551192$ $\begin{array}{llll}\text { C } & -0.133350 & 1.809980 & -0.55119 \\ \mathrm{H} & 0.136121 & 1.676877 & -1.607932\end{array}$ H $0.5708812 .454354 \quad-0.011276$ H $-1.168106 \quad 2.163040-0.454968$ 
$\begin{array}{llll}\text { C } & -0.575991 & 0.590076 & 1.899086\end{array}$ C -1.6673330 .1594691 .139858$ H $-0.345067 \quad 1.647196 \quad 2.041171$ H $-0.055883 \quad-0.0697892 .597337$ H $-2.336720 \quad 0.879947 \quad 0.665325$ 70 $23 \mathrm{R}=\mathrm{Me} \mathrm{AC} / \mathrm{pi}$

$\mathrm{Ni} \quad 0.091383 \quad-0.030864 \quad 0.013645$

$\begin{array}{llll}\mathrm{N} & 2.020495 & -0.05127 & 0.022048\end{array}$

N $\quad 0.375853-2.007133-0.218972$

$\begin{array}{llll}\text { C } & 1.629711 & -2.341786 & -0.255815\end{array}$

$\begin{array}{llll}2.552401 & -1.248485 & 0.055702\end{array}$

$\begin{array}{lll}-0.08128 & 1.907402 & -0.041873\end{array}$

$\begin{array}{lll}0.10286 & 2.325941 & 0.955547\end{array}$

$\begin{array}{lll}0.702618 & 2.200022 & -0.752277\end{array}$

$\begin{array}{lll}-1.09298 & 2.090194 & -0.42836\end{array}$

$\begin{array}{lll}-0.471488 & -0.15249 & 1.940305\end{array}$

$\begin{array}{llll}-1.636094 & -0.210604 & 1.147633\end{array}$

$\begin{array}{lll}-2.590081 & 0.846617 & 1.13329\end{array}$

$\begin{array}{lll}-3.411834 & 1.675954 & 1.140249\end{array}$

$\begin{array}{lll}-2.057009 & -1.17217 & 0.832418 \\ 0.009632 & -1.071773 & 2.273574\end{array}$

$\begin{array}{lll}0.009632 & -1.071773 & 2.273574 \\ -0.190522 & 0.748813 & 2.489038\end{array}$

$\begin{array}{lll}-0.190522 & 0.748813 & 2.489038 \\ 2.834791 & 1.150403 & 0.042376\end{array}$

$\begin{array}{lll}2.834791 & 1.150403 & 0.042376 \\ -0.750374 & -2.816225 & -0.570939\end{array}$

$2.126058-3.682466-0.82832$

$\begin{array}{lll}2.126058 & -3.682466 & -0.82832 \\ 4.013221 & -1.471442 & 0.472443\end{array}$

$\begin{array}{lll}4.013221 & -1.471442 & 0.472443 \\ 4.895232 & -0.801822 & -0.345513\end{array}$

$\begin{array}{lll}4.895232 & -0.801822 & -0.345513 \\ 4.183593 & -1.073937 & 1.73002\end{array}$

$\begin{array}{lll}4.183593 & -1.073937 & 1.73002 \\ 4.347969 & -2.778351 & 0.498151\end{array}$

$\begin{array}{lll}4.347969 & -2.778351 & 0.498151 \\ 3.372536 & -3.541553 & -1.361317\end{array}$

$\begin{array}{lll}3.372536 & -3.541553 & -1.361317 \\ 1.305211 & -4.11091 & -1.798625\end{array}$

$\begin{array}{llll}2.177717 & -4.673333 & 0.104849\end{array}$

$-1.406976-2.434025-1.759413$

$\begin{array}{llll}-1.259259 & -3.778838 & 0.321442\end{array}$

$-2.595421-3.094006-2.065312$

$-2.465533-4.393626-0.04003$

$-3.127967-4.066488-1.216516$

$-0.810951-1.265462-2.715534$

$-1.64546-0.998162-3.804598$

$-0.764006-0.011672-1.894891$

$0.515237-1.522956-3.121723$

$\begin{array}{llll}0.515237 & -1.522956 & -3.121723 \\ -0.618792 & -4.136947 & 1.649667\end{array}$

$\begin{array}{lll}-0.618792 & -4.136947 & 1.649667 \\ -0.325353 & -5.639549 & 1.747106\end{array}$

$\begin{array}{lll}-1.491234 & -3.698869 & 2.835955 \\ 0.341574 & -3.602785 & 1.733082\end{array}$

$\begin{array}{lll}0.341574 & -3.602785 & 1.733082\end{array}$

$\begin{array}{llll}0.237383 & -5.991647 & 0.873341\end{array}$

$\begin{array}{llll}0.260148 & -5.856482 & 2.654558\end{array}$

$\begin{array}{llll}-1.260699 & -6.218162 & 1.805064\end{array}$

$\begin{array}{llll}-1.716991 & -2.624087 & 2.813448\end{array}$

$-0.978777-3.9206833 .782981$

$-2.448484-4.2428712 .832105$

$\begin{array}{lll}-3.117407 & -2.812366-2.983257\end{array}$

$-4.069795-4.558975-1.462484$

$\begin{array}{llll}-2.897346 & -5.139247 & 0.630055\end{array}$

$\begin{array}{llll}3.208993 & 1.627234 & -1.235793\end{array}$

$\begin{array}{lll}3.096519 & 1.840925 & 1.238527\end{array}$

$\begin{array}{lll}3.937548 & 2.81857 & -1.282915\end{array}$

$\begin{array}{lll}3.937548 & 2.81857 & -1.282915 \\ 3.842806 & 3.023164 & 1.107283\end{array}$

$\begin{array}{lll}3.842806 & 3.023164 & 1.107283 \\ 4.265228 & 3.513116 & -0.122814\end{array}$

$\begin{array}{lll}4.265228 & 3.513116 & -0.122814 \\ 2.847683 & 0.940461 & -2.546935\end{array}$

$\begin{array}{lll}2.847683 & 0.940461 & -2.546935 \\ 1.853772 & 1.767061 & -3.372117\end{array}$

$\begin{array}{lll}1.853772 & 1.767061 & -3.372117 \\ 4.083809 & 0.640992 & -3.409691\end{array}$

$\begin{array}{lll}4.083809 & 0.640992-3.409691 \\ 2.356374 & -0.018018-2.334739\end{array}$

$\begin{array}{lll}2.356374 & -0.018018 & -2.334739 \\ 0.909952 & 1.93052 & -2.833917\end{array}$

$\begin{array}{lll}0.909952 & 1.93052 & -2.833917 \\ 1.611005 & 1.229692 & -4.301159\end{array}$

$\begin{array}{lll}1.611005 & 1.229692 & -4.30115 \\ 2.280305 & 2.748042 & -3.63711\end{array}$

$\begin{array}{llll}4.843414 & 0.071128 & -2.853678\end{array}$

$\begin{array}{llll}3.781081 & 0.054907 & -4.293187\end{array}$

$\begin{array}{llll}4.552744 & 1.570601 & -3.773171\end{array}$

$\begin{array}{lll}2.464215 & 1.54752 & 2.739111\end{array}$

$\begin{array}{lll}1.192153 & 2.184636 & 2.791351\end{array}$

$\begin{array}{lll}3.194674 & 2.193319 & 3.752838\end{array}$

$\begin{array}{lll}2.294347 & 0.164268 & 3.016723\end{array}$

$\begin{array}{lll}2.294347 & 0.164268 & 3.016723 \\ 4.245877 & 3.207307 & -2.256511\end{array}$

$\begin{array}{lll}4.245877 & 3.207307 & -2.256511 \\ 4.833185 & 4.44603 & -0.188682\end{array}$

$\begin{array}{lll}4.068191 & 3.574387 & 2.024311\end{array}$

$3 \mathrm{R}=\mathrm{Me} \quad \mathrm{AC} / \mathrm{N}$

Ni $0.101860 \quad 0.094548 \quad 0.005939$ $\begin{array}{lllll}\mathrm{N} & 1.965117 & 0.032913 & -0.343217\end{array}$ $\begin{array}{llll}\text { C } 2.755341 & 1.230839 & -0.237919\end{array}$ $\begin{array}{llll}\text { C } 2.497001 & -1.166159 & -0.355747\end{array}$ C $4.008160-1.414034-0.277492$ F $4.551183-1.492495-1.525913$ $\begin{array}{llll}\text { F } 4.259489 & -2.589868 & 0.347521\end{array}$ $\begin{array}{lllll}\text { F } 4.675832 & -0.465076 & 0.394641\end{array}$ $\begin{array}{llll}\text { C } 1.514426 & -2.245612 & -0.408355\end{array}$ $\begin{array}{llll}\text { C } 1.883818 & -3.693144 & -0.735040\end{array}$ $\begin{array}{lllll}\text { F } 2.290179 & -4.333745 & 0.408721\end{array}$ F $0.878312-4.416888-1.240843$ F $2.912972-3.740487-1.620458$ $\begin{array}{llll}\text { F } & 2.912972 & -3.740487 & -1.620458 \\ \text { N } 0.306035 & -1.842202 & -0.122669\end{array}$ $\begin{array}{llll}\mathrm{N} & 0.306035 & -1.842202 & -0.122669 \\ \mathrm{C} & -0.858807 & -2.682132 & -0.214585\end{array}$ $\begin{array}{llll}\text { C } & -0.858807 & -2.682132 & -0.214585 \\ \text { C } & -1.497155 & -2.786420 & -1.464697\end{array}$ C $-1.362955-3.2241780 .977901$
C $-2.675055-3.544350-1.481957$ $\begin{array}{llll}C & -2.546542 & -3.966455 & 0.890563\end{array}$ $\begin{array}{llll}\text { C }-3.195502 & -4.135744 & -0.329541\end{array}$ в $-0.970988-2.041492-2.840763$ F $-1.672192-2.573469-3.953631$ F $-1.237919-0.640297-2.738875$ F $0.434353-2.227836-3.017123$ C $-0.669328-3.028477 \quad 2.315658$ $\begin{array}{llll}\text { C }-0.240933 & -4.368046 & 2.931381\end{array}$ C -1.543429 C $-1.543429-2.2313113 .294280$ H $0.242075-2.4352202 .146448$ $0.333629-4.1935283 .854636$ H $0.333629-4.1935283 .854636$ $\mathrm{H}-1.119328-4.983310-3.186139$ H $-1.809572-1.2496062 .876055$ H $-0.996530-2.059546 \quad 4.234407$ H $-2.472618-2.776859 \quad 3.527905$ H $-3.177787-3.666500-2.443604$ H $-4.108872-4.732893-0.380029$ н $-2.958257 \quad-4.420686 \quad 1.793555$ C $3.3163461 .781966-1.401605$ C $2.804631 \quad 1.830298 \quad 1.030986$ $\begin{array}{llll}\text { C } 3.990578 & 2.999544 & -1.247038\end{array}$ C $3.5029623 .037058 \quad 1.122965$ C $4.095438 \quad 3.623978 \quad 0.002072$ C $3.1722381 .140686-2.773810$ $\begin{array}{llll}C & 3.172238 & 1.140686 & -2.773810\end{array}$ $\begin{array}{llll}C & 2.006811 & 1.753474 & -3.566104\end{array}$ $\begin{array}{llll}\text { C } 4.470516 & 1.205384 & -3.590081 \\ \text { H } 2.9288002 & 0.077473 & -2.639164\end{array}$ $\begin{array}{llll}\text { H } 2.928802 & 0.077473 & -2.639164\end{array}$ $1.0465681 .578862-3.063283$ H $2.1513262 .839253-3.693499$ H $1.9464761 .284825-4.560910$ H $5.326205 \quad 0.825175-3.011891$ H $4.6960802 .236191-3.908274$ H $4.3634320 .594246 \quad-4.499944$ B 2.1001011 .1451962 .336165 F $0.662170 \quad 0.936621 \quad 2.050536$ F $2.187144 \quad 1.988574 \quad 3.457757$ F $2.645148-0.130765 \quad 2.608257$ H $4.4481613 .469603-2.120458$ H $4.635781 \quad 4.571004 \quad 0.089902$ H 3.5688453 .5167292 .101856 C $-0.179729,1.950651-0.585866$ C -0.179729 .51672920 .101856$ $\begin{array}{llll}\mathrm{H} & 0.800854 & 2.374915 & -0.826647\end{array}$ H $-0.852613 \quad 1.897491-1.450230$ $\mathrm{H}-0.6337862 .4521270 .279644$ $\mathrm{N}-1.6945080 .0345910 .164272$ $\begin{array}{lllll}c-2.837206 & -0.174999 & 0.208517\end{array}$ $\begin{array}{llll}C & -4.189947 & 0.562117 & 0.183819\end{array}$ $\begin{array}{llll}\text { C }-5.215098 & 0.224695 & 0.588560\end{array}$ H $-4.355147-1.572782-0.203078$ н $\quad-5.045119 \quad 1.232087 \quad 0.974198$ $\mathrm{H}$
75

$23 \mathrm{R}=\mathrm{n}-\mathrm{Pr} \mathrm{ET} / \mathrm{pi}$

Ni $0.058627-0.0118920 .100976$ N $2.040400-0.0218950 .219171$ N $0.424758 \quad 1.939396-0.037909$ $\begin{array}{llll}\text { C } 1.667427 & 2.280299 & 0.105956\end{array}$ C $2.608326 \quad 1.1460780 .083222$ C $-0.150429-1.902987 \quad 0.590940$ $\begin{array}{llll}C & -0.150429 & -1.902987 & 0.590940 \\ C & -1.543464 & -2.475388 & 0.751537\end{array}$ $\begin{array}{llll}C & -1.543464 & -2.475388 & 0.751537\end{array}$ H $0.483668-2.488218-0.091535$ $\begin{array}{llll}\text { H } & 0.483668 & -2.488218 & -0.091535 \\ \text { H } 0.334002 & -1.793514 & 1.572346\end{array}$ $\begin{array}{llll}\mathrm{H} & 0.334002 & -1.793514 & 1.572346 \\ \mathrm{H} & -1.994067 & -2.695648 & -0.230090\end{array}$ $\begin{array}{llll}\mathrm{H} & -1.994067 & -2.695648 & -0.230090 \\ \mathrm{H} & -2.191871 & -1.751215 & 1.271354\end{array}$ $\mathrm{H}-2.191871-1.7512151 .271354$ H $-0.852249-4.524099 \quad 1.104036$ H $-1.135796 \quad-3.5818112 .589205$ H $-2.520361-4.2054701 .658670$ C $-1.578785-0.011963-1.111785$ C $-0.445426-0.423249-1.816078$ н $-0.258060-1.473437-2.047554$ H $0.163825 \quad 0.269969 \quad-2.401680$ н $-2.321651-0.733753-0.774877$ H $-1.925143 \quad 1.024406-1.142485$ C $2.765234-1.2642710 .101595$ $\begin{array}{llrl}\text { C } & -0.706905 & 2.815797 & 0.061963\end{array}$ $\begin{array}{lllll}\text { C } 2.104697 & 3.735393 & 0.328327\end{array}$

$\begin{array}{llll}\text { C } 4.118142 & 1.377298 & -0.060185\end{array}$ $\begin{array}{llll}\text { C } 4.118142 & 1.377298 & -0.060185 \\ \text { F } 4.357790 & 2.441414 & -0.870532\end{array}$ $\begin{array}{llll}\text { F } & 4.357790 & 2.441414 & -0.870532\end{array}$ F $4.8019820 .336373-0.542358$ F $3.1766823 .808432 \quad 1.152704$ $\begin{array}{lllll}\mathrm{F} & 1.134120 & 4.488480 & 0.876385\end{array}$ F $2.448070 \quad 4.318241-0.857412$ C $-1.403813 \quad 2.747587 \quad 1.283393$ C $-1.158802 \quad 3.545290-1.049919$ $\begin{array}{lllll}\text { C }-2.576191 & 3.497340 & 1.383894\end{array}$ $\begin{array}{llll}\text { C }-2.348872 & 4.267477 & -0.887388\end{array}$ $\begin{array}{lllll}\text { C }-3.050553 & 4.254976 & 0.312387\end{array}$ B $-0.882604 \quad 1.810972 \quad 2.500648$ F 0.4306362 .1162452 .905953 F 0.4306362 .1162452 .905953 F -0.8374590 .3982061 .975205$ 10.8374590 .3982061 .975205 C $-0.056034 \quad 4.994798-2.803402$
C $-1.2907292 .914771-3.498784$ H $0.4832432 .978036-2.302345$ H $0.516199 \quad 5.498538-2.011955$ $\begin{array}{lllll}\text { H } & -0.955567 & 5.596582 & -3.01234\end{array}$ H $0.555936 \quad 4.969251 \quad-3.719151$ H $-2.228266 \quad 3.474143-3.650571$ $\mathrm{H}-1.550180 \quad 1.874571-3.258852$ H $-0.7324402 .916268-4.448122$ H $-3.124003 \quad 3.4656502 .327410$ H $-3.974187 \quad 4.828408 \quad 0.407380$ H $-2.7320394 .847923-1.728040$ C $3.077375-1.743556-1.185775$ $\begin{array}{llll}\text { C } & 3.077375 & -1.743556 & -1.185775 \\ \text { C } 3.99831 & -1.991980 & 1.285662\end{array}$ C $3.640777-3.025559-1.234578$ C $3.580962-3.2578121 .164152$ C $3.889362-3.785326-0.086225$ $\begin{array}{lll}\text { C } 2.707433 & -1.421480 & 2.663365\end{array}$ $\begin{array}{lll}\text { C } 2.054656 & -2.457482 & 3.626891\end{array}$ C $3.981592-0.8661293 .318492$ H $1.999506-0.584907 \quad 2.550629$ H $1.203446-2.969975 \quad 3.154188$ H $2.786324-3.221667 \quad 3.938630$ H $1.696915-1.940899 \quad 4.532396$ H $4.453829-0.095323 \quad 2.696339$ H $4.713862-1.676403 \quad 3.469194$ H $3.742536-0.4227514 .298180$ B $2.842536-0.4227514 .298180$ B $2.886210-0.990290-2.644084$ F $4.093695-1.124616-3.373759$ H $1.8347261-1.634411-3.363678$ H $3.787391-3.8390042 .065388$ H $4.335010-4.781030-0.168038$ $\begin{array}{llll}\text { H } & 3.904903 & -3.423743 & -2.217649 \\ \text { F } & 2.569002 & 0.403303 & -2.510360\end{array}$ 76

$23 \mathrm{R}=\mathrm{n}-\mathrm{Pr} \mathrm{AC} / \mathrm{pi}$

$\mathrm{Ni} \quad 0.219188 \quad 0.033346 \quad-0.178580$ $\begin{array}{llll}\mathrm{N} 2.168054 & -0.173562 & 0.095967\end{array}$ N $0.342951-1.974655-0.070243$ C $1.535493-2.429775-0.003458$ $\begin{array}{llll}\text { C } 2.563967 & -1.401758 & 0.290266\end{array}$ $\begin{array}{llll}\text { C } & 0.445834 & 1.933682 & -0.662454\end{array}$ C $-0.742815 \quad 2.762858-1.112084$ C $-0.204546 \quad 4.012401-1.845394$ H 0.9891612 .3681720 .172546 $\begin{array}{llllllllll}\mathrm{H} & 0.989161 & 2.368172 & 0.172546\end{array}$ $1.3408943 .095557-0.247627$ $\mathrm{H}-1.3408943 .095557-0.247627$ H $-1.3887232 .197720-1.787681$ $\begin{array}{llll}\text { H } & 0.345175 & 3.728147 & -2.754707\end{array}$ $\begin{array}{lllll}\mathrm{H} & 0.471977 & 4.587440 & -1.207514 \\ \mathrm{H} & -1.0449420 & 4.660760 & -2.141001\end{array}$ $\begin{array}{llll}\text { H } & -1.049420 & 4.660760 & -2.141001 \\ \text { C }-0.644696 & 0.514026 & 1.604205\end{array}$ $\begin{array}{llll}\text { C }-0.644696 & 0.514026 & 1.604205 \\ \text { C }-1.646367 & 0.076645 & 0.745820\end{array}$ $\begin{array}{llll}\text { C } & -1.646367 & 0.076645 & 0.745820 \\ \text { C } & -2.706378 & 0.923106 & 0.275083\end{array}$ N $-3.635392 \quad 1.556287 \quad-0.056055$ н $-1.948647 \quad-0.973061 \quad 0.711095$ н $-0.124488 \quad-0.205135 \quad 2.267437$ H $-0.498626 \quad 1.552311 \quad 1.876105$ $\begin{array}{llll}C & 3.125652 & 0.917958 \quad 0.048843\end{array}$ $\begin{array}{llll}\text { C } & -0.825459 & -2.752606 & -0.334779 \\ \text { C } & 1.937857 & -3.857677 & -0.430040\end{array}$ $\begin{array}{llll}\text { C } 3.935079 & -1.796860 & 0.883938\end{array}$ F $4.495009-0.7788411 .482150$ F $4.886571-2.227698-0.017621$ F $3.776688-2.8210051 .755291$ F $0.899290-4.527426-1.009515$ F $2.968197-3.782500-1.406234$ $\begin{array}{llll}\text { F } 2.432242 & -4.615397 & 0.633815\end{array}$ $\begin{array}{llll}\text { C } & -1.443519 & -2.554537 & -1.585738\end{array}$ $\begin{array}{llll}\text { C }-1.391524 & -3.553415 & 0.681448\end{array}$ $\begin{array}{llll}\text { C }-2.632111 & -3.217285 & -1.830016\end{array}$ $\begin{array}{llll}\text { C }-2.592976 & -4.203227 & 0.395223\end{array}$ $\begin{array}{llll}C & -3.218846 & -4.052821 & -0.851916\end{array}$ B $-0.746047-1.606077-2.716046$ F $-0.531117-0.240203-2.108861$ F $0.543652-2.071085-3.072527$ F $-1.571455-1.423829-3.827661$ $\begin{array}{llll}F & -1.571455 & -1.423829 & -3.827661\end{array}$ C $-0.805364-3.6528592 .088250$ C $-0.578569-5.1442382 .521001$ c $-1.686757-2.936579 .3 .109983$ H $0.181176-3.1717462 .097394$ H $0.051245-5.6722561 .797323$ H $-0.088438-5.166764 \quad 3.510451$ H $-1.541272-5.669505 \quad 2.604780$ H $-1.824953-1.8736422 .864199$ H $-1.236568-2.992863 \quad 4.11624$ H $-2.687393-3.396503 \quad 3.152902$ $\begin{array}{llll}\mathrm{H} & -3.125599 & -3.082634 & -2.786118\end{array}$ H $-4.150800-4.565837-1.051665$ H $-3.064405 \quad-4.822779 \quad 1.147702$ C $3.7635611 .115316-1.203548$ C $3.324400 \quad 1.764469 \quad 1.147061$ $\begin{array}{llll}\text { C } 4.683355 & 2.170301 & -1.299029\end{array}$ C $4.6833552 .170301-1.299029$ C $4.276195 \quad 2.7852490 .985155$ $\begin{array}{llll}\text { C } 4.945619 & 2.980028 & -0.207789\end{array}$ $\begin{array}{llll}\text { C } 3.463444 & 0.273671 & -2.441318\end{array}$

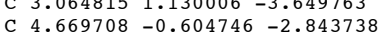


H $2.624685-0.388654-2.236000$ H $2.224107 \quad 1.790487-3.407058$ H $2.7574320 .473739-4.473053$ H $3.894379 \quad 1.755443-3.996392$ H $4.989450-1.251408-2.016043$ H $4.395077-1.241868-3.695765$ H $5.5256700 .022804 \quad-3.123883$ B $2.496694 \quad 1.7134452 .575514$ F 1.2984842 .4433362 .401156 F 3.1575622 .4048483 .595788 $\begin{array}{llll}F & 3.157562 & 2.404848 & 3.59578\end{array}$ $\begin{array}{llll}\text { F } 2.207133 & 0.358440 & 2.937822\end{array}$ H $5.1978242 .333255-2.250060$ $\begin{array}{llll}\mathrm{H} & 5.684673 & 3.797481 & -0.30145 \\ \mathrm{H} & 4.475803 & 3.424278 & 1.849657\end{array}$ 76

$23 \mathrm{R}=\mathrm{n}-\mathrm{Pr} \mathrm{AC} / \mathrm{N}$

Ni $-0.003224 \quad 0.072068 \quad-0.133107$ $\begin{array}{llll}\mathrm{N} & 1.890735 & 0.011084 & -0.112720\end{array}$ N $0.231414 \quad-1.881361-0.287868$ $\begin{array}{llll}\text { C } 1.465830 & -2.264751-0.401585\end{array}$ $\begin{array}{llll}\text { C } 2.433121 & -1.181883 & -0.188926\end{array}$ $\begin{array}{llll}\text { C }-0.094660 & 2.048419 & -0.064571\end{array}$ C $-1.397017 \quad 2.697489-0.480104$ C $-1.194056 \quad 4.209373-0.677230$ $\begin{array}{llll}\text { H } & 0.717332 & 2.313076 & -0.756940\end{array}$ H 0.1952282 .2468690 .978080 . 0.1952282 .246869 $\begin{array}{llll}\mathrm{H} & -2.172178 & 2.543432 & 0.286382\end{array}$ H $-1.77015922 .254660-1.418365$ H $-0.465730 \quad 4.405362-1.47885$ $\begin{array}{llllll}\mathrm{H} & -0.809873 & 4.674161 & 0.243894\end{array}$ $\mathrm{H}-2.143270 \quad 4.700080-0.946376$ $\begin{array}{lllll}\mathrm{N} & -1.638908 & 0.022621 & 0.651247\end{array}$ $\begin{array}{llll}C & -2.720762 & -0.104354 & 1.06199\end{array}$ $\begin{array}{llll}\text { C }-4.031093 & -0.221042 & 1.56974\end{array}$ $\begin{array}{llll}\text { C }-4.882944 & -1.173702 & 1.157714\end{array}$ H $-4.317621 \quad 0.5181432 .322589$ H $-5.887901-1.226911 \quad 1.575045$ C $-0.601105 \quad-5.244220 \quad 2.054130$ C $2.703203 \quad 1.199507 \quad 0.005408$ $\begin{array}{lllll}\text { C }-0.938227 & -2.660878 & -0.555857\end{array}$ $\begin{array}{llll}\text { C } 1.863822 & -3.690352 & -0.795545\end{array}$ C $3.934287-1.445011-0.059268$ F $4.514373-1.519643-1.298603$ $\begin{array}{llll}\text { F } & 4.514373 & -1.519643 & -1.298603 \\ \text { F } & 4.603064 & -0.520858 & 0.635506\end{array}$ $\begin{array}{llll}\text { F } & 4.603064 & -0.520858 & 0.635506 \\ \text { F } & 4.139482 & -2.642468 & 0.557498\end{array}$ $\begin{array}{llll}\text { F } & 4.139482 & -2.642468 & 0.557498 \\ \text { F } & 2.153406 & -4.435576 & 0.312646\end{array}$ $\begin{array}{llll}\text { F } 2.153406 & -4.435576 & 0.312646 \\ \text { F } 2.958693 & -3.688986 & -1.594660\end{array}$ $\begin{array}{llll}\text { F } & 2.958693 & -3.688986 & -1.594660 \\ \text { F } & 0.887645 & -4.334266 & -1.460945\end{array}$

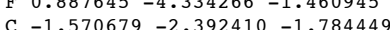
$\begin{array}{llll}\text { C } & -1.570679 & -2.392410 & -1.784449 \\ \text { C } & -1.467550 & -3.514809 & 0.424366\end{array}$ $\begin{array}{llll}\text { C } & -1.467550 & -3.514809 & 0.424366 \\ \text { C }-2.762936 & -3.074600 & -2.036740\end{array}$ $\begin{array}{llll}\text { C }-2.674646 & -4.156650 & 0.116653\end{array}$ C $-3.315153-3.951839-1.101772$ B $-0.937760-1.349536-2.865888$ F $-1.763882-1.223178-3.995477$ F $-0.856433-0.014468-2.224705$ F $0.379642-1.711240-3.22995$ C $-0.798707-3.748662 \quad 1.768575$ C $-1.579355-3.079743-2.909844$ $\begin{array}{lllll}C & -1.579355 & -3.079743 & 2.909844\end{array}$ $\begin{array}{llll}\text { H } 0.195685 & -3.279768 & 1.748906\end{array}$ $\begin{array}{llll}\mathrm{H}-0.079204 & -5.742392 & 1.224113\end{array}$ $\mathrm{H}-0.006414-5.3752962 .971989$ $\mathrm{H}-1.568401-5.7503902004865$ H $-1.621816-1.990164 \quad 2.773174$ H $-1.084923-3.2771253 .874125$ $\begin{array}{lllll}\mathrm{H} & -2.609074 & -3.470445 & 2.960218\end{array}$ H $-3.255834-2.899592-2.995056$ H $-4.244398-4.480992-1.322917$ H $-3.111842-4.8391810 .84742$ C $3.1929231 .773353-1.182561$ $\begin{array}{llll}\text { C } 2.863612 & 1.759821 & 1.284279\end{array}$ $\begin{array}{llll}\text { C } 3.874265 & 2.993621 & -1.059585\end{array}$ C $3.572593 \quad 2.966198 \quad 1.332352$ C 4.0616613 .5889330 .183356 C $2.987350 \quad 1.161705-2.562236$ C $1.909391 \quad 1.901932-3.363637$ $\begin{array}{llll}\text { C } & 4.287537 & 1.117958 & -3.377469\end{array}$ $\begin{array}{llll}\text { C } & 4.287537 & 1.117958 & -3.377469 \\ \text { H } & 2.639298 & 0.125366 & -2.447226\end{array}$ $\begin{array}{lllll}\text { H } & 2.639298 & 0.125366 & -2.447226 \\ \text { H } & 0.923505 & 1.827944 & -2.885014\end{array}$ $\begin{array}{lllll}\text { H } & 0.923505 & 1.827944 & -2.885014 \\ \text { H } & 2.173508 & 2.966655 & -3.476318\end{array}$ $\begin{array}{lllll}\text { H } & 2.173508 & 2.966655 & -3.476318 \\ \text { H } & 1.816829 & 1.454452 & -4.365756\end{array}$ $\begin{array}{llll}\mathrm{H} & 1.816829 & 1.454452 & -4.365756 \\ \mathrm{H} & 5.104781 & 0.646655 & -2.807968\end{array}$ $\begin{array}{lllll}\text { H } & 5.104781 & 0.646655 & -2.807968 \\ \text { H } & 4.611971 & 2.131521 & -3.669887\end{array}$ $\begin{array}{lllll}\text { H } & 4.611971 & 2.131521 & -3.669887\end{array}$ $\begin{array}{lllll}\text { H } & 4.125532 & 0.539426 & -4.302828\end{array}$ B 2.3211961 .0692222 .683562 F $0.9147621 .294042 \quad 2.831340$ F $2.9986561 .664092 \quad 3.781022$ F $2.557375-0.3372542 .666422$ H $4.2643303 .476458-1.958451$ H $4.600302 \quad 4.538596 \quad 0.255519$ H $3.740758 \quad 3.411335 \quad 2.316447$ H $-4.586988-1.9077190 .406599$ 76

23 R=CHCNEt ET/p

Ni $0.092248 \quad-0.015593 \quad 0.099160$ $\begin{array}{llll}\mathrm{N} 2 & 2.070869 & -0.017978 & 0.319251\end{array}$ $\mathrm{N} 0.476895 \quad 1.934963 \quad 0.061833$ $\begin{array}{llll}\text { C } & 1.707582 & 2.284418 & 0.298142\end{array}$ $\begin{array}{llll}\text { C } 2.671740 & 1.155398 & 0.265327\end{array}$ $\begin{array}{llll}\text { C }-0.212731 & -2.027249 & 0.321855\end{array}$ C $-0.794659-2.291803 \quad 1.722361$ $\begin{array}{llll}\text { C }-0.844790 & -3.821828 & 2.020358\end{array}$ $\begin{array}{llll}\text { C }-0.985180 & -2.706086 & -0.670344\end{array}$ $\mathrm{N}-1.658310-3.281941-1.432262$ H $0.831837-2.372143 \quad 0.238796$ H $-1.801479-1.8722651 .793752$ H $-0.181891-1.7962972 .469904$ $\begin{array}{llll}\mathrm{H} & -0.181891 & -1.796297 & 2.469904\end{array}$ $\begin{array}{llll}\mathrm{H} & -1.528368 & -4.323666 & 1.33513\end{array}$ H $0.142423-4.269886 \quad 1.915085$ H $-1.203074-3.9692573 .051594$ $\begin{array}{llll}C & -1.546828 & 0.067773 & -1.056673\end{array}$ $\begin{array}{llll}C & -0.401344 & -0.160315 & -1.902841\end{array}$ H $-0.114463-1.135871-2.271352$ H $0.156178 \quad 0.666578 \quad-2.357609$ H $-2.244619-0.753468-0.932265$ H $-1.962931 \quad 1.073208-1.023745$ $\begin{array}{lll}\text { C } 2.808528 & -1.265282 & 0.216742\end{array}$ $\begin{array}{llll}\text { C } & -0.656051 & 2.825820 & 0.085649\end{array}$ $\begin{array}{llll}\text { C } 2.046546 & 3.772098 & 0.486477\end{array}$ C $4.185944 \quad 1.280593 \quad-0.079391$ F $4.2960591 .376121-1.405001$ F 4.8040922 .3865520 .381316 $\begin{array}{llll}\text { F } & 4.894440 & 0.240529 & 0.365377\end{array}$ F $1.993873 \quad 4.331783-0.734232$ F $3.2793354 .069790 \quad 0.914553$ $\begin{array}{llll}\text { F } & 3.279335 & 4.069790 & 0.914553 \\ \text { F } & 1.229563 & 4.413861 & 1.328799\end{array}$ $\begin{array}{llll}\mathrm{F} & 1.229563 & .413861 & 1.328799\end{array}$ C $-1.051623 \quad 3.556020-1.058832$ $\begin{array}{llll}C & -1.051623 & 3.556020 & -1.058832\end{array}$ $\begin{array}{llll}\text { C } & -2.636916 & 3.470364 & 1.277254 \\ \text { C } & -2.263163 & 4.258117 & -0.967690\end{array}$ $\begin{array}{llll}\text { C } & -2.263163 & 4.258117 & -0.96769 \\ \text { C }-3.050162 & 4.222042 & 0.177894\end{array}$ $\begin{array}{llll}\text { C }-3.050162 & 4.222042 & 0.177894 \\ \text { B }-1.009960 & 1.816777 & 2.512314\end{array}$ F $0.248342 \quad 2.166312 \quad 3.048923$ F $-1.991288 \quad 1.740195 \quad 3.483077$ F $-0.834997 \quad 0.416224 \quad 1.955139$ C $-0.277772 \quad 3.601144 \quad-2.356354$ C $0.105853 \quad 5.053169-2.743360$ C $-1.079730 \quad 2.995338 \quad-3.539516$ н $0.639359 \quad 3.019811-2.252493$ H $0.5862295 .577244-1.903049$ H $0.5862295 .577244-1.903049$ H $-0.784398-5.626733-3.032627$ H $0.802358-5.041386-3.595379$ H $-1.9730073 .590819-3.744729$ $\mathrm{H}-1.3924301 .961568-3.331361$ H -0.449430 H -3.2542593 .4148702 .173628$ $\begin{array}{lllll}\mathrm{H} & -3.994412 & 4.771679 & 0.202112\end{array}$ H $-2.5952214 .830917-1.828500$ C $3.144609-1.759394-1.051308$ $\begin{array}{llll}\text { C } 3.023637 & -1.956075 & 1.426574\end{array}$ c $3.745846-3.021707-1.051399$ $\begin{array}{llll}\text { C } 3.638818 & -3.205742 & 1.336622\end{array}$ $\begin{array}{llll}\text { C } 3.996653 & -3.747878 & 0.106984\end{array}$ $\begin{array}{llll}\text { C } 2.677310 & -1.352895 & 2.778875\end{array}$ C $2.245181-2.4165413 .837680$ C $3.849178-0.540840 \quad 3.353173$ $\begin{array}{llll}\text { H } & 1.831059 & -0.662616 & 2.640552\end{array}$ $\begin{array}{llll}\text { H } & 1.831059 & -0.662616 & 2.640552 \\ \text { H } & 1.486002 & -3.106040 & 3.438407\end{array}$ $\begin{array}{llll}\text { H } & 1.486002 & -3.106040 & 3.438407 \\ \text { H } 3.110847 & -3.012158 & 4.175993\end{array}$ $\begin{array}{llll}H & 3.110847 & -3.012158 & 4.175993 \\ H & 1.825938 & -1.902471 & 4.719093\end{array}$ $\begin{array}{lllll}\text { H } & 1.825938 & -1.902471 & 4.719093 \\ \text { H } & 4.134320 & 0.295206 & 2.701990\end{array}$ $\begin{array}{lllll}\text { H } & 4.134320 & 0.295206 & 2.701990 \\ \text { H } & 4.733049 & -1.187113 & 3.471993\end{array}$ $\begin{array}{lllll}\text { H } & 4.733049 & -1.187113 & 3.471993 \\ \text { H } & 3.576711 & -0.130004 & 4.338263\end{array}$ B $2.900688-1.106101-2.55271$ F $4.144613-1.052188-3.21809$ F $2.012190-1.964254-3.258934$ $\begin{array}{lllll}\text { H } 3.833815 & -3.773085 & 2.258574\end{array}$ H $4.463980 \quad-4.736115 \quad 0.057077$ H $4.021091-3.447345-2.031774$ $\mathrm{F}$

$23 \mathrm{R}=$ CHCNEt $\mathrm{AC} / \mathrm{pi}$

Ni $0.139540-0.004862-0.154142$ $\mathrm{N} 2.157967-0.118320-0.073391$ $\begin{array}{llll}N & 0.157967 & -0.118320 & -0.073391\end{array}$ $\begin{array}{llll}\mathrm{N} & 0.418377 & -2.027090 & -0.142657 \\ \mathrm{C} & 1.658477 & -2.396277 & -0.169156\end{array}$ $\begin{array}{llll}\text { C } 2.625113 & -1.318705 & 0.088782\end{array}$ $\begin{array}{llll}\text { C } & 2.625113 & -1.318705 & 0.088782\end{array}$ $\begin{array}{llll}\text { C } & 0.163153 & 2.024912 & -0.342234 \\ \text { C } & 0.083749 & 2.382372 & -1.830649\end{array}$ $\begin{array}{llll}\text { C } & 0.083749 & 2.382372 & -1.830649 \\ \text { C } & 0.540199 & 3.834491 & -2.041444\end{array}$ $\begin{array}{lllr}\text { C } & 0.540199 & 3.834491 & -2.041444 \\ \text { C } & -0.771592 & 2.777446 & 0.435622\end{array}$ $\begin{array}{llll}\text { C }-0.771592 & 2.777446 & 0.435622\end{array}$ $\begin{array}{lllll}\mathrm{N} & -1.529035 & 3.451587 & 1.016422\end{array}$ H $1.159093 \quad 2.201541 \quad 0.090756$ H $-0.941343 \quad 2.231973 \quad-2.200988$ H $0.727901 \quad 1.706359 \quad-2.404397$ H $1.578484 \quad 3.976943 \quad-1.694093$ H $-0.100764 \quad 4.533111-1.485509$ н $0.484004 \quad 4.089113-3.112840$ C $-0.632028 \quad 0.134292 \quad 1.718715$ C $-1.676049 \quad-0.183649 \quad 0.827476$ $\begin{array}{llll}\text { C }-2.749028 & 0.652218 & 0.427162\end{array}$ $\begin{array}{llll}\mathrm{N} & -3.722120 & 1.197631 & 0.065182\end{array}$ $\begin{array}{llll}\mathrm{H} & -1.932818 & -1.232084 & 0.655053 \\ \mathrm{H} & -0.125833 & -0.673789 & 2.245207\end{array}$

H $-0.504575 \quad 1.1249312 .146949$ C $3.077175 \quad 1.003909-0.067337$ $\begin{array}{llll}\text { C }-0.699069 & -2.882325 & -0.418554\end{array}$ $\begin{array}{llll}\text { C } 2.083718 & -3.834740 & -0.523204\end{array}$ $\begin{array}{llll}\text { C } 3.940038 & -1.654977 & 0.852253\end{array}$ F $3.569112-2.5667331 .777374$ F $4.452465-0.6620701 .559679$ F $4.955041-2.1979110 .135902$ F $3.430821-3.922261-0.502721$ $\begin{array}{lllll}\text { F } 1.634321 & -4.768746 & 0.332432\end{array}$ $\begin{array}{llll}\text { F } & 1.634321 & -4.768746 & 0.332432 \\ \text { C } & 1.634435 & -4.218255 & -1.738968\end{array}$ $\begin{array}{llll}\mathrm{F} & 1.634435 & -4.218255 & -1.738968 \\ \mathrm{C} & -1.305414 & -2.662064 & -1.672304\end{array}$ $\begin{array}{llll}\text { C } & -1.305414 & -2.662064 & -1.672304 \\ \text { C }-1.261935 & -3.698516 & 0.588437\end{array}$ $\begin{array}{llll}\text { C }-2.508175 & -3.321634 & -1.920360\end{array}$ $\begin{array}{llll}C & -2.508175 & -3.321634 & -1.920360\end{array}$ $\begin{array}{llll}C & -2.479041 & -4.320632 & 0.274311\end{array}$ $\begin{array}{llll}\text { C }-3.103902 & -4.138185 & -0.955274\end{array}$ B $-0.683632-1.626883-2.750100$ F $-0.651744-0.266744-2.073466$ F $0.647974-1.898429-3.089826$ F $-1.501007-1.481997-3.869818$ $\begin{array}{llll}\text { C } & -0.681637 & -3.894965 & 1.982895\end{array}$ $\begin{array}{llll}\text { C }-0.479246 & -5.392514 & 2.315502\end{array}$ C $-1.568344-3.274256 \quad 3.071128$ H $0.298789-3.4015912 .025686$ H $0.076099-5.924510 \quad 1.531626$ H $0.079227 \quad-5.489086 \quad 3.263324$ H $-1.448092-5.906308 \quad 2.444845$ $\begin{array}{llll}\mathrm{H}-1.448092 & -5.906308 & 2.444845\end{array}$ $\begin{array}{llll}\text { H }-1.756779 & -2.206267 & 2.902777 \\ \text { H }-1.082885 & -3.386068 & 4.053225\end{array}$ $1-1.082885-3.3860684 .053225$ $\mathrm{H}-2.545134-3.7827163 .124089$ $\begin{array}{llll}\mathrm{H}-2.992022 & -3.157805 & -2.882437\end{array}$ H $-4.062967-4.615593-1.152894$ $\begin{array}{lllll}\text { H }-2.960628 & -4.946961 & 1.02685\end{array}$ $\begin{array}{llll}\text { C } 3.661240 & 1.313646 & -1.324894\end{array}$ C 3.3044251 .7430121 .097900 C $4.5723952 .336692-1.340950$ $\begin{array}{llll}\text { C } 4.256057 & 2.784664 & 0.992826\end{array}$ $\begin{array}{lllll}\text { C } 4.888795 & 3.066244 & -0.208897\end{array}$ $\begin{array}{llll}\text { C } 3.311976 & 0.539091 & -2.592269\end{array}$ $\begin{array}{llll}\text { C } 3.179232 & 1.452473 & -3.816748\end{array}$ $\begin{array}{llll}\text { C } 4.337347 & -0.556988 & -2.912087\end{array}$ H $2.3470210 .044636-2.443083$ $\begin{array}{llll}\mathrm{H} & 2.539324 & 2.323800 & -3.616542\end{array}$ $\begin{array}{llll}\mathrm{H} & 2.729392 & 0.881416 & -4.639464\end{array}$ $\begin{array}{llll}\text { H } & 4.154227 & 1.820413 & -4.161120 \\ \text { H } & 4.440787 & -1.292393 & -2.101761\end{array}$

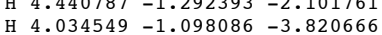
$\begin{array}{llll}\text { H } & 4.034549 & -1.098086 & -3.820666 \\ \text { H } & 5.336259 & -0.116329 & -3.079492\end{array}$ $\begin{array}{llll}\text { H } & 5.336259 & -0.116329 & -3.079492 \\ \text { B } & 2.527625 & 1.543149 & 2.539355\end{array}$ $\begin{array}{llll}\text { B } & 2.527625 & 1.543149 & 2.539355 \\ \text { F } & 1.280605 & 2.199621 & 2.454902\end{array}$ $\begin{array}{llll}\text { F } 1.280605 & 2.199621 & 2.454902 \\ \text { F } 3.222981 & 2.174110 & 3.576324\end{array}$ $\begin{array}{llll}\text { F } & 3.222981 & 2.174110 & 3.576324 \\ \text { F } 2.297487 & 0.153551 & 2.811964\end{array}$ H $5.0592032 .589522-2.292263$ H $5.627773 \quad 3.872251-0.254195$ H

$23 \mathrm{R}=\mathrm{CHCNE}$ AC $/ \mathrm{N}$

Ni $0.029492-0.546723-0.318159$ $\mathrm{N} 1.1808661 .001537-0.048905$ $\mathrm{N} 1.1808611 .001537-0.048905$ $\mathrm{N}-1.3025110 .822076-0.509625$ C $0.6048512 .041231-0.390863$ $\begin{array}{llll}C & 0.604430 & 2.143254 & -0.269073 \\ \text { C } & -3.771573 & 2.107064 & -3.570171\end{array}$ $\begin{array}{lllll}\text { C } & 2.576228 & 0.876279 & 0.308234\end{array}$ $\begin{array}{llll}\text { C } & 2.576228 & 0.876279 & 0.308234 \\ \text { C }-2.713891 & 0.514626 & -0.457124\end{array}$ $\begin{array}{llll}\text { C } & -2.713891 & 0.514626 & -0.457124 \\ \text { C } & -1.744029 & 3.289774 & -0.288917\end{array}$ C $1.365802 \quad 3.464777-0.405850$ F 1.4906924 .0878810 .800578 F $2.5918783 .312896-0.913675$ F $0.6842494 .294534-1.243167$ F $-1.876736 \quad 3.856717-1.525994$ F $-1.160674 \quad 4.188791 \quad 0.524092$ $\begin{array}{lllll}F & -2.965455 & 3.095227 & 0.212998\end{array}$ C $-3.192445 \quad 0.121762 \quad 0.806103$ C $-3.4858750 .521020-1.629226$ C $-4.531679-0.2704520 .865149$ $\begin{array}{llll}C & -4.531679 & -0.270452 & 0.865149\end{array}$ $\begin{array}{llll}C & -4.819036 & 0.108573 & -1.499703\end{array}$ $\begin{array}{llll}C & -5.339812 & -0.284974 & -0.272431\end{array}$ B -2.2482080 .1588142 .139416$ F $-2.940194-0.3431843 .257297$ F $-1.071898-0.679556 \quad 1.912129$ $\begin{array}{llll}F & -1.770994 & 1.474910 & 2.385247\end{array}$ $\begin{array}{lllll}\text { C } & -2.951835 & 0.944571 & -2.987527\end{array}$ $\begin{array}{llll}\text { C }-2.919080 & -0.224772 & -3.982907\end{array}$ H $-1.9156821 .293146-2.860099$ H $-3.8566902 .940076-2.858817$ H $-3.293360 \quad 2.478202-4.489943$ H $-4.789055 \quad 1.774318-3.830630$ н $-2.269372-1.041722-3.641785$ н $-2.5324490 .122850-4.953809$ H $-3.931690-0.629715-4.141452$ $\mathrm{H}-4.933371-0.5671201 .835819$ H $-6.382100-0.601980-0.203966$ 
$\begin{array}{llll}\text { C } 4.861481 & 0.497488 & -0.235090\end{array}$ C 5.1921210 .5 C $1.7849700 .980722 \quad 2.765099$ $\begin{array}{llll}\text { C } 1.624798 & -0.350948 & 3.507261\end{array}$ C $2.0686192 .104471 \quad 3.769463$ H $0.816451 \quad 1.200690 \quad 2.296199$ H $1.403320-1.169706 \quad 2.808151$ H $2.546042-0.600840 \quad 4.059850$ $\begin{array}{lllll}\text { H } & 0.788800 & -0.285601 & 4.219351\end{array}$ H 2.2125 H 2.2125733 .0713373 .260425 $\begin{array}{llll}\text { H } 2.973225 & 1.892701 & 4.365763\end{array}$ $\begin{array}{lllll}\text { H } & 1.217744 & 2.200582 & 4.464692\end{array}$ B $3.2801490 .622766-2.310161$ F $4.0717591 .608957-2.944467$ $\begin{array}{llll}\text { F } & 1.905517 & 0.815352 & -2.612747 \\ \text { F } 3.695240 & -0.670864 & -2.791723\end{array}$ $\begin{array}{llll}\text { F } & 3.695240 & -0.670864 & -2.791723 \\ \text { H } & 4.442908 & 0.692172 & 3.128434\end{array}$ $\begin{array}{llll}\mathrm{H} & 4.442908 & 0.692172 & 3.128434 \\ \mathrm{H} & 6.229784 & 0.379972 & 1.437885\end{array}$ $\begin{array}{llll}\text { H } & 6.229784 & 0.379972 & 1.437885 \\ \text { H } & 5.642640 & 0.362528 & -0.989122\end{array}$ $\begin{array}{llll}\text { H } & 5.642640 & 0.362528 & -0.989122 \\ \text { C }-1.125275 & -2.062680 & -0.934941\end{array}$ C $-1.207799 \quad-3.172714 \quad 0.122316$ C $-2.359426-4.141415-0.171840$ H $-2.108154-1.580034-1.056349$ C $-0.675191-2.544773-2.209428$ H $-0.253946-3.721010 \quad 0.152790$ H -1.359670 $\begin{array}{llll}\mathrm{H}-1.359670 & -2.701394 & 1.103270\end{array}$ $\begin{array}{llll}\mathrm{H} & -3.323416 & -3.609050 & -0.163658\end{array}$ $\begin{array}{llll}\mathrm{H} & -2.235749 & -4.618482 & -1.156536\end{array}$ $\mathrm{H}-2.399554-4.930363-0.595355$ $\mathrm{N} 1.424036-1.700919-0.375715$ C $2.412089-2.281930-0.566685$ $\begin{array}{llll}\text { C } 3.634561-2.911266 & -0.877118\end{array}$ $\begin{array}{llll}C & 4.057918 & -4.021129 & -0.253973\end{array}$ H $4.182901-2.397753-1.675494$ H $5.011304-4.473723-0.531970$ H $3.477790 \quad-4.494407 \quad 0.541030$ $\mathrm{N}-0.329612-2.983238-3.238490$

81

$\begin{array}{llll}24 \mathrm{R}=\mathrm{Me} & 036585 & -0.076946 & 0.038639\end{array}$ $\mathrm{N} 1.814612$ C 2.484169 c $2.484169-0.024258-0.049794$ C $2.429683-1.1927880 .003$ C $3.874211-1.3778390 .566490$ $\begin{array}{llll}C & 3.941410 & -0.643074 & 1.928833\end{array}$

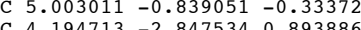
$\begin{array}{llll}\text { C } 4.194713 & -2.847534 & 0.893886\end{array}$ $\begin{array}{llll}\text { C } 1.461865 & -2.331939 & -0.187792\end{array}$ C $1.707581-3.529796-1.160109$ $\begin{array}{llll}\text { C } 2.826549 & -3.149191 & -2.153852\end{array}$ c $1.997974-4.905701-0.524696$ $\begin{array}{llll}\text { C } 0.445895 & -3.715962 & -2.040077\end{array}$ $\begin{array}{llll}\mathrm{N} & 0.273113 & -2.001509 & 0.273917\end{array}$ C $-0.772159 \quad-2.934738 \quad 0.535753$ $\begin{array}{llll}\text { C }-2.038048 & -2.738300 & -0.053512\end{array}$ $\begin{array}{llll}\text { C }-0.564282 & -3.939969 & 1.517598\end{array}$ $\begin{array}{llll}C & -3.037368 & -3.678353 & 0.212838\end{array}$ $\begin{array}{llll}C & -1.599799 & -4.853212 & 1.735551\end{array}$ $\begin{array}{llll}C & -2.815271 & -4.757275 & 1.062607\end{array}$ $\begin{array}{llll}\text { B }-2.405095 & -1.408238 & -0.885507\end{array}$ F $-1.709167-1.231956-2.094124$ F $-3.778818-1.245655-1.064762$ F $-1.959905-0.2709570 .038764$ $\begin{array}{llll}\text { C } & 0.644959 & -3.932207 & 2.441527\end{array}$ $\begin{array}{llll}\text { C } & 0.943174 & -5.283747 & 3.096559\end{array}$ $\begin{array}{llll}\text { C } 0.464269 & -2.844374 & 3.515153\end{array}$ H $1.530284-3.660884 \quad 1.852181$ $\begin{array}{llll}\text { H } & 1.021706 & -6.096412 & 2.357717\end{array}$ H $1.895715 \quad-5.222694 \quad 3.645646$ H $0.165938-5.555424 \quad 3.828281$ H $0.360191-1.8438563 .070670$ H $-0.440004-3.051703 \quad 4.111182$ H $1.333544-2.827530 \quad 4.192545$ $\begin{array}{lllll}\mathrm{H} & -4.015609 & -3.528947 & -0.246723 \\ \mathrm{H} & -3.600018 & -5.493126 & 1.246767\end{array}$ $\begin{array}{llll}\mathrm{H} & -3.600018 & -5.493126 & 1.24676\end{array}$ $\begin{array}{llll}\text { H } & -1.461252 & -5.646246 & 2.469894 \\ \text { C } 3.094622 & 1.701560 & -1.219223\end{array}$ $\begin{array}{llll}\text { C } & 3.094622 & 1.701560 & -1.219223 \\ \text { C } & 3.649764 & 2.077845 & 1.114033\end{array}$ $\begin{array}{llll}\text { C } 3.643229 & 2.988947 & -1.228091\end{array}$ $\begin{array}{llll}\text { C } & 2.643229471 & 2.9889475 & -1.228091 \\ \text { C } & 3.573992 & 3.85345 & 1.039953\end{array}$ $\begin{array}{llll}\text { C } & 2.929471 & 3.353345 & 1.039953 \\ \text { C } 3.573992 & 3.813146 & -0.102961\end{array}$ $\begin{array}{llll}\text { C } 3.573992 & 3.813146 & -0.102961 \\ \text { C } 3.108288 & 0.875740 & -2.498040\end{array}$ $\begin{array}{llll}\text { C } 3.108288 & 0.875740 & -2.498040 \\ \text { C } 4.376535 & 1.115280 & -3.374859\end{array}$ $\begin{array}{llll}\text { C } & 4.376535 & 1.115280 & -3.374859 \\ \text { C } & 1.846101 & 1.112704 & -3.341301\end{array}$ $\begin{array}{lllll}\text { C } 1.846101 & 1.112704 & -3.341301 \\ & & \end{array}$ H $3.118144-0.185681-2.21949$ $\begin{array}{llll}\text { H } & .355740 & 2.114154 & -3.841910\end{array}$ H $5.3029711 .026327-2.784152$ H $0.937396 \quad 0.825473-2.794793$ H $1.892126 \quad 0.520856-4.270623$ H $1.7617042 .178026-3.612161$ $\begin{array}{llll}\text { B } 1.7617692 & 2.178026 & -3.612161\end{array}$ 1.5836921 .7378642 .535902 F 0.6606262 .7798582 .805144 F 0.5871830 .4869792 .57994 H $4.125306 \quad 3.359778-2.135220$
H $4.009387 \quad 4.816403-0.134398$ н $2.841763 \quad 3.998990 \quad 1.916727$ C $-0.405024 \quad 1.696559-0.617180$ $\begin{array}{llllllll}\text { H } & -0.784557 & 2.173451 & 0.304924\end{array}$ н $-1.195508 \quad 1.596398-1.374929$ H $0.477532 \quad 2.223840 \quad-0.996121$ H $4.867176-0.952337 \quad 2.441188$ H $3.965793 \quad 0.445850 \quad 1.822793$ H $3.081080-0.890708 \quad 2.565987$ $\begin{array}{llll}\text { H } 5.965549 & -1.054636 & 0.160210\end{array}$ $\begin{array}{llll}\text { H } & 5.017946 & -1.316654 & -1.32522\end{array}$ $\begin{array}{lllll}\text { H } & 4.928641 & 0.248308 & -0.462832\end{array}$ $-5.154383-2.8651871 .433758$ H $3.438168-3.2867901 .560081$ H $4.308670-3.4856690 .011320$ H $2.947857-3.972825-2.875892$ $\begin{array}{llll}\text { H } 2.542880 & -2.247681 & -2.719204\end{array}$ H $3.800166-2.971386-1.686580$ H $0.722047-4.355902-2.894096$ H $-0.369382-4.214075-1.500363$ H $0.065071-2.757764-2.421146$ H $2.147339-5.632557-1.341280$ H $2.890030-4.9256970 .112255$ $\mathrm{H}$
87

$24 \mathrm{R}=\mathrm{Me} \mathrm{ET} / \mathrm{pi}$

$\mathrm{Ni} 0.190070-0.113320-0.074223$ N $2.176333-0.1214620 .047649$ $\begin{array}{llll}\mathrm{N} & 2.176333 & -0.121462 & 0.047649\end{array}$ $\begin{array}{llll}\text { C } 1.799675 & -2.446643 & -0.104617\end{array}$ $\begin{array}{llll}\text { C } 2.754397 & -1.280336 & 0.253755\end{array}$ C $-0.0718591 .782716-0.425812$ H $0.808745 \quad 2.063693 \quad-1.019766$ H $-0.993127 \quad 1.837324-1.02354$ $\begin{array}{lllll}\text { H } & -0.120535 & 2.361899 & 0.503273\end{array}$ C $-1.628484 \quad-0.089676 \quad 0.821045$ $\begin{array}{llll}\text { C }-0.593067 & -0.007350 & 1.762673\end{array}$ H $-0.229446-0.899277 \quad 2.278879$ H $-2.123926-1.038049 \quad 0.590509$ H $-2.192796 \quad 0.808576 \quad 0.560903$ $\begin{array}{lllll}\mathrm{H} & -0.305259 & 0.939367 & 2.222553\end{array}$ B $-0.508515-1.633698-2.788333$ F $-0.247085-0.321808-2.173541$ F $-0.247085-0.321808-2.173541$ F $0.746593-2.125018-3.243774$ F $-1.368150-1.427828-3.880142$ C 2.8606681 .1507 $\begin{array}{llll}C & -0.618833 & -2.833950 & -0.397991\end{array}$ C $-1.184710-2.605225-1.672548$ $\begin{array}{llll}\text { C } 2.304528 & -3.806777 & -0.672516\end{array}$ C $4.108813-1.4902351 .015071$ $\begin{array}{llll}\text { C } 4.865305 & -0.194959 & 1.377971\end{array}$ $\begin{array}{llll}\text { C } 5.201358 & -2.344773 & 0.326015\end{array}$ $\begin{array}{lll}\text { C } 3.683566 & -2.101720 & 2.379900\end{array}$ $\begin{array}{llll}\text { C } 3.281277 & -3.483294 & -1.838182\end{array}$ $\begin{array}{llll}\text { C } 2.910752 & -4.725123 & 0.417487\end{array}$ $\begin{array}{llll}\text { C } 1.230315 & -4.695007 & -1.340948\end{array}$ $\begin{array}{llll}\text { C }-1.265517 & -3.616887 & 0.579047\end{array}$ C $-2.388603-3.247290-1.966029$ C -2.473523 C $-3.033080-4.064149-1.037317$

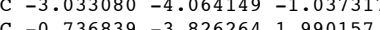
C $-0.736839-3.8262641 .990157$ C $-0.488515-5.3129192 .296028$ c $-1.695208-3.2475553 .045116$ H $0.222764-3.2944482 .079487$ H $0.166268-5.7850721 .549903$ $\begin{array}{llll}\mathrm{H} & -0.025864 & -5.427207 & 3.289521\end{array}$ H $-1.438453-5.871492 \quad 2.302397$ H $-1.902220-2.1840602 .866858$ H $-1.263158-3.354015 \quad 4.053196$ H $-2.656720-3.785992 \quad 3.028620$ H $-2.824265-3.080241-2.952789$ н $-3.975943-4.553945-1.287223$ H $-2.987763 \quad-4.851101 \quad 0.959190$ C $3.345304 \quad 1.432105-1.366718$ $\begin{array}{llll}\text { C } 2.892744 & 2.109431 & 0.972015\end{array}$ $\begin{array}{llll}\text { C } 3.972424 & 2.663852 & -1.582249\end{array}$ c $3.9724242 .663852-1.582249$ C $3.536302 \quad 3.3198660 .680375$ $\begin{array}{llll}C & 4.088722 & 3.602568 & -0.565421\end{array}$ C $3.1716580 .499931-2.560192$ C $2.3402191 .157837-3.673283$ $\begin{array}{llll}\text { C } & 4.520582 & 0.022566 & -3.116475 \\ \mathrm{H} & 2.605581 & -0.384597 & -2.244509\end{array}$ H $1.3601691 .476352-3.293141$ H $2.162015 \quad 0.432443-4.481955$ H $2.8580482 .035216-4.094568$ H $5.129810-0.461555-2.337895$ H $4.361046-0.698564-3.93404$ H $5.105386 \quad 0.867902 \quad-3.515534$ B $2.238024 \quad 2.027491 \quad 2.486176$ $\begin{array}{lllll}\text { F } 0.903141 & 2.483518 & 2.463186\end{array}$ F $2.863727 \quad 2.931958 \quad 3.368176$ F $2.283727 \quad 0.6996733 .019757$ $\begin{array}{llll}4 & 4.360779 & 2.889965 & -2.578017\end{array}$ - $3.5860692 .559016-0.752201$ $3.753477-4.3137680 .974458$ $\begin{array}{llll}\text { H } 3.753477 & -4.313768 & 0.974458 \\ \text { H } 3.247410 & -5.654443 & -0.071172\end{array}$

H $2.131182-4.997945 \quad 1.144980$ H $3.905776-4.368251-2.047336$ H $2.686755-3.244701-2.73132$ н $3.941465-2.632258-1.65461$ H $1.774112-5.551216-1.775779$ H $0.487854-5.094939-0.639383$ H $0.717007-4.177072-2.157803$ H $4.916009-3.349306 \quad 0.015817$ H $6.029840-2.444285 \quad 1.045708$ H $5.598945-1.816205-0.554660$ $\begin{array}{llll}H & 5.598945 & -1.816205 & -0.554660\end{array}$

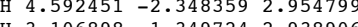
$\begin{array}{llll}\text { H } 3.106898 & -1.349724 & 2.938906\end{array}$ $\begin{array}{lll}\mathrm{H} & 3.077731-3.011548 & 2.288676\end{array}$ $\begin{array}{llll}\text { H } 5.766322 & -0.505352 & 1.933392\end{array}$ $\begin{array}{lllll}\text { H } & 5.193732 & 0.369203 & 0.493956\end{array}$ $\begin{array}{llll}\text { H } & 4.280992 & 0.455570 & 2.032567\end{array}$

88

$24 \mathrm{R}=\mathrm{Me} \mathrm{AC} / \mathrm{pi}$

Ni $0.192131-0.073758-0.101253$ $\begin{array}{llll}\text { N } 2 & 2.170544 & -0.092496 & 0.055832\end{array}$

$\begin{array}{llll}\mathrm{N} & 0.543524 & -2.056625 & -0.068049\end{array}$

$\begin{array}{llll}\text { C } 1.788453 & -2.423219 & -0.099564\end{array}$

$\begin{array}{llll}\text { C } 2.734643 & -1.254031 & 0.276269\end{array}$

C $-0.019094 \quad 1.824363-0.492979$ н $0.874358 \quad 2.059305-1.086287$

$\mathrm{H}-0.940297-1.873430-1.089143$

$4-0.0567172 .419194-0.426036$

$\begin{array}{llll}H & -0.056717 & 2.419194 & 0.426036\end{array}$

$\begin{array}{llll}C & -1.624139 & -0.102848 & 0.846307\end{array}$

C -0.5305020 .0734221 .730574$

$\begin{array}{llll}\mathrm{H} & -0.121519 & -0.791097 & 2.257006\end{array}$

$\mathrm{H}-2.035156-1.1012140 .659150$

$\begin{array}{lllll}C & -2.577676 & 0.930103 & 0.608096\end{array}$

H $-0.319328 \quad 1.043331 \quad 2.183356$

B $-0.560544-1.614608-2.780102$

$\begin{array}{llll}F & -0.207435 & -0.316982 & -2.163457\end{array}$ F $0.653276-2.149989-3.292159$

F $-1.453536-1.355909-3.826680$ $\begin{array}{llll}\text { C } 2.901941 & 1.152949 & -0.071159\end{array}$ C $-0.629039-2.817578-0.388349$ $\begin{array}{llll}\text { C }-1.225118 & -2.572091 & -1.645400\end{array}$ $\begin{array}{llll}\text { C } 2.306758 & -3.765695 & -0.698970\end{array}$ C $4.064677-1.4757711 .068527$ C $4.783568-0.190180 \quad 1.547687$ C $5.205698-2.2443550 .359368$ $\begin{array}{llll}C & .205698 & -2.244355 & 0.359368\end{array}$ $\begin{array}{lll}C & 3.600175-2.169711 & 2.380706\end{array}$ C $3.256863-3.391842-1.867303$ $\begin{array}{llll}C & 2.971796 & -4.704792 & 0.335682\end{array}$ C $1.230765-4.650430-1.366315$ C $-1.249802-3.6083280 .598940$ $\begin{array}{llll}\text { C }-2.444590 & -3.197756 & -1.908505\end{array}$ $\begin{array}{llll}C & -2.473962 & -4.207093 & 0.271560\end{array}$ $\begin{array}{llll}\text { C }-3.070115 & -4.015705 & -0.967766\end{array}$ $\begin{array}{llll}\text { C }-0.675261 & -3.836193 & 1.989087\end{array}$ $\begin{array}{llll}\text { C }-0.427532 & -5.328086 & 2.267667\end{array}$ C $-1.587905-3.257235 \quad 3.083053$ H $0.292204 \quad-3.315273 \quad 2.051004$ н $0.203307 \quad-5.791684 \quad 1.495878$ H $0.063336-5.4586943 .245368$ H $-1.379415-5.8823452 .293389$ $\begin{array}{llll}\mathrm{H} & -1.379415 & -5.882345 & 2.293389 \\ \mathrm{H}-1.780848 & -2.186548 & 2.931293\end{array}$ $\begin{array}{llll}H & -1.780848 & -2.186548 & 2.931293\end{array}$ $\begin{array}{llll}H & -1.126493 & -3.388714 & 4.074891\end{array}$ H $-2.560398-3.7751543 .089189$ $\mathrm{H}-2.910348-3.013959-2.878212$ H $-4.028481-4.486862-1.192136$ $\begin{array}{lllll}H & -2.973066 & -4.829693 & 1.01611\end{array}$ $\begin{array}{llll}\text { C } 3.459899 & 1.370275 & -1.354750\end{array}$ $\begin{array}{llll}\text { C } 2.929069 & 2.135236 & 0.943852\end{array}$ $\begin{array}{llll}\text { C } 4.153708 & 2.566210 & -1.569857\end{array}$ C $3.6404673 .306690 \quad 0.652118$ $\begin{array}{llll}\text { C } 4.264308 & 3.525860 & -0.572957\end{array}$ $\begin{array}{llll}\text { C } 3.270655 & 0.431793 & -2.541193\end{array}$ $\begin{array}{llll}\text { C } 2.409679 & 1.084178 & -3.631381\end{array}$ C $4.599292-0.029392-3.154729$ H $2.724514-0.459903-2.212715$ H $1.4313221 .390055-3.237553$ H $2.226596 \quad 0.363221-4.443096$ $\begin{array}{lllll}\text { H } & 2.226596 & 0.363221 & -4.443096 \\ \text { H } & 2.912511 & 1.970534 & -4.052161\end{array}$ H $5.259914-0.494140-2.404242$ $\begin{array}{llll}\text { H } & .259914 & -0.494140 & -2.404242 \\ \text { H } 4.408903 & -0.765379 & -3.954780\end{array}$ $\begin{array}{llll}\text { H } & 5.149075 & 0.818041 & -3.600630\end{array}$ B 2.2184422 .1040762 .435581 F $0.9036092 .606374 \quad 2.361396$ F $2.854556 \quad 2.9909873 .323911$ F 2.1920880 .7783532 .985163 H $4.5990602 .747336-2.550657$ H $4.817200 \quad 4.451239-0.759747$ H $3.700982 \quad 4.061980 \quad 1.439243$ H $3.834295 \quad-4.295970 \quad 0.868205$ н $3.303953-5.611398-0.200476$ H $2.230596 \quad-5.020750 \quad 1.088547$ H $3.837357-4.285535-2.158641$ H $2.647198-3.067918-2.724949$ 
H $6.023800 \quad-2.359101 \quad 1.091412$ H $5.599261-1.641331-0.47628$ H $4.490233-2.454616 \quad 2.966854$ H $3.011356-1.447822 \quad 2.967685$ H $2.992434-3.068687 \quad 2.222590$ H $5.640775 \quad-0.5264842 .156525$ H $5.176020 \quad 0.413628 \quad 0.717230$ H $4.144102 \quad 0.424373 \quad 2.186083$ $\begin{array}{llll}\mathrm{N} & -3.404628 & 1.737307 & 0.431512\end{array}$ 88

$4 \mathrm{R}=\mathrm{Me} \mathrm{AC} / \mathrm{N}$

Ni $0.000134-0.009244 \quad 0.103853$ N $1.894425 \quad 0.013442 \quad 0.087502$ N $0.368515-1.880537-0.222676$ C $1.602832-2.264541-0.414004$ C $2.558915-1.116361-0.017283$ C $-0.346920 \quad 1.9307310 .031941$ $\begin{array}{llll}\text { H } 0.564120 & 2.446253 & -0.286283\end{array}$ H $-1.1752932 .092491-0.671761$ H $-0.613652 \quad 2.198791 \quad 1.064627$ $\begin{array}{llll}\mathrm{N} & -1.769282 & -0.090409 & 0.403385\end{array}$ $\begin{array}{llll}\text { C } & -2.915209 & -0.118319 & 0.603485\end{array}$ $\begin{array}{llll}\text { C }-4.298094 & -0.108568 & 0.875882\end{array}$ $\begin{array}{llll}\text { C } & -5.161281 & -0.986392 & 0.33940\end{array}$ H $-4.6361630 .678506 \quad 1.555817$ H $-6.222359-0.9262390 .578908$ H $-4.817098-1.767598-0.339243$ B $-0.869442-1.399315-2.943708$ $\begin{array}{llll}\text { B }-0.869442 & -1.399315 & -2.943708 \\ \text { F }-1.091940 & -0.042170 & -2.562239\end{array}$ $\begin{array}{llll}\text { F } & -1.091940 & -0.042170 & -2.562239 \\ \text { F } & 0.534954 & -1.579453 & -3.162332\end{array}$ $\begin{array}{llll}\text { F } & 0.534954 & -1.579453 & -3.162332 \\ \text { F } & -1.564134 & -1.668534 & -4.153403\end{array}$ $\begin{array}{lllll}\text { F } & -1.564134 & -1.668534 & -4.153403 \\ \text { C } & 2.479831 & 1.278478 & 0.440124\end{array}$ $\begin{array}{llll}\text { C } & 2.479831 & 1.278478 & 0.440124 \\ \text { C }-0.828040 & -2.618568 & -0.509125\end{array}$ C $-1.443646-2.400843-1.760328$ C $1.979470-3.689720-0.950700$ $\begin{array}{llll}\text { C } 4.096087 & -1.234887 & 0.250504\end{array}$ C $4.908867-1.067485-1.101157$ $\begin{array}{llll}\text { C } 4.432453 & -2.553865 & 0.978194\end{array}$ $\begin{array}{lll}\text { C } 4.727516 & -0.185598 & 1.205735\end{array}$ $\begin{array}{llll}\text { C } 2.120449 & -4.677165 & 0.241066\end{array}$ C $3.232764-3.672007-1.853156$ C $0.923878-4.342235-1.884082$ C $-1.410189-3.3806490 .522605$ C $-2.647699-3.079634-1.980621$ $\begin{array}{llll}\text { C }-2.620572 & -4.023567 & 0.236707\end{array}$ C $-3.229629-3.893389-1.008540$ C $-0.789960-3.501994 \quad 1.908014$ $\begin{array}{llll}\text { C }-0.913812-4.918874 & 2.491792\end{array}$ C $-1.390758-2.4825492 .894150$ H $0.278451-3.264649 \quad 1.828148$ H $-0.581066-5.690146 \quad 1.779779$ H $-0.303196-4.998572 \quad 3.404139$ H $-1.953665-5.145752 \quad 2.772192$ H $-1.147414-1.4513402 .606165$ H $-0.972505 \quad-2.645067 \quad 3.899668$ H $-2.485739-2.598772 \quad 2.944115$ н $-3.120325-2.958542 \quad-2.956856$ H $-4.161257-4.426184-1.216180$ н $-3.088588 \quad-4.643683 \quad 1.002497$ C $2.9781862 .093096-0.595061$ $\begin{array}{llll}\text { C } 2.375092 & 1.684331 & 1.785558\end{array}$ $\begin{array}{llll}\text { C } 3.465847 & 3.355623 & -0.229136\end{array}$ $\begin{array}{llll}\text { C } 2.877714 & 2.952464 & 2.084222\end{array}$ C $3.4329993 .787664 \quad 1.094379$ C $2.9618791 .673030-2.059024$ C $1.6974242 .142452-2.796524$ $\begin{array}{llll}\text { C } 4.222022 & 2.167499 & -2.842534\end{array}$ H $2.9606090 .575460-2.106168$ H $0.793711 \quad 1.647764-2.418228$ $\mathrm{H} \quad 1.577746 \quad 3.234576 \quad-2.700626$ H $1.778286 \quad 1.889489-3.865880$ H $5.158703 \quad 1.949706-2.302910$ H $4.173023 \quad 3.255349-3.020723$ H $4.254884 \quad 1.670430 \quad-3.826970$ B $1.7104190 .775286 \quad 2.992569$ $\begin{array}{llll}\text { F } & 1.891696 & 1.438138 & 4.233548\end{array}$ $\begin{array}{llll}\text { F } & 1.891696 & 1.438138 & 4.233548 \\ \text { F } & 2.310165 & -0.525290 & 3.062273\end{array}$ $\begin{array}{llll}\text { F } & 2.310165 & -0.525290 & 3.062273 \\ \text { F } & 0.306839 & 0.593068 & 2.762136\end{array}$ $\begin{array}{llll}\text { F } & 0.306839 & 0.593068 & 2.762136 \\ \text { H } 3.873579 & 4.012899 & -0.999883\end{array}$ $\begin{array}{llll}\text { H } & 3.873579 & 4.012899 & -0.99988 \\ \text { H } 3.820390 & 4.777026 & 1.357119\end{array}$ $\begin{array}{llll}\text { H } & 3.820390 & 4.777026 & 1.357119 \\ \text { H } & 2.831361 & 3.287679 & 3.121933\end{array}$ $\begin{array}{llll}\text { H } & 2.831361 & 3.287679 & 3.121933 \\ \text { H } & 5.781081 & -0.492043 & 1.328268\end{array}$ $\begin{array}{llll}\mathrm{H} & 5.781081 & -0.492043 & 1.328268 \\ \mathrm{H} & 4.734409 & 0.833707 & 0.807550\end{array}$ $\begin{array}{llll}\text { H } & 4.734409 & 0.833707 & 0.807550 \\ \text { H } & 4.239586 & -0.193784 & 2.188605\end{array}$ $\begin{array}{llll}\text { H } & 4.239586 & -0.193784 & 2.188605 \\ \text { H } 5.877160 & -1.589536 & -0.99923\end{array}$ $\begin{array}{llll}\text { H } 5.877160 & -1.589536 & -0.99923 \\ \text { H } & 4.394284 & -1.447766 & -1.991670\end{array}$ H $5.1110310 .001318-1.260320$ н $5.508597 \quad-2.545387 \quad 1.212273$ H $3.884710-2.588021 \quad 1.933529$ H $4.232020-3.465437 \quad 0.417843$ H $3.383964-4.694060-2.234892$ H $3.047199-3.017802-2.719274$ . $4.047199-3.016802-2.719274$ H $4.160169-3.367983-1.369523$ H $2.700336-5.556060-0.089207$ H $2.602191-4.2596611 .131284$ $\begin{array}{llll}\mathrm{H} & 1.375646 & -5.282907 & -2.241226\end{array}$
H $-0.008748-4.606424-1.377111$ 93 $24 \mathrm{R}=\mathrm{n}-\mathrm{Pr} \mathrm{ET} / \mathrm{pi}$

Ni $0.144659 \quad 0.102772 \quad 0.076519$ $\begin{array}{llll}\text { N } 2.137138 & 0.105380 & 0.190797\end{array}$ $\begin{array}{llll}\text { N } 0.577030 & 2.065153 & 0.109466\end{array}$ $\begin{array}{llll}\text { C } 1.813497 & 2.445874 & 0.282493\end{array}$ $\begin{array}{llll}\text { C } 2.787201 & 1.234617 & 0.155959\end{array}$ $\begin{array}{llll}\text { C } & -0.140343 & -1.834635 & 0.334613 \\ \text { C } & -1.515445 & -2.405531 & 0.639873\end{array}$ $\begin{array}{llll}\text { C }-1.397594 & -3.819225 & 1.233257\end{array}$ $\begin{array}{llll}\text { C } & -1.397594 & -3.819225 & 1.233257\end{array}$ $\begin{array}{llll}\text { H } & 0.344684 & -2.344107 & -0.512688\end{array}$ $\begin{array}{llll}\mathrm{H} & 0.499553 & -1.923938 & 1.220454 \\ \mathrm{H} & -2.131002 & -2.475651 & -0.271338\end{array}$ $\mathrm{H}-2.131002-2.475651-0.271338$ H $-2.050570-1.7584001 .355002$ $\begin{array}{llll}\mathrm{H}-0.852946 & -4.486198 & 0.54675\end{array}$ H $-0.849940 \quad-3.801320 \quad 2.187478$ H $-2.395603 \quad-4.250087 \quad 1.417417$ C $-1.565705 \quad 0.178498 \quad-0.983980$ C $-0.449518-0.019736-1.814353$ H $-0.213236-0.997402-2.240200$ H $0.054250 \quad 0.805968 \quad-2.322888$ H $-2.246288-0.643493-0.773645$ H $-1.9970591 .168900-0.828286$ $\begin{array}{llll}\text { C } 2.705885 & -1.205154 & 0.020903\end{array}$ C $-0.627281 \quad 2.787498 \quad 0.418564$ C $2.191139 \quad 3.963836 \quad 0.472143$ $\begin{array}{llll}\text { C } 4.344816 & 1.340862 & 0.122428\end{array}$ $\begin{array}{llll}\text { C } 5.137689 & 0.085472 & -0.324522\end{array}$ $\begin{array}{llll}\text { C } 4.834154 & 2.389942 & -0.904447\end{array}$ C 4.8182091 .5955751 .587121 $\begin{array}{llll}\text { C } 2.366621 & 4.557224 & -0.951014\end{array}$ $\begin{array}{llll}\text { C } 3.424664 & 4.274537 & 1.350201\end{array}$ C $1.1013794 .815643 \quad 1.174591$ C $-1.085771 \quad 2.601330 \quad 1.743435$ C $-1.397830 \quad 3.462153-0.551902$ $\begin{array}{llll}\text { C }-2.304515 & 3.180820 & 2.096385\end{array}$ $\begin{array}{llll}\text { C }-2.616323 & 4.015125 & -0.133073\end{array}$ C $-3.069432 \quad 3.894330 \quad 1.174272$ B $-0.294242 \quad 1.6618812 .808252$ F 1.0469122 .0752063 .036405 F $-0.983095 \quad 1.560839 \quad 4.028696$ $\begin{array}{llll}-0.983095 & 1.560839 & 4.028696\end{array}$ $\begin{array}{llll}F & -0.238780 & 0.304151 & 2.216573\end{array}$ $\begin{array}{llll}\text { C }-0.981163 & 3.670241 & -2.00285 \\ \text { C }-0.896330 & 5.170035 & -2.348241\end{array}$ C $-0.8963305 .170035-2.348241$ C $-1.9393243 .005274-3.006708$ H $0.0127843 .217696-2.141031$ H $-0.313682 \quad 5.740955-1.611672$ H $-1.905898 \quad 5.610598-2.380498$ H $-0.438515 \quad 5.307500-3.34105$ H $-2.9583753 .409392-2.892761$ н $-1.986548 \quad 1.917583-2.878669$ н $-1.6042883 .215622-4.035206$ H $-2.654307 \quad 3.044646 \quad 3.121516$ H $-4.023052 \quad 4.338092 \quad 1.466131$ H $\quad-3.225195 \quad 4.554407 \quad-0.860882$ C $2.906271-2.0027861 .171151$ C $2.875777-1.691862-1.290472$ $\begin{array}{llll}\text { C } 3.268927 & -3.337571 & 0.976494\end{array}$ C $3.242342-3.040058-1.412855$ $\begin{array}{llll}\text { C } 3.242342 & -3.040058 & -1.412855 \\ \text { C } 3.427552 & -3.869034 & -0.309494\end{array}$ $\begin{array}{llll}\text { C } 3.427552 & -3.869034 & -0.309494 \\ \text { C } 2.838058 & -1.460866 & 2.595758\end{array}$ C $1.769368-2.1314453 .472923$ $\begin{array}{llll}\text { C } 4.205385 & -1.624451 \quad 3.309987\end{array}$ н $2.595777-0.3888322 .551478$ H $0.752914-1.8783493 .147365$ H $1.885830-3.227658 \quad 3.463847$ H $1.872819-1.783268 \quad 4.513410$ H $5.044018-1.271661 \quad 2.690182$ H $4.393052-2.687576 \quad 3.542244$ H $4.205412-1.0621314 .259760$ B $2.793855-0.862854-2.717687$ F $4.048033-1.034986-3.384738$ F $1.764585-1.416467-3.532488$ H $3.436410-3.977420-3.847048$ H $3.436410-3.9774201 .847048$ $\begin{array}{llll}\text { H } 3.704692 & -4.919808 & -0.437521\end{array}$ \begin{tabular}{l}
$3.395545-3.432573-2.420299$ \\
\hline
\end{tabular} $1.5679840 .540837-2.559314$ $\begin{array}{lllll}1.526984 & 5.824464 & 1.302065\end{array}$ H 0.1755614 .9269600 .605468 н 0.8619334 .4119562 .167644 H $2.808763 \quad 5.564504-0.868002$ н $2.9947063 .952004-1.613304$ H $1.390171 \quad 4.656531-1.434008$ H $3.5509725 .368923 \quad 1.341210$ H $3.231283 \quad 3.972178 \quad 2.390113$ H $4.367952 \quad 3.842329 \quad 1.025584$ H $5.7472522 .189789 \quad 1.590622$ H $4.0783052 .094806 \quad 2.220738$ H 5.0360820 .6297992 .060177 \begin{tabular}{l}
$5.9347342 .346856-0.933332$ \\
\hline
\end{tabular} \begin{tabular}{l}
$4.4568342 .106608-1.899671$ \\
\hline
\end{tabular} $6.5017589 .422552-0.699808$ $16.2010990 .364258-0.222465$ $\begin{array}{llll}\mathrm{H} & 4.973855 & -0.793458 & 0.305355 \\ \mathrm{H} & 4.956336 & -0.183329 & -1.373085\end{array}$

94 $24 \mathrm{R}=\mathrm{n}-\mathrm{Pr} \mathrm{AC} / \mathrm{p}$ Ni $0.163863-0.0916230 .067396$ N $2.180622-0.161947 \quad 0.065109$ N $0.511782-2.102075-0.11173$ $\begin{array}{llll}\text { C } 1.746542 & -2.463323 & -0.282911\end{array}$ $\begin{array}{llll}\text { C } 2.730131 & -1.349580 & 0.134761\end{array}$ $\begin{array}{llll}\text { C } 0.111213 & 1.864635 & -0.174263\end{array}$ C $-1.186054 \quad 2.601690-0.374149$ C $-0.889221 \quad 4.022009 \quad-0.895912$ H $0.6838312 .261436 \quad 0.671781$ H $0.713496 \quad 1.849617-1.097120$ Н $-1.734024 \quad 2.699276 \quad 0.572582$ $\mathrm{H}-1.842345 \quad 2.083049-1.091272$ H $-0.395985 \quad 3.988592-1.879266$ H $-0.2254224 .559263-0.201269$ H $-1.826175 \quad 4.592889-0.995309$ C $-0.393737-0.016206 \quad 1.962824$ C $-1.543333-0.334961 \quad 1.202152$ C $-2.705656 \quad 0.483076 \quad 1.134192$ N $-3.719679 \quad 1.066226 \quad 1.119828$ H $0.188787 \quad-0.815267 \quad 2.427271$ Н $-1.804330-1.382428 \quad 1.020130$ H $-0.267093 \quad 0.967012 \quad 2.417786$ C $2.952664 \quad 1.061698-0.004265$ C $-0.668937-2.858773-0.404027$ C $2.217133-3.734186-1.057389$ C $4.101292-1.6794390 .811723$ $\begin{array}{llll}\text { C } 3.698314 & -2.494487 & 2.071768\end{array}$ $\begin{array}{lll}\text { C } 4.880187 & -0.480233 & 1.380348\end{array}$ $\begin{array}{lll}\text { C } 5.171367-2.400566 & -0.042655\end{array}$ $\begin{array}{lll}\text { C } 3.116110 & -3.234648 & -2.222221\end{array}$ $\begin{array}{llll}\text { C } 2.913353 & -4.795190 & -0.170068\end{array}$ $\begin{array}{llll}\text { C } 1.100088 & -4.530372 & -1.768504\end{array}$ $\begin{array}{llll}\text { C }-1.381761 & -2.507891 & -1.571084\end{array}$ $\begin{array}{llll}\text { C }-1.177962 & -3.763897 & 0.549576\end{array}$ C $-2.602885-3.146882-1.790118$ $\begin{array}{llll}\text { C }-2.412055 & -4.367191 & 0.271885\end{array}$ $\begin{array}{llll}\text { C }-3.119960 & -4.074996 & -0.886398\end{array}$ B $-0.840327-1.417461-2.649744$ F $-0.513448-0.160734-1.937465$ F $0.364496-1.833797-3.280268$ F $-1.811491-1.114225-3.611694$ $\begin{array}{llll}\text { F } & -1.811491 & -1.114225 & -3.611694 \\ \text { C }-0.479586 & -4.088657 & 1.859572\end{array}$ $\begin{array}{llll}\text { C }-0.479586 & -4.088657 & 1.859572\end{array}$ $\begin{array}{llll}C-0.227965 & -5.621754 & 2.023285 \\ C & -1.269317 & -3.576715 & 3.074755\end{array}$ C $-1.269317-3.5767153 .074755$ H $0.500756-3.5904391 .863961$ H $0.289519-6.0457791 .148971$ H $0.381240-5.808634 \quad 2.923699$ H $-1.183915 \quad-6.158572 \quad 2.141967$ H $-1.425977-2.490185 \quad 3.034079$ H $-0.730824-3.811418 \quad 4.006808$ H $-2.260138-4.0564493 .121849$ H $-3.158571-2.885565-2.692328$ H $-4.082449-4.553772-1.073619$ H $-2.828412 \quad-5.074146 \quad 0.991570$ C $3.430924 \quad 1.379182-1.300310$ C $3.098054 \quad 1.943422 \quad 1.090644$ $\begin{array}{llll}\text { C } 4.155910 & 2.565240 & -1.460637\end{array}$ C $3.834327 \quad 3.112650 \quad 0.850898$ $\begin{array}{lllll}\text { C } 4.375562 & 3.424047 & -0.393844\end{array}$ C $3.1508320 .546573-2.547920$ C $2.243444 \quad 1.291590-3.536001$ $\begin{array}{llll}\text { C } 4.434749 & 0.123139 & -3.275777\end{array}$ H $2.609384-0.362473-2.262075$ H $1.2788291 .550881-3.080272$ H $2.031035 \quad 0.647974 \quad-4.403745$ $\begin{array}{lllll}\text { H } & 2.723457 & 2.218010 & -3.891607\end{array}$ H $5.139426-0.393507-2.603542$ H $4.188639-0.555168-4.110965$ $\begin{array}{lllll}\text { H } & 4.961183 & 0.997487 & -3.697324\end{array}$ B $2.491644 \quad 1.806613 \quad 2.624565$ F 1.2072852 .3860842 .680559 F $3.2328202 .573243 \quad 3.541091$ F 2.4239950 .4410373 .054237 H $4.5399952 .818376-2.451506$ $\begin{array}{lllll}\text { H } & 4.949894 & 4.344070 & -0.537127\end{array}$ $\begin{array}{lllll}\mathrm{H} & 4.949894 & 4.344070 & -0.537127 \\ \mathrm{H} & 3.984274 & 3.789908 & 1.695521\end{array}$ H $5.758210-0.8994701 .903590$ H 5.2509320 .2003750 .599180 H $4.296032 \quad 0.081464 \quad 2.114500$ н $4.613346-2.8653302 .563949$ H $3.174702-1.8229462 .769846$ $\begin{array}{llll}\text { H } 3.049284 & -3.353879 & 1.865344\end{array}$ H $6.028326-2.6072270 .622176$ H $4.884727-3.344361-0.508291$ H $5.533946-1.724319-0.834987$ H $3.703998-4.082891-2.616472$ H $2.464549-2.852307-3.02351$ H $3.808957-2.432121-1.955192$ H $3.207532-5.635661-0.823429$ H $3.803201-4.4639150 .370842$ $\begin{array}{llll}3.803201 & -4.463915 & 0.370842\end{array}$ $\begin{array}{llll}4 & 2.198763 & -5.189746 & 0.570336\end{array}$ $\begin{array}{llll}\mathrm{H} & 1.612093 & -5.306236 & -2.362989\end{array}$ $\begin{array}{llll}\text { H } & 0.415495 & -5.038778 & -1.07782\end{array}$ 94 
$24 \mathrm{R}=\mathrm{n}-\mathrm{Pr} \quad \mathrm{AC} / \mathrm{N}$

Ni $0.021208-0.045149 \quad 0.012917$ N $1.941214-0.044369-0.154708$ N $0.373038-1.942375-0.269670$ $\begin{array}{llll}\text { C } 1.594057 & -2.364852 & -0.453860\end{array}$ $\begin{array}{llll}\text { C } 2.583441 & -1.183668 & -0.250660\end{array}$ $\begin{array}{lllll}\text { C }-0.201916 & 1.943790 & 0.135011\end{array}$ $\begin{array}{llll}\text { C } & -1.517248 & 2.679798 & -0.049965\end{array}$ C $-2.1352252 .559746-1.443304$ $\begin{array}{llll}\text { H } & 0.529556 & 2.288483 & -0.608489\end{array}$ $\begin{array}{llll}\mathrm{H} & 0.529556 & 2.288483 & -0.608489\end{array}$ $\begin{array}{llll}H & 0.198345 & 2.075082 & 1.152148\end{array}$ $\mathrm{H}-1.301925 \quad 3.749624 \quad 0.152034$ $\mathrm{H}-2.2490692 .3867800 .721140$ $\mathrm{H}-2.3050831 .507905-1.71763$ $\mathrm{H}-1.4589902 .981674-2.203202$ H $-3.092984 \quad 3.102142-1.505605$ $\begin{array}{llll}\mathrm{N} & -1.712064 & -0.116904 & 0.401133\end{array}$ C $-2.841380-0.1683220 .690342$ $\begin{array}{llll}\text { C }-4.184477 & -0.139638 & 1.109693\end{array}$ $\begin{array}{llll}\text { C } & -5.139705 & -0.953064 & 0.62813\end{array}$ H $-4.415644 \quad 0.610184 \quad 1.872994$ $\begin{array}{lllll}\text { H } & -6.161752 & -0.873427 & 0.996884\end{array}$ $\begin{array}{llll}\text { C } & -1.119978 & -4.836343 & 2.448217\end{array}$ C $2.584136 \quad 1.235158-0.008010$ C $-0.849590-2.645611-0.541071$ C $1.929680-3.878042-0.727473$ C $1.929680-3.878042-0.727473$ $\begin{array}{llll}C & 4.136562 & -1.310442 & -0.220489\end{array}$ $\begin{array}{llll}\text { C } 4.658376 & -1.492926 & -1.704283\end{array}$ $\begin{array}{llll}C & 4.969451 & -0.113844 & 0.307207\end{array}$ C $4.569275-2.4356450 .749812$ $\begin{array}{lll}C & 2.102316-4.577437 & 0.652039\end{array}$ $\begin{array}{llll}C & 3.149506 & -4.134417 & -1.640928\end{array}$ C $0.823234-4.677273-1.466585$ $\begin{array}{llll}\text { C }-1.362524 & -2.513123 & -1.849043\end{array}$ $\begin{array}{llll}\text { C }-1.553410 & -3.269572 & 0.506249\end{array}$ $\begin{array}{llll}\text { C }-2.605489 & -3.105500 & -2.093665\end{array}$ $\begin{array}{llll}\text { C }-2.796662 & -3.833698 & 0.193666\end{array}$ $\begin{array}{llll}\text { C }-3.319718 & -3.766871 & -1.094457\end{array}$ B $-0.613661-1.682296-3.056669$ F $-1.306481-1.890378-4.276924$ F $-0.622839-0.288712-2.736335$ F $0.752122-2.095731-3.221789$ C $0.752122-2.095731-3.221789$ C $-1.008471-3.394354 \quad 1.923284$ $\begin{array}{llll}C & -1.689728 & -2.438845 & 2.916960\end{array}$ H $0.054919-3.1161621 .902212$ H $-0.744604-5.573354 \quad 1.722233$ $\mathrm{H}-0.549371-4.9430013 .384350$ $\begin{array}{lllll}\mathrm{H} & -2.168327 & -5.094783 & 2.668359\end{array}$ H $-1.433389-1.392712 \quad 2.705687$ H $-1.350053 \quad-2.662463 \quad 3.941356$ H $-2.785602-2.5589462 .881734$ H $-3.004003-3.036854-3.107921$ H $-4.282407-4.233310-1.317418$ H $-3.358641-4.347118 \quad 0.976272$ C $2.899110 \quad 1.962638-1.174980$ C $2.724683 \quad 1.752361 \quad 1.294359$ C $3.4388953 .240643-1.004498$ C $3.2745083 .036381 \quad 1.393410$ $\begin{array}{llll}\text { C } 3.638245 & 3.777468 & 0.266741\end{array}$ C $2.676461 \quad 1.436369-2.586176$ $\begin{array}{llll}\text { C } & 2.676461 & 1.436369 & -2.586176 \\ \text { C } & 1.465951 & 2.093736 & -3.265299\end{array}$ $\begin{array}{llll}\text { C } & 1.465951 & 2.093736 & -3.265299 \\ \text { C } 3.927235 & 1.644606 & -3.503136\end{array}$ $\begin{array}{llll}\text { H } 2.469585 & 0.357936 & -2.539651\end{array}$ $\begin{array}{lllll}\text { H } & 2.469585 & 0.357936 & -2.539651 \\ \text { H } & 0.530432 & 1.825267 & -2.761748\end{array}$ $\begin{array}{lllll}\mathrm{H} & 0.530432 & 1.825267 & -2.761748 \\ \mathrm{H} & 1.578351 & 3.190881 & -3.274153\end{array}$ $\begin{array}{lllll}\mathrm{H} & 1.578351 & 3.190881 & -3.274153 \\ \mathrm{H} & 1.382182 & 1.737916 & -4.304425\end{array}$ $\begin{array}{lllll}\mathrm{H} & 1.382182 & 1.737916 & -4.304425 \\ \mathrm{H} & 4.861729 & 1.323884 & -3.015362\end{array}$ $\begin{array}{lllll}\text { H } & 4.861729 & 1.323884 & -3.015362 \\ \text { H } & 4.037376 & 2.707951 & -3.777262\end{array}$ $\begin{array}{llll}\text { H } 3.796810 & 1.068159 & -4.434847\end{array}$ $\begin{array}{llll}\text { B } 2.335944 & 0.948693 & 2.683417\end{array}$ F $2.2701351 .878556 \quad 3.754328$ F $3.337164-0.0354712 .981595$ F $1.077067 \quad 0.277306 \quad 2.570201$ H $3.705851 \quad 3.827158-1.886345$ H $4.063708 \quad 4.779986 \quad 0.374920$

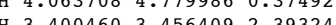
$\begin{array}{llll}\text { H } 3.4004603 .456409 & 2.393248\end{array}$ $\begin{array}{llll}\text { H } & -4.908865 & -1.701034 & -0.1305 \\ \text { H } 6.022094 & -0.445935 & 0.245248\end{array}$ $\begin{array}{llll}\text { H } 6.022094 & -0.445935 & 0.245248\end{array}$ $\begin{array}{lll}4.882935 & 0.796352-0.296298\end{array}$ $\begin{array}{llll}\text { H } & 4.739808 & 0.115173 & 1.357114 \\ \mathrm{H} & 5.561562 & -2.128068 & -1.70518\end{array}$ H $5.561562-2.128068-1.705188$ H $3.921440-1.920376-2.393920$ H $4.935443-0.504838-2.096012$ $\begin{array}{llll}\text { H } 5.670725 & -2.462720 & 0.769147\end{array}$ H $4.221993-2.175107 \quad 1.763251$ H $4.222519-3.436306 \quad 0.501037$ H $3.252858-5.226650-1.738715$ H $2.951943-3.728408-2.643960$ H $4.103550-3.753719-1.282059$ H $1.230804-5.689915-1.622844$ H $-0.105082-4.788178-0.899964$ H $0.600977-4.232410-2.444742$ $\begin{array}{llll}\mathrm{H} & 0.600977 & -4.232410 & -2.444742\end{array}$ $\begin{array}{llll}\text { H } 2.671251 & -5.512360 & 0.513757\end{array}$ $\begin{array}{llll}\text { H } & 2.612532 & -3.969992 & 1.408519 \\ \text { H } & 1.117840 & -4.843245 & 1.054115\end{array}$ 94

$24 \mathrm{R}=\mathrm{CHCNE} \mathrm{ET} / \mathrm{pi}$
Ni $0.143172 \quad 0.157266 \quad 0.063634$ $\begin{array}{llll}\text { N } 2.154915 & 0.158179 & 0.146633\end{array}$ N $0.5826512 .121779 \quad 0.172559$ C $1.825576 \quad 2.488391 \quad 0.330036$ C $2.797970 \quad 1.289502 \quad 0.090686$ $\begin{array}{llll}C & -0.252961 & -1.848051 & 0.190751\end{array}$ C $-1.091357-2.211785 \quad 1.430620$ C $-1.198704 \quad-3.731559 \quad 1.618516$ C $-0.854047-2.459849-0.964908$ N $-1.443346-2.985714-1.828438$ $\begin{array}{llll}\mathrm{N} & -1.443346 & -2.985714 & -1.828438\end{array}$ $\begin{array}{llll}\text { H } & 0.774889 & -2.229655 & 0.287310\end{array}$ $\begin{array}{llll}\mathrm{H}-2.097726 & -1.770634 & 1.34801\end{array}$ H $-0.625905-1.77119620 .316552$ $\mathrm{H}-1.742757-4.2001600 .784468$ H $-0.196774-4.187683 \quad 1.666079$ H $-1.727415-3.9606982 .557206$ $\begin{array}{llll}c & -1.618752 & 0.320694 & -0.903495\end{array}$ $\begin{array}{llll}\text { C }-0.539878 & 0.282162 & -1.805103\end{array}$ H $-0.279459-0.619156-2.365421$ н $-0.097714 \quad 1.194774 \quad-2.208310$ H $-2.257985-0.557185-0.789135$ H $-2.072313 \quad 1.264233-0.589567$ C $2.748694-1.151601 \quad 0.053668$ $\begin{array}{lllll}\text { C } & -0.593432 & 2.876811 & 0.529090\end{array}$ C $2.217089 \quad 3.974873 \quad 0.679820$ $\begin{array}{llll}\text { C } 4.338099 & 1.409160 & -0.174533\end{array}$ $\begin{array}{llll}\text { C } 5.018428 & 0.205657 & -0.876705\end{array}$ C $4.6244822 .553299-1.173318$ $\begin{array}{llll}\text { C } & 4.624482 & 2.553299 & -1.173318 \\ \text { C } 5.110476 & 1.550467 & 1.171640\end{array}$ C $2.280004 \quad 4.775371-0.643615$ $\begin{array}{llll}\text { C } 3.527289 & 4.144564 & 1.469599\end{array}$ C $1.217316 \quad 4.7326331 .595813$ $\begin{array}{llll}\text { C }-1.045730 & 2.651137 & 1.849891\end{array}$ $\begin{array}{llll}\text { C }-1.334272 & 3.645449 & -0.394716\end{array}$ $\begin{array}{lll}\text { C }-2.230131 & 3.268995 & 2.248737\end{array}$ $\begin{array}{llll}\text { C }-2.517156 & 4.238076 & 0.072457\end{array}$ $\begin{array}{llll}\text { C }-2.965387 & 4.068521 & 1.374977\end{array}$ B $-0.271618 \quad 1.655317 \quad 2.872617$ F $1.062717 \quad 2.048963 \quad 3.160034$ F $-0.989463 \quad 1.470780 \quad 4.063994$ $\begin{array}{llll}\text { F }-0.183372 & 0.343345 & 2.196031\end{array}$ C $-0.949491 \quad 3.906852-1.846311$ C $-0.8789325 .419934-2.152833$ $\begin{array}{llll}C & -0.878932 & 5.419934 & -2.152833\end{array}$

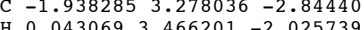
H $0.0430693 .466201-2.025739$ $\mathrm{H}-0.3354325 .987080-1.383664$ $\mathrm{H}-1.8943875 .844174-2.214436$ $\mathrm{H}-0.3892355 .586701-3.126081$ $\mathrm{H}-2.944425 \quad 3.705077-2.703628$ H $-2.0127832 .190439-2.733918$ H $-1.617265 \quad 3.498702 \quad-3.875245$ H $-2.5754103 .098216 \quad 3.270062$ H $-3.892127 \quad 4.544618 \quad 1.700315$ H $-3.102717 \quad 4.848931-0.616472$ C $3.106596-1.796794 \quad 1.260645$ $\begin{array}{llll}\text { C } 2.781730 & -1.792754 & -1.201541\end{array}$ $\begin{array}{llll}\text { C } 3.465639 & -3.147458 & 1.183566\end{array}$ C $3.162762-3.140545-1.204068$ C $3.482041-3.828236-0.036197$ $\begin{array}{llll}\text { C } 3.482041 & -3.828236 & -0.036197 \\ \text { C } 3.150704 & -1.108803 & 2.618363\end{array}$ $\begin{array}{llll}C & 3.150704 & -1.108803 & 2.618363\end{array}$ $\begin{array}{llll}\text { C } 2.049458 & -1.588545 & 3.571460\end{array}$ $\begin{array}{llll}\text { C } 4.529201 & -1.314227 & 3.322119\end{array}$ H $3.002198-0.0297402 .473967$ $1.058021-1.3197123 .193894$ H $2.099657-2.681215 \quad 3.707973$ H $2.167891-1.1075534 .555596$ H $5.375248-1.1237312 .643588$ H $4.624163-2.348983 \quad 3.693755$ H $4.608275-0.636097 \quad 4.189204$ B $2.525247-1.124991-2.693291$ F $3.666547-1.429024-3.496886$ F $1.376335-1.698418-3.29429$ H $3.743231-3.6756732 .099675$ H $3.755512-4.887175-0.066677$ H $3.208182-3.652014-2.167582$ F $2.3783200 .299732-2.645191$

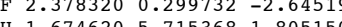
$\begin{array}{llll}\mathrm{H} & 1.674620 & 5.715368 & 1.805150\end{array}$ 1.070922 .9265211 .137565 1.0709224 .2039242 .548373 H $2.8101895 .727342-0.461269$ H $2.779585 \quad 4.251863-1.467562$ H $1.2658195 .017374-0.974902$ H $3.659010 \quad 5.2246051 .650213$ H 3.4432383 .6551872 .45359 H $4.4315763 .793278 \quad 0.973089$ H 5.9970002 .1935491 .032574 H $4.517667 \quad 1.9502812 .000927$ $\begin{array}{llll}\text { H } & 5.464905 & 0.558459 & 1.481453\end{array}$ H $5.6934892 .514965-1.435613$ H $4.0488612 .375081-2.096029$ H $4.4183833 .558406-0.808166$ $6.0773620 .496105-0.987188$ H $6.0773620 .496105-0.987188$ $\begin{array}{llll}\text { H } & 5.000678 & -0.716738 & -0.289295 \\ \text { H } & 4.613419 & 0.010549 & -1.875723\end{array}$ 95

24

HCNEt $\mathrm{AC} / \mathrm{p}$

Ni $0.149675-0.1586950 .021483$

N $2.217263 \quad-0.1959360 .031449$ N $0.556272-2.158373-0.138930$ $\begin{array}{llll}\text { C } 1.798349 & -2.504352 & -0.276900\end{array}$ C $2.765881-1.3763770 .144755$ C $-0.083831 \quad 1.865095-0.113650$ C $-0.384131 \quad 2.288291-1.552610$ C $-0.314000 \quad 3.816110-1.706857$ C $-0.990446 \quad 2.513976 \quad 0.793848$ N $-1.733743 \quad 3.104425 \quad 1.476790$ H $0.9355762 .145751 \quad 0.193726$ H $-1.355762601 .920317-1.859468$ $\mathrm{H}-1.3752601 .920317-1.859468$
$\mathrm{H}-0.354898$ $\begin{array}{llll}\mathrm{H} & 0.354898 & 1.824534 & -2.213366\end{array}$ H $0.6666064 .196842-1.378961$ $\mathrm{H}-1.0916254 .308800-1.104049$ H $-0.460714 \quad 4.091796-2.762913$ $\begin{array}{lllll}C & -0.555373 & -0.156241 & 1.874599\end{array}$ $\begin{array}{llll}C & -1.616203 & -0.487264 & 0.995353\end{array}$ $\begin{array}{llll}\text { C }-2.781732 & 0.279889 & 0.702663\end{array}$ $\begin{array}{lllll}\mathrm{N} & -3.813864 & 0.759451 & 0.434825\end{array}$ н $-1.827433-1.5449710 .811076$ H $-0.009532-0.9653512 .365079$ $\begin{array}{lllll}\text { H } & -0.473826 & 0.814796 & 2.362759\end{array}$ C $2.984962 \quad 1.031129-0.007880$ C $-0.597186-2.954933-0.457947$ $\begin{array}{llll}\text { C } 2.306045 & -3.780401 & -1.015684\end{array}$ $\begin{array}{llll}\text { C } 4.106767 & -1.700957 & 0.880927\end{array}$ $\begin{array}{llll}\text { C } 3.628250 & -2.459013 & 2.148741\end{array}$ C $3.628250-2.4590132 .148741$ $\begin{array}{llll}C & 4.889782 & -0.493687 & 1.435818\end{array}$ $\begin{array}{llll}\text { C } 3.210654 & -3.270730 & -2.174986\end{array}$ C $3.210654-3.270730-2.174986$ C $2.989658-4.816790-0.087891$ $\begin{array}{llll}\text { C } 1.233556 & -4.627052 & -1.737671\end{array}$ $\begin{array}{llll}\text { C }-1.283403 & -2.634919 & -1.648122\end{array}$ $\begin{array}{llll}C & -1.106640 & -3.864393 & 0.491187\end{array}$ $\begin{array}{llll}\text { C }-2.497855 & -3.282833 & -1.879621\end{array}$ $\begin{array}{llll}\text { C }-2.331284 & -4.480008 & 0.198642\end{array}$ $\begin{array}{llll}\text { C }-3.027259 & -4.197754 & -0.969842\end{array}$ B $-0.714311-1.587542-2.753707$ F $-0.176997-0.394849-2.060196$ F $0.380427-2.127221-3.485119$ F $-1.717116-1.161655-3.630163$ $\begin{array}{llll}C & -0.416519 & -4.193325 & 1.806591\end{array}$ C $-0.161030-5.706031 \quad 1.955718$ $\begin{array}{llll}C & -0.161030 & -5.706031 & 1.955718\end{array}$ $\begin{array}{llll}C & -1.218477 & -3.690731 & 3.017945\end{array}$ H $0.558432-3.6843561 .820659$ $0.351693-6.128602-1.077273$ $\begin{array}{llll}\text { H } & 0.454143 & -5.903065 & 2.850758 \\ \mathrm{H} & -1.112185 & -6.252423 & 2.077442\end{array}$ $\begin{array}{llll}\text { H } & -1.112185 & -6.252423 & 2.077442 \\ \text { H } & -1.396965 & -2.608078 & 2.972383\end{array}$ $\begin{array}{llll}\mathrm{H} & -1.396965 & -2.608078 & 2.972383 \\ \mathrm{H} & -0.676646 & -3.911275 & 3.951532\end{array}$ H $-2.199820-4.189403 \quad 3.065445$ $\mathrm{H}-3.041172-3.035981-2.793282$ H $-3.987887-4.678044-1.162327$ H $-2.752398 \quad-5.186406 \quad 0.915954$ C $3.5459191 .356713-1.268588$ C $3.063345 \quad 1.9020881 .102532$ C $4.252822 \quad 2.560334-1.380176$ C $3.7857113 .088799 \quad 0.912359$ C $4.3842753 .421299-0.300328$ $\begin{array}{llll}\text { C } 4.384275 & 3.421299 & -0.300328 \\ \text { C } 3.438649 & 0.485743 & -2.515255\end{array}$ $\begin{array}{llll}\text { C } 3.438649 & 0.485743 & -2.515255 \\ \text { C } 2.7893992 & 1.214430 & -3.698682\end{array}$ $\begin{array}{llll}\text { C } & 2.789392 & 1.214430 & -3.698682 \\ \text { C } & 4.827288 & -0.035471 & -2.964619\end{array}$ $\begin{array}{llll}\text { C } & 4.827288 & -0.035471 & -2.964619 \\ \text { H } & 2.801205 & -0.378695 & -2.293787\end{array}$ H $1.785084 \quad 1.576765-3.446056$ H $2.691108 \quad 0.524411-4.551093$ H $3.3955552 .076078-4.020413$ H $5.362662-0.537502-2.144047$ H $4.715588-0.747916-3.80052$ $\begin{array}{llll}\text { H } & 5.461822 & 0.799948 & -3.309851\end{array}$ B $2.384444 \quad 1.710553 \quad 2.599782$ F $1.0835412 .223661 \quad 2.584585$ F 3.0565162 .4777873 .567628 F 2.3509090 .3296712 .995512 H $4.703297 \quad 2.819978-2.341046$ H $4.9445314 .354894-0.406976$ 4.944531
4 $\begin{array}{lllll}\text { H } 3.876531 & 3.759962 & 1.770274\end{array}$ $\begin{array}{lllll}\text { H } & 5.758108 & -0.909850 & 1.977567\end{array}$ $\begin{array}{llll}\text { H } 5.275994 & 0.168119 & 0.645806\end{array}$ $\begin{array}{llll}\mathrm{H} & 5.963318 & -2.775518 & 0.852719\end{array}$ $\begin{array}{cccc}\text { H } & 5.963318 & -2.775518 & 0.852719 \\ \text { H } & 4.883142 & -3.409096 & -0.396681\end{array}$ $\begin{array}{llll}\text { H } & 4.883142 & -3.409096 & -0.396681 \\ \text { H } 5.678622 & -1.845142 & -0.626805\end{array}$ $\begin{array}{llll}\text { H } & 5.678622 & -1.845142 & -0.62680 \\ \text { H } & 4.513676 & -2.811012 & 2.704451\end{array}$ $\begin{array}{llll}\text { H } & 4.513676 & -2.811012 & 2.704451 \\ \text { H } 3.068223 & -1.760141 & 2.788206\end{array}$ $\begin{array}{llll}\text { H } 3.068223 & -1.760141 & 2.788206 \\ \text { H } 2.990441 & -3.327071 & 1.940878\end{array}$ H $3.784200-4.121620-2.584829$ н $2.558761-2.869873-2.968286$ H $3.917811-2.484129-1.895577$ $\begin{array}{llll}\text { H } 3.388217 & -5.624434 & -0.727284\end{array}$ 
N $1.017145-0.193505-0.793418$ N $-1.426494-0.013293-0.623353$ C $-1.0363420 .110400-1.869468$ $\begin{array}{llll}\text { C } 0.508110 & -0.023859 & -1.979487\end{array}$ $\begin{array}{llll}\text { C } 2.404481 & -0.357589 & -0.450545\end{array}$ $\begin{array}{llll}\text { C } & -2.767039 & 0.220515 & -0.146085\end{array}$ $\begin{array}{llll}\text { C } & -2.016551 & 0.211461 & -3.080847\end{array}$ $\begin{array}{llll}\text { C } & 1.367646 & 0.187736 & -3.259307\end{array}$ C $1.384519 \quad 1.755828-3.535955$ c $0.906069-0.642444-4.480824$ C $2.860582-0.214138-3.171144$ C $2.860582-0.214138-3.171144$ $\begin{array}{llll}C & -1.633044 & 1.301437 & -4.117207\end{array}$ $\begin{array}{llll}\text { C } & -2.075167 & -1.225065 & -3.688060 \\ C & -3.488126 & 0.595792 & -2.784576\end{array}$ $\begin{array}{llll}C & -3.071735 & 1.543813 & 0.224636\end{array}$ $\begin{array}{llll}C & -3.630416 & -0.871674 & 0.070521\end{array}$ $\begin{array}{llll}\text { C }-4.320580 & 1.744394 & 0.826641\end{array}$ $\begin{array}{llll}\text { C }-4.859986 & -0.598830 & 0.678953\end{array}$ C $-5.206488 \quad 0.694226 \quad 1.060087$ $\begin{array}{llll}\text { B }-2.082072 & 2.830664 & -0.049932\end{array}$ $\begin{array}{lllll}\text { F } & -2.614238 & 3.981009 & 0.581691\end{array}$ F $-0.763187 \quad 2.584024 \quad 0.451570$ F $-1.9718773 .072206-1.459996$ $\begin{array}{llll}\text { C }-3.302422 & -2.293290 & -0.366327\end{array}$ $\begin{array}{llll}\text { C }-4.429897 & -2.893228 & -1.225344\end{array}$ C $-3.012029-3.2293960 .815931$ H $-2.387630-2.261086-0.976410$ $\mathrm{H}-2.387630-2.261086-0.976410$ $\mathrm{H}-4.735833-2.224029-2.043361$ $\mathrm{H}-4.099535-3.849028-1.661851$ H $-5.322997-3.098563-0.613517$ H $-2.101136-2.942948 \quad 1.355491$ H $-2.859874-4.2561550 .446828$ H $-3.853752-3.235594 \quad 1.527216$ $\begin{array}{llll}\text { H } & -4.582064 & 2.763534 & 1.117831\end{array}$ H $-6.1690790 .879008 \quad 1.541293$ H $-5.557526-1.419216 \quad 0.857230$ $\begin{array}{llll}\text { C } 3.131808 & 0.764758 & -0.015784\end{array}$ $\begin{array}{llll}\text { C } 2.890277 & -1.678268 & -0.404430\end{array}$ $\begin{array}{llll}\text { C } 4.450561 & 0.546071 & 0.402344\end{array}$ $\begin{array}{llll}\text { C } 4.209069 & -1.834820 & 0.028398\end{array}$ $\begin{array}{llll}\text { C } 5.001836 & -0.732771 & 0.416614\end{array}$ C $2.5469332 .167249 \quad 0.026130$

C $2.308867 \quad 2.6328691 .469693$ $\begin{array}{llll}\text { C } & 3.426252 & 3.203198 & -0.703995\end{array}$ \begin{tabular}{llll}
$C$ & 2.426252 & 3.203198 & -0.703995 \\
\hline & 1.562371 & 2.154006 & -0.463405
\end{tabular} $\begin{array}{lll}\mathrm{C} & 1.5623712 .154006-0.463405\end{array}$ $\begin{array}{ll}1.645282 & 1.9407552 .005026\end{array}$ 3.2635542 .7005782 .018288 H $1.826103 \quad 3.622198 \quad 1.469907$ H $3.7047402 .871413-1.716446$ H $4.360156 \quad 3.394420-0.148031$ H $2.881957 \quad 4.158554-0.787586$ B $1.976951-2.987973-0.809940$ F $2.704008-4.173119-0.539560$ F $1.646288-2.946051-2.209906$ F $0.755299-2.986722-0.070376$ H $5.053466 \quad 1.400216 \quad 0.718499$ H $6.039490-0.8800410 .734303$ H $4.617310-2.847013 \quad 0.064354$ H $3.2909730 .021097-4.159604$

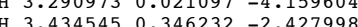

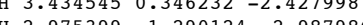
$\begin{array}{llll}H & 2.975399 & -1.290124 & -2.987986\end{array}$ $\begin{array}{lllll}\mathrm{H} & 1.519889 & 1.934718 & -4.617482\end{array}$ $10.4847452 .285492-3.194800$ H $2.2422122 .192343-3.003842$ H $1.585963-0.402925-5.316235$ H $1.026141-1.712796-4.243222$ H $-0.111436-0.473647-4.831517$ H $-2.382341 \quad 1.254994 \quad-4.92623$ $\mathrm{H}-1.709697 \quad 2.289742-3.635959$ H $-0.652553 \quad 1.208312-4.579642$ H $-2.354264-1.161644-4.752958$ H $-1.141174-1.792948-3.604460$ H $-2.851227-1.803536-3.169361$ H $-3.9984590 .588539-3.762332$ $\mathrm{H}-4.018352-0$. H $-3.5646321 .605585-2.362059$ $\mathrm{C}-3.564632-1.605585-2.362059$ C $-1.333594-0.4044372 .384211$ C -1.1543500 .9100693 .166190$ C $-2.1975891 .068169 \quad 4.278292$ C $-1.111221-1.541936-3.238292$ $\mathrm{N}-0.902763-2.425650 \quad 3.978679$ H $-2.353020-0.472925 \quad 1.97925$ $\begin{array}{lllll}\text { H } & -0.143380 & 0.937433 & 3.604356\end{array}$ H $-1.227546 \quad 1.747895 \quad 2.458936$ $\begin{array}{llll}\text { H } & -2.039061 & 2.013059 & 4.821568\end{array}$ $\mathrm{H}-3.2139101 .084515 \quad 3.854010$ H $-2.137624 \quad 0.236811 \quad 4.998988$ N $1.142214 \quad-0.772376 \quad 1.950189$ C $1.856212-1.174692 \quad 2.775142$ C $2.588042-1.710474 \quad 3.850930$ C $3.909948-1.5406853 .999978$ H $1.979558-2.281088 \quad 4.560084$ H $1.926934-1.973518 \quad 4.850100$

$\begin{array}{llll}\mathrm{H} & 4.426934 & -1.973518 & 4.858100 \\ \mathrm{H} & 4.491616 & -0.978106 & 3.266000\end{array}$ 
73

Ni $0.0084650 .105564-0.153676$ $\begin{array}{lllll}\mathrm{N} & 1.839369 & 0.030489 & -0.079479\end{array}$ N $0.277000 \quad 1.980007 \quad 0.089579$ $\begin{array}{llll}\text { C } 2.440224 & 1.209650 & -0.017412\end{array}$ $\begin{array}{lllll}\text { C } 1.534857 & 2.348996 & 0.024819\end{array}$ $\begin{array}{llll}\text { C } 2.563595 & -1.157844 & 0.254637\end{array}$ $\begin{array}{lllll}\text { C }-0.793348 & 2.828247 & 0.449146\end{array}$ C $3.9211141 .386367 \quad 0.006568$ C 3.9211141 .3863670 .006568 C $2.0238813 .756529-0.000949$ $\begin{array}{llll}C & -0.443355 & -1.697673 & -0.452428\end{array}$ $\begin{array}{lllll}\mathrm{H} & -1.440507 & -1.696492 & 0.047018\end{array}$ H $0.191972-2.475723-0.008966$ H $-0.555782-1.859018-1.538825$ B $0.7065930 .156250 \quad 5.414579$ F $1.840434 \quad-0.538938 \quad 5.903436$ F $0.146516 \quad 1.040696 \quad 6.443872$ F $-0.281540 \quad-0.752318 \quad 4.965939$ C $1.1882501 .168951 \quad 4.158257$ C $-0.047063 \quad 1.785287 \quad 3.499350$ C $2.149951 \quad 0.489126 \quad 3.182536$ H $1.7470151 .966692 \quad 4.682486$ $\begin{array}{llllll}\text { H } & 4.428955 & 0.467644 & 0.325353\end{array}$ H $4.2092562 .204567 \quad 0.680906$ H $4.278099 \quad 1.648883-1.005874$ $\begin{array}{lllll}\text { H } & 2.640977 & 3.995707 & 0.879901\end{array}$ $\begin{array}{lllll}\text { H } & 2.640977 & 3.995707 & 0.879901 \\ \text { H } 2.656074 & 3.915048 & -0.890373\end{array}$ $\begin{array}{lllll}\text { H } & 2.656074 & 3.915048 & -0.890373 \\ \text { H } & 1.181993 & 4.460911 & -0.027899\end{array}$ $\begin{array}{llll}\text { H } & 1.181993 & 4.460911 & -0.027899\end{array}$ $\begin{array}{llll}\text { C }-1.909126 & 4.206898 & 2.070102\end{array}$ $\begin{array}{llll}\text { C }-2.981421 & 4.354111 & 1.198919\end{array}$ $\begin{array}{llll}C & -2.981421 & 4.354111 & 1.198919 \\ C & -2.985129 & 3.694512 & -0.030966\end{array}$ $\begin{array}{llll}\text { C } & -2.985129 & 3.694512 & -0.030966 \\ \text { C }-1.905211 & 2.904837 & -0.418191\end{array}$ $\begin{array}{llll}\text { C }-1.905211 & 2.904837 & -0.418191 \\ \text { C }-1.880877 & 2.174364 & -1.748852\end{array}$ C $-1.1108362 .974119-2.808650$ C $-3.267234 \quad 1.780195 \quad-2.257126$ H $-1.306488 \quad 1.226886-1.593246$ H $-0.083802 \quad 3.190397-2.481202$ H $-1.619076 \quad 3.934328-2.993966$ H $-1.063758 \quad 2.417019-3.756886$ $\mathrm{H}-3.845620 \quad 1.250258-1.486548$ H $-3.8400722 .665287-2.5873522$ H $-3.172245 \quad 1.124561-3.573522$ $\begin{array}{llll}\mathrm{H}-3.172245 & 1.124561 & -3.13513\end{array}$ H $-3.849490-3.791712-0.686387$ 1.8365444 .9660381 .487244 C -1.9361904 .6802373 .052894$ C $2.504902-1.5477141 .608826$ $\begin{array}{llll}\text { C } 3.237533 & -2.673767 & 1.987309\end{array}$ C $3.979716-3.396222 \quad 1.048924$ $\begin{array}{lll}\text { C } 3.975360 & -3.006065 & -0.307087\end{array}$ C $3.258539-1.881669-0.727028$ C $3.214095-1.494710-2.191215$ $\begin{array}{llll}\text { C } 4.641800 & -1.311090 & -2.797766\end{array}$ $\begin{array}{llll}\text { C } 2.420644 & -2.525382 & -3.007819\end{array}$ H $2.690983-0.529477-2.271671$ H $5.239211-0.593248-2.212679$ H $5.185010-2.270080-2.818041$ H $4.563573-0.944845-3.8$ $\begin{array}{llll}\mathrm{H} & 4.563573 & -0.944845 & -3.835045\end{array}$ $\begin{array}{llll}H & 1.406573 & -2.656011 & -2.604372\end{array}$ $\begin{array}{llll}\text { H } 2.342832 & -2.204884 & -4.058530\end{array}$ H $2.919877-3.507052-2.986291$ H $4.540544-3.593210-1.035275$ H $4.550760-4.2753591 .356481$ H $3.212058-2.989982 \quad 3.031615$ C $0.249976 \quad 3.128743 \quad 2.763385$ H $1.252722 \quad 3.068636 \quad 2.324772$ H 0.2832603 .9414793 .505278 H $-0.820532 \quad 1.970107 \quad 4.261170$ H $-0.492589 \quad 1.073847 \quad 2.782225$ $\begin{array}{llll}\text { H } 2.401419 & 1.183217 & 2.344258\end{array}$ H $3.101551 \quad 0.281931 \quad 3.700576$ C $1.621846-0.839916 \quad 2.604703$ H $1.427344-1.514818 \quad 3.450582$ H $0.635950-0.6689212 .131233$ 79

$25 \mathrm{R}=\mathrm{Me} \mathrm{ET} / \mathrm{pi}$

Ni $0.028227 \quad 0.022744 \quad 0.031614$ N $1.980696 \quad 0.032352-0.026248$ $\begin{array}{llll}\text { N } 0.345993 & 2.018940 & 0.046692\end{array}$ $\begin{array}{llll}\text { C } 2.532754 & 1.209646 & -0.142491\end{array}$ $\begin{array}{llll}\text { C } 1.586378 & 2.336995 & -0.210002\end{array}$ C $2.807589-1.077155 \quad 0.373429$ $\begin{array}{llll}\text { C }-0.592575 & 3.076952 & 0.269028\end{array}$ C $4.0007261 .477078-0.144010$ $\begin{array}{lllll}\text { C } 2.088584 & 3.715240 & -0.488112\end{array}$ $\begin{array}{llll}\text { C } 0.005960 & -1.759450 & -0.683756\end{array}$ H $-1.004206-2.166180-0.824824$ н $0.621998-2.427335-0.063338$ H $0.488213-1.574717-1.659142$ $\begin{array}{llll}\text { C }-1.508712 & -0.661279 & 1.272177\end{array}$ $\begin{array}{llll}\text { C }-2.004920 & 0.114287 & 0.235028\end{array}$ H $-1.309859-0.244888 \quad 2.266939$ $\begin{array}{llll}\mathrm{H}-1.309859 & -0.244888 & 2.266939\end{array}$ H $-2.269902 \quad 1.1596850 .418974$ $\begin{array}{llll}\mathrm{H} & -2.449746 & -0.359129 & -0.646369\end{array}$ H $4.567059 \quad 0.603954 \quad 0.201054$
H $4.244478 \quad 2.337415 \quad 0.495572$ H $4.3285691 .729708-1.167727$ H $1.260668 \quad 4.418425 \quad-0.642546$ H $2.695195 \quad 4.0915020 .352730$ H $2.740677 \quad 3.709438-1.375935$ C $-0.508494 \quad 3.766240 \quad 1.499986$ C $-1.459199 \quad 4.762126 \quad 1.741733$ $\begin{array}{llll}\text { C }-2.439418 & 5.070086 & 0.803909\end{array}$ C $-2.494660 \quad 4.377324 \quad-0.402177$ C $-1.5765793 .363884-0.693282$ $\begin{array}{llll}C & -1.576579 & 3.363884 & -0.693282\end{array}$ C $-1.6005092 .630541-2.023099$ $\begin{array}{llll}C & -0.691373 & 3.317330 & -3.055217\end{array}$ $\begin{array}{llll}C & -3.011076 & 2.456119 & -2.592368\end{array}$ H $-1.185658 \quad 1.622895-1.843391$ H $0.352536 \quad 3.360708-2.713214$ $\begin{array}{llll}\mathrm{H} & -1.031257 & 4.349184 & -3.239745\end{array}$ H $-0.7148172 .769295-4.010362$ H $-2.9813941 .790071-3.467918$ H $-3.428884 \quad 3.416448-2.931796$ н $-3.7006752 .026253-1.851224$ H $-3.2676994 .628159-1.127837$ H $-3.168107 \quad 5.853414 \quad 1.014509$ H $-1.423790 \quad 5.299229 \quad 2.690976$ C $2.804431-1.352742 \quad 1.757240$ $\begin{array}{lll}\text { C } 3.641112 & -2.368424 & 2.215662\end{array}$ C $4.438050-3.1102781 .321860$ C $4.370768-2.845887-0.044522$ $\begin{array}{ll}\text { c } 4.370768-2.845887 & -0.044522\end{array}$ $\begin{array}{llll}C & 3.550839 & -1.827064 & -0.549988\end{array}$ C $3.480557-1.590834-2.049224$ $\begin{array}{llll}C & 4.842306 & -1.193970 & -2.640058 \\ C & 2.935080 & -2.827695 & -2.781981\end{array}$ C $2.935080-2.827695-2.781981$ H $2.779223-0.759698-2.226781$ H $5.269497-0.314069-2.138314$ H $5.565944-2.017952-2.539590$ H $4.740179-0.967667-3.71293$ H $1.966540-3.146345-2.37159$ H $2.809607-2.611387-3.854511$ H $3.632657-3.674885-2.689592$ H $4.965225-3.444559-0.736655$ H $5.088953-3.904101 \quad 1.696593$ H $3.658347 \quad-2.593182 \quad 3.283608$ C $0.494848 \quad 3.394699 \quad 2.560100$ 1.4987723 .2 $\begin{array}{llll}\mathrm{H} & 0.494848 & 3.394699 & 2.560100\end{array}$ $\begin{array}{llll}\mathrm{H} & 0.572660 & 4.217386 & 3.288077\end{array}$ C 0.1021562 .0905183 .291502 $\begin{array}{lllll}\mathrm{H} & -0.753078 & 2.308422 & 3.952521\end{array}$

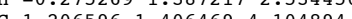
C 1.2065961 .4064694 .104894 H $1.6996612 .168803 \quad 4.737346$ B $0.4691390 .357528 \quad 5.171696$ F $1.439184-0.4912025 .771314$ F $-0.222514 \quad 1.1071206 .151269$ F $-0.481355-0.455701 \quad 4.446610$ C $2.293904 \quad 0.738236 \quad 3.242557$ H $2.592767 \quad 1.394462 \quad 2.394380$ H $3.204096 \quad 0.599071 \quad 3.850447$ C $1.871879-0.645256 \quad 2.704324$ H $1.693556-1.293009 \quad 3.574017$ $\begin{array}{llll}\text { H } & 0.893181 & -0.551421 & 2.204752\end{array}$ 80

$\mathrm{R}=\mathrm{Me} \mathrm{AC} / \mathrm{pi}$

Ti $0.013364 \quad 0.0192200 .035880$ N $1.9719670 .028152-0.003119$ $\begin{array}{llll}\mathrm{N} & 0.328864 & 2.023227 & 0.022355\end{array}$ C $2.5190771 .205275-0.136321$ C $1.5732922 .335472-0.215546$ $\begin{array}{llll}\text { C } 2.809297 & -1.074810 & 0.394868\end{array}$ $\begin{array}{llll}\text { C }-0.606743 & 3.084589 & 0.249544\end{array}$ $\begin{array}{llll}\text { C } 3.986954 & 1.471604 & -0.150384\end{array}$ $\begin{array}{llll}\text { C } 2.083327 & 3.711172 & -0.488825\end{array}$ $\begin{array}{llll}\text { C } 0.037566 & -1.788346 & -0.635114\end{array}$ H $-0.960000-2.211308-0.805315$ H $0.654438-2.428265 \quad 0.011676$ н $0.537712-1.587240-1.597666$ $\begin{array}{lll}C-1.454215 & -0.693372 & 1.287533\end{array}$ C $-2.0113290 .130318 \quad 0.298918$ C $-1.215436-0.290662 \quad 2.279165$ $\mathrm{H}-1.215436-0.290662 \quad 2.279165$ (. $\begin{array}{llll}H & -2.240927 & 1.176238 & 0.538507\end{array}$ $\mathrm{N}-3.326448-0.799006-1.737260$ $\begin{array}{lllll}\mathrm{N} & -3.326448 & -0.799006 & -1.737260 \\ \mathrm{H} & 4.555104 & 0.604446 & 0.206153\end{array}$ $\begin{array}{llll}\text { H } & 4.555104 & 0.604446 & 0.206153 \\ \text { H } & 4.234688 & 2.343338 & 0.471660\end{array}$ $\begin{array}{lllll}\text { H } & 4.234688 & 2.343338 & 0.471660 \\ H & 4.307603 & 1.705348 & -1.180912\end{array}$ $\begin{array}{lllll}\text { H } & 4.307603 & 1.705348 & -1.180912 \\ \text { H } & 1.259724 & 4.417762 & -0.649698\end{array}$ $\begin{array}{lllll}H & 1.259724 & 4.417762 & -0.649698\end{array}$ $\begin{array}{llll}\text { H } 2.687293 & 4.084557 & 0.355049\end{array}$ H $2.7408793 .700608-1.372673$ C $-0.517157 \quad 3.770783 \quad 1.483253$ $\begin{array}{llll}C & -1.463392 & 4.769985 & 1.729187\end{array}$ C $-2.444503 \quad 5.083538 \quad 0.794582$ $\begin{array}{lllll}C & -2.504940 & 4.394417 & -0.413116\end{array}$ $\begin{array}{ll}C-1.591417 & 3.379102-0.711807\end{array}$ C $-1.622002 \quad 2.661442-2.0$ $\begin{array}{llll}C & -1.622002 & 2.661442 & -2.049933\end{array}$ $\begin{array}{llll}C & -0.670536 & 3.325657 & -3.059760\end{array}$ C $-3.0263472 .550030-2.649207$ H $0.3705113 .326215-2.706471$

H $-0.971030 \quad 4.370943-3.237023$ H $-0.7046092 .788378-4.02033$ H $-3.010710 \quad 1.859173-3.504489$ H $-3.380973 \quad 3.525488-3.016950$ H $-3.756470 \quad 2.164521-1.923834$ H $-3.278359 \quad 4.648698-1.136811$ H $-3.169625 \quad 5.869203 \quad 1.008548$ H $-1.422962 \quad 5.305707 \quad 2.678931$ C $2.827247-1.331837-1.779576$ C $3.672741-2.3430562 .240983$ $\begin{array}{llll}C & 3.672741 & -2.343056 & 2.240983\end{array}$ $\begin{array}{llll}C & 4.446558 & -3.090772 & 1.356711\end{array}$ $\begin{array}{llll}\text { C } 4.364735 & -2.846279 & -0.018298\end{array}$ $\begin{array}{lll}c & 3.543192-1.833347 & -0.530442\end{array}$ C $3.466242-1.607850-2.027424$ C $4.852537-1.218390-2.633620$ $\begin{array}{llll}\text { C } 2.931050 & -2.846759 & -2.766340\end{array}$ $\begin{array}{llll}\text { H } 2.773871 & -0.772483 & -2.215865\end{array}$ H $5.288931-0.340574-2.131716$ H $5.564535-2.054065-2.531792$ H $4.742377-0.993448-3.707580$ H $1.958084-3.171083-2.365606$ H $2.812362-2.628092-3.840764$ H $3.631704-3.693114-2.669228$ H $4.953442-3.457865-0.706185$ H $5.100222-3.882288 \quad 1.731202$ H $3.698969-2.5502083 .312839$ C $0.484573 \quad 3.398867 \quad 2.545529$ C 0.4845733 .3988672 .545529 $\begin{array}{llll}\text { H } & 1.488831 & 3.273368 & 2.11414\end{array}$ 0.5645774 .2250623 .269124 C 0.0887932 .0997583 .284881 $\begin{array}{llll}\mathrm{H} & -0.770663 & 2.320250 & 3.939715\end{array}$ $\begin{array}{llll}\mathrm{H} & -0.284481 & 1.393038 & 2.528687\end{array}$ C $1.192014 \quad 1.420068 \quad 4.104129$ H $1.6650392 .180053 \quad 4.753670$ B $0.4667390 .333854 \quad 5.144415$ F $1.483099-0.559522 \quad 5.670275$ F $-0.181494 \quad 1.027192 \quad 6.185295$ F $-0.500516-0.447181 \quad 4.412703$ C $2.296608 \quad 0.774843 \quad 3.246351$ H $2.578752 \quad 1.432468 \quad 2.393547$ H $3.209687 \quad 0.659759 \quad 3.854246$ C $1.898144-0.620372 \quad 2.724770$ H $1.739165-1.257420 \quad 3.605410$ $\begin{array}{llll}\mathrm{H} & 1.739165 & -1.257420 & 3.605410 \\ \mathrm{H} & 0.915332 & -0.549042 & 2.231940\end{array}$ 80

$25 \mathrm{R}=\mathrm{Me} \mathrm{AC} / \mathrm{M}$

$203 \quad 0.113907-0.227272$ $\begin{array}{llll}\mathrm{N} 2.019294 & 0.081604 & -0.153534\end{array}$ $\begin{array}{llll}\text { N } & 0.424192 & 2.049112 & -0.015813\end{array}$ $\begin{array}{llll}\text { C } 2.602484 & 1.257864 & -0.061323\end{array}$ $\begin{array}{llll}\text { C } 1.682455 & 2.398098 & -0.023545\end{array}$ $\begin{array}{llll}\text { C } 2.821431 & -1.081259 & 0.127633\end{array}$ C $-0.610800 \quad 2.9785350 .296180$ C $4.075756 \quad 1.478201 \quad 0.021599$ $\begin{array}{llll}\text { C } 2.191209 & 3.798798 & 0.041521\end{array}$ C $-0.058379-1.775718-0.626866$ $\begin{array}{lllll}\text { н } & -0.843647 & -2.211137 & 0.006867\end{array}$ H $0.898371-2.288559-0.469453$ H $-0.364608-1.801180-1.686057$

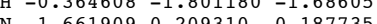
$\begin{array}{llll}N & -1.661909 & 0.209310 & -0.187735\end{array}$ $\begin{array}{llll}C & -2.815016 & 0.338073 & -0.085004\end{array}$ $\begin{array}{llll}C & -4.198724 & 0.573189 & 0.050318\end{array}$ $\begin{array}{llll}C & -5.137434 & -0.254667 & -0.433352\end{array}$ $\mathrm{H}-4.875410-1.171405-0.960915$ H $-6.193080-0.022606-0.302558$ H $4.607405 \quad 0.557638 \quad 0.290083$ H $4.316819 \quad 2.256150 \quad 0.759431$ H $4.450843 \quad 1.827480-0.957196$ H $1.364811 \quad 4.520456 \quad 0.014791$ H $2.7655893 .971676 \quad 0.966223$ H $2.874930 \quad 3.993452 \quad-0.801002$ C $-0.736576 \quad 3.392843 \quad 1.640844$ C $-1.837022 \quad 4.196149 \quad 1.962316$ $\begin{array}{lllll}C & -2.760459 & 4.580411 & 0.996347\end{array}$ C -2.603817 . $\begin{array}{llll}C & -2.603817 & 4.165852 & -0.325410\end{array}$ $\begin{array}{llll}C & -1.532764 & 3.351217 & -0.700556\end{array}$ C $-1.3152162 .928870-2.142841$ C $-0.3408313 .885465-2.850765$ C $-2.6089302 .805610-2.950575$ H $-0.840602 \quad 1.932522-2.119372$ H $0.632826 \quad 3.922042 \quad-2.342218$ H $-0.753053 \quad 4.907369-2.868329$ H $-0.1717563 .561319-3.889696$ H $-2.390550 \quad 2.370689-3.937545$ $\mathrm{H}-3.072174 \quad 3.789142-3.125009$ H $-3.3462892 .161815 \quad-2.448159$ H $-3.326423 \quad 4.485493-1.075903$ 
$\begin{array}{llll}\text { C } 2.886456 & -2.371878-3.196068\end{array}$ H $2.869104-0.390031-2.381810$ H $5.386040-0.116924-2.24659$ H $5.575519-1.765212-2.889541$ H $4.816813-0.508886-3.896636$ H $1.887096-2.646652-2.828591$ H $2.794303-2.024069-4.237330$ H $3.508699-3.281330-3.19478$ H $4.988309-3.309274-1.230281$ H $5.036082-4.0779641 .132313$ $\begin{array}{llll}H & 5.036082 & -4.077964 & 1.132313\end{array}$ $\begin{array}{llll}\text { H } 3.575402 & -2.976220 & 2.832764\end{array}$ C 0.2180162 .9558022 .718893 H 1.2505632 .9658022 .343466 H 0.1880633 .6917213 .538036 C $-0.072503 \quad 1.549848 \quad 3.306880$ H $-0.926799 \quad 1.628036 \quad 3.998576$ $\begin{array}{lllll}\text { H } & -0.399615 & 0.874633 & 2.496225\end{array}$ C $1.1333350 .953001 \quad 4.045459$ H $1.597703 \quad 1.754307 \quad 4.651026$ B $0.630901-0.153301 \quad 5.190891$ F $1.792656-0.864496 \quad 5.676098$ F $0.005093 \quad 0.622657 \quad 6.270398$ F $-0.304869-1.066666 \quad 4.650654$ C $2.213031 \quad 0.395150 \quad 3.112763$ H $2.466255 \quad 1.136222 \quad 2.318563$ H $3.1404460 .236453 \quad 3.688191$ $\begin{array}{llll}\text { H } 3.140446 & 0.236453 & 3.688191\end{array}$ $\begin{array}{llll}\text { C } 1.817719 & -0.945872 & 2.465231\end{array}$ $\begin{array}{llll}\mathrm{H} & 1.666608 & -1.669373 & 3.279196 \\ \mathrm{H} & 0.829346 & -0.841147 & 1.979492\end{array}$ 76

$26 \mathrm{R}=\mathrm{Me}$

$\begin{array}{llll}\text { Ni } 0.308645 & -0.045247 & 0.259851\end{array}$ N $1.9066830 .419894-0.519566$ N $0.029248 \quad 1.9466630 .198639$

$\begin{array}{llll}\text { C } 2.134883 & 1.708025 & -0.722562\end{array}$ C $0.993595 \quad 2.565677-0.436767$ $\begin{array}{llll}\text { C } 3.046393 & -0.461015 & -0.441767\end{array}$ $\begin{array}{lllll}\text { C } & -1.085647 & 2.693825 & 0.687998\end{array}$ $\begin{array}{llll}\text { C } 3.465718 & 2.315984 & -1.022217\end{array}$ C $1.034798 \quad 4.025387-0.749888$ $\begin{array}{llll}\text { C } 0.375604 & -1.868548 & -0.329459\end{array}$ H $-0.598071-2.288005-0.041132$ H $1.191495 \quad-2.416250 \quad 0.161559$ H $0.513507-1.826855-1.420506$ H $0.513507-1.826855-1.420506$ B $-0.410940-1.367694 \quad 3.087773$ B $-0.410940-1.3676943 .087773$ F $0.123598-2.536628 \quad 2.550521$ F $-1.678177-1.556213 \quad 3.610510$ $\begin{array}{llll}F & -0.795851 & -0.478607 & 1.727931\end{array}$ н $4.245684 \quad 1.558873-1.159082$ H $3.7723962 .977989-0.193722$ H $3.4089752 .942786-1.926315$ H $0.0705114 .505869-0.542423$ H $1.803471 \quad 4.528537-0.138306$ H $1.306119 \quad 4.190705 \quad-1.804326$ C $-0.945561 \quad 3.350902 \quad 1.930132$ $\begin{array}{lllll}C & -2.027675 & 4.102978 & 2.389493\end{array}$ C $-3.212358 \quad 4.181527 \quad 1.661212$ C -3.3409343 .4813260 .466355$ $\begin{array}{llll}C & -3.340934 & 3.481326 & 0.466355\end{array}$ $\begin{array}{llll}C & -2.284435 & 2.718423 & -0.040968\end{array}$ $\begin{array}{llll}C & -2.417744 & 1.953079 & -1.345898\end{array}$ C $-2.1517212 .858148-2.558213$ \begin{tabular}{c}
$c-3.7721741 .249567-1.479077$ \\
\hline
\end{tabular} $\mathrm{H}-1.642216-1.167843-1.338806$ H $-1.149154 \quad 3.308960-2.516632$ $\begin{array}{lllll}\text { H } & -2.888170 & 3.677342 & -2.596972\end{array}$ H $-2.229520 \quad 2.280986-3.493070$ н $-3.7669480 .594273-2.363383$ H $-4.5922861 .971840-1.612083$ H $-3.988174 \quad 0.637126-0.591740$ H $-4.283647 \quad 3.521233-0.079264$ H $-4.047400 \quad 4.771698 \quad 2.039377$ H $-1.941393 \quad 4.617779 \quad 3.348071$ $\begin{array}{llll}\text { c } 3.569551 & -0.769600 & 0.836704\end{array}$ C $4.764883-1.498042 \quad 0.870240$ C $5.387506-1.945361-0.289141$ $\begin{array}{llll}\text { C } & 5.387506 & -1.945361 & -0.289141 \\ \text { C } & .783736 & -1.711821 & -1.534951\end{array}$ $\begin{array}{llll}\text { C } & 4.783736 & -1.711821 & -1.534951 \\ \text { C } 3.600150 & -0.973795 & -1.627488\end{array}$ $\begin{array}{llll}\text { C } 3.600150 & -0.973795 & -1.627488 \\ \text { C } 2.928064 & -0.784073 & -2.977570\end{array}$ C $3.7529530 .083596-3.937565$ C $3.7529530 .083596-3.937565$ $\begin{array}{llll}\text { C } & 2.624884 & -2.139846 & -3.636548 \\ \text { H } & 1.966532 & -0.277780 & -2.803152\end{array}$ $\begin{array}{lllll}\mathrm{H} & 1.966532 & -0.277780 & -2.803152 \\ \mathrm{H} & 3.945026 & 1.086070 & -3.529433\end{array}$ $\begin{array}{llll}\text { H } & 3.945026 & 1.086070 & -3.529433 \\ \text { H } & 4.727185 & -0.385602 & -4.150325\end{array}$ $\begin{array}{llll}\text { H } & 4.727185 & -0.385602 & -4.150325 \\ \text { H } & 3.218980 & 0.200189 & -4.894001\end{array}$ H $2.069335-2.801387-2.953934$ H $2.023584-1.990463-4.548485$ H $3.554308-2.656826-3.928556$ H $5.243825-2.114233-2.440693$ H $6.322028-2.508017-0.232083$ H $5.197921-1.737718 \quad 1.843183$ $\begin{array}{llll}\text { C } 0.263498 & 3.129580 & 2.809635\end{array}$ H $1.1803173 .098768 \quad 2.204614$ $\begin{array}{llll}\text { H } & 1.180317 & 3.098768 & 2.204614 \\ \text { H } 0.374346 & 3.983406 & 3.495519\end{array}$ C $0.076957 \quad 1.798606 \quad 3.596156$ $\begin{array}{llll}\text { C } & 0.0 .299879 & 2.033997 \quad 4.605659\end{array}$ H $-0.739221 \quad 1.253659 \quad 3.102124$

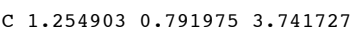
H 1.8641 C $2.202103 \quad 0.7880802 .509384$ н $1.408526-1.286997 \quad 4.363760$ H $0.041729-0.412042 \quad 5.017927$ H $1.603553 \quad 1.115396 \quad 1.658015$ H $2.991312 \quad 1.550874 \quad 2.642484$ $\begin{array}{llll}\text { C } 2.850504 & -0.561553 & 2.165101\end{array}$ H $3.562343-0.8345912 .960878$ $\begin{array}{llll}\text { H } 2.069347 & -1.341765 & 2.192179\end{array}$ 82

$6 \mathrm{R}=\mathrm{Me} \mathrm{ET/pi}$

Ni $-0.059719-0.0190910 .208567$ $\begin{array}{llll}1.684876 & 0.300970 & -0.610329\end{array}$ 0.1645312 .0418380 .549451 $\begin{array}{llll}\text { C } 2.058204 & 1.554435 & -0.743400\end{array}$ $\begin{array}{llll}\text { C } 1.192935 & 2.535843 & -0.087485\end{array}$ $\begin{array}{llll}\text { C } 2.636900 & -0.710687 & -0.99212\end{array}$ $\begin{array}{llll}\text { C }-0.535389 & 2.867929 & 1.496407\end{array}$ $\begin{array}{llll}\text { C } 3.355860 & 2.011784 & -1.332419\end{array}$ $\begin{array}{llll}\text { C } & 1.581009 & 3.980666 & -0.095529\end{array}$ $\begin{array}{llll}\text { C } 0.017104 & -1.909871 & -0.245243\end{array}$ H $-0.919669-2.408670 \quad 0.030911$ H $0.837588-2.267150 \quad 0.388092$ н $0.231149-2.006128-1.315905$ C $1.886335-1.0941434 .070898$ $\begin{array}{llll}\text { C } 1.886335 & -1.094143 & 4.070898\end{array}$ B $0.690148-2.8114$ F $0.690148 \quad-2.811416 \quad 2.624962$ F $-0.597330-1.659395 \quad 4.164697$ $\begin{array}{llll}\text { F } 0.110937 & -0.554694 & 2.243881\end{array}$ $\begin{array}{llll}C & -2.064911 & -0.069276 & 0.412999\end{array}$ $\begin{array}{llll}C & -1.734405 & 0.021709 & -0.940937\end{array}$ $\begin{array}{lllll}\mathrm{H} & -2.311732 & 0.814944 & 1.005984\end{array}$ H $-2.353705-1.022809 \quad 0.859324$ H $-1.796247-0.856865-1.585773$ H $-1.7428960 .979251-1.464984$ H $3.901344 \quad 1.191646-1.812069$ H $4.0031342 .428516-0.541287$ H $3.1898092 .816279 \quad-2.065594$ H $0.881052 \quad 4.583978 \quad 0.494787$ $\begin{array}{llllll}\text { H } 2.593367 & 4.124248 & 0.314267\end{array}$ H $1.5990914 .357953-1.131362$ C 0.061928 C $-0.6934203 .752124 \quad 3.734148$ $\begin{array}{llll}C & -0.693420 & 3.752124 & 3.734148\end{array}$ C -1.9760224 .2169153 .470883$ C -2.505105 4.073233 2.194281 C -1.7965043 .4165141 .184271$ $\begin{array}{llll}C & -2.334011 & 3.445208 & -0.236520\end{array}$ $\begin{array}{llll}C & -2.138885 & 4.847568 & -0.842651\end{array}$ $\begin{array}{llll}C & -3.805462 & 3.029777 & -0.351258\end{array}$ H $-1.729828 \quad 2.748884-0.833100$ H $-1.0849925 .160367-0.81475$ H $-2.7238235 .593446-0.28125$ н $-2.478240 \quad 4.863457-1.890432$ H $-4.1045802 .999982-1.410411$ H $-4.463435 \quad 3.754608 \quad 0.152156$ H $-3.990361 \quad 2.039414 \quad 0.086265$ H $-3.485483 \quad 4.493633 \quad 1.969488$ $-2.550225 \quad 4.717633 \quad 4.250697$ $\mathrm{H}-2.550225 \quad 4.717633 \quad 4.250697$ H $-0.251550 \quad 3.904818 \quad 4.720326$ C $3.495292-1.2362240 .001600$ $\begin{array}{llll}\text { C } 4.490909 & -2.122933 & -0.43040\end{array}$ C $3.609503-2.515254-1.757638$ C $3.688230-2.046045-2.699573$ $\begin{array}{llll}\text { C } 2.686210 & -1.143369 & -2.331245\end{array}$ $\begin{array}{llll}\text { C } 1.674753 & -0.674048 & -3.366075\end{array}$ $\begin{array}{llll}\text { C } 2.277026 & 0.312278 & -4.374797\end{array}$ C $1.047611-1.854242-4.124788$ H $0.865843-0.156601-2.827742$ H $2.669244 \quad 1.216213-3.888611$ $\begin{array}{llll}\text { H } 3.105046 & -0.157904 & -4.929671\end{array}$ H $1.512295 \quad 0.621862 \quad-5.104860$ $\begin{array}{lllll}\text { H } & 0.661118 & -2.622082 & -3.436317\end{array}$ н $0.215589-1.497447-4.754226$ H $1.781570-2.338404-4.790143$ H.752287-2.389913-3.734383 H $3.752287-2.389913-3.734383$ $\begin{array}{llll}\text { H } 5.399056 & -3.207351 & -2.059490\end{array}$ $\begin{array}{llll}4 & 5.175743-2.530996 & 0.315350\end{array}$ c 1.4641562 .6540113 .129601 H 2.0633262 .5203152 .223682 1.934307 3.4875153 .677605 C $1.494503 \quad 1.3573493 .984133$ H $1.651187 \quad 1.622450 \quad 5.04277$ H $0.492446 \quad 0.912036 \quad 3.928333$ C $2.5109790 .235868 \quad 3.604060$ H $3.4490590 .406190 \quad 4.166705$ C $2.8580710 .257146 \quad 2.096623$ H $2.640315-1.900606 \quad 4.022082$ H $1.653772-0.984079 \quad 5.146543$ H $1.935834 \quad 0.530715 \quad 1.587039$ H 3.6047991 .0471101 .889881 C $3.345696-1.069703 \quad 1.509282$ 3.345696 1.0697031 .509282 $\begin{array}{llll}\text { H } & 4.312160 & -1.344562 & 1.963629\end{array}$ $\mathrm{H} 23$

$\mathrm{AC} / \mathrm{pi}$

Ni $-0.056892-0.0467890 .205702$

N $1.698852 \quad 0.288771 \quad-0.605909$ N $0.141806 \quad 2.031703 \quad 0.54465$ $\begin{array}{llll}\text { C } 2.032606 & 1.545152 & -0.767454\end{array}$ $\begin{array}{llll}\text { C } 1.155207 & 2.526050 & -0.107594\end{array}$ $\begin{array}{llll}\text { C } 2.660505 & -0.712291 & -0.993210\end{array}$ C $-0.551266 \quad 2.853833 \quad 1.507734$ C $3.3105662 .022534-1.380040$ $\begin{array}{lllll}\text { C } 1.529149 & 3.972620 & -0.132994\end{array}$ $\begin{array}{llll}\text { C } 0.089062 & -1.963758 & -0.144944\end{array}$ H $-0.828212 \quad-2.461986 \quad 0.192441$ $\begin{array}{lllll}\text { H } & -0.828212 & -2.461986 & 0.19244 \\ \text { H } & 0.931386 & -2.251300 & 0.491743\end{array}$ $\begin{array}{llll}\text { H } & 0.931386 & -2.251300 & 0.491743\end{array}$ H $0.288638-2.119281-1.211759$ C $1.882729-1.105554 \quad 4.049979$ B $0.481702-1.5554073 .331687$ F $0.577246-2.7900002 .664647$ F $-0.629785-1.5261194 .174760$ $\begin{array}{llll}\text { F } & 0.109064 & -0.502468 & 2.217937\end{array}$ $\begin{array}{llll}\text { C } & -2.045497 & -0.037861 & 0.268521\end{array}$ C $-1.628699-0.164176-1.074751$ H $-2.342609 \quad 0.9189120 .699161$ H $-2.408936-0.927460 \quad 0.785407$ H $-1.694265-1.153395-1.536567$ C $-1.5387510 .862393 \quad-2.056412$ $\begin{array}{llll}\mathrm{N} & -1.414978 & 1.631360 & -2.929798\end{array}$ H $3.858000 \quad 1.212441-1.873722$ H $3.965244 \quad 2.442418-0.596552$ $\begin{array}{llll}\text { H } & 3.965244 & 2.442418 & -0.596552 \\ \text { H } & 0.856109 & 2.828716 & -2.103579\end{array}$ H $0.856109 \quad 4.5664210 .496325$ H $2.562251 \quad 4.127527 \quad 0.214310$ H $1.475892 \quad 4.345713-1.169430$ $\begin{array}{llll}\text { C } 0.052729 & 3.044497 & 2.776360\end{array}$ C $-0.700143 \quad 3.6778713 .769448$ C $-1.985642 \quad 4.1449903 .526938$ $\begin{array}{llll}\text { C }-2.512696 & 4.049473 & 2.245461\end{array}$ C $-1.805544 \quad 3.429657 \quad 1.211403$ C $-2.324965 \quad 3.546721-0.209370$ C $-2.1450894 .992059-0.713651$ $\begin{array}{llll}\text { C }-3.788661 & 3.124802 & -0.385186\end{array}$ H $-1.706329 \quad 2.911444-0.852638$ $\mathrm{H}-1.104355 \quad 5.333278-0.614885$ H $-2.7798695 .684991-0.138186$ $\mathrm{H}-2.431398 \quad 5.058792-1.774612$ $\mathrm{H}-4.0526253 .154948-1.453196$ $\begin{array}{llll}H & -4.052625 & 3.154948 & -1.453196\end{array}$ H -4.4688833 .8086600 .145755$ $\begin{array}{llll}H & -3.979634 & 2.107246 & -0.015882\end{array}$ H -3.4880064 .4866722 .030472$ $\begin{array}{llll}\mathrm{H}-2.558904 & 4.618181 & 4.324457 \\ \mathrm{H}-0.252510 & 3.805497 & 4.756541\end{array}$

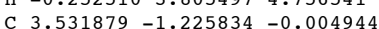
$\begin{array}{llll}\text { C } & 3.531879 & -1.225834 & -0.004944 \\ \text { C } 4.535682 & -2.099775 & -0.443954\end{array}$ $\begin{array}{llll}\text { C } 4.535682 & -2.099775 & -0.443954 \\ \text { C } 4.647595 & -2.493118 & -1.771441\end{array}$ $\begin{array}{llll}\text { C } & 4.647595 & -2.493118 & -1.771441 \\ \text { C } 3.710267 & -2.039703 & -2.705831\end{array}$ $\begin{array}{llll}\text { C } 2.699755 & -1.149110 & -2.33198\end{array}$ $\begin{array}{llll}\text { C } 1.665192 & -0.704926 & -3.354273\end{array}$ $\begin{array}{lllll}C & 2.221396 & 0.292601 & -4.377358\end{array}$ C $1.051196-1.900953-4.098710$ H $0.853844-0.197415 \quad-2.813494$ H $2.597399 \quad 1.208474-3.901673$ $\begin{array}{llll}\text { H } & 3.047994 & -0.156956 & -4.951345 \\ \text { H } & 1.427782 & 0.583125 & -5.082849\end{array}$ $\begin{array}{lllll}\mathrm{H} & 1.0427782 & 0.583125 & -5.082849\end{array}$ $\begin{array}{llll}\text { H } & 0.708170 & -2.684474 & -3.404099\end{array}$ H $0.191123-1.563522-4.70022$ H $1.778405-2.361025-4.788585$ H $3.767072-2.387292-3.739902$ H $5.443635-3.175321-2.078919$ $\begin{array}{llll}\text { H } & 5.232422 & -2.497640 & 0.296438\end{array}$ C $1.465826 \quad 2.630897 \quad 3.121847$ $\begin{array}{llll}\text { H } 2.050440 & 2.492711 & 2.207319\end{array}$ H $1.933708 \quad 3.480832 \quad 3.646319$ C $1.5314281 .352978 \quad 4.000777$ $\begin{array}{llll}\text { H } 1.734993 & 1.638962 & 5.045726\end{array}$ $\begin{array}{llll}\text { H } & 0.525880 & 0.912058 & 3.999928\end{array}$ C $2.528684 \quad 0.219539 \quad 3.600500$ $\begin{array}{lllll}\text { H } 3.469585 & 0.364789 & 4.165003\end{array}$ C $2.879510 \quad 0.254396 \quad 2.095057$ C 2.8795100 .2543962 .095057 H $2.614636-1.9295893 .969956$ $\begin{array}{llll}\text { H } & 1.675413 & -1.012827 & 5.132662\end{array}$ H 1.9561660 .5181031 .582723 C $3.390045-1.0617301 .504306$ H $4.365212-1.3163011 .951978$ $\begin{array}{llll}\text { H } & 4.365212 & -1.316301 & 1.951978 \\ \text { H } & 2.705017 & -1.867919 & 1.819566\end{array}$ 83

$26 \mathrm{R}=\mathrm{Me} \mathrm{AC} / \mathrm{N}$

Ni $0.083507 \quad 0.088823 \quad-0.327633$ $\mathrm{N} 1.900227 \quad 0.510394-0.623135$ $\begin{array}{llll}\mathrm{N} & -0.037817 & 2.036456 & -0.063330\end{array}$ C $2.202286 \quad 1.788509-0.592441$ $\begin{array}{lllll}\text { C } 1.062332 & 2.678560 & -0.349831\end{array}$ 
$\begin{array}{lll}C & 0.970136 & -0.513755 \quad 4.498152\end{array}$ B $-0.172776-1.587805 \quad 4.02899$ F $0.009705-2.830118 \quad 4.674075$ F $-1.506988-1.093466 \quad 4.352034$ F $-0.158793-1.7951492 .594472$ $\begin{array}{llll}\mathrm{N} & -1.571895 & -0.331284 & 0.28270\end{array}$ C $-2.402303-0.709662 \quad 1.005717$ C $-3.291564-1.160368 \quad 2.002672$ C $-4.594331-1.3941301 .796342$ H $-5.065739-1.2512600 .824391$ H $-5.065739-1.2512600 .82439$ H $-5.216692-1.742107 \quad 2.619525$ $\begin{array}{lllll}\text { H } & -2.784929 & -1.293737 & 2.97885\end{array}$ $\begin{array}{llll}H & 4.336123 & 1.578014 & -0.830803\end{array}$ $\begin{array}{llllll}H & \text { H } 3.828913 & 2.822403 & 0.337241\end{array}$ H $3.655191-3.138921-1.395913$ H $0.2694514 .665124 \quad-0.171186$ H $1.928519 \quad 4.476478 \quad 0.450669$ H $1.654671 \quad 4.503362-1.298858$ C $-1.175219 \quad 3.147652 \quad 1.793087$ $\begin{array}{llll}\text { C }-2.332803 & 3.761817 & 2.279850\end{array}$ C $-3.452845 \quad 3.942223 \quad 1.475591$ $\begin{array}{lllll}\text { C }-3.441671 & 3.492594 & 0.158694\end{array}$ C $-2.3148392 .866505-0.379126$ C $-2.286545 \quad 2.392807 \quad-1.820914$ C $-1.8139863 .512164-2.761823$ C $-3.626758 \quad 1.821810-2.290862$ $\begin{array}{llll}C & -3.626758 & 1.821810 & -2.290862\end{array}$ $\begin{array}{llll}\mathrm{H}-1.546802 & 1.575927 & -1.876826\end{array}$ $\begin{array}{llll}\mathrm{H}-0.815516 & 3.880357 & -2.483356\end{array}$ H $-2.510762-4.365285-2.724890$ $\mathrm{H}-1.7678873 .148055-3.800256$ $\mathrm{H}-3.509542 \quad 1.363485-3.284507$ H $-4.3945732 .605425-2.381556$ H $-3.994757 \quad 1.053592-1.595528$ $\begin{array}{lllll}\text { H } & -4.328695 & 3.625777 & -0.46000\end{array}$ H $-4.342667 \quad 4.425778 \quad 1.879376$ $\begin{array}{lllll}\mathrm{H} & -2.349974 & 4.091538 & 3.319987\end{array}$ $\begin{array}{llll}\text { C } 3.465920 & -0.854713 & 0.717185\end{array}$ $\begin{array}{llll}\text { C } 4.572361 & -1.712807 & 0.714282\end{array}$ $\begin{array}{llll}\text { C } 5.166435 & -2.168872 & -0.470458\end{array}$ C $4.621818-1.787346-1.696661$ C $3.520685-0.930804-1.755212$ C $2.926240-0.549980-3.102886$ $\begin{array}{llll}\text { C } 2.926240 & -0.5349980 & -3.102886\end{array}$ $\begin{array}{llll}\text { C } 3.802211 & 0.467239 & -3.850717\end{array}$ $\begin{array}{llll}C & 2.674795 & -1.780644 & -3.985789\end{array}$ $\begin{array}{lllll}\mathrm{H} & 1.949672 & -0.078767 & -2.91217\end{array}$ $\begin{array}{lllll}\text { H } & 3.942091 & 1.392127 & -3.273093 \\ \text { H } & 4.799306 & 0.044077 & -4.054550\end{array}$ $\begin{array}{lllll}\text { H } & 4.799306 & 0.044077 & -4.054550 \\ \text { H } & 3.340049 & 0.731699 & -4.814955\end{array}$ $\begin{array}{llll}\text { H } & 3.340049 & 0.731699 & -4.814955 \\ \text { H } & 2.077019 & -2.538672 & -3.459522\end{array}$ $\begin{array}{llll}\text { H } & 2.077019 & -2.538672 & -3.459522 \\ \text { H } & 2.133289 & -1.482344 & -4.896671\end{array}$ H $3.619296-2.248930-4.302349$ H $5.058006-2.166104-2.62217$ н $6.032322-2.833729-0.430506$ H $4.969914-2.044740 \quad 1.674722$ C $-0.019696 \quad 2.892503 \quad 2.732590$ н $0.930733 \quad 2.948565 \quad 2.186939$ н $0.011270 \quad 3.707728 \quad 3.472729$ C $-0.123693 \quad 1.520585 \quad 3.460776$ H $-0.597885 \quad 1.666042 \quad 4.444457$ $\begin{array}{lllll}\mathrm{H} & -0.597885 & 1.666042 & 4.444457 \\ \mathrm{H}-0.810960 & 0.868205 & 2.902187\end{array}$ $\begin{array}{lllll}\mathrm{H} & -0.810960 & 0.868205 & 2.902187\end{array}$ $\begin{array}{llll}\text { C } 1.200317 & 0.744495 & 3.639870\end{array}$ $\begin{array}{llll}\mathrm{H} & 1.924671 & 1.405341 & 4.162440\end{array}$ $\begin{array}{llll}\text { C } & 1.768228 & 0.462681 & 2.236117 \\ \text { H } & 1.932020 & -1.047009 & 4.610801\end{array}$ H $1.932020-1.0470094 .610801$ H $0.710694-0.180548 \quad 5.520661$ H $0.9182590 .097927 \quad 1.629388$ H $2.0974691 .419641 \quad 1.802996$ C $2.888258-0.556399 \quad 2.101749$ H $3.734771-0.2797462 .755274$ $\mathrm{H}$
79 $27 \mathrm{R}=\mathrm{Me}$

Ni $-0.004932 \quad 0.153045 \quad 0.195389$ $\begin{array}{llll}\mathrm{N} & 1.850495 & 0.064802 & 0.143664\end{array}$ $\begin{array}{lllll}\mathrm{N} & 0.367314 & 2.074004 & -0.139921\end{array}$ C $2.497901 \quad 1.189104-0.110499$ $\begin{array}{llll}\text { C } & 1.627291 & 2.318432 & -0.399778\end{array}$ $\begin{array}{lllr}\text { C } & 1.627291 & 2.318432 & -0.399778 \\ \text { C } & 2.588374 & -1.029533 & 0.713258\end{array}$ $\begin{array}{llll}\text { C } & 2.588374 & -1.029533 & 0.713258 \\ \text { C } 3.972519 & 3.146101 & -0.200877\end{array}$ $\begin{array}{llll}\text { C } 3.972344 & 1.390120 & 0.009268\end{array}$ $\begin{array}{llll}\text { C } & 3.972344 & 1.390120 & 0.009268 \\ \text { C } & 2.186544 & 3.622066 & -0.867719\end{array}$ $\begin{array}{llll}\text { C } & 2.186544 & 3.622066 & -0.867719 \\ \text { C }-0.288987 & -1.728159 & -0.113382\end{array}$ $\begin{array}{lllll}\text { C } & -0.288987 & -1.728159 & -0.113382 \\ \text { H } & -1.334618 & -1.945766 & 0.139330\end{array}$ $\begin{array}{llll}\mathrm{H} & -1.334618 & -1.945766 & 0.139330 \\ \mathrm{H} & 0.414456 & -2.359870 & 0.448270\end{array}$ H $-0.111684-1.825557-1.198430$ $\begin{array}{lllll}\text { H } & 4.467893 & 0.521678 & 0.458548\end{array}$ H $4.188950 \quad 2.276202 \quad 0.627532$ H $4.4188691 .576659-0.982036$ H $1.387599 \quad 4.338961-1.094088$ H $2.838397 \quad 4.068547-0.098372$ H $2.8062363 .473958-1.766744$ C $-0.668214 \quad 3.972127 \quad 0.939603$ $\begin{array}{lllll}\text { C } & -0.668214 & 3.972127 & 0.939603\end{array}$ $\begin{array}{llll}C & -1.563388 & 5.039101 & 0.897773\end{array}$ $\begin{array}{llll}C & -2.351981 & 5.268276 & -0.22924\end{array}$ $\begin{array}{lll}C & -2.272430 & 4.410319-1.319845\end{array}$ $\begin{array}{llll}\text { C } & -1.385915 & 3.327172 & -1.326869 \\ \text { C } & -1.349444 & 2.357275 & -2.492443\end{array}$
C $-1.057582 \quad 3.052173-3.827998$ C $-2.6540731 .547816-2.550719$ н $-0.530945 \quad 1.644442-2.303512$ H $-0.1156883 .620973-3.790139$ H $-1.864053 \quad 3.751113-4.098856$ H $-0.980553 \quad 2.305251-4.633387$ $\mathrm{H}-2.594972 \quad 0.786536-3.344407$ H $-3.511080 \quad 2.204691-2.770446$ $\mathrm{H}-2.8432251 .046717-1.590072$ $4.9176254 .577457-2.183033$ $\begin{array}{lll}\mathrm{H}-2.917625 & 4.577457-2.583033\end{array}$ $\begin{array}{lllll}\mathrm{H} & -3.049662 & 6.105982 & -0.24515\end{array}$ $\mathrm{H}-1.6565245 .6872741 .770907$ C $3.156912-2.2706022 .693902$ c $4.057465-3.0319201 .956979$ $\begin{array}{llll}\text { C } 4.174429 & -2.823159 & 0.570626\end{array}$ $\begin{array}{llll}\text { C } 3.438293 & -1.824060 & -0.074775\end{array}$ C $3.516273-1.679208-1.58333$ $\begin{array}{llll}\text { C } 4.973179-1.464681 & -2.093527\end{array}$ $\begin{array}{llll}\text { C } 2.896374 & -2.902256 & -2.277335\end{array}$ H $2.924859-0.798848-1.875116$ H $5.457949-0.608203-1.600307$ H $5.589829-2.358992-1.906069$ H $4.963274-1.284173-3.181010$ H $1.862829-3.068788-1.940029$ \begin{tabular}{llll} 
H 2.890746 & -2.758229 & -3.36972 \\
\hline & 3.476245 & -3.813342 & -2.055998
\end{tabular} $\begin{array}{llll}\text { H } 3.476245 & -3.813342 & -2.05599\end{array}$ $\begin{array}{llll}\text { H } & 4.844231 & -3.460712 & -0.01165\end{array}$ H $4.647146-3.8144612 .439902$ H $0.0645913 .598137 \quad 2.758429$ C 0.0645913 .5981372 .206961 $\begin{array}{llll}\text { H } & 1.120784 & 3.368182 & 1.994127\end{array}$ H $0.063136 \quad 4.449634 \quad 2.905965$ $\begin{array}{llll}\text { C }-0.654075 & 2.375182 & 2.811865\end{array}$ H $-1.592324 \quad 2.735293 \quad 3.267453$ H $-0.985977 \quad 1.744128 \quad 1.982111$ C $0.065765 \quad 1.491252 \quad 3.838219$ C $-0.986858 \quad 0.499790 \quad 4.410867$ H $-0.498344-0.108163 \quad 5.194295$ H $-1.720474 \quad 1.127631 \quad 4.946342$ H $0.349321 \quad 2.135708 \quad 4.691984$ $\begin{array}{lllll}\text { C } 1.403219 & 0.848641 & 3.341132\end{array}$ C $1.827352 \quad 1.4730942 .537590$ $\begin{array}{llll}\text { H } 1.827352 & 1.4730942 .537590\end{array}$

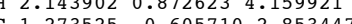
$\begin{array}{lllll}C & 1.273525 & -0.605710 & 2.853447\end{array}$ 19.6609142 .7180688 H $0.392919-0.660914 \quad 2.190688$ C $-1.792110-0.4635013 .490142$ H $-1.173824-1.309063 \quad 3.15320$ $\begin{array}{llll}\mathrm{H}-2.517324 & -0.953678 & 4.174394\end{array}$ B $-2.766551-0.048682 \quad 2.285782$ F $-3.467086-1.103460 \quad 1.76017$ $\begin{array}{lllll}\text { F }-1.773038 & 0.334413 & 0.706646\end{array}$ $\mathrm{F}$

$27 \mathrm{R}=\mathrm{Me} \mathrm{ET/pi}$

Ni $-0.132220 \quad-0.052863 \quad 0.280944$ $\begin{array}{lllll}\mathrm{N} & 1.687088 & 0.230278 & -0.365973\end{array}$

N $0.071780 \quad 2.046835 \quad 0.569301$

$\begin{array}{llll}\text { C } & 2.057617 & 1.477915 & -0.552407\end{array}$

$\begin{array}{llll}\text { C } & 1.140954 & 2.494169 & -0.038398 \\ \text { C } & 2.658955 & -0.792760 & -0.641254\end{array}$ $\begin{array}{llll}\text { C } & 2.658955 & -0.792760 & -0.641254 \\ \text { C } & -0.615605 & 2.974717 & 1.431544\end{array}$ C $3.372419 \quad 1.913880-1.119630$ C $1.5146403 .939350-0.170413$ C $-0.030271-1.963710-0.104258$ H $-0.998291-2.4350340 .101868$ н $0.729254-2.321840 \quad 0.600768$ н $0.283777-2.095227-1.145627$ $\begin{array}{llll}\text { C } 1.043310 & -1.723839 & 4.317644\end{array}$ B $-0.425930-1.377122 \quad 3.719573$ F $-1.155413-2.5446113 .458790$ F $-1.172239-0.450888 \quad 4.451257$ F $-0.283062-0.717133 \quad 2.280048$ C $-2.149592-0.068501-0.170861$ C $-2.149592-0.0685010 .170861$ $\begin{array}{llll}C & -1.606257 & -0.005794 & -1.113696\end{array}$ $\begin{array}{lllll}\mathrm{H} & -2.461491 & 0.827387 & 0.710607\end{array}$ $\mathrm{H}-2.523590-1.0085830 .579916$ $\mathrm{H}-1.586333-0.894121-1.747675$ $\mathrm{H}-1.500092-0.941506-1.648278$ H $4.032101 \quad 1.063929-1.325039$ H $3.8877752 .590320-0.419154$ H $3.2198492 .480696-2.052926$ H $0.729237 \quad 4.594430 \quad 0.224102$ H $2.448015 \quad 4.167819 \quad 0.368750$ H $1.691875 \quad 4.180672-1.231072$ $\begin{array}{lllll}\text { C } & 0.035251 & 3.330981 & 2.641503\end{array}$ C $-0.639789 \quad 4.170511 \quad 3.528036$ C $-1.902011 \quad 4.674907 \quad 3.234409$ $\begin{array}{llll}\text { C }-2.496037 & 4.363473 & 2.019242\end{array}$ C $-1.866563 \quad 3.523973 \quad 1.093449$ C $-2.4900373 .339166-0.277730$ C $-2.4900373 .339166-0.277730$ $\begin{array}{llll}C & -2.215498 & 4.565446 & -1.166382\end{array}$ $\begin{array}{lll}C & -3.996139 & 3.054143-0.227407\end{array}$ H $-1.996062 .482608-0.750439$ $\begin{array}{llll}\mathrm{H} & -1.137532 & 4.746646 & -1.288448 \\ \mathrm{H}-2.661599 & 5.470044 & -0.722988\end{array}$

H $-2.655668 \quad 4.419972-2.165755$ H $-4.358812 \quad 2.774086-1.228146$ H $-4.560675 \quad 3.945420 \quad 0.086575$ H $-4.237063 \quad 2.238027 \quad 0.468424$ H $-3.468855 \quad 4.789275 \quad 1.774223$ H $-2.414959 \quad 5.321076 \quad 3.946968$ H $-0.157220 \quad 4.426445 \quad 4.472624$ C $3.315816-1.3340870 .484869$ $\begin{array}{llll}C & 4.338807 & -2.255155 & 0.258723\end{array}$ C $4.678029-2.660850-1.037025$ $\begin{array}{lll}\text { C } 4.678029 & -2.660850 & -1.037025\end{array}$ $\begin{array}{lll}\text { C } 3.954102-2.167888 & -2.124234\end{array}$ $\begin{array}{llll}\text { C } 2.930492 & -1.228773 & -1.949316\end{array}$ C $3.133786-0.755162-3.155194$ $\begin{array}{llll}C & 3.008482 & -0.034444 & -4.192475\end{array}$ C $1.400539-1.928123-3.828399$ $\begin{array}{llll}\text { H } & 1.370290 & -0.046823 & -2.797973\end{array}$ H $3.5480520 .819341-3.759786$ H $3.756715-0.722137-4.616986$ $\begin{array}{llll}\text { н } & 2.385395 & 0.334358 & -5.022229\end{array}$ H $0.780285-2.486887-3.113462$ н $0.752983-1.557144-4.638113$ H $2.118618-2.637174-4.269624$ н $4.187140-2.523926-3.129420$ H $5.479310-3.386573-1.195335$ H $4.860481-2.682627 \quad 1.116792$ C $1.384638 \quad 2.784772 \quad 3.047104$ H $2.0718812 .753097 \quad 2.0189260$ H 2.0718812 .7530972 .189260 $\begin{array}{llll}H & 1.839743 & 3.474312 & 3.778007\end{array}$ C $1.2157151 .381144 \quad 3.635625$ H. $0.6519501 .469105 \quad 4.579859$ $\begin{array}{ll}0.550202 & 0.8254472 .971194\end{array}$ $\begin{array}{lllll}\text { C } 2.479246 & 0.549810 & 3.897466\end{array}$ $\begin{array}{llll}\text { C } 2.093821 & -0.672677 & 4.779553\end{array}$ H $3.028142-1.203527 \quad 5.044144$ H $1.726766-0.238090 \quad 5.726339$ H $3.150641 \quad 1.162852 \quad 4.530976$ C $3.311406 \quad 0.215423 \quad 2.625211$ H $3.286182 \quad 1.078348 \quad 1.936832$ H $4.372019 \quad 0.092279 \quad 2.909000$ C $2.845050-1.056471 \quad 1.891834$ H $3.137603-1.9247732 .499592$ H $1.748744-1.049051 \quad 1.885789$ H $1.748744-1.049051 \quad 1.885789$ $\begin{array}{llll}\mathrm{H} & 1.508094 & -2.460119 & 3.641204\end{array}$ 86

$27 \mathrm{R}=\mathrm{Me} \mathrm{AC} / \mathrm{pi}$

$148068-0.084664 \quad 0.299091$ N $1.690372 \quad 0.210600-0.342404$ $\begin{array}{llll}\mathrm{N} & 0.050960 & 2.038858 & 0.581780\end{array}$ $\begin{array}{llll}\text { C } 2.027810 & 1.456299 & -0.563855\end{array}$ $\begin{array}{llll}\text { C } 1.103205 & 2.478633 & -0.050284\end{array}$ $\begin{array}{llll}\text { C } 2.667085 & -0.805031 & -0.624161\end{array}$ $\begin{array}{llll}\text { C }-0.631626 & 2.972614 & 1.447370\end{array}$ C $3.3313531 .904752-1.144487$ $\begin{array}{lllll}\text { C } 1.467807 & 3.920280 & -0.215846\end{array}$ C $-0.025781-2.020398 \quad 0.014584$ н $-1.004860-2.467280 \quad 0.224756$ H $0.709409-2.3363510 .762446$ H $0.321827-2.207474-1.007975$ C $0.321827-2.207474-1.007975$ C $1.047376-1.7117234 .293224$ B $-0.425925-1.364346 \quad 3.713087$ F $-1.136447-2.5209330 .388119$ $F-1.180315-0.466570-4.456649$ $\begin{array}{lll}F-0.308686 & -0.623073 & 2.259633\end{array}$ $\begin{array}{llll}C & -2.115813 & -0.033795 & -0.007935\end{array}$ $\begin{array}{llll}\text { C }-1.448041 & -0.116001 & -1.251197\end{array}$ $\begin{array}{llll}\text { H }-2.472129 & 0.910575 & 0.400422\end{array}$ $\begin{array}{lllll}\mathrm{H} & -2.592627 & -0.932099 & 0.386136\end{array}$ H $-1.441492-1.082116-1.764249$ $\begin{array}{llll}\text { C } & -1.127784 & 0.954617 & -2.134072\end{array}$ N $-0.783060 \quad 1.763534 \quad-2.907270$ H $3.9741291 .061037-1.415357$ H $3.8738882 .528454 \quad-0.414899$ H $3.157958 \quad 2.530824 \quad-2.033787$ H $0.729969 \quad 4.578102 \quad 0.256819$ $\begin{array}{llll}H & 0.729969 & 4.578102 & 0.256819\end{array}$ $\begin{array}{lllll}H & 0.729969 & 4.578102 & 0.256819\end{array}$ $\begin{array}{llll}\mathrm{H} & 1.522719 & 4.151883 & -1.292507\end{array}$ C 0.0266113 .3208922 .655852

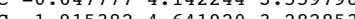
C $-1.915382 \quad 4.6419203 .282853$ $\begin{array}{llll}C & -2.507420 & 4.356560 & 2.060731\end{array}$ C -1.8780173 .5388691 .115475$ $\begin{array}{llll}\text { C }-2.487922 & 3.419102 & -0.268057\end{array}$ C $-2.203907 \quad 4.687301-1.092975$ C $-3.993871 \quad 3.130335-0.255622$ H $-1.9837462 .599725-0.790225$ H $-1.126132 \quad 4.888879-1.168642$ H $-2.6834615 .564850-0.629826$ н $-2.597414 \quad 4.569035-2.114151$ H $-4.3363132 .907250 \quad-1.277594$ $\mathrm{H}-4.565842 \quad 4.001853 \quad 0.097904$ 
C $4.699291-2.653373-1.035003$ C $3.954821-2.175266-2.115442$ C $2.922022-1.247146-1.934909$ $\begin{array}{lll}\text { C } 2.098767 & -0.791250 & -3.126826\end{array}$ C $2.936546-0.024657-4.179892$ C $1.402903-1.976720-3.816683$ H $1.318624-0.105254 \quad-2.768025$ H $3.442934 \quad 0.850702-3.748890$ H $3.705867-0.680465-4.619944$ H $2.2777470 .325272-4.990874$

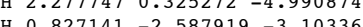
$\begin{array}{llll}H & 0.827141 & -2.587919 & -3.10336\end{array}$ - $0.715920-1.608587-4.596804$ H $2.139121-2.640794-4.300913$ H $4.178834-2.535996-3.122173$ H $5.507345-3.369651-1.202061$ $\begin{array}{llll}\text { H } & 4.916177 & -2.662251 & 1.112401\end{array}$ C $1.389295 \quad 2.793780 \quad 3.041461$ $\begin{array}{llll}\text { H } 2.060608 & 2.762328 & 2.170775\end{array}$ H $1.849722 \quad 3.496585 \quad 3.756158$ C $1.2419631 .395948 \quad 3.647510$ H $0.693953 \quad 1.492458 \quad 4.600453$ H $0.567072 \quad 0.834556 \quad 2.998977$ C $2.506443 \quad 0.566869 \quad 3.896898$ C $2.102421-0.675956 \quad 4.781839$ H $3.032048-1.217038 \quad 5.045468$ H $1.731255-0.2415615 .728046$ $\begin{array}{lllll}\text { H } & 1.731255 & -0.241561 & 5.728046\end{array}$ $\begin{array}{llll}H & 3.177500 & 1.166887 & 4.542170\end{array}$ $\begin{array}{llll}\text { C } 3.339809 & 0.234188 & 2.626922\end{array}$ . 3.3037081 .0923921 .933471 H 4.4022130 .1236022 .908045 C $2.883170-1.0466401 .903810$ $\begin{array}{lllll}\text { H } 3.185201 & -1.908697 & 2.515956\end{array}$ H $1.787270-1.0428571 .902615$ H $0.810235-2.319274 \quad 5.190355$ $\begin{array}{llll}\text { H } & 1.512265 & -2.439843 & 3.608935\end{array}$ 86

$27 \mathrm{R}=\mathrm{Me} \mathrm{AC} / \mathrm{N}$

Ni $0.387769 \quad 0.138608 \quad-0.490789$ N 2.241722 $0.448217 \quad-0.329804$ N $0.3403572 .096395-0.404697$ C $2.617357 \quad 1.708189-0.353375$ C $1.5122422 .666575-0.447104$ C $3.223831-0.5431220 .029690$ $\begin{array}{llll}\text { C } 3.223831 & -0.543122 & 0.029690\end{array}$ $\begin{array}{llll}\text { C } & -0.845820 & 2.859212 & -0.188874\end{array}$ $\begin{array}{llll}\text { C } 4.013832 & 2.200967 & -0.180378\end{array}$ C $0.536619-1.772887-0.770765$ C $0.536619-1.772887-0.770765$ H $0.027821-2.2423470 .085341$ $\begin{array}{lllll}\mathrm{H} & 1.591513 & -2.068674 & -0.825118\end{array}$ H $-0.000230-1.990798-1.706946$ C $-0.223596-1.221273 \quad 4.618940$ B $-1.503673-1.416801 \quad 3.63431$ F $-1.117156-1.349158 \quad 2.225830$ $\begin{array}{llll}F & -2.102784 & -2.699342 & 3.810945\end{array}$ F $-2.521864-0.423786 \quad 3.843992$ N $-1.411869-0.098971-0.416421$ $\begin{array}{llll}\text { C }-2.477566 & -0.253949 & 0.026202\end{array}$ $\begin{array}{llll}C & -3.784888 & -0.378198 & 0.538763\end{array}$ $\begin{array}{llll}\mathrm{C}-4.095669 & -1.274229 & 1.48922 \\ \mathrm{H}-4.509251 & 0.346455 & 0.159933\end{array}$ $\begin{array}{llll}\mathrm{H} & -4.509251 & 0.346455 & 0.159933\end{array}$ $\begin{array}{lllll}\mathrm{H} & -5.107407 & -1.298900 & 1.89195\end{array}$ H $-3.358395-1.9621001 .900613$ H 4.7062201 .3905300 .073920 H 4.0562012 .9643300 .612626 H $4.3588722 .686670-1.109243$ H $0.819253 \quad 4.693479-0.567623$ H $2.288805 \quad 4.478365 \quad 0.415244$ H $2.405304 \quad 4.388834 \quad-1.349283$ C $-1.102501 \quad 3.282636 \quad 1.132521$ $\begin{array}{llll}\text { C }-2.306514 & 3.949466 & 1.368611\end{array}$ C $-3.214422 \quad 4.187654 \quad 0.340167$ $\begin{array}{llll}C & -2.937488 & 3.747115 & -0.950559\end{array}$ C $-1.752789 \quad 3.063229-1.239465$ C $-1.4287892 .594187 \quad-2.646231$ C $-0.8306043 .736995-3.482011$ C $-0.8306043 .736995-3.482011$

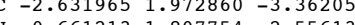
$\begin{array}{llll}\mathrm{H} & -0.661213 & 1.807754 & -2.556131\end{array}$ H $0.0735304 .151838-3.011870$ $\mathrm{H}-1.558728-4.556935-3.590851$ H $-0.56224903 .378718-4.488403$ H $-2.3166671 .559297-4.332177$ H $-3.415522 \quad 2.719038-3.563377$ $\mathrm{H}-3.070470 \quad 1.158258-2.767962$ H $-3.6579963 .933823-1.74730$ H $-4.146309 \quad 4.714239 \quad 0.548007$ $\begin{array}{llll}\mathrm{H}-2.534878 & 4.275652 & 2.384793\end{array}$ $\begin{array}{llll}\text { C } 3.176681 & -1.018318 & 1.358288\end{array}$ $\begin{array}{llll}\text { C } 4.177416 & -1.906130 & 1.753637\end{array}$ $\begin{array}{llll}\text { C } 5.169655 & -2.338246 & 0.855735\end{array}$ C $5.134270-1.906455-0.465560$ C $4.157557-1.006726-0.909500$ c $4.088067-0.625698-2.377721$ C $5.405236-0.002479-2.377721$ $\begin{array}{llll}C & 0.005236 & -0.002479 & -2.89919\end{array}$ $\begin{array}{llll}C & & \\ C & .705236 & -0.002479 & -2.899194\end{array}$ $\begin{array}{llll}\text { H } 3.290379 & 0.122446 & -2.499757\end{array}$ $\begin{array}{llll}\text { H } & 5.708441 & 0.872001 & -2.303259 \\ \text { H } & 6.227599 & -0.735190 & -2.866148\end{array}$
H $5.2803330 .314388-3.947202$ H $2.754517-2.285796-2.895992$ H $3.601210-1.555309-4.28829$ н $4.480038-2.625894-3.167076$ H $5.876214-2.280759-1.174077$ H $5.944146-3.033513 \quad 1.188896$ H $4.169029 \quad-2.284834 \quad 2.777219$ C $-0.159575 \quad 2.971594 \quad 2.269643$ H $0.8824883 .161743 \quad 1.964278$ $4-0.3635573 .6726403 .09$ $\begin{array}{lllll}\mathrm{H} & -0.363557 & 3.672640 & 3.095823\end{array}$ $\begin{array}{llll}\text { C }-0.288645 & 1.524109 & 2.783612\end{array}$ $\begin{array}{llll}\mathrm{H} & -1.269878 & 1.402628 & 3.270617\end{array}$ $\begin{array}{lllll}H & -0.320706 & 0.825718 & 1.934767\end{array}$ C $0.8357951 .124353 \quad 3.761159$ $\begin{array}{llll}C & 0.354336 & 0.185886 & 4.896868\end{array}$ H $1.197902 \quad 0.0976605 .610083$ H $-0.427467 \quad 0.756353 \quad 5.432463$ H $1.130428 \quad 2.053121 \quad 4.290645$ C $2.131216 \quad 0.680974 \quad 3.029500$ H $2.403467 \quad 1.4688562 .302699$ H 2.9609140 .6498463 .759366 C $2.053757-0.677177 \quad 2.308790$ H $2.016895-1.4735393 .064437$ H $1.092160-0.756488 \quad 1.774084$ H $-0.538847-1.609320 \quad 5.606453$ H $0.572972-1.915464 \quad 4.296974$ 73

\section{$28 \mathrm{R}=\mathrm{Me}$}

Ni $0.188308 \quad 0.2536850 .291888$ $\begin{array}{llll}N & 0.441184 & 1.993079 & -0.215411\end{array}$ C $1.700152 \quad 2.403202-0.325227$ $\begin{array}{llll}\text { C } 2.114421 & 3.837932 & -0.353934\end{array}$ $\begin{array}{llll}\text { C }-0.562071 & 2.893984 & 0.287696\end{array}$ $\begin{array}{llll}\text { C }-0.587839 & 3.081147 & 1.691860\end{array}$ C $0.478752 \quad 2.520486 \quad 2.606371$ H 0.7838633 .3501563 .271989 H $1.370368 \quad 2.303574 \quad 2.006838$ C $0.204215 \quad 1.285294 \quad 3.520463$ H $-0.409710 \quad 1.676541 \quad 4.358852$ B $-0.7667550 .035696 \quad 3.096419$ F $-0.735192-0.9552314 .094897$ F $-0.287919-0.8003391 .819650$ F -2.0565770 .4179342 .748639$ F $-2.0565770 .417934 \quad 2.748639$ C $1.5552020 .905295 \quad 4.195268$ 1.9313681 .7940734 .736092 $\begin{array}{ll}1.350610 & 0.139653 \\ 4.961434\end{array}$ C 2.6993840 .4070173 .287561 H 3.6520760 .4856403 .840809 H $2.810296 \quad 1.072848 \quad 2.419993$ C $2.516116-1.047852 \quad 2.821084$ H $2.820404-1.718027 \quad 3.640564$ H $1.446849-1.2429802 .667658$ C $3.213930-1.5169551 .566840$ $\begin{array}{llll}\text { C } 2.976568 & -0.968274 & 0.279640\end{array}$ N $2.161933 \quad 0.200124 \quad 0.188071$ C $3.496420-1.580155-0.885320$ $\begin{array}{llll}\text { C } 4.308615 & -2.707906 & -0.735233\end{array}$ $\begin{array}{llll}\text { C } 4.614835 & -3.213725 & 0.521084\end{array}$ C $4.062701-2.6228131 .652968$ $\begin{array}{llll}\text { C } 4.062701 & -2.622813 & 1.652968\end{array}$ $\begin{array}{llll}C & -1.612200 & 0.069342 & -0.340980\end{array}$ $\mathrm{H}-2.2914250 .485080 .415130$ $\mathrm{H}-1.699899-1.030196-0.386763$ C $-1.7202420 .535723-1.327711$ C $2.6701301 .333854-0.271598$ C $4.1093461 .585434-0.578814$ H $4.736260 \quad 0.731305-0.293880$ \begin{tabular}{l}
4.460545 \\
\hline
\end{tabular} H $4.2587131 .791662-1.651516$ H $2.755195 \quad 4.079212 \quad 0.511108$ H $2.7017114 .050315-1.262296$ H $1.243659 \quad 4.504539-0.326915$ C $-1.606189 \quad 3.880183 \quad 2.214835$ C $-2.552946 \quad 4.486946 \quad 1.389816$ $\begin{array}{lllll}\text { C }-2.507601 & 4.294391 & 0.012327\end{array}$ C $-1.508313 \quad 3.471891 \quad-0.568643$ C $-1.5083130 .47891-0.568643$ $\begin{array}{llll}C & -1.476651 & 3.248325 & -2.083228\end{array}$ $\begin{array}{llll}C & -2.883163 & 3.089315 & -2.674676\end{array}$ C $-0.718081-4.387659-2.802820$ $\mathrm{H}-0.9173302 .313843-2.259141$ $-3.4742802 .348316-2.112287$ $\mathrm{H}-2.8141792 .758850-3.724662$ H $-3.432394 \quad 4.045275 \quad-2.669414$ H $0.3300514 .452685-2.472006$ H $-0.723093 \quad 4.218418-3.893638$ H $-1.2020305 .359354-2.601623$ H $-3.254493 \quad 4.769658-0.625054$ $\begin{array}{lllll}\mathrm{H} & -3.335918 & 5.111994 & 1.825301\end{array}$ H $-1.649618 \quad 4.033409 \quad 3.296596$ $\begin{array}{llll}\text { C } 3.135214 & -1.127291 & -2.290423\end{array}$ C $4.366302-0.929348-3.184912$ $\begin{array}{llll}C & 2.174222 & -2.134902 & -2.949061\end{array}$ I $2.601248-0.168782-2.227734$ H $5.113997-0.262634-2.227734$ H $5.113997-0.262634-2.72531$ $4.863499-1.891319-3.394711$ $1.064335-0.498899-4.154702$ $\begin{array}{lllll}\mathrm{H} & 1.263092 & -2.270448 & -2.344650 \\ \mathrm{H} & 1.880461 & -1.783074 & -3.952578\end{array}$

H $2.658369-3.120558-3.057400$ 622392 H $5.264733-4.0837370 .618776$ H $4.270156-3.041328 \quad 2.638881$ 79

$28 \mathrm{R}=\mathrm{Me} \mathrm{ET} / \mathrm{pi}$

Ni $0.084349-0.054633-0.125855$ $\begin{array}{llll}\mathrm{N} & 0.341689 & 1.963447 & -0.123357\end{array}$ $\begin{array}{lllll}\text { C } & 1.589274 & 2.329661 & -0.239523\end{array}$ $\begin{array}{llll}\text { C } 2.069882 & 3.745223 & -0.193558\end{array}$ $\begin{array}{llll}\text { C } & -0.607923 & 2.886301 & 0.424449\end{array}$ $\begin{array}{llll}\text { C }-0.607923 & 2.886301 & 0.424449 \\ \text { C }-0.522016 & 3.131890 & 1.817960\end{array}$ $\begin{array}{llll}\text { C } & -0.522016 & 3.131890 & 1.817960 \\ \text { C } & 0.623092 & 2.640658 & 2.672984\end{array}$ C 0.6230922 .6406582 .672984 H 1.0271073 .5227233 .207233 H 1.4337502 .3035752 .019556 $\begin{array}{llll}\text { C } 0.360859 & 1.505020 & 3.695016\end{array}$ H $-0.098604 \quad 1.977426 \quad 4.585913$ B $-0.777993 \quad 0.411181 \quad 3.277413$ F $-0.618537-0.815050 \quad 3.948044$ $\begin{array}{llll}\text { F } & -0.690978 & 0.067672 & 1.785304\end{array}$ F $-2.070978 \quad 0.922368 \quad 3.442705$ C $1.719727 \quad 0.956609 \quad 4.210929$ H $2.260290 \quad 1.774764 \quad 4.724382$ H $1.499762 \quad 0.198463 \quad 4.981110$ $\begin{array}{lllll}\text { C } 2.708838 & 0.344115 & 3.196689\end{array}$ H $3.673970 \quad 0.173615 \quad 3.707033$ $\begin{array}{llll}\text { H } 3.673970 & 0.173615 & 3.707033\end{array}$ $\begin{array}{llll}H & 2.928405 & 1.066842 & 2.392269\end{array}$ $\begin{array}{llll}\text { C } 2.229015 & -0.980598 & 2.580126\end{array}$ H $2.168697-1.7396683 .376279$ $\begin{array}{llll}\text { H } 1.202010 & -0.838113 & 2.229684\end{array}$ C $3.055127-1.5416831 .449261$ $\begin{array}{llll}\text { C } 2.935918 & -1.068767 & 0.122525\end{array}$ $\begin{array}{llll}\text { N } 2.049236 & 0.039745 & -0.080772\end{array}$ $\begin{array}{llll}\text { C } 3.609537 & -1.678418 & -0.951233\end{array}$ $\begin{array}{llll}\text { C } 4.462721 & -2.749441 & -0.662687\end{array}$ $\begin{array}{llll}\text { C } 4.640533 & -3.199671 & 0.639929\end{array}$ $\begin{array}{lll}\text { C } 3.933605 & -2.602251 & 1.679929\end{array}$ $\begin{array}{llll}\text { C } 0.043615 & -2.005487 & -0.002428\end{array}$ $\begin{array}{llll}\text { H } & 0.931119 & -2.409535 & -0.506317\end{array}$ H $-0.874739-2.433508-0.424311$ H $0.074915 \quad-2.165575 \quad 1.084053$ C $-1.717829-0.068679-1.003782$ C $-0.708963-0.255214-1.0599476$ $\begin{array}{lllll}C & -0.708963 & -0.255214 & -1.959476\end{array}$ H $-2.1513300 .914025-0.810930$ $\mathrm{H}-2.306276-0.911536-0.636217$ H $-0.3466590 .579580-2.566087$ H $-0.516554-1.245924-2.376368$ $\begin{array}{llll}\text { C } 2.560366 & 1.231924 & -0.284665\end{array}$ $\begin{array}{llll}\text { C } 4.017920 & 1.538823 & -0.405994\end{array}$ H $4.634424 \quad 0.653625-0.210970$ $\begin{array}{lllll}\text { H } & 4.307361 & 2.329209 & 0.303874\end{array}$ H $4.2474221 .917417-1.416545$ H $2.691794 \quad 3.9229890 .699255$ H $2.695530 \quad 3.962829-1.074028$ H $1.226348 \quad 4.445390 \quad-0.160569$ $\begin{array}{llll}\text { C }-1.525467 & 3.906747 & 2.397269\end{array}$ C $-2.555291 \quad 4.465976 \quad 1.627962$ $\begin{array}{llll}C & -2.579318 & 4.255115 & 0.251403\end{array}$ C $-1.6150333 .458829-0.373959$ $\begin{array}{llll}C & -1.615033 & 3.458829 & -0.373959 \\ C & -1.607791 & 3.305997 & -1.885572\end{array}$ $\begin{array}{llll}C & -1.607791 & 3.305997 & -1.885572\end{array}$ $\begin{array}{llll}C & -3.006120 & 3.096915 & -2.484000\end{array}$ $\begin{array}{lllll}C & -0.922815 & 4.508084 & -2.557867\end{array}$ $\begin{array}{llll}\mathrm{H} & -1.004263 & 2.417964 & -2.120849\end{array}$ H $-3.5524032 .288704-1.976408$ $\begin{array}{llll}\mathrm{H} & -2.922218 & 2.840849 & -3.55164\end{array}$ H $-3.6115824 .014145 \quad-2.413928$ H $0.115875 \quad 4.626803 \quad-2.217001$ H $-0.912546 \quad 4.381378-3.652265$ н $-1.463787 \quad 5.438848 \quad-2.323432$ н $-3.365296 \quad 4.714995-0.348486$ H $-3.330103 \quad 5.069791 \quad 2.105651$ H $-1.500584 \quad 4.077370 \quad 3.473974$ $\begin{array}{llll}\text { C } 3.399024 & -1.257714 & -2.396817\end{array}$ $\begin{array}{llll}\text { C } 4.713784 & -0.878875 & -3.09484\end{array}$

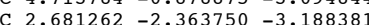
C $2.781262-2.363750-3.188381$ $5.262667-0.100523-2.403189$ H $5.262667-0.100523-2.545479$ $-5.37407-1.752626-3.187493$ $4.509575-0.507991-4.111397$ H $1.727910-2.641266-2.716763$ $\begin{array}{llll}\text { H } 2.477765 & -2.026701 & -4.21693\end{array}$ H $3.304328-3.270329-3.24505$ H $4.993793-3.242579-1.47770$ H $5.314656-4.032266 \quad 0.843057$ H $4.043175-2.9760002 .699637$ 
H $-0.063805 \quad 2.002341 \quad 4.581694$ B $-0.777810 \quad 0.428993 \quad 3.296140$ F $-0.567990 \quad-0.856596 \quad 3.81983$ F $-0.828310 \quad 0.236248 \quad 1.751476$ F $-2.056203 \quad 0.900744 \quad 3.596967$ C $1.735833 \quad 0.946550 \quad 4.218025$ H $2.278805 \quad 1.760573 \quad 4.734613$ H $1.5112620 .187178 \quad 4.985862$ $\begin{array}{lllll}\text { C } 2.719872 & 0.332513 & 3.202261\end{array}$ $c$
$\mathrm{H}$ $\begin{array}{llll}\text { H } & 3.675520 & 0.127104 & 3.71705\end{array}$ $\begin{array}{lllll}\text { H } & 2.965117 & 1.063349 & 2.412123\end{array}$ $\begin{array}{llll}\text { C } 2.208148 & -0.966241 & 2.560601 \\ \text { H } 2.117257 & -1.737820 & 3.341346\end{array}$ H $2.117257-1.7378203 .341346$

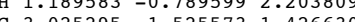
C $3.025295-1.525573-1.426630$ $\begin{array}{llll}\text { C } 2.888921-1.049153 & 0.102124\end{array}$ N $1.9950640 .056053 \quad-0.075896$ C $3.553534-1.660578-0.97968$ $\begin{array}{llll}\text { C } 4.415344 & -2.725807 & -0.69562\end{array}$ $\begin{array}{llll}\text { C } 4.611374 & -3.172919 & 0.605525 \\ \text { C } 3.911207 & -2.580103 & 1.652081\end{array}$ C $3.911207-2.580103 \quad 1.652081$ C $-0.117946-1.978879 \quad 0.221006$ H $0.844677-2.421008-0.069337$ H $-0.946244-2.437690-0.335225$ H $-0.304512-2.033415 \quad 1.301488$ C $-1.675078-0.079982-1.135285$ C $-0.560281-0.395348-1.955409$ H $-2.110025 \quad 0.917971-1.132627$ $\begin{array}{llll}\text { H } & -2.110025 & 0.917971 & -1.132627 \\ \text { H } & -2.335556 & -0.887021 & -0.816226\end{array}$ $\begin{array}{llll}\mathrm{H} & -2.335556 & -0.887021 & -0.816226 \\ \mathrm{H} & -0.390285 & -1.438821 & -2.234662\end{array}$ C $0.091303 \quad 0.521348-2.830866$ N $0.6960043 .229733-3.539035$ C $2.4703621 .232515-0.399578$ C $3.9058521 .540514-0.670250$ H $4.5521000 .684933-0.442257$ \begin{tabular}{l}
$4.2367142 .402422-0.071809$ \\
\hline
\end{tabular} H $4.036586 \quad 1.815024-1.730719$ H $2.664159 \quad 3.942724 \quad 0.497854$ H $2.534736 \quad 3.942196 \quad-1.274614$ H $1.139368 \quad 4.450651 \quad-0.265987$ C $-1.477326 \quad 3.922856 \quad 2.489307$ $\begin{array}{llll}C & -2.530585 & 4.489958 & 1.785076\end{array}$ C $-2.617952 \quad 4.3052840 .400151$ $\begin{array}{lllll}C & -2.617952 & 4.305284 & 0.400151\end{array}$ $\begin{array}{llll}C & -1.689372 & 3.514974 & -0.283895\end{array}$ C $-1.75522953 .407917-1.793990$ $\begin{array}{llll}C & -3.191665 & 3.149293 & -2.321032\end{array}$ $\begin{array}{llll}C & -1.195564 & 4.666935 & -2.475815\end{array}$ $\mathrm{H}-1.1178832 .571762-2.110118$ $\mathrm{H}-3.6868212 .321383-1.787605$ H $-3.1546732 .904528-3.395324$ H $-3.821502 \quad 4.046912-2.205617$ H $-0.145366 \quad 4.847789-2.198639$ H $-1.241050 \quad 4.555454-3.572074$ H $-1.782137 \quad 5.556645-2.188479$ H $-3.4253494 .787719-0.154598$ $\mathrm{H}-3.280101 \quad 5.092134 \quad 2.303981$ H $-1.396357 \quad 4.076565 \quad 3.566089$ $\begin{array}{llll}\text { C } 3.306384 & -1.269412 & -2.424061\end{array}$ C $2.512540-2.364553-3.208067$ C $2.512540-2.364553-3$. $\begin{array}{llll}\text { H } 2.701290 & -0.353463 & -2.44914\end{array}$ H $5.247778-0.220025-2.692978$ H $5.229709-1.886656-3.327462$ H $4.379935-0.593380-4.213838$ H $1.589963 \quad-2.619044-2.611672$ H $2.244137-2.028920-4.167746$ H $3.108172-3.287803-3.240879$ H $4.938666-3.217946-1.51593$ $\begin{array}{llll}\text { H } 5.293223 & -4.000921 & 0.801727\end{array}$ $\begin{array}{llll}\text { H } & 4.031942 & -2.951823 & 2.671207\end{array}$

$28 \mathrm{R}=\mathrm{Me} \mathrm{AC} / \mathrm{N}$

Ni $0.135485-0.090577-0.537162$ N $1.977101-0.009918-0.141949$ $\begin{array}{llll}\text { N } 0.284008 & 1.861218 & -0.279561\end{array}$ $\begin{array}{llll}\text { C } 2.493607 & 1.200938 & -0.063786\end{array}$ $\begin{array}{llll}\text { C } & 1.511462 & 2.281836 & -0.135430\end{array}$ $\begin{array}{llll}\text { C } 2.826173 & -1.119207 & 0.217894\end{array}$ C -0.8210192 .7035130 .048138$ C 3.9295791 .5140180 .196870 $\begin{array}{llll}\text { C } & 1.917398 & 3.702783 & 0.079092 \\ \text { C } & 0.113142 & -2.012249 & -0.849923\end{array}$ $\begin{array}{llll}\text { C } 0.113142 & -2.012249 & -0.849923\end{array}$ H $-0.686692-2.378855-0.188691$ H $1.081292-2.454285-0.584815$ H $-0.138151-2.192972-1.904880$ $\mathrm{N}-1.580909-0.077202-1.160715$ C $-2.743885-0.065919-1.152778$ $\begin{array}{llll}\text { C } & -4.152845 & -0.008842 & -1.077695\end{array}$ $\begin{array}{lllll}\text { C }-4.752074 & 0.344946 & 0.071282\end{array}$ H $-4.709594-0.279383-1.977760$ H $-5.840567 \quad 0.377634 \quad 0.121392$ H $-4.168105 \quad 0.584559 \quad 0.967679$ $\begin{array}{llll}\text { H } & 4.510322 & 0.614295 & 0.429884\end{array}$ \begin{tabular}{llll}
$\mathrm{H}$ & 4.510322 & 0.614295 & 0.429884 \\
\hline
\end{tabular} $\begin{array}{lllll}\text { H } & 4.371526 & 2.003601 & -0.688293\end{array}$ $\begin{array}{lllll}\text { H } & 4.371526 & 2.003601 & -0.688293 \\ \text { H } & 2.340961 & 3.844781 & 1.087345\end{array}$ H $2.6968793 .992509-0.643874$
H $1.056072 \quad 4.375047 \quad-0.021454$ C $-1.070465 \quad 2.941246 \quad 1.420160$ C $-2.223604 \quad 3.6626601 .741859$ $\begin{array}{lllll}\text { C }-3.077306 & 4.145089 & 0.757961\end{array}$ C $-2.795375 \quad 3.913909-0.587114$ C $-1.669046 \quad 3.182199 \quad-0.968763$ $\begin{array}{llll}\text { C }-1.317860 & 2.965172 & -2.430754\end{array}$ $\begin{array}{llll}C & -2.542742 & 2.890640 & -3.345626\end{array}$ C $-0.353688 \quad 4.051598 \quad-2.936238$ H $-0.7909371 .999562-2.497369$ H $-3.2900312 .178307-2.965824$ $\mathrm{H}-3.2900312 .178307-2.965824$ $\mathrm{H}-2.2379522 .571385-4.353885$ H $-3.030284-3.872751-3.447776$ H $0.585650-4.057638-2.365733$ H $-0.106083 \quad 3.882162-3.996340$ H $-0.815430 \quad 5.047964-2.842577$ H $-3.468283 \quad 4.304450-1.34984$ H $-3.969996 \quad 4.705248 \quad 1.038285$ н $-2.451404 \quad 3.833512 \quad 2.794862$ $\begin{array}{llll}\text { C } 2.701464 & -1.612728 & 1.534466\end{array}$ C $3.574646-2.6348371 .920670$ $\begin{array}{llll}\text { C } 4.508790 & -3.168599 & 1.038236\end{array}$ $\begin{array}{llll}\text { C } 4.561272 & -2.699097 & -0.285972\end{array}$ $\begin{array}{llll}\text { C } 3.716540 & -1.671415 & -0.718133\end{array}$ C $3.737597-1.230377-2.169182$ C $5.131491-0.749709-2.607182$ C $3.246897-2.359602-3.089368$ $\begin{array}{llll}C & 3.246897 & -2.359602 & -3.089368\end{array}$ $\begin{array}{llll}\text { H } 3.036178 & -0.389678 & -2.279175\end{array}$ H $5.5123350 .053272-1.956103$ H $5.860963-1.576669-2.581996$ $\mathrm{H} 5.094365-0.370200-3.642079$ H $2.242647-2.699433-2.79895$ H $3.210145-2.010639-4.133577$ H $3.926294-3.226068-3.04252$ H $5.268799-3.149801-0.986419$ H $5.183016-3.9661631 .359306$ $\begin{array}{llll}\text { H } 3.498752 & -3.026882 & 2.937085\end{array}$ C $-0.133195 \quad 2.488844 \quad 2.516890$ H $0.837328 \quad 2.254030 \quad 2.068797$ H $0.052625 \quad 3.368145 \quad 3.163032$ C $-0.530381 \quad 1.278002 \quad 3.412901$ н $-1.123090 \quad 1.691607 \quad 4.252773$ B $-1.569166 \quad 0.1740772 .797593$ B -1.5691660 .1740772 .797593$ $\begin{array}{llll}\mathrm{F}-1.164095 & -0.339514 & 1.486092\end{array}$ $\begin{array}{llll}F & -1.672475 & -0.936534 & 3.666531 \\ F & -2.863870 & 0.762537 & 2.609463\end{array}$ $\begin{array}{llll}\text { F } & -2.863870 & 0.762537 & 2.609463 \\ \text { C } & 0.745050 & 0.709289 & 4.098997\end{array}$ $\begin{array}{llll}\text { C } & 0.745050 & 0.709289 & 4.098997 \\ \text { H } & 0.419024 & -0.101342 & 4.772098\end{array}$ $\begin{array}{llll}\text { H } & 0.419024 & -0.101342 & 4.772098\end{array}$ $\begin{array}{llll}\text { H } & 1.179426 & 1.495326 & 4.746869\end{array}$ C $1.9077510 .183995 \quad 3.226885$ H $2.213536 \quad 0.956080 \quad 2.500534$ H $2.7881390 .037696 \quad 3.879535$ C $1.626515-1.135201 \quad 2.483185$ H $1.455975-1.927092 \quad 3.230800$ H $0.680162-1.038154 \quad 1.932468$

$28 \mathrm{R}=$ CHCNEt $\mathrm{ET} / \mathrm{p}$

Ni $0.020196 \quad 0.031807 \quad-0.348464$ $\begin{array}{lllll}\mathrm{N} 2 & 2.015006 & 0.057404 & -0.241291\end{array}$ C $2.904413-1.062299-0.063756$ C $2.550300 \quad 1.253791-0.281661$ $\begin{array}{llll}\text { C } & 2.550300 & 1.253791 & -0.281661 \\ \text { C } & 1.013245 & 1.560390 & -0.220149\end{array}$

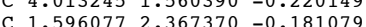
C 2.1039763 .7616540 .002738 $\begin{array}{llll}\text { C } & 2.103976 & 3.761654 & 0.002738 \\ N & 0.337267 & 2.029296 & -0.144897\end{array}$ $\begin{array}{lllr}\text { N } & 0.337267 & 2.029296 & -0.144897 \\ \text { C } & -0.0 .604710 & 2.925033 & 0.466914\end{array}$ $\begin{array}{llll}\text { C } & -0.604710 & 2.925033 & 0.466914 \\ \text { C }-0.146946 & -1.982157 & -0.268972\end{array}$ $\begin{array}{lllll}\text { H } & 0.730993 & -2.175172 & 0.368082\end{array}$ $\begin{array}{llll}\text { C }-1.420377 & -2.508104 & 0.413177\end{array}$ $\begin{array}{llll}\text { C }-1.204685 & -3.932541 & 0.940009\end{array}$ H $-1.679371-1.844962 \quad 1.246256$ $\mathrm{H}-2.254675-2.512194-0.304363$ $\mathrm{H}-0.415810-3.946343 \quad 1.706671$ н $-2.131582-4.301845 \quad 1.403831$ H $-0.921626-4.622187-0.130241$ H $-0.921626-4.6221870 .130241$ C $0.054094-2.617125-1.537498$ $\mathrm{N} 0.133317-3.139822-2.581922$ C $-1.7352670 .210601-1.330137$ C $-0.6760380 .179980-2.244256$ H $-2.1585051 .147637-0.962414$ H $-2.367191-0.667530-1.194090$ H $-0.251241 \quad 1.101165-2.650584$ Н $-0.470123-0.722604-2.823254$ H $4.627508 \quad 0.654607 \quad-0.259280$ H $4.243821 \quad 2.088541 \quad 0.720599$ H $4.3046642 .235406 \quad-1.039920$ H $2.7464313 .837875 \quad 0.894383$ H $2.718341 \quad 4.053259 \quad-0.864636$ H $1.275608 \quad 4.470472 \quad 0.117755$ C $-0.545537 \quad 3.032420 \quad 1.878798$ C $-1.556899 \quad 3.784736 \quad 2.518355$ $-2.555952 \quad 4.43$ $\begin{array}{lllll}C & -2.555952 & 4.437080 & 1.773741\end{array}$ $\begin{array}{llll}C-2.531191 & 4.367717 & 0.377708\end{array}$ $\begin{array}{llll}C & -1.565837 & 3.616754 & -0.296616\end{array}$ C $-1.4962983 .670250-1.813588$ $\begin{array}{llll}\text { C } & -2.861142 & 3.523830 & -2.496321 \\ \text { C }-0.819130 & 4.973591 & -2.274853\end{array}$

H $-0.860432 \quad 2.838824-2.145810$ H $-3.4004392 .630334-2.15187$ H $-2.727492 \quad 3.447033-3.586148$ H $-3.4980604 .401200 \quad-2.306903$ H $0.194251 \quad 5.076362-1.860544$ H $-0.746136 \quad 5.000027-3.373642$ н $-1.406183 \quad 5.846639-1.947501$ H $-3.280613 \quad 4.913995-0.196857$ H $-3.334520 \quad 5.0100492 .283497$ H -1.5539543 .8495463 .609002$ $\begin{array}{llll}\mathrm{H}-1.553954 & 3.849546 & 3.609002\end{array}$ $\begin{array}{llll}C & 3.035015 & -1.541575 & 1.258519\end{array}$ $\begin{array}{llll}\text { C } 3.976864 & -2.558827 & 1.492800\end{array}$ C $4.731529-3.0957370 .456709$ $\begin{array}{llll}\text { C } 4.518245 & -2.655036 & -0.854944\end{array}$ C $3.597607-1.641388-1.146434$ $\begin{array}{llll}\text { C } 3.364442 & -1.229228 & -2.593148\end{array}$ $\begin{array}{llll}\text { C } 4.475897 & -0.321908 & -3.14350\end{array}$ $\begin{array}{llll}\text { C } 3.227689 & -2.447260 & -3.524499\end{array}$ H $2.413441-0.674317-2.62903$ H $4.5620950 .627988-2.600752$ H $5.451153-0.833345-3.088072$ H $4.275802-0.090000-4.201730$ H $2.489814-3.168621-3.145047$ H $2.894730-2.111825-4.520893$ н $4.197364-2.956469-3.656099$ H $5.072907-3.122600-1.670928$ H $5.0761531-3.883982-1.670928$ $\begin{array}{llll}H & 5.461531 & -3.883982 & 0.655452\end{array}$ $\begin{array}{llll}H & 4.091653 & -2.937166 & 2.511929\end{array}$ c 0.5743362 .4567652 .711530 H 1.3731032 .1191952 .044229 H 1.0095383 .2958293 .289402 $\begin{array}{llll}\text { C } 0.265444 & 1.282420 & 3.671189\end{array}$ $\begin{array}{llll}\text { H } & -0.225216 & 1.711593 & 4.566855\end{array}$ $\begin{array}{lllll}\text { B }-0.849448 & 0.230007 & 3.117854\end{array}$ F $-0.686608-1.0779313 .606594$ $\begin{array}{llll}F & -0.717651 & 0.108482 & 1.584226\end{array}$ $\begin{array}{lllll}\text { F } & -2.155170 & 0.685887 & 3.313749\end{array}$ C $1.599565 \quad 0.688180 \quad 4.200358$ H $1.347296-0.135085 \quad 4.889514$ H $2.115484 \quad 1.455848 \quad 4.807668$ C $2.631154 \quad 0.166375 \quad 3.178025$ н $2.917195 \quad 0.9701702 .478420$ H $3.559450-0.0926683 .718158$ $\begin{array}{llll}\text { H } 3.559450 & -0.092668 & 3.718158 \\ \text { C } & 2.152491 & -1.060359 & 2.384558\end{array}$ $\begin{array}{llll}\text { C } 2.152491 & -1.060359 & 2.384558\end{array}$ $\begin{array}{llll}\text { H } & 2.008396 & -1.895309 & 3.089098 \\ \text { H } & 1.157829 & -0.837181 & 1.986267\end{array}$ 87

$28 \mathrm{R}=\mathrm{CHCNEt} \mathrm{AC} / \mathrm{pi}$

$\mathrm{Ni}-0.059525-0.010211-0.30817$ $\begin{array}{lllll}N & 1.951255 & 0.032967 & -0.257003\end{array}$ $\begin{array}{llll}\text { C } 2.858705 & -1.073907 & -0.09247\end{array}$ $\begin{array}{llll}\text { C } 2.459019 & 1.232510 & -0.366285\end{array}$ $\begin{array}{llll}\text { C } 3.916704 & 1.564402 & -0.342808\end{array}$ $\begin{array}{llll}\text { C } 1.490311 & 2.344252 & -0.270183\end{array}$ $\begin{array}{llll}\text { C } 1.992244 & 3.747294 & -0.177653\end{array}$ N $0.240799 \quad 2.001343 \quad-0.154497$ C $-0.671681 \quad 2.905053 \quad 0.495883$ C $-0.240047-2.020829-0.098011$ H $0.547837 \quad-2.138859 \quad 0.660805$ $\begin{array}{llll}\text { C } & -1.594618 & -2.482187 & 0.455193\end{array}$ $\begin{array}{llll}C & -1.594618 & -2.482187 & 0.455193\end{array}$ C $-1.473759-3.8750761 .088552$ - $1.932912-1.7627031 .210502$ $\begin{array}{llll}\mathrm{H} & -2.336429 & -2.522276 & -0.357199\end{array}$ H $-0.782809-3.8509601 .943789$ $\begin{array}{llll}\text { H } & -2.457627 & -4.202231 & 1.456396\end{array}$ $\begin{array}{llll}\text { H }-1.111418 & -4.615252 & 0.359174\end{array}$ $\begin{array}{llll}\text { C } 0.110904 & -2.758978 & -1.273678\end{array}$ $\begin{array}{llll}\mathrm{N} & 0.286547 & -3.370374 & -2.256331\end{array}$ $\begin{array}{lllll}\text { C }-1.743423 & 0.178916 & -1.364891\end{array}$ $\begin{array}{llll}\text { C }-0.655837 & -0.063722 & -2.237816\end{array}$ H $-2.1232321 .182186-1.181481$ H $-2.444790-0.633428-1.179255$ H $-0.542728-1.063105-2.671886$ C $0.061954 \quad 0.924740-2.971449$ H $4.5467920 .671158-0.403579$ H 4.1544622 .0851120 .601023 $\begin{array}{llll}\text { H } & 4.154462 & 2.085112 & 0.601023 \\ \text { H } & .174580 & 2.254609 & -1.159689\end{array}$ $\begin{array}{llll}\text { H } & 4.174580 & 2.254609 & -1.159689 \\ \text { H } & 2.709772 & 3.866284 & 0.648952\end{array}$ H $2.5203804 .002660-1.111152$ $\begin{array}{llll}\mathrm{H} & 2.520380 & 4.002660 & -1.111152 \\ \mathrm{H} & 1.166849 & 4.451505 & -0.023736\end{array}$ C $-0.528954 \quad 3.020222 \quad 1.900854$ $\begin{array}{llll}\text { C } & -0.528954 & 3.020222 & 1.900854 \\ \text { C } & -1.489503 & 3.788177 & 2.600015\end{array}$ $\begin{array}{llll}\text { C }-1.489503 & 3.788177 & 2.600015 \\ \text { C }-2.527169 & 4.445320 & 1.918536\end{array}$ C $-2.589565 \quad 4.363444 \quad 0.513892$ C $-1.6709853 .603587-0.213932$ C $-1.7003653 .662933-1.731192$ $\begin{array}{llll}\text { C } & -3.103310 & 3.472414 & -2.320792\end{array}$ $\begin{array}{llll}\text { C }-1.106122 & 4.990279 & -2.235375\end{array}$ $\mathrm{H}-1.051326 \quad 2.867967 \quad-2.118811$ 
H $-1.413211 \quad 3.861380 \quad 3.687615$ C $3.031547-1.5371$ $\begin{array}{llll}\text { C } 3.989715 & -2.546264 & 1.450730\end{array}$ $\begin{array}{llll}\text { C } 4.720805 & -3.083298 & 0.398765\end{array}$ $\begin{array}{llll}\text { C } 4.468846 & -2.652967 & -0.909038\end{array}$ C $3.530981-1.653635-1.189460$ C $3.254521-1.268403-2.636268$ C $4.290577-0.295796-3.218716$ $\begin{array}{llll}C & 3.184831-2.506778 & -3.555711\end{array}$ H $2.272210-0.773868-2.66$ $\begin{array}{llll}\text { H } 2.272210 & -0.773868 & -2.668615\end{array}$ $\begin{array}{lllll}\mathrm{H} & 4.285133 & 0.681635 & -2.722438\end{array}$ $\begin{array}{llll}\text { H } 5.305540 & -0.718807 & -3.13828\end{array}$ H $4.073144-0.124109-4.28405$ H $2.524531-3.282626-3.14214$ H $2.789985-2.205273-4.540025$ H $4.188475-2.933229-3.72182$ H $5.005530-3.124006-1.734768$ H $5.461757 \quad-3.864967 \quad 0.582486$ $\begin{array}{llll}\text { H } & 4.133599 & -2.913689 & 2.470301\end{array}$ C $0.624662 \quad 2.433696 \quad 2.676078$ H $1.383074 \quad 2.072554 \quad 1.974462$ H $1.104455 \quad 3.268501 \quad 3.223806$ $\begin{array}{lllll}\text { C } & 0.331924 & 1.280680 & 3.665172\end{array}$ H $-0.144662 \quad 1.732501 \quad 4.557478$ B $-0.800210 \quad 0.221467 \quad 3.160897$ F $-0.611510-1.0904913 .616839$ F $-0.747010 \quad 0.111347 \quad 1.596381$ F -0.7470100 .1113471 .596381$ $\begin{array}{lllll}\mathrm{F} & -2.097925 & 0.665823 & 3.403139\end{array}$ C 1.6741800 .7011354 .190013 $\begin{array}{llll}\text { H } & 1.435508 & -0.114975 & 4.892427\end{array}$ H 2.1898971 .4806234 .781607 $\begin{array}{llll}\text { C } 2.694192 & 0.173676 & 3.160635\end{array}$ H $2.980590 \quad 0.974757 \quad 2.458033$ H $3.624751-0.096615 \quad 3.690648$ $\begin{array}{llll}\text { C } 2.184432 & -1.044080 & 2.375186\end{array}$ H $2.039107-1.877532 \quad 3.081278$ H $1.186674-0.798274 \quad 2.001107$

$28 \mathrm{R}=\mathrm{CHCNE} \mathrm{AC} / \mathrm{N}$

Ni $0.129468 \quad 0.059869 \quad-0.292529$ N $2.032460 \quad 0.040266-0.228213$ C 2.880717 C.607636 $1.223815-0.22$ $\begin{array}{lll}\text { C } 2.607636 & 1.223815 & -0.221230\end{array}$ (.07502 C 1.6751432 .34 C $2.1721753 .752218-0.030458$ $\begin{array}{llll}\mathrm{N} & 0.426326 & 1.982972 & -0.034072\end{array}$ $\begin{array}{lllll}C & -0.557816 & 2.886738 & 0.470804\end{array}$ C $-0.005906-1.852673-0.804449$ $\begin{array}{llll}\text { H } & 1.002286 & -2.257835 & -0.619347\end{array}$ C $-1.039306-2.678195 \quad-0.029553$ C $-0.861918-4.177898 \quad-0.288403$ н $-0.925680 \quad-2.456986 \quad 1.042343$ H $-2.054617-2.371910 \quad-0.316898$ H $0.135651-4.519063 \quad 0.028279$ H $-1.612290 \quad-4.753945 \quad 0.274206$ H $-0.981166-4.410583-1.358175$ C $-0.237487-1.778268-2.220059$ $\begin{array}{llll}\text { C } & -0.237487 & -1.778268 & -2.220059 \\ \mathrm{~N} & -0.433834 & -1.686234 & -3.371726\end{array}$ $\begin{array}{llll}\mathrm{N} & -0.433834 & -1.686234 & -3.371726 \\ \mathrm{~N} & -1.687279 & 0.204101 & -0.497480\end{array}$ $\begin{array}{llll}\mathrm{N} & -1.687279 & 0.204101 & -0.497480 \\ \mathrm{C} & -2.821913 & 0.327493 & -0.279641\end{array}$ $\begin{array}{llll}\text { C }-4.188866 & 0.484561 & 0.037236\end{array}$ $\begin{array}{llll}\text { C }-4.559784 & 0.866143 & 1.270531\end{array}$ $\mathrm{H}-4.906045 \quad 0.267511-0.75806$ H $\quad \begin{array}{llll}-5.618873 & 0.976388 & 1.503397\end{array}$ H $-3.816234 \quad 1.052356 \quad 2.053528$ $\begin{array}{llll}\text { H } & 4.656299 & 0.552552 & -0.105218\end{array}$ H 4.3177062 .0926950 .727259 H $4.3925152 .058203-1.040073$ H $2.790016 \quad 3.889819 \quad 0.872607$ H $2.8082613 .993548 \quad-0.897656$ H $1.337298 \quad 4.462258 \quad 0.019883$ C $-0.532480 \quad 3.153867 \quad 1.858185$ C $-1.561909 \quad 3.9480702 .371117$ C $-2.555733 \quad 4.465663 \quad 1.548563$ C $-2.555733 \quad 4.465663 \quad 1.548563$ $\begin{array}{lllll}C & -2.541494 & 4.199966 & 0.178993\end{array}$ C $-1.5470483 .400354-0.386877$ C $-1.4889823 .143031-1.884512$ C $-2.8645453 .142440-2.554661$ C $-0.5668814 .151583-2.589914$ H $-1.0491882 .142712-2.027756$ H $-3.5793142 .501455-2.01780$ H $-2.7756232 .771412-3.586649$ H $-3.289744 \quad 4.157277-2.604664$ H $0.462344 \quad 4.099379 \quad-2.208464$ H $-0.536848 \quad 3.945372 \quad-3.671541$ H $-0.9351435 .179716-2.441485$ H $-3.319903 \quad 4.622025-0.454645$ H $-3.345575 \quad 5.084968 \quad 1.972940$ H $-1.579170 \quad 4.151010 \quad 3.441979$ C 2.971485 $\begin{array}{llll}\text { C } 2.971485 & -1.575450 & 1.329494 \\ \text { C } 3.856737 & -2.625378 & 1.582826\end{array}$ $\begin{array}{llll}C & 3.856737 & -2.625378 & 1.582826\end{array}$ $\begin{array}{llll}\text { C } & 4.605519 & -3.208082 & 0.565396 \\ \text { C } & .444616 & -2.765057 & -0.756321\end{array}$ $\begin{array}{llll}\text { C } & 4.444616 & -2.765057 & -0.756321 \\ \text { C } 3.569944 & -1.715594 & -1.065882\end{array}$ C $3.364478-1.305164-2.511856$ $\begin{array}{llll}\text { C } 4.613214 & -0.600151 & -3.105797\end{array}$ C $3.006968-2.504735-3.4$ $\begin{array}{llll}\text { н } 2.521756 & -0.597678 & -2.550873\end{array}$ $\begin{array}{lllll}\text { H } & 4.892787 & 0.303407 & -2.543146\end{array}$ H $5.479306-1.284153-3.100923$ H $4.413493-0.308911-4.151249$ н $2.162992-3.080622-2.992442$ $\begin{array}{lllll}\text { H } 2.713826 & -2.151006 & -4.402658\end{array}$ H $3.868223-3.184097-3.516777$ H $5.998304-3.255752-1.558829$ $\begin{array}{llll}H & 5.295633 & -4.027086 & 0.784013\end{array}$ $\begin{array}{lllllll}H & 3.942669 & -2.999041 & 2.606542\end{array}$ $\begin{array}{llll}C & 0.5646682 .661688 & 2.776577\end{array}$ H 1.4319722 .3895112 .166061 H 0.8959973 .5317743 .374202 C $0.282456 \quad 1.467409 \quad 3.736262$ $\begin{array}{lllll}\text { H } & -0.131195 & 1.906847 & 4.665689\end{array}$ B $-0.8988390 .410965 \quad 3.331440$ F $-0.901929-0.684550 \quad 4.225576$ F $-0.751736-0.142240 \quad 1.980029$ F $-2.174421 \quad 1.062942 \quad 3.357415$ C $1.6329880 .840952 \quad 4.186453$ H $1.397834 \quad 0.047442 \quad 4.915491$ H $2.214827 \quad 1.606129 \quad 4.736151$ C $2.589272 \quad 0.259951 \quad 3.120946$ \begin{tabular}{l}
$2.798113 \quad 1.020342 \quad 2.349805$ \\
\hline
\end{tabular} $\begin{array}{lll}\text { H } 2.798113 & 1.020342 & 2.349805\end{array}$ C 3.5643980 .0619363 .602727 $\begin{array}{llll}\text { C } 2.103783 & -1.034147 & 2.443633\end{array}$ $\begin{array}{llll}\text { H } 2.012577 & -1.817008 & 3.214518\end{array}$ 85

$28 \mathrm{R}=\mathrm{n}-\mathrm{Pr} \mathrm{ET} / \mathrm{p}$

Ni $0.072511-0.111936-0.170446$ N $2.156441-0.051244-0.032245$ $\begin{array}{llll}\text { C } 3.077781-1.130278 & 0.205602\end{array}$ $\begin{array}{llll}\text { C } 2.635737 & 1.150234 & -0.217203\end{array}$ $\begin{array}{llll}\text { C } 4.086544 & 1.513037 & -0.246544\end{array}$ $\begin{array}{llll}\text { C } 1.637825 & 2.224216 & -0.244050\end{array}$ $\begin{array}{llll}\text { C } 2.090982 & 3.650953 & -0.236846\end{array}$ N $0.387666 \quad 1.838871 \quad-0.157177$ C $-0.571513 \quad 2.808552 \quad 0.288464$ $\begin{array}{lllll}\text { C }-1.888963 & 0.114042 & -0.376033\end{array}$ H -2.0337840 .8404680 .435786$ C $-2.783607-1.085318-0.167555$ $\begin{array}{ll}C & -2.783607-1.085318-0.167555 \\ C & -4.251066-0.629400-0.080287\end{array}$ $\begin{array}{llll}C & -4.251066 & -0.629400 & -0.080287\end{array}$ $\mathrm{H}-2.691153-1.815567-0.988881$ $\mathrm{H}-2.518136-1.5869950 .775373$ H $-4.568697-0.126220-1.006756$ H $-4.911132-1.4956500 .081423$ H -4.3850470 .0689350 .758439$ H $-2.0380510 .577958-1.360869$ $\begin{array}{llll}\text { C } 0.089444 & -2.033635 & -0.771780\end{array}$ C $0.026312-1.175650-1.87805$ H $-0.797709-2.558586-0.418061$ н $1.043924-2.458563-0.445514$ H $-0.912857-1.038629-2.418878$ н $0.923430-0.924080-2.447214$ H $4.724440 \quad 0.650791-0.021251$ $\begin{array}{llll}4.297998 & 2.306244 & 0.4874\end{array}$ H $4.3609691 .914432 \quad-1.236252$ 12.6539543 .8844600 .682563 $1.7363653 .840799-1.086416$ H $1.2384554 .338404-0.288207$ C $-0.565453 \quad 3.1057181 .672148$ $\begin{array}{llll}C-1.564256 & 3.958757 & 2.146373\end{array}$ C $-2.511233 \quad 4.511766 \quad 1.292934$ $\begin{array}{llll}C & -2.469783 & 4.230308 & -0.069417\end{array}$ c $-1.504073 \quad 3.370772-0.599100$ $\begin{array}{llll}\text { C }-1.428220 & 3.111347 & -2.094449\end{array}$ $\begin{array}{llll}\text { C } & -2.808163 & 2.949638 & -2.742366\end{array}$ C $-0.641292 \quad 4.220771-2.813426$ H $-0.871552 \quad 2.170978-2.233786$ $\mathrm{H}-3.429490 \quad 2.226994 \quad-2.194268$ H $-2.6980292 .602842 \quad-3.78145$ H $-3.3497193 .907708-2.775342$ - $-3.3497193 .907708-2.775342$ H $0.390398-4.297201-2.441750$ $\mathrm{H}-0.599768 \quad 4.019758-3.895902$ $\mathrm{H}-1.1292025 .197402-2.663168$ $\mathrm{H}-3.206303 \quad 4.684452-0.732267$ H -3.2822175 .1735931 .688582$ H -1.5903374 .1889113 .212647$ C $3.186579-1.6032851 .536109$ $\begin{array}{llll}\text { C } 4.109063 & -2.631781 & 1.790401\end{array}$ $\begin{array}{llll}\text { C } 4.874700 & -3.197603 & 0.777298\end{array}$ $\begin{array}{llll}\text { C } 4.722185 & -2.736305 & -0.531823\end{array}$ $\begin{array}{llll}\text { C } 3.833867 & -1.699497 & -0.842252\end{array}$ $\begin{array}{llll}\text { C } 3.711545 & -1.266957 & -2.293069\end{array}$ $\begin{array}{llll}\text { C } 5.066913 & -0.757195 & -2.874132\end{array}$ $\begin{array}{llll}\text { C } 3.190369 & -2.408696 & -3.179138\end{array}$ H $2.996342-0.433552-2.349310$ H $5.5030980 .046600-2.262463$ H $5.799564-1.579288-2.924587$ $5.799564-1.579288-2.924587$ $1.913589-0.377249-3.897853$ - $2.240343-2.816043-2.803032$ - $3.033961-2.051097-4.210117$ H $5.309625-3.192847-1.331712$

H $5.579608 \quad-4.0024920 .998368$ $\begin{array}{llll}\text { H } & 4.204358 & -2.996127 & 2.816247\end{array}$ C $0.504102 \quad 2.603063 \quad 2.617612$ H $1.394718 \quad 2.3481132 .033016$ н $0.808344 \quad 3.466328 \quad 3.239267$ C $0.207218 \quad 1.401564 \quad 3.557826$ H $-0.335750 \quad 1.822099 \quad 4.428832$ B $-0.8543110 .265543 \quad 3.078300$ F $-0.957119-0.7586934 .033561$ F $-0.407203-0.464561 \quad 1.812100$ $\begin{array}{llll}\text { F } & -2.114363 & 0.809316 & 2.768607\end{array}$ C $1.545162 \quad 0.881565 \quad 4.155791$ H 1.2979020 .1089974 .903187 H 2.0234621 .7093254 .713300 C 2.6182010 .3171103 .197006 H $2.764379 \quad 1.0081822 .352319$ н $3.5870260 .296949 \quad 3.728250$ C $2.322877-1.098524 \quad 2.665341$ H $2.409622-1.8095403 .502722$ H $1.277408-1.1416092 .338843$ 86

$28 \mathrm{R}=\mathrm{n}-\mathrm{Pr} \mathrm{AC} / \mathrm{pi}$

Ni $0.112426-0.166912-0.226268$ N $2.116074 \quad 0.018191 \quad-0.091647$ C $3.044725-1.049667 \quad 0.130869$ $\begin{array}{llll}C & 2.559073 & 1.215738 & -0.374864\end{array}$ $\begin{array}{llll}\text { C } 2.559073 & 1.215738 & -0.374864 \\ \text { C } 3.993151 & 1.581366 & -0.573327\end{array}$ $\begin{array}{llll}C & 3.993151 & 1.581366 & -0.573327\end{array}$ $\begin{array}{llll}\text { C } & 1.541149 & 2.287191 & -0.336565 \\ \text { C } & 1.986063 & 3.713938 & -0.322881\end{array}$ C $1.9860633 .713938-0.322881$ C $-0.655416 \quad 2.804900-0.352990$ C -0.6554162 .8049000 .352990$ $\begin{array}{llll}\text { C } 0.047071 & -2.160213 & -0.074236\end{array}$ H $-0.220925-2.573104-1.060034$ $\begin{array}{llll}C-0.891390 & -2.691480 & 0.98674\end{array}$ $\begin{array}{llll}\text { C }-0.888805 & -4.230500 & 0.942363\end{array}$ H $-0.594933-2.354557 \quad 1.986844$ H $-1.918391-2.3239520 .831174$ H $0.126210 \quad-4.627439 \quad 1.098628$ H $-1.536351-4.6295281 .738108$ н $-1.256534-4.610636-0.023782$ H $1.099695-2.410003 \quad 0.134825$ C $-1.442271-0.247005-1.442605$ C $-0.233017-0.375739-2.180919$ H $-1.979487 \quad 0.699324-1.389041$ H $-2.045779-1.143633-1.291919$ H $-2.045779-1.143633-1.291919$ $\begin{array}{llll}\text { C } & 0.416491 & 0.653042 & -2.923158\end{array}$ H $0.052026-1.368008-2.54165$ $\begin{array}{lllll}\text { H } & 4.660415 & 0.745711 & -0.332766\end{array}$ $\begin{array}{llll}\mathrm{N} 1.042218 & 1.436336 & -3.526951\end{array}$ H $4.265726 \quad 2.442501 \quad 0.054755$ H $4.1583431 .882420-1.621809$ H $2.628422 \quad 3.922212 \quad 0.548308$ H $2.576625 \quad 3.925843 \quad-1.228455$ $\begin{array}{lllll}\text { H } & 1.125507 & 4.391961 & -0.280157\end{array}$ C $-0.555083 \quad 3.063565 \quad 1.742456$ C $-1.5490793 .844796 \quad 2.333098$ $\begin{array}{llll}\text { C }-2.587550 & 4.383168 & 1.585415\end{array}$ $\begin{array}{lllll}\text { C }-2.635232 & 4.162311 & 0.213462\end{array}$ C $-1.677844 \quad 3.373899-0.430378$ $\begin{array}{llll}C & -1.677844 & 3.373899 & -0.430378\end{array}$ $\begin{array}{llll}C & -1.704757 & 3.247201 & -1.942638\end{array}$ $\begin{array}{llll}C & -3.112349 & 3.004189 & -2.50140\end{array}$ $\begin{array}{llll}c & -1.076483 & 4.480751-2.612924\end{array}$ $\mathrm{H}-1.0727442 .39532-2.221868$ H $-3.6292312 .188100-1.974771$ $\mathrm{H}-3.0517312 .749014 \quad-3.57023$ H $-3.7379493 .905806-2.415137$ H $-0.031488 \quad 4.620588-2.302237$ н $-1.088387 \quad 4.363715-3.707564$ H $-1.638468 \quad 5.391427 \quad-2.349942$ H $-3.432430 \quad 4.617411-0.374018$ H $-3.355356 \quad 4.987445 \quad 2.069131$ H $-1.499764 \quad 4.024465 \quad 3.407938$ C $3.128192-1.523714 \quad 1.458860$ $\begin{array}{llll}\text { C } 4.055404 & -2.545550 & 1.734777\end{array}$ $\begin{array}{llll}C & 4.836108 & -3.5455506 & 0.7347201\end{array}$ $\begin{array}{llll}\text { C } 4.836108 & -3.102106 & 0.727201\end{array}$ $\begin{array}{llll}\text { C } 4.682018 & -2.658585 & -0.588310 \\ \text { C } 3.788489 & -1.632775 & -0.915030\end{array}$ $\begin{array}{llll}C & 3.788489 & -1.632775 & -0.915030\end{array}$ C $3.589218-1.254803-2.36979$ c $4.926570-0.887449-3.095223$ C $2.886789-2.391283-3.129563$ H $2.940681-0.370034-2.422547$ H $5.480386-0.101800-2.557463$ H $5.582007-1.769691-3.181752$ H $4.704346-0.527355-4.113292$ н $1.962826-2.700529-2.618264$ H $2.637739-2.070235-4.153334$ H $3.537821-3.278475-3.195266$ H $5.268157-3.127010-1.382304$ H $5.546796-3.899896 \quad 0.956102$ 


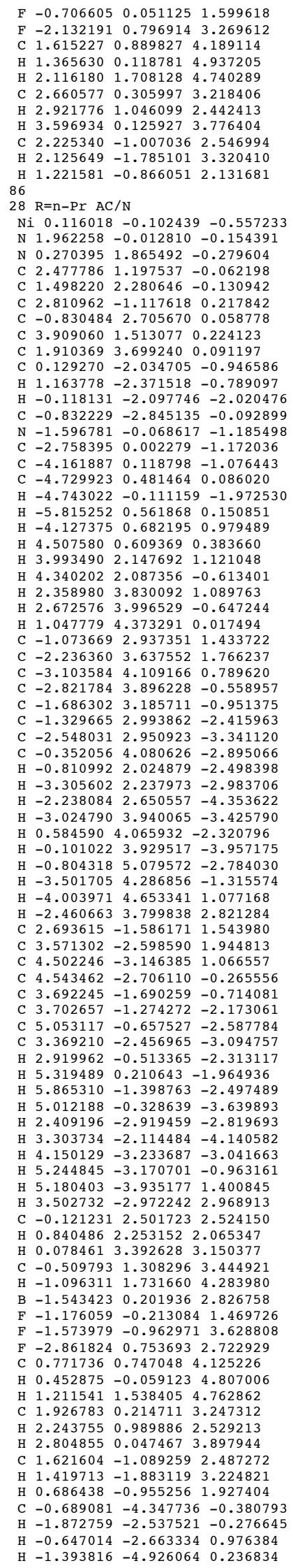

H $-0.896034-4.573248-1.438973$

H $0.329450-4.697819-0.152077$ 